\title{
Using Novel Data Types for Big Data Research in Epilepsy: Patient Records, Clinic Letters and Genetic Mutation
}

\author{
Lacey, Arron S.
}

How to cite:

Lacey, Arron S. (2019) Using Novel Data Types for Big Data Research in Epilepsy: Patient Records, Clinic Letters and Genetic Mutation. Doctoral thesis, Swansea University.

http://cronfa.swan.ac.uk/Record/cronfa48905

Use policy:

This item is brought to you by Swansea University. Any person downloading material is agreeing to abide by the terms of the repository licence: copies of full text items may be used or reproduced in any format or medium, without prior permission for personal research or study, educational or non-commercial purposes only. The copyright for any work remains with the original author unless otherwise specified. The full-text must not be sold in any format or medium without the formal permission of the copyright holder. Permission for multiple reproductions should be obtained from the original author.

Authors are personally responsible for adhering to copyright and publisher restrictions when uploading content to the repository.

Please link to the metadata record in the Swansea University repository, Cronfa (link given in the citation reference above.)

http://www.swansea.ac.uk/library/researchsupport/ris-support/ 
Swansea University

Prifysgol Abertawe

\section{Using Novel Data Types for Big Data Research in Epilepsy: Patient Records, Clinic Letters and Genetic Mutation}

Submitted to Swansea University in fulfilment of the requirements for the Degree of Doctor of Philosophy

\section{Arron Lacey \\ BSc MSc MRes}

Data Science

Swansea Neurology Research Group Swansea University Medical

School

Swansea University

February 2019 


\section{Declaration}

I, Arron Lacey, confirm this thesis has not been submitted towards a previous degree or other qualification, and is intended for submission of a Doctor of Philosophy, awarded by Swansea University.

Signed: $\quad$ (candidate) Date:

I, Arron Lacey, confirm that all of the work presented in this thesis is my own unless otherwise indicated. I have provided footnotes in the text where I have received assistance and have indicated permissions for presenting work which is not my own.

Signed: $\quad$ (candidate) Date:

I hereby give consent for my thesis, if accepted, to be available for photocopying and for inter-library loans after expiry of a bar on access approved by the Swansea University.

Signed: $\quad$ (candidate) Date: 


\section{Abstract}

Introduction: The aims of this thesis was to explore novel data types in healthcare that could enhance epidemiology studies in epilepsy and to develop novel methods of analysing routinely collected linked healthcare data, unstructured free text in hospital clinic letters and genetic variation.

Method: The SAIL Databank was used to source linked healthcare data for people with epilepsy across Wales to study the effects of epilepsy and social deprivation, coding of epilepsy in GP records and the educational attainment of children born to mothers with epilepsy. Hospital clinic letters from Morriston Hospital in Swansea were analysed using Natural Language Processing techniques to extract rich clinic data not typically recorded as part of routinely collected data. An automated pipeline was developed to predict the pathogenicity of Single Nucleotide Polymorphisms to prioritize potential disease-causing genetic variation in epilepsy for further in-vitro analysis.

Results: Incidence and prevalence of epilepsy was found to be strongly correlated with increased social deprivation, however a 10 year retrospective follow-up study found that there was no increase in deprivation following a diagnosis of epilepsy, pointing to deprivation contributing to social causation of epilepsy rather than epilepsy causing social drift. An algorithm was developed to accurately source epilepsy patients from GP records. Sodium Valproate was found to reduce educational attainment in 7 year olds by $12 \%$. A Natural Language Processing pipeline was developed to extract rich epilepsy information from clinic letters. A pipeline was created to predict pathogencity of epilepsy SNPs that performed better than commonly used software.

Conclusion: This thesis presents novel studies in epilepsy using population level healthcare data, unstructured clinic letters and genetic variation. New methods were developed that have the potential to be applied to other disease areas and used to link different data types into routinely collected healthcare records to enhance further research. 


\section{Contents}

1 Introduction $\quad 19$

1.1 Summary of Thesis Themes . . . . . . . . . . . . . . . . . 19

1.2 Epilepsy ......................... 20

1.2.1 Epilepsy types . . . . . . . . . . . . . . . 20

1.2.2 Generalized seizures . . . . . . . . . . . . . . . . 21

1.2.3 Focal seizures . . . . . . . . . . . . . . . . . . . . 22

1.2.4 Causes of epilepsy . . . . . . . . . . . . . . . . . 23

1.2.5 Genetics of epilepsy . . . . . . . . . . . . . . . 24

1.2.6 Epidemiology of epilepsy . . . . . . . . . . . . . 25

1.2.7 Anti-epileptic drugs . . . . . . . . . . . . . . . . . 26

1.2.8 Burden and impact of epilepsy . . . . . . . . . . . . . . 28

1.3 Big data and patient records as a resource for research . . . . . . . . 29

1.3.1 Electronic Healthcare Records . . . . . . . . . . . . . . . . . . 30

1.3.2 The SAIL Databank . . . . . . . . . . . . . . . . . . 32

1.3.3 Anonymous patient records . . . . . . . . . . . . . . 33

1.3.4 SAIL studies . . . . . . . . . . . . . . . 34

1.4 Natural Language Processing: Using clinic letters as a data source for

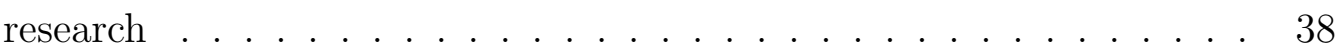

1.4.1 Part of Speech Tagging . . . . . . . . . . . . . . . . . 39

1.4.2 Shallow parsing . . . . . . . . . . . . . . . 41

1.4.3 Named Entity Recognition . . . . . . . . . . . . . . . . . . . 42

1.4.4 NLP tools and software . . . . . . . . . . . . . . . . . . . 44

1.4.5 Validating NLP algorithms . . . . . . . . . . . . . . . 45

1.4.6 NLP clinical information applications . . . . . . . . . . . . 46

1.4.7 Genetic Mutation . . . . . . . . . . . . . . . . . . . . . 48

1.5 Pathogenicity of SNPs . . . . . . . . . . . . . . . . . . 49

1.6 Whole genome sequencing and the need for SNP prediction paradigms 50

1.7 Common features used in prediction of pathogenicity . . . . . . . . . 52

1.7.1 Machine learning classification for SNP pathogenicity . . . . . 54 
1.7.2 SNP datasets . . . . . . . . . . . . . . . . 55

1.7.3 Bioinformatics software and annotation programs to obtain

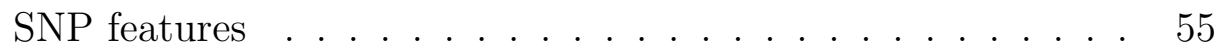

1.8 Chapter Summary . . . . . . . . . . . . . . . . . . . 56

1.9 Summary of aims and objectives . . . . . . . . . . . . 57

2 Methods $\quad 58$

2.1 The SAIL Databank . . . . . . . . . . . . . . . . 58

2.1.1 Ethics and Governance . . . . . . . . . . . . . . . . . 59

2.1.2 Assessing the burden of disease using the SAIL Databank . . . 59

2.1.3 Forming Research Questions . . . . . . . . . . . . . 60

2.2 SAIL Datasets . . . . . . . . . . . . . . . . . . . 61

2.2 .1 GP dataset . . . . . . . . . . . . . . . . . . 61

2.2 .2 Secondary Care dataset $\ldots \ldots \ldots \ldots$. . . . . . . . . 62

2.2 .3 Welsh Demographic Service . . . . . . . . . . . . . . 62

2.2 .4 ONS deaths . . . . . . . . . . . . . . . . . . . 64

2.3 Data Linkage . . . . . . . . . . . . . . . . . . . . . . . . . 64

2.3.1 Structured Query Language . . . . . . . . . . . . . 64

2.3.2 Quality checking routinely collected data . . . . . . . . 65

2.3 .3 Statistical Analysis . . . . . . . . . . . . . . . . 67

2.4 Natural Language Processing . . . . . . . . . . . . . . . . 68

2.4 .1 Software . . . . . . . . . . . . . . . . 68

2.4.2 Part of Speech tagging . . . . . . . . . . . . . . . 69

2.4.3 Gazetteers . . . . . . . . . . . . . . . . . . . . 70

2.4.4 Context Algorithm . . . . . . . . . . . . . . . . . . . 71

2.4 .5 JAPE rules . . . . . . . . . . . . . . . . . . . 72

2.5 Predicting functional impact of Single Nucleotide Polymorphisms . 74

2.5.1 Pipeline to determine the effect of SNPs . . . . . . . . 74

2.5.2 Data sources . . . . . . . . . . . . . . . . . . . . 74

2.5.3 Obtaining Protein Features _. . . . . . . . . . . 75

2.5.4 Predicting SNP Impact Using Machine Learning . . . . . . . . 81

2.5.5 Training and testing . . . . . . . . . . . . . 82

2.5.6 Receiver Operator Curves . . . . . . . . . . . . . . . . 82

2.6 Chapter Summary . . . . . . . . . . . . . . . . . . . . 83

3 Results: Analysing Routinely Collected Healthcare Records for Epilepsy Research 85

3.1 Prevalence, Incidence and the Social Deprivation Profile of Epilepsy in Wales . . . . . . . . . . . . . . . . . . . . 85 
3.1.1 Defining Epilepsy in the SAIL Databank . . . . . . . . . . . . 86

3.1.2 Social deprivation . . . . . . . . . . . . . . . . . . 91

3.1.3 Follow up cohort . . . . . . . . . . . . . . . . 97

3.2 Validating epilepsy status from electronic healthcare records . . . . . 100

3.2.1 Study population . . . . . . . . . . . . . . 100

3.2.2 Algorithm validation . . . . . . . . . . . . . . . 100

3.3 Educational attainment of children born to mothers with epilepsy . . 104

3.3.1 Cohort selection . . . . . . . . . . . . . . . . . . 104

3.3.2 Education dataset . . . . . . . . . . . . . . . 106

3.3 .3 Results . . . . . . . . . . . . . . . . . . 108

3.4 Chapter summary . . . . . . . . . . . . . . . 111

4 Using Natural Language Processing techniques to extract clinical information from unstructured text 112

4.1 Clinic letters . . . . . . . . . . . . . . . . . . . . 112

4.2 A rule based NLP approach to extract epilepsy information from clinic

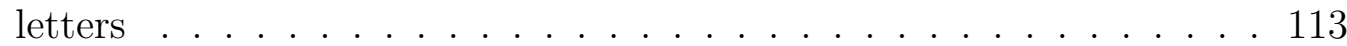

4.2.1 The General Architecture for Text Engineering . . . . . . . . 113

4.2 .2 Defining rules . . . . . . . . . . . . . . . 115

4.2.3 Clinic date and date of birth . . . . . . . . . . . . . 116

4.2.4 Epilepsy diagnosis, epilepsy type and seizure type . . . . . . . 116

4.2.5 Seizure frequency . . . . . . . . . . . . . . 121

4.2 .6 Medication . . . . . . . . . . . . . . . . 123

4.2.7 Investigations - CT, MRI and EEG scans . . . . . . . . . . . . 125

4.2.8 Validation of Algorithm . . . . . . . . . . . . . . . . 128

4.3 Chapter Summary . . . . . . . . . . . . . . . 132

5 Predicting functional impact of Single Nucleotide Polymorphisms 133

5.1 Features . . . . . . . . . . . . . . . . . . . 133

5.1.1 Variant Effect Predictor . . . . . . . . . . . . . . . 134

5.1 .2 CTD Descriptors .................... 136

5.1.3 Secondary structure prediction . . . . . . . . . . . . . 138

5.1 .4 Results. . . . . . . . . . . . . . . . . . 141

5.1.5 Comparison of Random Forest to other classifiers . . . . . . . 145

5.2 Functional Analysis of SNPs associated with Epilepsy . . . . . . . . . 147

5.3 Summary of Results . . . . . . . . . . . . . . . . 150

6 Discussion 153

6.1 SAIL studies . . . . . . . . . . . . . . . . . 153

6.1.1 Social deprivation and epilepsy . . . . . . . . . . . 154 
6.1.2 Validation of epilepsy algorithm using a gold standard dataset 156

6.1.3 Educational attainment of children born to mother's with epilepsy158

6.2 Natural Language Processing of epilepsy clinic letters . . . . . . . . . 160

6.3 Predicting pathogenicity of SNPs for large datasets . . . . . . . . . . 164

6.3.1 Use of existing predictors as features . . . . . . . . . . 166

6.3.2 Use of physiochemical properties and predicted secondary structure . . . . . . . . . . . . . . . . . . 166

6.4 Future Work . . . . . . . . . . . . . . . . . . . 167

6.5 Conclusions . . . . . . . . . . . . . . . . . . . . . . 168 


\section{List of Figures}

1.1 Incidence of epilepsy from a study in Iceland showing incidence per 100,000 stratified by age and sex. . . . . . . . . . . 26

1.2 Prescribing trends of first line AEDs in Wales between 2000-2010. Newer drugs such as Lamotrigine have been adopted as per the SANAD study guidelines, where a decline in valproate prescriptions to women of child bearing age is also observed $[1] \ldots \ldots \ldots$. . . . . . 27

1.3 The core SAIL datasets. Each dataset can be linked anonymously via an encrypted NHS number . . . . . . . . . . . . . 33

1.4 Decrease in whole genome sequencing since the Human Genome Project when compared to the expected rate of decrease following Moore's Law 51

1.5 The BLOSUM 62 matrix where higher scores indicate higher frequency of substitution. Each amino acid substitution is scored in accordance to it's frequency, where lower frequency substitutions are said to be conserved and potentially selected against by natural selection. . . . . 52

1.6 A multiple sequence alignment of transmembrane proteins from different species. Conserved regions are in red where alignment of different proteins shows no difference in amino acids across all proteins in this position. Conserved regions are therefore hypothesized not to tolerate geneitc variation and are deemed hotspots for pathogenic mutations. . . . . . . . . . . . . . . . 53

2.1 SAIL Databank split file procedure. Data is split at source into identifiable data and clinic data. The identifiable data sent to The NHS Wales Information Service where each NHS number is encrypted before being sent to the SAIL Databank. The clinic data is sent directly to the SAIL Databank, and is joined to the encrypted identifiable data by an internal system ID that is present in both datasets. . . . . . . . 59

2.2 Flow chart of how the WIMD score is calculated from 8 different domains. Taken from http://webarchive.nationalarchives.gov . uk/20150505155421/http://gov . wales/docs/statistics/2011/ 110831wimd11summaryen.pdf . . . . . . . . . . . . . . . . 
2.4 A example clinic letter. The letter contains real patient data, but all identifiable information has been anonymized . . . . . . . . . . . . . 68

2.7 A flow chart of the pipeline. Purple nodes are databases and green nodes are processes. The user can specify SNPs in chromosomal format as input to the pipeline. The end result is the SNP data with protein level data that includes indexes generated by downstream programs and database annotation. . . . . . . . . . . . . . . . . . 76

2.10 PSIPRED algorithm: multiple sequence alignments of known protein structures are built up from an input sequence. Position specific scoring matrix is used to train a neural network to predict the secondary structure of novel proteins. . . . . . . . . . . . . . .

2.11 PSIPRED output comparing the predicted secondary structure of a wild type GLRA2 sequence with the same sequence having a clinically benign SNP at position 355 of the sequence. The secondary structure prediction is normalised between coil, helix and sheet, where the absolute difference between the wild type and SNP are calculated in the three rightmost columns. The red line indicates the SNP, where other lines are neighbouring amino acids and predictions. It can be seen that while the predicted secondary structure doesn't change, the amino acids closer to the SNP have a larger change in the raw score than those further out from the SNP. . . . . . . . . . . . . . . .

2.13 ROC curve comparing the classifier from this thesis (black) to scores from other classifiers when predicting disease/benign status on the humvar test set . . . . . . . . . . . . . . . . . . .

3.1 Table 1 of 2 defining QOF codes for recording information on epilepsy in patient records. Table taken from https://www.epilepsy.org.u k/sites/epilepsy/files/primary-care-resource/A18-Tool.pdf .

3.2 Table 2 of 2 defining QOF codes for recording information on epilepsy in patient records. Table taken from https://www.epilepsy.org.uk/ sites/epilepsy/files/primary-care-resource/A18-Tool.pdf .

3.3 Visual explanation of the algorithm used to capture epilepsy diagnoses. All AED prescriptions are first found using GP records (D1), in which AED prescriptions pairs within 6 months after the initial prescription are classed as a repeat AED prescription (D2). Epilepsy diagnosis codes appearing in GP records 12 months either side of the first prescription of each of the AED pairs are queried, and where there is a match a person is classified by the algorithm as having an epilepsy diagnosis at the time of the first AED in the pair. . . . . . . . . . . . . . . . 
3.6 Flow chart of cohort selection. GP records were used to identify people with epilepsy (and therefore those that did not have epilepsy). The Welsh Demographic Service dataset was then used to sample age bands, sex and WIMD deciles on the first of January in each study year. For every unique combination of covariates, incidence and prevalence was calculated. . . . . . . . . . . . . . . . . . . .

3.7 Plots of (A) epilepsy prevalence and (B) epilepsy incidence by WIMD (deprivation) decile. Error bars indicate 95\% confidence intervals. Figure taken from $[4] \ldots \ldots$. . . . . . . . . . . . .

3.8 Maps of Wales showing each LSOA (areas with population of around $1,500)$; Yellow areas represent with low data coverage ( $5 \%$ of the population) and are not shown. (A) Deprivation measured by WIMD decile, (B) epilepsy prevalence, and (C) epilepsy incidence. Enlarged areas represent the densely populated areas of the cities of Swansea, Cardiff, and Newport (left to right). Figure taken from [4] . . . . . .

3.9 Changes in WIMD decile over 10 years for with incident epilepsy diagnosed between January 1, 2000 and December 31, 2002. Figure taken from $[4] \ldots \ldots \ldots \ldots \ldots \ldots$

3.10 The accuracy of algorithms A,B and $\mathrm{C}$ in being able to determine epilepsy status from GP records. Table taken from [5] . . . . . . . . . 102

3.11 Flow chart of cohort ascertainment. 4 datasets were queried: General Practice, ONS Births, Welsh Demographic Service and Welsh Education Dataset. . . . . . . . . . . . . . . . .

3.12 Descriptive statistics of the study cohort. The control group comprised of a 1:4 match on maternal age, gestational age and Welsh Index of Multiple Deprivation (WIMD) quintile. WIMD quintiles are a measure of deprivation (see method) with quintile 1 being the most deprived and quintile 5 being the least deprived. sd=standard deviation. * ${ }^{*}$-values are for comparisons between each group within the "Mothers with Epilepsy" group with the control group. Table taken from [3] . . . . .

3.13 Key Stage 1 results stratified by subject and study groups. Each group was compared to the matched control group. Significant differences in attainment (* p i $0.05, * * \mathrm{p} ; 0.005)$ between each group and the matched control are shown. The p-values have been Bonferroni corrected for multiple testing (see Methods section). The All Wales group is shown as a regional comparator only and not used to test for significance. Figure taken from $[3]$. . . . . . . . . . . . . . . . . . . 110 
4.1 Overview of the GATE pipeline and the various components used to generate annotations . . . . . . . . . . . . . . . . . . . 114

4.6 A Lookup for the phrase "possible complex partial seizures". The "Certainty" features was added through development of custom gazetteers and JAPE rules. The rest of the features come as default from the BIO-Yodie plugin in GATE, and the "Negation" feature was produced by modifying the Context plugin in GATE to add more stop words. . . . . . . . . . . . . . . . . . . 12

5.1 Pipeline of SNP data collection. The data is used to train a classifier that can be used to predict disease/benign status of a SNP. . . . . . . 134

5.7 Comparison of two sample PSIPRED output files, where the left shows predictions for the wild type protein and the right shows the same sequence with the SNP is inserted. Lines are colour coded by increasing difference in prediction probabilities between the wild type and SNP sequence, where red depicts the largest difference and yellow the smallest.140

5.9 Feature importance ranked by the mean decrease in accuracy when each feature is excluded from the Random Forest model . . . . . . . . 144

5.10 Feature importance ranked by the mean decrease in accuracy when each feature is excluded from the Random Forest model . . . . . . . . 145

5.11 ROC curve comparing the classifier from this thesis (black) to scores from other classifiers when predicting disease/benign status on the humvar test set . . . . . . . . . . . . . . . . . . . 146

5.12 Specificity plot of each algorithm when sensitivity is set to $95 \%$. Only algorithms that could achieve 95\% sensitivity are presented . . . . . . 147

5.13 ROC curve comparing the classifier from this thesis (black) to rankscores from other classifiers when predicting disease/benign status for SNPs found in genes associated with epilepsy . . . . . . . . . . . . 149

5.14 Specificity plot of each algorithm when sensitivity is set to $95 \%$. Only algorithms that could achieve 95\% sensitivity are presented . . . . . . 150 


\section{List of Tables}

1.1 Generalized seizures recognized by the ILAE. . . . . . . . . . . . . . . 22

1.2 Focal seizures recognized by the ILAE . . . . . . . . . . . . . . 23

1.3 A list of ion channel domains and proteins and how mutations correlate to epilepsy phenotypes. . . . . . . . . . . . . . . . . . 24

1.4 A table of PENN treebank POS tags. https://www.ling.upenn. edu /courses/Fall_2003/ling001/penn_treebank_pos.html . . . . . . 40

1.5 The chunk types defined as part of the CONLL-2000 shared task [2] . 42

1.6 A list of common NLP software . . . . . . . . . . . . . . . . . . . . 44

1.7 Commonly used SNP prediction programs that utilize machine learning 54

2.1 ANNIE POS tags and their descriptions . . . . . . . . . . . . . 70

2.2 UMLS representation of a subset of epilepsy terms. The CUI (Concept Unique Identifier) is a code assigned to biomedical concepts. The source column represents the original coding system the term exists in, and the SCUI column is the source code used within a particular system. For example "Epilepsy" exists in both ICD10 and READ coding systems, but map to the same CUI in UMLS despite having unrelated SCUIs. . . . . . . . . . . . . . . . . . . . . 71

2.3 List of JAPE operators than can be applied to any annotation . . . . 74

2.4 List of popular SNP prediction software . . . . . . . . . . . . . . . 79

2.5 Machine learning algorithms used in SNP prediction in chapter 5 . . . 82

3.1 SAILWGPV.EVENT_ALF_E is a table in the SAIL Databank that stores GP patient records. . . . . . . . . . . . . . . . 86

3.2 The SAILWDSDV.AR_PERS table in the SAIL Databank holds individuals address, provided as the address when registering with a GP. Each address is also assigned to a Lower Super Output Area (LSOA) which is a geographical area comprising of around 1500 individuals. . 91

3.3 The SAILREFRV.WIMD2008_OVERALL_INDEX table in the SAIL Databank contains a link between an LSOA code and various Welsh Index of Multiple Deprivation measures. . . . . . . . . . . . . . . . . 91 
3.4 Study population characteristics in 2010 as compared to the Welsh population (measured by the 2011 WIMD data). Table taken from [4] 93

3.5 Breakdown of epilepsy prevalence and epilepsy incidence by WIMD decile. Table taken from $[4]$. . . . . . . . . . . . . . . . . . . . . 94

3.6 Variable Adjusted epilepsy prevalence odds ratio Adjusted epilepsy incidence rate ratio The odds and incidence rate ratios for deprivation (second row of the table) are given per WIMD decile when compared to the population in decile 1 , for example, the odds ratio of epilepsy prevalence in WIMD decile $3=0.922 \times 0.94$ when compared to the population in decile 1 . Table taken from $[4]$. . . . . . . . . . . . . 97

3.7 Summary of follow up statistics for 10 year follow up cohort. Table taken from $[4]$. . . . . . . . . . . . . . . . . . . . . . . . . 98

3.8 SAILCHDV.CHILD is a table in the SAIL Databank that contains birth records and relates each child's NHS number to their mother. . 105

3.9 SAILDCELV.PRE16_KS1 is a table in the SAIL Databank contains all-Wales education data between 2003-2008 for Key Stage 1. Three subjects (Maths,Science,Engish/Welsh) as well as a Core Subject Indicator are provided to indicate the level of attainment per child . . 107

4.1 Definitions of each category intended to be extracted . . . . . . . . 115

4.2 A gazetteer of terms used to determine 5 levels of certainty attached to an epilepsy diagnosis. A confirmed diagnosis must have a value of 4 or 5.119

4.3 A sample of phrases used to define explicit time periods. . . . . . . . 123

4.4 A sample of UMLS terms and the important information within each. UMLS terms such as these shown are difficult to map directly to text within clinical texts, where the terms of interest from within each UMLS term are much easier to map. . . . . . . . . . . . . . . . . 127

4.5 A list of custom terms used to indicate a possible EEG finding . . . . 128

4.6 Precision, recall and F1-score are calculated across 9 epilepsy specific categories as well as clinic date and date of birth. Two approaches have been considered - the first measures the algorithm's accuracy for every mention $(\mathrm{N}=1925)$ across all categories as identified in the manual review by the clinician, and the second approach aggregates results from multiple mentions per category, per letter i.e. if there are multiple true mentions regarding confirmation of epilepsy in a single letter, we assign a single true positive providing the algorithm picks up at least one of these mentions, with the same logic used to determine false positives, true negatives and false negatives. . . . . . . . . . . . 131

5.1 Features obtained from VEP . . . . . . . . . . . . . 135 
5.2 Amino acid attributes with three group classification. Each classification is given by a unique set of amino acids. Table reproduced with permission from https://cran.r-project.org/web/packages $/$ protr/vignettes/protr.html . . . . . . . . . . . 136

5.35 fold cross-validation of the 6 classifiers. . . . . . . . . . . . . . . 143

5.4 Frequency table of genes associated with epilepsy sourced from Clinvar 148

5.5 Confusion matrix for Random Forest classifier showing the number of observed vs predicted classifications in 301 SNPs found in genes associated with epilepsy. . . . . . . . . . . . . . . . 150

5.6 Comparison of true pathogenic SNPs and predicted pathogenic SNPs in each gene . . . . . . . . . . . . . . . . . . 151

6.1 Read codes used to signify a diagnosis of epilepsy . . . . . . . . . 170 


\section{Acronyms}

1000g 1000 Genomes

AED Anti-Epileptic Drug

ALF Anonymous Linking Field

Bash Bourne Again SHell

BLAST basic local alignment search tool

BLOSUM BLOcks SUbstitution Matrix

CADD Combined Annotation Dependent Depletion

CBZ carbamazepine

CI confidence interval

CIPHER Centre for the Improvement of Population Health through E-records Research

Clinvar Clinical Variation database

CSV Comma Separated Variable

CUI Concept Unique Identifier

DALY Disability Adjusted Life Year

dbSNP Single Nucleotide Polymorphism atabase

DNA deoxyribonucleic acid

DSSP Dictionary of Secondary Structure in Proteins

EEG electroencephalogram

epi4k Epilepsy 4000 genomes project 
ExAc Exome Aggregation Consortium

GATE General Architecture for Text Engineering

gnomAD Genome Aggregation Database

GUI Graphical User Interface

GWAS Genome Wide Association study

HGMD Human Gene Mutation Database

HPC High Performance Computer

ICD International Classification of Diseases

IGRP Information Governance Review Panel

ILAE International League Against Epilepsy

indel insertions/deletion

IQ Intelligence Quotient

JAPE Java Annotations Pattern Engine

LEV levetiracetam

LTG lamotrigine

LSOA Lower Super Output Area

MAF Minor Allele Frequency

MESH Medical Subject Headings

MRI Magnetic Resonance Image

MRCONSO UMLS Concept file

MRREL UMLS Relationship file

NGS Next Generation Sequencing

NHS National Health Service

NWIS NHS Wales Informatics Service

OR odds ratio

PEDW Patient Episode Database for Wales 
PHT phenytoin

POS Part Of Speech

PSIBLAST Position Specific Iterated BLAST

PSIPRED PSI Blast Prediction for secondary structure

R $\mathrm{R}$ programming language

READ READ clinical coding system

SAIL Secure Anonymised Information Data Linkage

SD standard deviation

SIFT Sorting Intolerant From Tolerant

SNOMED SNOMED clinical coding system

SNP Single Nucleotide Polymorphism

SQL Standard Query Language

SUDEP Sudden Unexplained Death in Epilepsy

SVM Support Vector Machine

TPM topiramate

UCSC University of California, Santa Cruz

UK United Kingdom

UMLS Unified Medical Language System

Uniprot the Universal Protein Resource

UNIX UNIX operating system

VCF variant call format

VEP Variant Effect Predictor

VPA sodium valproate

WES Whole Exome Sequencing

WERN Wales Epilepsy Research Network

WGS whole genome sequencing 
WIMD Welsh index of multiple deprivation 


\section{Acknowledgements}

I would like to thank my supervisors Professor Mark Rees and Dr Seokyung Chung for providing me with their guidance and support. Their experience was vital in helping shape this thesis and it would have not been completed without their help. I would also like to thank Dr Owen Pickrell for providing me with invaluable experience and expertise that was essential towards carrying out the studies in this thesis. I would also like to thank Beata Fonferko-Shadrach for her keen eye for detail and help with the analysis of clinic letters and GP records.

I would like to thank the Swansea Neurology Research Group for their support: Dr Rhys Thomas, Dr Cathy White and Professor Mike Kerr, Dr Anna Derrick, Adam Higgins and Sarah Dawes. I would also like to thank my colleagues at the SAIL Databank for both their professional and personal support (cakes), particularly Ashley Akbari and Daniel Thayer for line managing me, Simon Thompson for his technical support and Professor Ronan Lyons and Professor David Ford for the opportunities they have given me.

Finally I would like to thank my family, especially my wife Sophie Lacey for her unending encouragement and understanding during completion of this thesis as well as my son Dylan for providing many welcome distractions from both study and sleep over the last 18 months.

This work has not previously been accepted in substance for any degree and is not being concurrently submitted in candidature for any degree.

\section{Papers related to Thesis}

- Lacey, A.S., Pickrell, W.O., Thomas, R.H., Kerr, M.P., White, C.P. and Rees, M.I., 2018. Educational attainment of children born to mothers with epilepsy. J Neurol Neurosurg Psychiatry, pp.jnnp-2017. [3]

- Pickrell, W.O., Lacey, A.S., Bodger, O.G., Demmler, J.C., Thomas, R.H., Lyons, R.A., Smith, P.E., Rees, M.I. and Kerr, M.P., 2015. Epilepsy and deprivation, a data linkage study. Epilepsia, 56(4), pp.585-591. [4]

- Fonferko-Shadrach, B., Lacey, A.S., White, C.P., Powell, H.R., Sawhney, I.M., Lyons, R.A., Smith, P.E., Kerr, M.P., Rees, M.I. and Pickrell, W.O., 2017. Validating epilepsy diagnoses in routinely collected data. Seizure-European Journal of Epilepsy, 52, pp.195-198.[5] 


\section{Chapter 1}

\section{Introduction}

\subsection{Summary of Thesis Themes}

This thesis documents research in epilepsy across 3 themes: epidemiology and big data, natural language processing of clinic letters and predicting pathogenicity of single nucleotide polymorphisms. The main aim was to describe how different methods and data types across these themes can be brought together to enhance the opportunity for epilepsy based research. This chapter introduces relevant studies related to the three themes of research to support the motivations for this thesis, and chapter two describes a comprehensive overview of the methods used to carry the work in this thesis.

Chapter 3 documents the results for 3 longitudinal epidemiological studies in epilepsy using the SAIL Databank. The SAIL Databank is a research platform for conducting population level healthcare studies in Wales and specialises in anonymous linked "Big Data" across various healthcare services across Wales. The three studies in chapter 3 explore the effects of epilepsy on social deprivation, a validation of GP recorded epilepsy diagnoses and the effects of exposure to antiepileptic drugs in the womb and the impact it has on educational attainment in 7 year old children.

Chapter 4 aims to explore how data in unstructured clinic letters can be included in epidemiology studies by using Natural Language Processing techniques. Only a proportion of unstructured data such as that in clinic letters, discharge reports or radiology and examination reports get entered into structure databases and audited for research purposes, with many rich patient data often missing and not available for research. Chapter 4 presents a study using NLP techniques to extract rich patient information from 200 clinic letters from the Morriston hospital epilepsy clinic. 
Chapter 5 explores various techniques to predict pathogencity of a particular type of genetic mutation called singe nucleotide polymorphisms, or SNPs. There are many SNPs that have been documented as the cause of various types of epilepsy, but with over 3 million variants in a person's genome it is difficult to predict the impact of these mutations in terms of likelihood of developing a disease. Bioinformatics pipelines aim to reduce the search space within the human genome to focus on a very small set of variants for further study. Part of these pipelines involves functional analysis and there are many programs that specialize in predicting pathogenicity of SNPs, in which the accuracy of these programs can differ in different disease areas. Chapter 5 aims to incorporate the knowledge from existing systems to build a pipeline that accurately predict the pathogenicity of epilepsy SNPs.

\section{$1.2 \quad$ Epilepsy}

Epilepsy is a disease characterized by unprovoked seizures that can be distinctly different from other types of seizures such as febrile seizures that occur mainly in children during a fever and dissociative seizures that occur for psychological reasons. It effects $1 \%$ of the population (600,000 individuals in the UK) [6] [7] and it has been estimated that over 50 million people worldwide have epilepsy [8]. The International League Against Epilepsy (ILAE) define epilepsy as any one of the following [9]:

1. Two unprovoked seizures occurring more than 24 hours apart

2. One unprovoked (or reflex) seizure and a probability of further seizures similar to the general recurrence risk (at least 60\%) after two unprovoked seizures, occurring over the next 10 years

3. A diagnosis of an epilepsy syndrome

Epileptic seizures are treated with anti-epileptic drugs (AEDs) in which patients may require a combination of AEDs to help control their seizures, and some may not respond to AEDs at all, known as refractory epilepsy.

\subsubsection{Epilepsy types}

There are various epilepsy types that can be defined in various ways:

- Type of seizure

- Age at which seizure began

- Causes of seizure 
- The part of the brain involved during a seizure

- Severity and duration of seizures

- EEG electroencephalogram patterns

- Brain imaging

- Mode of inheritance

- Other disorders in additions to seizures

- Patterns of seizures during the day (at day or night)

While there are many syndromes within epilepsy such as Juvenile Myclonic Epilepsy, Dravet Syndrome and Lennox-Gaut syndrome, epilepsy seizures are also used as a diagnostic tool in clinical practice and combined with various other factors seizure type will underpin an epilepsy syndrome. The use of seizures as a diagnostic tool is useful in terms of choosing AED treatment, where different seizure types have well defined AED regimes. Seizure categories in epilepsy are broadly defined by two types, that being generalized or focal seizures.

\subsubsection{Generalized seizures}

Generalized seizures originate rapidly from bilaterally distributed brain networks i.e. generalized seizures affect the entire brain [10]. The ILAE recognize generalised seizures as described in table 1.1. [11] 
Table 1.1: Generalized seizures recognized by the ILAE.

\begin{tabular}{|c|c|}
\hline Name & Description \\
\hline Tonic-clonic seizures & $\begin{array}{l}\text { Initial phase of stiffness (tonic) followed by jerking (clonic) and a } \\
\text { loss of consciousness. Gradual recovery with minute/hours of post } \\
\text { ictal confusion }\end{array}$ \\
\hline Clonic seizures & Similar to tonic clonic seizures without stiffness \\
\hline Typical absence seizures & $\begin{array}{l}\text { Sudden, brief (generally }<10 \mathrm{~s} \text { ) periods of loss of awareness } \\
\text { with behavioural arrest (staring episodes) with rapid recovery, } \\
\text { occasional eye movements and automatisms }\end{array}$ \\
\hline Atypical absence seizures & $\begin{array}{l}\text { Longer than typical absences and frequently associated with } \\
\text { myoclonic or atonic attacks. Start and finish more gradually, } \\
\text { focal features more prominent, and more retained awareness, than } \\
\text { typical absences }\end{array}$ \\
\hline Myoclonic absence seizures & $\begin{array}{l}\text { Very brief }(<1 \mathrm{sec}) \text { 'electric-shock' muscle contractions with } \\
\text { sudden onset and cessation. Single muscle to generalised jerking. } \\
\text { Consciousness generally not impaired }\end{array}$ \\
\hline Tonic seizures & $\begin{array}{l}\text { Sustained muscular contraction lasting }<1 \text { minutes with rapid } \\
\text { recovery }\end{array}$ \\
\hline Eyelid myoclonia & Quick upward jerk of the eyelids lasting around 3 seconds \\
\hline Myoclonus & $\begin{array}{l}\text { Spasmodic jerks or twitches in various muscles (positive) or brief } \\
\text { laspes in concentration (negative) }\end{array}$ \\
\hline Atonic seizures & $\begin{array}{l}\text { Sudden loss in muscle strength causing the patient to drop to floor. } \\
\text { Sometimes called "drop attacks" }\end{array}$ \\
\hline
\end{tabular}

\subsubsection{Focal seizures}

Focal seizures originate from one hemisphere in the brain in which some types may cause absences and loss of consciousness. Additionally some types of focal seizures can spread to the entire brain which are called secondary generalised seizures. The ILAE recognizes focal seizure types as described in table 1.2. 
Table 1.2: Focal seizures recognized by the ILAE

\begin{tabular}{ll}
\hline Name & Description \\
\hline Focal sensory seizures & Brief disturbance in taste, touch, smell or sight usually lasting no \\
more than 2 minutes & A seizure with localized motor activity. There may be spasm or \\
& clonus (jerking) of one muscle or a muscle group and this may \\
& remain localized or it may subsequently spread to adjacent muscles \\
& Frequently occurring during sleep. Brief, rapid onset and \\
Frontal lobe & cessation. Prominent motor features, sometimes with posturing \\
& and head version. Frequent bizarre automatisms / behaviours and \\
& vocalisation \\
& "Generally longer in duration than frontal lobe seizures. Variety of \\
& sensory disturbances including psychic (d'ej'a vu, jamaisvu, fear), \\
Temporal lobe & gustatory and olfactory hallucinations. Sensation of epigastric \\
& disturbance. Oro-facial automatisms (e.g. chewing, sucking) or \\
fidgety hand movements. Frequently altered awareness. Auditory \\
features with lateral temporal lobe involvement" \\
A rare type of seizure that involves a sudden burst of energy, \\
usually in the form of laughing or crying. \\
Entirely 1-sided, unilateral, clonic convulsions \\
Focal seizures evolving into generalized seizures, most often with \\
tonic-clonic convulsions. The partial seizures, which were once \\
limited to one hemisphere of the brain, progress to encompass the \\
entire brain bilaterally \\
Hemiclonic seizures \\
Secondarily generalized seizures \\
seizures
\end{tabular}

\subsubsection{Causes of epilepsy}

The causes of epilepsy can be broadly defined as either symptomatic where there is a physically identifiable change in structure of the brain, or genetic where there is no apparent change in structure of the brain and is therefore assumed to be caused by a genetic mutation inherited from a person's parents. Both types of epilepsy each account for around $50 \%$ of all epilepsies respectively. Symptomatic epilepsies are usually caused by injury to the brain through birth trauma, neurodegenerative diseases, brain neoplasms, cerebrovascular disease and brain malformations. For symptomatic epilepsy to be ruled out in the presence of epileptic seizures i.e. genetic epilepsy, there must be no evidence of structural changes in the brain to be detected. There exists a grey area over what constitutes symptomatic epilepsy or genetic epilepsy in that some conditions cause various deficiencies in the supply of glucose to the brain caused by a known genetic mutation, in which no structural changes are present in the brain, yet the patient is classified as having epilepsy. 


\subsubsection{Genetics of epilepsy}

For the $50 \%$ of epilepsies that are caused by genetic mutation, the incidence of genetic epilepsy passed on to first degree relatives have been shown to be up to 4 times that in the population than those that do not have a first degree relative with epilepsy [12]. Mutations found in a small amount of genes have been found to cause Idiopathic Generalized Epilepsy (IGE). Multiple family studies and twin studies have found that IGE has a common gene origin, but it is likely that some forms of epilepsy have multi-gene modes of inheritance [13] [14] [15]. There is evidence for different sets of genes producing different epilepsy syndromes such as Juvenile Myoclonic Epilepsy (JME) [16] [17]. Currently, mutations on the SCN1A voltage-gated sodium channel gene account for the largest amount of IGE syndromes [18] [19] with over 150 mutations attributed to infantile and childhood onset epilepsy. In general, seizure-related syndromes are accounted for by mutations across multiple genes that code for ion channel proteins, where examples of such proteins are given in table 1.3.

Table 1.3: A list of ion channel domains and proteins and how mutations correlate to epilepsy phenotypes.

\begin{tabular}{cccc}
\hline \multicolumn{4}{c}{ Channel Mutations in Epilepsy } \\
\hline Ion Channel & Gene & Phenotype & Inheritance \\
\hline Acetylcholine receptor & CHRNA2 & ADNFLE & Single Gene \\
& CHRNA4 & ADNFLE & Single Gene \\
& CHRNB2 & ADNFLE & Single Gene \\
Calcium & CACNA1A & CPS,GTCS & Single Gene \\
& CACNA1H & CAE,IGE & Complex \\
Chloride & CACNB4 & IGE & Complex \\
GABA receptor & CLCN2 & IGE & Single Gene \\
& GABRG2 & CAE/GEFS+/FS & Single Gene \\
Potassium & GABRA1 & JME,CAE & Single Gene \\
& KCNQ2 & BFNC1 & Single Gene \\
& KCNQ1 & BFNC1 & Single Gene \\
& KCND2 & mTLE2 & Single Gene \\
Sodium & SCN1A & GEFS/SMEI & Single Gene \\
& SCN2A & BFNIC & Single Gene \\
& SCN1B & GEFS+ & Single Gene \\
\hline
\end{tabular}

Despite some clear single gene relationships for various epilepsy syndromes there is also evidence for single gene overlap, and thus the relationship between known genes in epilepsy appears to be more complex[14]. Many factors determine development of the disease outside of an observed mutation such as mode of inheritance and 
gene expression, so presence of a mutation that is known to cause epilepsy in one person, may not cause epilepsy in another. Recruitment of families with an extensive history of a disease is the first challenge in furthering our understanding of the complex relationships of genes and disease. Sourcing cohorts of patients comprising of such families is a lengthy and expensive process - family history needs to be determined as accurately as possible and blood samples need to be taken to analyse each persons' DNA. Processing and analysing DNA is also incredibly expensive, where most whole genome sequencing is not done within the research department that will analyse the resulting genome. Whole genome sequencing typically gets outsourced to dedicated laboratories at a cost per genome. Next Generation Sequencing (NGS) has revolutionized the process of sequencing a persons whole genome and is now the leading method that supports whole genome/exome based research.

\subsubsection{Epidemiology of epilepsy}

Epilepsy prevalence has been measured in various studies [20]-[21] where it ranges between $0.3-0.8 \%$ in developed countries and $0.43-1.4 \%$ in developing countries. In chapter 3 of this thesis a study of epilepsy prevalence in Wales is presented that estimated the prevalence of epilepsy to be $0.77 \%$ of the population, where higher prevalence is found in more deprived areas $(1.13 \%)$ than less deprived areas $(0.49 \%)$, a trend which is also seen in the incidence of new cases of epilepsy.

Incidence of epilepsy is typically highest in children and the elderly, with lower incidence between the age of 18-65, and incidence is double in men over 65 than women over 65 [22]. The majority of incident epilepsy in children are due to genetic factors, where the incidence of epilepsy later in life is due to symptomatic factors as the incidence of neurodegenerative diseases, brain neoplasms and stroke also increase with age. Figure 1.1 presents the "U-shape" curve that describes epilepsy incidence across all ages: 


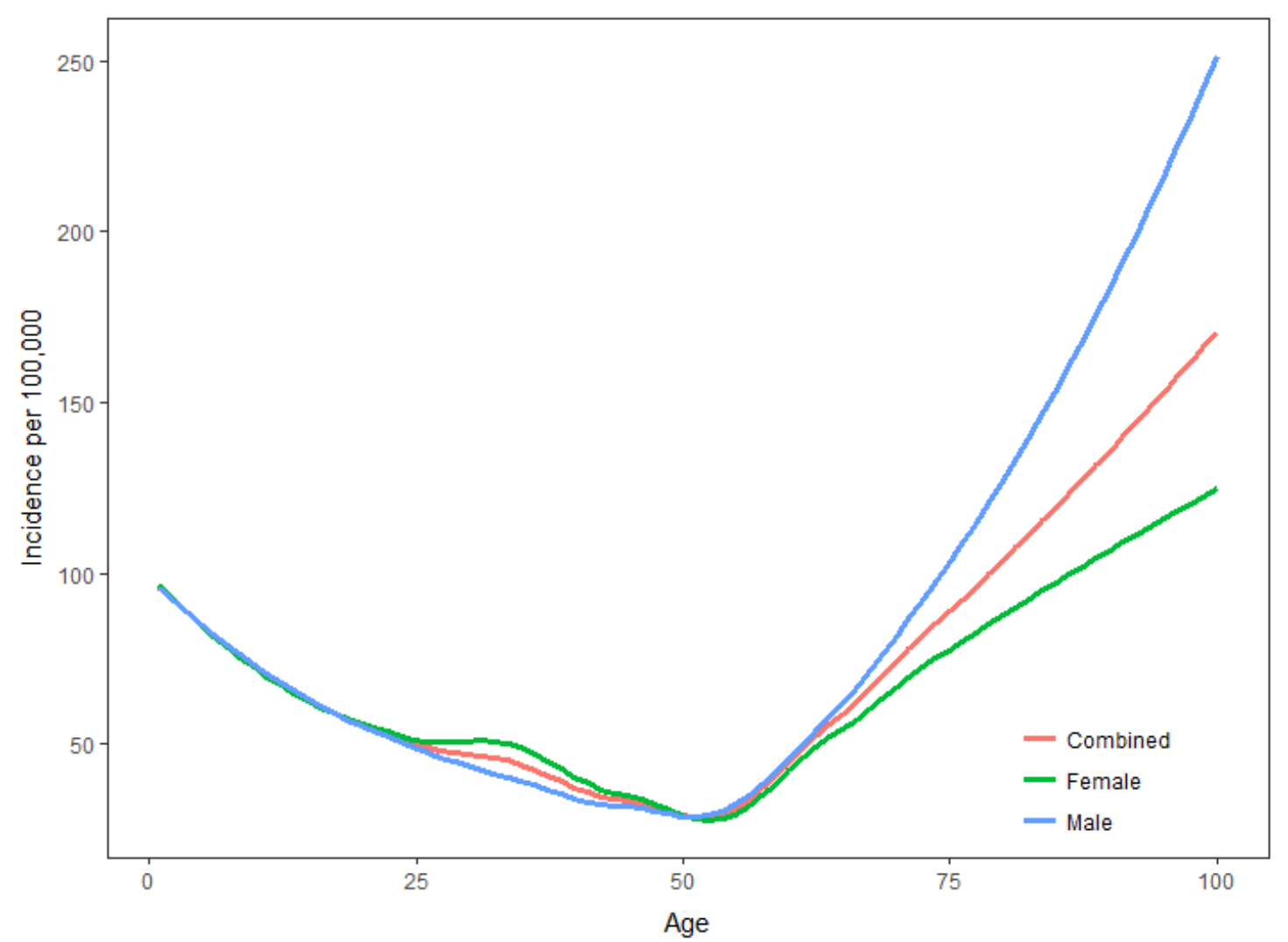

Figure 1.1: Incidence of epilepsy from a study in Iceland showing incidence per 100,000 stratified by age and sex.

\subsubsection{Anti-epileptic drugs}

Anti-epileptic drugs (AEDs) are a group of drugs that aim to suppress unprovoked seizures by suppressing the rapid firing of neurons in the brain during a seizure by binding to specific receptors in the brain and inhibiting voltage dependant sodium currents [23]. AEDs also aim to prevent the spread of seizures in it's early phase to other parts of the brain [24]. Around $50 \%$ of patients treated with AEDs have a 25-50\% reduction in seizures, with other patients with more modest reduction [25]. While AEDs can be effective, around half of epilepsy patients experience adverse effects from a first line AED [26] [26]. Prescribing trends in anti-epileptic drugs have changed in recent years due to evidence of some such side effects of some AEDs.

A study using Welsh GP data held accessed via the SAIL Databank showed that newer AEDs such as Lamotrigine have been prescribed as a first line AED with increasing frequency over a ten year a period between 2000 and 2010, and older AEDs such as Sodium Valproate have seen a reduction in prescribing in women of child bearing age, probably due to evidence suggesting valproate can produce cognitive dysfunction if exposed to children inutero [1] [27]. Figure 1.2 shows prescribing trends of first line AEDs prescriptions in Wales. 


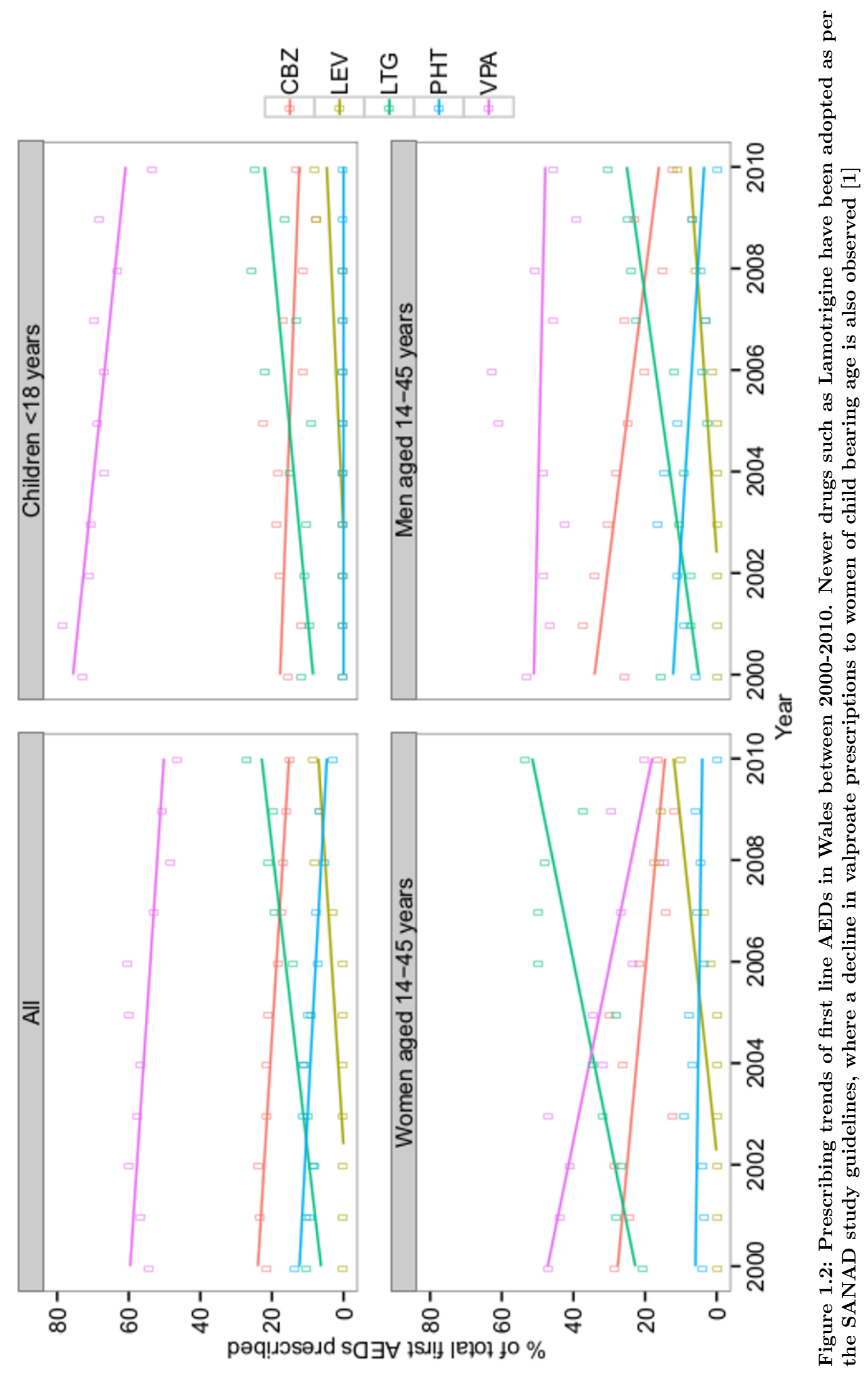


Choice of AEDs to treat seizures doesn't just include seizure control of the individual. Well documented side effects of AEDs include behavioural issues, decline in cognitive function, migraines and psychiatric disorders [28]-[29]. Various AEDs have been studied in relation to weight gain and loss, in which some drugs such as Levetiracetam and Sodium Valproate, while some drugs have been shown to cause weight loss, complicating the issue of AED prescriptions in patients with conditions such as diabetes mellitus [30]- [31].

AED choice is also important when prescribing in pregnant women. Various studies have associated inutero exposure to sodium valproate with a variety of effects on offspring that include reduced IQ, decline in motor and language skills as well as general decline in cognitive abilities [27]-[32]. Recently a Danish study found that children exposed to valproate intuero perform worse then their peers in national tests [33]. Chapter 4 in this thesis presents a study using Welsh Key Stage 1 education tests and AED prescribing data in pregnant women that found inutero exposure to sodium valproate and AEDs in combination are associated with decreased educational attainment in children aged 7 [3].

\subsubsection{Burden and impact of epilepsy}

The Global Disease Burden Study has estimated that epilepsy contributes to $1 \%$ of all days lost due to ill health and that on average epilepsy forms $0.5 \%$ of total disease burden as measured by the Disability Adjusted Life-Year (DALY) score. [34]. Epilepsy places a huge burden on those who suffer unprovoked seizures on a daily basis, a burden which is also shared by the relatives and peers of someone with epilepsy [35].

Epilepsy is a condition associated with a range of co-morbid conditions. Around $40 \%$ of adolescents with epilepsy also have an additional neurological condition and 1 in 4 persons with epilepsy of any age has a learning disability [36]. Behavioural issues are prevalent in children with epilepsy exhibited both in school and at home [37] [38]. Children with epilepsy are a stigmatized group and are twice as likely to be bullied at school than their peers [39], and a qualitative study of children with refractory epilepsy viewed seizures as a barrier to a normal life [40].

Seizures disrupt short term information storage, especially nocturnal seizures when most memory consolidation takes place. The physical impact of seizures on the brain is associated with memory loss in people with epilepsy ranging from concentration issues to chronic forgetfulness [41]. Auras occurring before and during a seizure and other lapses of concentration contribute to poor recall of which 1 in 4 people cannot recall experiencing auras or lapses in concentration [42] and people with epilepsy 
fail to document around $50 \%$ of recent seizures. Poor memory loss therefore also contributes to difficulties in learning along with increased risk of being born with learning difficulties.

Around $80 \%$ of epilepsy is prevalent in developing countries in which there exists not only a barrier to effective care and seizure treatment, but there is an undeniable trend in epilepsy, social deprivation and social stigma [43] [44]. People with epilepsy are likely to experience prejudice and discrimination in all walks of life as well as being at high risk of abuse and violence [45] [46]. Young people with epilepsy are often discouraged from pursuing their chosen career path [35] and face discrimination in life ranging from diminished access to various insurance schemes and employment opportunities [47]. One survey of employers found that $16 \%$ felt that they didn't have jobs for someone with epilepsy and $21 \%$ considered employing someone with epilepsy as "a major issue" [48].

Complimentary to the fact that epilepsy is more apparent in developing nations, lower socio-economic status is a risk factor for epilepsy in adults [49] and the various social struggles people with epilepsy face is a strong argument for epilepsy causing social drift. Multiple studies have associated social deprivation with epilepsy in both new cases of and existing epilepsies [50] [51]. There are two main hypotheses for social deprivation in epilepsy; social causation and social drift. Social causation in epilepsy could be explained by factors associated with both deprivation and causes of epilepsy, namely such as perinatal hypoxic injury, head trauma, and cerebrovascular disease [21] [52] [53]. Social drift is hypothesised to explain some of the high deprivation seen in people with epilepsy for various reasons related to social stigma and discrimination in employment. Chapter 4 presents a study of social deprivation in Welsh patients with epilepsy and in both newly diagnosed patients and patients with existing epilepsy.

\subsection{Big data and patient records as a resource for research}

In this section, patient records and large linked datasets are presented as a method for researching the epidemiology and burden of epilepsy for large cohorts. The SAIL Databank is presented as such a resource that is utilized in this thesis to conduct population level epilepsy research in Wales. 


\subsubsection{Electronic Healthcare Records}

Up to 1990 in the UK, patient records were largely paper based. Large scale Electronic Healthcare Record (EHR) linkage as a research method has grown as a direct result of embedding computer systems in primary care to make the transition from paper based records to electronic records. In 1987, two GPs from Egton, Yorkshire Dr Peter Sowerby and Dr David Stables formed the Egton Medical Information Systems Group (EMIS) to develop software that could capture paper based records into electronic format. The result was a commercial rollout of the EMIS software in 1990, and EMIS, as well as many other clinical audit systems that followed, enabled GPs to capture routinely collected information at point of care and have instant access to those records at a later time. As thousands of GP practices started to employ such systems, the resulting records were quickly used to monitor trends and performance of GP practices [54] and measure outcomes in patient care. After years of patient interactions with GPs being recorded electronically, large scale retrospective epidemiological were made possible because access to patient records were much faster compared to sourcing paper based records.

Using the first EHRs as a form of research was a success mainly because the data collected by GPs to inform patient care was mutually beneficial to inform public health. GPs not only recorded patient details important to building a picture of patient care such as diagnoses and medication, they recorded patient details using clinical coding systems to summarise their interactions. In 1990 the READ clinical coding system had matured for 8 years and was considered for use in computerised coding systems [55]. Developed from the early 1980's onwards and still being developed today, Dr James Read built a clinical coding system consisting of 250,000 codes that could be used to describe the details of a patient's medical notes. While clinical coding systems such as ICD have existed for over a hundred years [56], the READ code system was the first in the UK that could classify disease, symptoms, prescriptions and referrals in one heirachy. The real advantage of using computerised records and embedding coding systems such as READ used in GP practices, and ICD-10 used in secondary care is how EHRs can be queried using computer languages such as SQL. Patient information can be rapidly accessed and partitioned by these coding structures to create cohorts of certain disease or medication retrospectively. This is by far more efficient and reliable than having to process free text such as medical notes manually, a problem still being solved today through Natural Language Processing - discussed later on in this chapter. The electronic patient record, when combined with clinical coding systems are a potential data source for fast, large scale research.

However, a fundamental understanding of the purpose and context of why and 
how EHRs are recorded must be taken into account if intended for use in research. Patient records are designed to be collected at point of contact which usually means a consultant or GP. The records are therefore a reflection of how a patient should be cared given their presentation at point of contact, and are certainly not designed for research purposes. For example, a record of a prescription does not necessarily mean the patient has adhered to a treatment plan, or a diagnosis code used by a GP could indicate a diagnosis subject to a specialist referral rather than a definite diagnosis. Even when administrative staff take more of a role in entering details of patient records, the ability to generate factually correct patient records relies on communication with consultants or specialized training to translate consultants finding into clinical codes. While technology moved forward the ability to create patient records more efficiently, the sources of error remain the same as when paper records were used. For research purposes this broadly means that any conclusions are limited by the quality of data entered into patient records, or to put more crudely garbage in, garbage out.

Aside from data entry being influenced by how patient records will be used in-house by medical professionals, incorrect data is ingrained in patient records. Human error is a factor in any data entry tasks, but data entry in a live healthcare setting is arguably more difficult than most data entry tasks. Clinical coding is a fast growing profession within secondary care that requires a strict set of exams to qualify as a clinical coder. Their job is to sift through consultants, surgeons, junior doctors and pharmacists notes to build a patient profile and turn them into discrete episodes of care described by ICD-10 and OPCS-4 codes. This process is detective-like by nature, often having to piece together conflicting medical opinions, sifting through short-hand patient notes and assigning a subset of the 16,000 codes ICD-10 codes to describe disease and morbidity, and OPCS-4 codes to describe operations [57]. The potential for error is large without the high standard of training and continuous communication with the various healthcare professionals that are responsible for treating the patients. In contrast to the use of clinical coders, GPs are expected to enter data into patient records at point of care. While clinical audit systems such as EMIS aim to help GPs accomplish this task, it is incredibly difficult for GPs to have a working knowledge of the 250,000 code list in the READ code system while entering data and caring for patients in an average consultation time of just 11 minutes. Under these pressures it is easy to imagine why GPs may be forced to cut corners or omit certain aspects of coding. This can lead to systematic error in coding such as using codes that do not accurately describe the patient or limiting their use of READ codes to a very small subset regardless of what the patient presents with.

The electronic patient record has however become the cornerstone informing medical 
practice through either research or immediate feedback of data at point of care. The explosion of research based on information in EHRs has no doubt furthered the case for more emphasis to be placed on accuracy and maintenance of EHRs and making EHRs as robust as possible. There have been incentives such as the Quality of Outcomes Frameworks (QOF) that pay GPs to use a wide variety of READ codes in clinical practice in which the effectiveness of well coded EHRs post-QOF showed reduction in mortality, hospital admissions and the improvements in the management of chronic conditions such as diabetes [58] [59].

Consequently, the demand for a patient record for both healthcare and research has evolved beyond what is recorded in primary and secondary care. Various national health registers and audits ranging from the Office of National Statistics Deaths and Births register, Congenital Anomaly Register and Information Service (CARIS) [60], Welsh Cancer and Intelligence and Surveillance Unit (WCISU) and various biobank datasets from clinical trials all feed into the patient record. Social care datasets and tertiary health programs provide useful measures of patient care outside of first and second line services, as well as administrative and demographic data such as the Welsh Demographic Service to explore geographical and social deprivation effects on health. Perhaps the most exciting addition to the patient record is genetic data due to the potential for deeper understanding inherited disease and the opportunity to develop personalised medicines. The patient record is beginning to include information that is not even found in database format or collected via traditional auditing methods - namely free texts such discharge letters and consultant reports that contain far richer data than any of the datasets previously mentioned, if it can be processed. Any dataset that can feed into the patient record is beneficial, especially for research, a prospect which becomes eve more powerful when such datasets are successfully integrated together.

\subsubsection{The SAIL Databank}

The linkage of big data is a corner stone of public health research. The potential to mine patient profiles from national datasets produces novel research that directly impacts policy. While randomized control trials are the benchmark for studying health interventions and drug use, retrospective, longitudinal studies produced from linked data is much cheaper, faster and statistically more powerful. There are many established data centers that take advantage of linking routinely collected data, but the largest government funded initiative for using data linkage in health research in the UK is the Farr Institute of Health Informatics Research. The Farr Institute was a collaboration of 21 academic and health institutions in the UK, where the four

main data centers are co-ordinated through The Health e-Research Center at the 
University of Manchester, University College London, University of Dundee and the SAIL Databank at Swansea University. Since then the Health Data Research (HDR UK) initiative was set up to continue innovation in big data linkage for research, of which new centers such as the Sanger Institute are now included as a means to bring genetic data to the patient record.

The SAIL Databank is a repository for national health datasets in Wales, hosted at the HDR UK cite at Swansea University, that contains linked anonymised health records at a patient level [61] [62]. Developed in 2006, the SAIL databank aimed to take advantage of emerging technologies to capture patient level data and provide a platform to link datasets on a large scale. National datasets such as primary and secondary care, mortality and birth records, geographic and socio-economic status all have routinely collected data that date back 25 years and when linked together produce research potential greater than the sum of it's parts. Figure 1.3 shows the different types of data held in the SAIL databank:

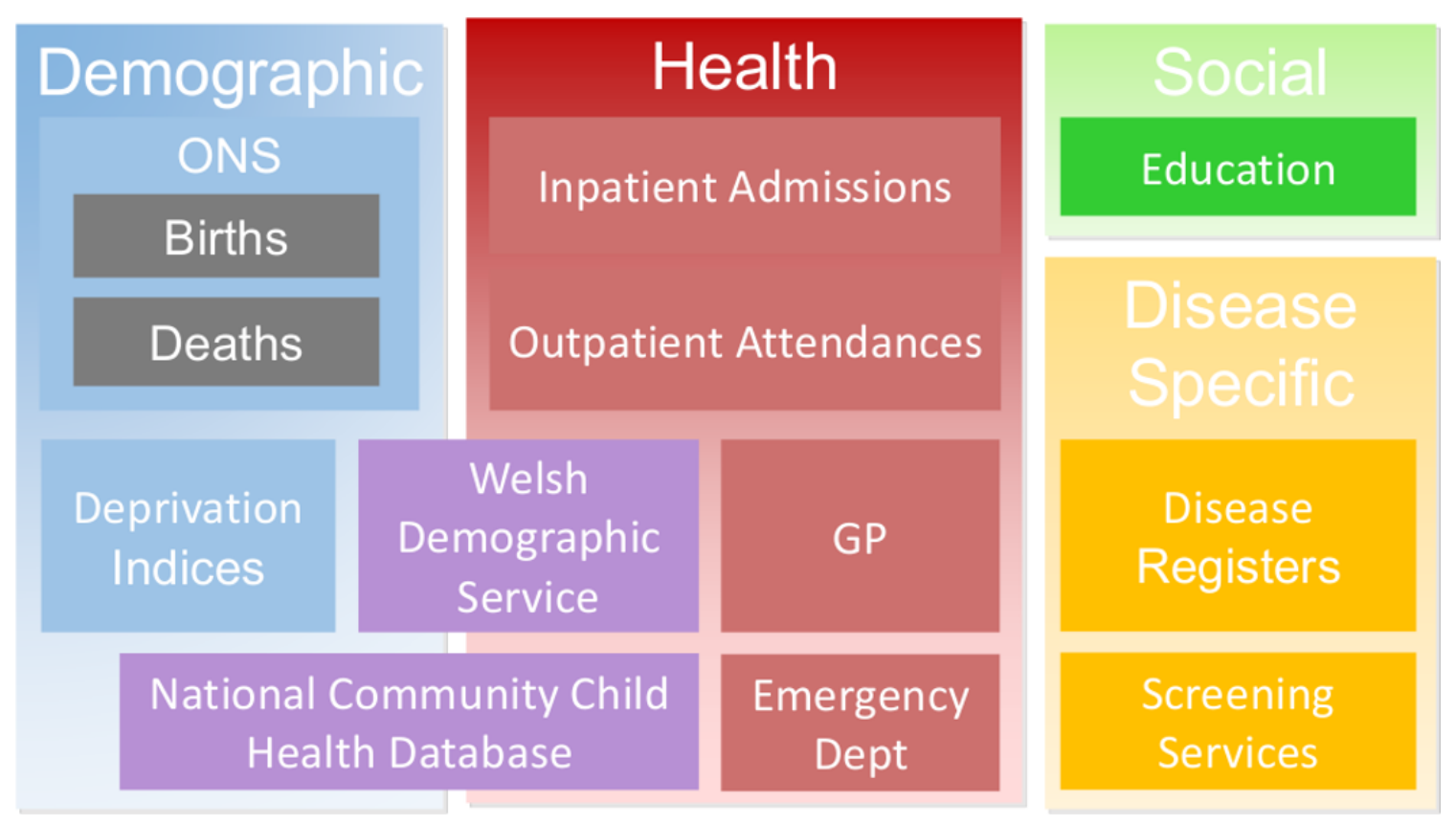

Figure 1.3: The core SAIL datasets. Each dataset can be linked anonymously via an encrypted NHS number

\subsubsection{Anonymous patient records}

Datasets held in SAIL are anonymised using a split file procedure. Each data provider splits their dataset into 2 parts - one containing all demographic data which is sent to a trusted third party (TTP), and the other containing only clinical data that is sent directly to SAIL. An internal system ID that bears no relation to the patient ID is the only field shared between the split files. The TTP, in this case the National Welsh 
Information Service and SAIL each encrypt the split datasets, and are then combined using a shared encryption key. The result is a completely anonymised dataset that can be linked to all other datasets within SAIL.

Users can assess the repository through the SAIL gateway - a remote server with a Graphical User Interface (GUI) front-end. The SAIL databank is powered by an IBM Blue-C supercomputer which provides extremely fast database queries, capable of completing queries on databases of millions of records within seconds. The data is warehoused and made available as a repository of relational databases, in which the data can be queried using IBM DB2 Structured Query Language (DB2 $\mathrm{SQL})$. As data is anonymised there is no ethical approval needed to query data. However an independent Information Governance Research Panel (IGRP) consisting of multi-disciplinary professionals in the field of health care and health care research exists, to which project studies plans are scrutinized to ensure the research question is valid and answerable using SAIL data, as well as ensuring that no individuals can be identified. Once a project is approved, data can be requested out of the SAIL gateway. An internal team of researchers view all outputs to ensure no sensitive data leaves the SAIL gateway.

\subsubsection{SAIL studies}

The SAIL databank has been used in a diverse range of healthcare studies. The main type of studies conducted are retrospective longitudinal studies that take advantage of millions of person-years of data across multiple health datasets, although follow up studies from patient recruitment have also been carried out.

\section{Child Health and Births}

The Wales Electronic Cohort for Children (WECC) is an e-cohort of children in Wales set up to study a range of social and environmental determinants and outcomes of child health. The WECC cohort is the largest e-cohort for children in health $(804,290$ children, 375,025 mothers between 1998-2008) and was built from routinely collected data in the SAIL Databank [63]. Several studies have used the WECC cohort to research health and social outcomes in children. One study found a 4 fold increase in hospital admissions for children born at a gestational age of 33 weeks (41.5 per 100 child years) compared to gestational age of 40 weeks (9.8 per 100 child years) [64]. The impact of skull fractures and inter-cranial injury was associated with poorer academic performance in Key Stage 1 assessments compared to a control group, and a higher risk of hospital admission was observed in children with a mental health

disorder or living with parents that had an alcohol misuse problem recorded in GP 
records [65] [66]

The Congenital Anomaly Register and Information Service for Wales (CARIS) dataset was linked to ONS birth records in the SAIL Databank to estimate the prevalence of Turner Syndrome to 1 in 4901 female births [67]. An example of bespoke datasets being linked to the SAIL Databank is a study using data from the Singleton Hospital Maternity Ward at Swansea University that were linked to primary and secondary care datasets to study the relationship of BMI during pregnancy and health utilization. Based on data of 484 pregnancies it was found that healthcare costs during pregnancy was $37 \%$ higher in obese women compared to those with normal weight. Demographic data was linked to Key Stage 1 education data showing that a clear trend in reduction of educational attainment was seen with increasing numbers of house moves, even in children that moved prior to the Key Stage 1 assessment period ( $<5$ years of age) [68].

\section{Mental Health}

A cohort ascertainment study using GP records in the SAIL Databank specified sets of READ codes to define anxiety and depression. Using results of the Caerphilly Health and Social Needs Survey (CHSNS), combinations of depression and anxiety diagnoses, medication and symptoms showed that high positive predictive value could be achieved, but it is likely that depression and anxiety are under reported in GP records[69]. A further analysis of GP recording of depression showed that diagnoses recorded in GP settings have declined while antidepressant prescribing has increased in adolescents, indicating GP coding habits change over time and highlights the importance of understanding reference data for epidemiology studies from routinely collected healthcare data [70].

The Suicide Information Database Cymru (SID-Cymru) was set up using mortality and secondary care data in the SAIL databank to identify 2664 cases of suicide in Wales between 2003-2011 [71]. The SID-Cymru dataset was used to obtain suicides following alcohol related emergency admissions to hospital which showed that women were at double the risk of suicide than men and that $10 \%$ of suicides took place within 4 weeks of admission [72].

\section{Multiple Sclerosis}

The UK MS Register was set up to obtain rich patient and clinically reported information on patients with MS. Patients can upload their medical data via web forms and social media, to which they have consented for this information to be used in healthcare research. All data is hosted by the SAIL Databank and is available 
to be linked to existing datasets in SAIL [73]. Responses from the web-portal were used to assess how MS patients fair on the Hospital Anxiety and Depression Scale (HADS), where HADS measures anxiety and depression using a scoring system (0-7 neither reported, 8-10 mild, 11-14 moderate and 15-21 severe). The results of 4178 respondents showed that the median HADS score was 15.7, with over half of respondents score $\geq 8$ for depression and just under half scoring $\geq$ for anxiety [74]. A follow study correlated increasing prevalence of anxiety and depression with increasing physical disability where anxiety or depression was reported in $38 \%$ of people with mild physical disability, $66.7 \%$ with moderate physical disability and $71 \%$ with high disability [75]. Patients in the MS Register also answered a survey to determine their generic health status from an EQ-5D in which people with MS scored $22 \%$ less than the UK mean of $82 \%[76]$

\section{Diabetes}

A study of ONS birth records in the SAIL Databank identified 1250 pregnancies where the mother had existing diabetes and 1358 gestational diabetes in which contrary to hypotheses of "obesity programming" in children born to mothers with diabetes, little evidence of this effect was found unless the mother was also found to be in the highest weight tertile during pregnancy [77]. 1577 children between the ages 0-15 with type-1 diabetes from the Brecon Group Register were linked to hospital admissions in SAIL. The study found a $480 \%$ incidence of hospital admissions in which the incidence rate decreases $15 \%$ with each increasing 5 year age band [78].

HbA1c measurements recorded in GP records were compared before and after an incident stroke in patients with existing type-2 diabetes. 1741 diabetes patients were identified having HbA1c measurements before or after an incident stroke and were age and sex matched 1:4 to a control group of patients with diabetes that had not had a stroke. On average there was a $7.5 \%$ decrease in HbA1c measurement after the incident stroke, indicating increased monitoring post-stroke in patients with diabetes may result in better glycemic control [79].

\section{Cardiovascular Disease}

A study linking hospital records for patients admitted for acute myocardial infarction (AMI) $(\mathrm{n}=30,633)$, stroke $(37,888)$ and sub arachnoid haemorrhage (SAH) (1753) to ONS Death certificates in the SAIL Databank explored the effects of social deprivation on 30 day mortality following admission. Baseline 30 day mortality rates for AMI, stroke and SAH were $14.3 \%, 21,4 \%$ and $35.6 \%$ respectively, however a $24 \%, 24 \%$ and $32 \%$ increase in mortality was observed when comparing the lowest deprived quintile to the highest [80]. Statin use in patients presenting with incident acute coronary 
syndrome (ACS) to the Cathlab Unit in Morriston hospital was studied to investigate if guidelines were being taken up to prescribe statins post ACS. $80 \%$ of patients were prescribed statins with simvastatin most common, however only $38 \%$ were prescribed a high dose, leading to the conclusion that statin use post-ACS is under utilized in Wales [81].

Cathlab data for patients with aortic stenosis was used to show that Transcatheter Aortic Valve Implantation (TAVI) was more effective than being medically managed in terms of prospective health utilisation and mortality over a 2 year period. Mortality rates were half that in the TAVI group (19.2\% vs 41.7\%) and experienced less hospital stay length (0.86 vs $1.84 \%$ person days per year) and costs within primary and secondary care was half that than the medically managed group (£6059 vs £11001) [82]. In contrast to many studies indicating worse health and social outcomes in people from deprived areas, including many SAIL studies, a study of patients with coronary artery disease (CAD) found no health utilization or treatment inequality across Welsh Index of Multiple Deprivation deciles [83].

\section{Epilepsy}

An algorithm using GP records was developed to identify cases with epilepsy using a combination of AEDs and epilepsy diagnosis codes. This algorithm was used to study prescribing trends of first line AEDs in people with epilepsy between 2000-2010. The study showed a sharp decrease in Sodium Valproate prescribing to women of child bearing age, and that recent guidelines from the MHRA to prescribe lamotrigine as a first line AED prescription had been taken up in Wales. [1]. The effects of sodium valproate, lamotrigine, levetiracetam, topiramate and carbamazepine on weight change were explored in a cohort 1423 epilepsy patients. Significant weight gain $(+1 \%$ body weight) in levetiracetam and significant weight loss $(-2.62 \%$ body weight) in topiramate was found, where the other 3 AEDs showed no significant change in weight [84].

A collaborative study between Manchester University and Swansea University found that people with epilepsy are twice as likely to die from suicide than people without epilepsy, are 3 times more likely to die accidentally, are 5 times more likely to die of accidental medication poisoning and are 3.5 times more likely to die of intentional medication poisoning [85]. Emergence admissions of patients with epilepsy who had attended ED for reasons specific to epilepsy were studied in which social deprivation and living alone were identified as risk factors for ED attendance and of these patients, and psychiatric co-morbidities and learning disabilities than epilepsy patients who had not attended ED for epilepsy specific reasons [73]. 


\subsection{Natural Language Processing: Using clinic letters as a data source for research}

Natural Language Processing (NLP) is a multi disciplinary field of linguistics and computer science that aims to construct computer algorithms that can automatically parse unstructured text into more manageable forms. Typically these algorithms are aimed at unstructured texts reflective of human language such as clinic letters, and a suitable task for NLP might be to automatically extract letters where a diagnosis of a certain disease is written in the text. At present, only patient records stored in structured databases are immediately accessible for healthcare research and epidemiology. For decades researchers have been able to take advantage of the codified format of these datasets to make quick gains in epidemiological research. ICD-10, READ and SNOMED-CT codes can quickly be used to manipulate cohorts of patients with structured query languages, but other forms of medical information are slowly starting be adopted into a big data patient record. While huge efforts go into producing an organised patient record at point of care, a large amount of patient information is still recorded only by free text. These include consultant notes, discharge letters, GP correspondence, radiology reports and even structured databases may contain so fields that store free text. Free text in is seen as a rich source of patient information not found in the structured patient records. but remains a challenge to bundle the information into a database format that lends itself to data manipulation. While there is no agreed way to process free text correctly, a number of scientific disciplines have come together to address this problem.

Natural Language Processing (NLP) is a discipline that aims to process free text into easily accessible information, such as a summary or database. NLP draws upon advances in statistical theory, machine learning, artificial intelligence and computer science to create programs or models that understand the nuances of human (natural) language.

Early NLP techniques developed in the 1960's and 1970's used rule sets and pattern matching techniques to infer meaning from text [86],[87],[88]. These early works focussed on creating extensive hand crafted rules that used the relationships between text units such as nouns, verbs and adjectives to extract structured items of information. Using complicated human rule sets to parse language relies solely

on human knowledge of text to predict patterns in advance that would capture items of interest and the context they are found in. The scale of this problem has required more sophisticated approaches to be developed. The advent of machine learning in the 1980's provided ways to parse text not through fixed sentences, but through teaching 
a computer to learn the role of each word in a sentence [89]. Part Of Speech (POS) tagging abandons highly conceptual human rule sets declared to a computer algorithm prior to analysis, and analyses the relationships of verbs, adjectives and nouns in relation to a dictionary of words of interest i.e. medical terminology. Machine learning allows a ground truth such as phrases known to confirm disease or symptom, be used to train an algorithm to recognize the patterns between each word in a sentence and words of interest. This machine learning approach does not rely on complex rules sets, but rather learns the language used to describe cohort characteristics. Structured concepts such SNOMED terminology can be "mined" out of free text, including information that would go unseen or undefined by prior rule sets. In the methods chapter various NLP techniques are described and tested to define characteristics about patients with epilepsy from clinical notes.

\subsubsection{Part of Speech Tagging}

Part of Speech (POS) Tagging involves assigning word classes such as verbs, nouns, adjectives as well as more complex classes such as qualifiers, prepositions and adverbs to tokens in text. This cannot be achieved by a simple lookup because words can be assigned as different word classes based on context. For example the word haemorrhage in the phrase "there is a chance she will haemorrhage" is classed as a verb, but used in the phrase "she has had a haemorrhage" it is classed as a noun. Assigning the correct word class for each token is crucial to NLP tasks such as information extraction where word classes can be built into rules or machine learning processes as basic building blocks that help identify concepts within text.

The development of POS tagging has relied on analysing large corpora of many of documents such as the Brown Corpus [90] and the Cobuild project [91] so that common data sources can be used to both develop and benchmark POS tagging algorithms. The Brown Corpus consisted of 1 million words of English prose from randomly selected scientific publications and was used to develop custom tagsets to encapsulate detailed tags that extend beyond basic word classes. The Brown Corpus was manually annotated over many years and served as a target tagset for computerized algorithms. The first attempt to develop a computerised algorithm from the Brown Corpus used human logic such as an article followed by a noun can occur i.e. "Dr House", but in general an article followed by a verb does not occur. This approach yielded an initial accuracy of $70 \%$ [92].

Both larger corpora and machine learning approaches were adopted to increase POS Tagging accuracy. Hidden Markov Models - a probability state classifier was tested on the Lancaster-Oslo-Bergen Corpus [93] of British English. Hidden Markov 
Models were able to take bi-grams, tri-grams and n-grams as input from the manually annotated POS tags to calculate the probability of a TAG for each element of an n-gram. This was a popular method of POS-tagging which used the scalability of dynamic programming to produce fast and accurate taggers [94], [95], [96]. Some of the most widely used POS taggers are rule-based. The Brill tagger [97] uses a set of rules that recursively updates tags during repeated phases. An initial phase is run generating most likely tags, in which set of conditions are imposed to correct each tag. This process is repeated until a threshold is met in terms of the proportion of tags corrected. The Penn Treebank POS tagset project annotated POS tags over a corpus consisting of 4.5 million American-English words from the Brown Corpus and the Wall Street Journal using a combination of Church's PARTs method [94] and manually correcting any errors, in which this method was measured to be twice as fast as a fully manual annotation method [98]. While most POS tag studies up to this point published an automated algorithm for POS tagging, the original Penn Treebank paper does not describe such a method but provides a widely used benchmark corpus for training POS taggers (machine learning, rule based or hybrid) for unseen samples of text. Table 1.4 shows the PENN treebank tags that are commonly used to tag texts:

Table 1.4: A table of PENN treebank POS tags. https://www.ling.upenn.edu/courses /Fall_2003/ling001/penn_treebank_pos.html

\begin{tabular}{llll}
\hline Tag & Description & Tag & Description \\
\hline CC & Coordinating conjunction & PRP\$ & Possessive pronoun \\
CD & Cardinal number & RB & Adverb \\
DT & Determiner & RBR & Adverb, comparative \\
EX & Existential there & RBS & Adverb, superlative \\
FW & Foreign word & RP & Particle \\
IN & Preposition or subordinating conjunction & SYM & Symbol \\
JJ & Adjective & TO & to \\
JJR & Adjective, comparative & UH & Interjection \\
JJS & Adjective, superlative & VB & Verb, base form \\
LS & List item marker & VBD & Verb, past tense \\
MD & Modal & VBG & Verb, gerund or present participle \\
NN & Noun, singular or mass & VBN & Verb, past participle \\
NNS & Noun, plural & VBP & Verb, non-3rd person singular present \\
NNP & Proper noun, singular & VBZ & Verb, 3rd person singular present \\
NNPS & Proper noun, plural & WDT & Wh-determiner \\
PDT & Predeterminer & WP & Wh-pronoun \\
POS & Possessive ending & WP\$ & Possessive wh-pronoun \\
PRP & Personal pronoun & WRB & Wh-adverb \\
\hline
\end{tabular}




\subsubsection{Shallow parsing}

Shallow parsing or "text chunking" is the process of grouping tokens into n-grams such as sentences, phrases as well as further sub-units commonly known as chunks. The sentence "The radiologist was able to observe the tumours visible against the background" can be split up into five chunks "The radiologist" "was able to observe" "the tumours" "visible" "against the background", in which one chunk usually provides context to other chunks in close proximity. Chunks are defined as sub-units of text that contain a "potential governor" - a handle placed at the rightmost part of a chunk [99]. The potential governors in the above phrase would be "radiologist", "observe", "tumours", "visible", "background".

The Brill POS tagger algorithm and PENN treebank tagsets were used as input to develop a rules based noun phrase chunker that defined noun phrase chunks such as "she has epilepsy" and "her seizures are frequent" [100]. Further algorithms were introduced to classify verb phrases (VP), prepositional phrase (PP), adjective phrases (ADJP) and adverb phrases (ADVP) [101]. The Conll-2000 shared task [2] defined 13 different chunk types as targets for classifiers to learn by providing a fully annotated version of the Penn Treebank Corpus. These chunk types are shown in table 1.5. 
Table 1.5: The chunk types defined as part of the CONLL-2000 shared task [2]

\begin{tabular}{|c|c|c|}
\hline Chunk Type & Definition & Examples \\
\hline NP & $\begin{array}{l}\text { Noun phrase - phrases beginning with a } \\
\text { noun }\end{array}$ & $\begin{array}{l}\text { "Mr Jones", "He was", } \\
\text { "a year" }\end{array}$ \\
\hline VP & $\begin{array}{l}\text { Verb phrase - phrases beginning with a } \\
\text { verb }\end{array}$ & $\begin{array}{l}\text { "may want to increase", } \\
\text { "could be a", "broke the" }\end{array}$ \\
\hline $\mathrm{PP}$ & $\begin{array}{l}\text { Prepositional phrase - a phrase to place } \\
\text { context to nouns }\end{array}$ & $\begin{array}{l}\text { "at night", "because of } \\
\text { ", "due to" }\end{array}$ \\
\hline ADVP & $\begin{array}{l}\text { Adverb phrase - pre or post modifier to a } \\
\text { verb or verb phrase }\end{array}$ & $\begin{array}{l}\text { "very well", "overdosed } \\
\text { earlier", "quickly" }\end{array}$ \\
\hline SBAR & $\begin{array}{l}\text { Subordinated clause - conjunction between } \\
\text { other phrase types }\end{array}$ & $\begin{array}{l}\text { "so that", "even if", } \\
\text { "until" }\end{array}$ \\
\hline ADJP & $\begin{array}{l}\text { Adjective phrase - phrases beginning with } \\
\text { an adjective }\end{array}$ & $\begin{array}{l}\text { "upset with her seziures", } \\
\text { "prolapsed disk" }\end{array}$ \\
\hline PRT & $\begin{array}{l}\text { Particles - verb/adverb attached to non } \\
\text { inflected words }\end{array}$ & $\begin{array}{l}\text { "look up", "on and } \\
\text { off", "in and out", "get out" }\end{array}$ \\
\hline CONJP & $\begin{array}{l}\text { Conjunction phrase - multiword } \\
\text { conjuntions to list additional phrases }\end{array}$ & $\begin{array}{l}\text { "as well as", "not } \\
\text { only", "but also" }\end{array}$ \\
\hline INTJ & $\begin{array}{l}\text { Interjection - phrase containing an } \\
\text { ubrupt remark }\end{array}$ & $\begin{array}{l}\text { "oh", "alas!", } \\
\text { "good grief!" }\end{array}$ \\
\hline LST & List marker - denotes a list & $\begin{array}{l}\text { "firstly...", "1.", } \\
\text { "lastly", "a,b,c" }\end{array}$ \\
\hline
\end{tabular}

Eleven algorithms were submitted to the Conll-2000 shared tasks and the most accurate algorithm achieved an F-score of 93.4\% [102]. Shallow parsing algorithms have usually focussed on employing machine learning and statistical learners such as Hidden Markov Models, Support Vector Machines, Naive Bayes and Conditional Random Fields [103], [104], [96], [105].

\subsubsection{Named Entity Recognition}

Named Entity Recognition (NER) is the process of tagging specific words or phrases and labelling them into entities and such as persons, addresses, identification numbers, diseases, symptoms or medication. NER tasks usually involve mapping entities to a user specified dictionary. NLP tasks focussing on healthcare typically map entities to medical ontologies such as as MetaMap, The Unified Medical Language System, SNOMED-CT and ICD-10 [106],[107]. NER has advanced significantly since scientific events and competitions were set up in the 90's, with the Messaging Understanding Conference (MUC-6) [108] set up specifically to bring together groups researching 
NER. Many other annual conferences such as HUB-4 [109], the Information Retrieval and Extraction Exercise (IREX) conference for Japanese NER [110] and the Automatic Content Extraction (ACE) [111] conference have since been set up to maintain a focus on NER and are still running today.

The main challenges for NER tasks are:

- Word ordering - the order of words within a phrase can change the meaning of entities to be labelled. For example the phrase "she had a blood pressure check" identifies that this person has had a blood pressure measurement, but "to check her blood pressure" implies this is something that should happen in the future and not be assumed this person has had a blood pressure measurement at the current time.

- Inflexions - suffixes and prefixes can change to indicate a different meaning for a word. For example "big/biggest", "vomit/vomited", "informed/uninformed" etc.

- Homopgraphs - the same words can have different meaning. The word "fine" can mean something is normal or it can describe a procedure or wound. Research in word-sense disambiguation is dedicated to addressing homographs.

- Synonymy - the opposite of homographs in that multiple entities can mean the same concept i.e. "Focal seizures/ partial seizures".

- Negation - certain trigger words such as "not/no/never/unlikely" indicate that an entity has not been found, and therefore should not be attributed to a positive finding in text. It is common to tag entities with negation status.

- Word relationships - and extension of negation. The surrounding words around an entity dictate it's context. For example the phrase "if the results are positive, prescribe Lamotrigine" suggest a prescription of Lamotrigine is only positive given the context of a result prior to the word Lamotrigine.

- Temporal qualifiers - describing a temporal feature attached to an entity involves measuring the proximity and order of trigger words relating to past, present or future tenses. 


\subsubsection{NLP tools and software}

Table 1.6: A list of common NLP software

Toolkit Description

Stanford Natural Language

Processing Toolkit [112]

Open source Stanford CoreNLP toolkit. Contains standard

Processing Toolkit [112]

NLP applications (POS taggers, chunker, NER). Developed

NLTK [113] by Stanford University.

Apache UIMA [114]

Natural Language Toolkit developed in Python

Apache OpenNLP [115

Open source Java based Unstructured Information Management Application developed by the Apache Software Foundation

Open source NLP toolkit adopted by the Apache Software Foundation and developed by the open source community

Open source healthcare information extraction system developed by the Mayo Clinic

The General Architecture for Text Engineering. An open source NLP architecture developed at Sheffield University. Contains a variety of standard NLP applications as well as user contributed plugins. Has a rich GUI and can be run in embedded systems.

IBM WATSON Content A proprietary NLP product produced by IBM. Makes use of Analytics [118]

Spacy [119] the UMIA framework and has a rich GUI.

\begin{tabular}{ll} 
Open NLP (R package) [120] & $\begin{array}{l}\text { An R package that interfaces to the Apache OpenNLP tools } \\
\text { written }\end{array}$ \\
TM (R package) [121] & A text mining framework written in R. Contains many NLP \\
applications & A UIMA framework for executing rule based scripts for NLP \\
Apache UIMA RUTA [122] & applications \\
Gensim [123] & A python library for vector space modelling of large text \\
Word2Vec [124] & An algorithm to produce words embeddings for topic \\
& modelling. Developed at Google trained on a Google News \\
corpus. & An algorithm developed at Stanford University to produce \\
GloVe $[125]$ & word embeddings \\
\hline
\end{tabular} 


\subsubsection{Validating NLP algorithms}

Any algorithm proposed for an NLP task can be measured against a human annotator, or multiple annotators. Annual shared tasks such as CoNLL specify a problem statement as well as providing or using large, annotated corpora to compare against algorithm submissions. Comparison between a human annotator and an NLP algorithm on a binary classification results in true positives (target class labelled as target class by algorithm), false positives (non-target class labelled as target class), true negatives (non-target class not identified as target class) and false negatives (target class not identified as target class). By assigning each item identified by both human annotator and an algorithm as a true positive, true negative, false positive or false negative, the overall accuracy can be measured in various ways. In NLP tasks precision, recall and F1-score [126] are widely used and are defined as as:

$$
\begin{gathered}
\text { Precision }=\frac{T P}{T P * F P} \\
\text { Recall }=\frac{T P}{T P * F N} \\
F 1 \text { score }=\frac{\text { Precision } * \text { Recall }}{\text { Precision }+ \text { Recall }}
\end{gathered}
$$

Recall is a measure of the proportion of all possible target classes identified by the algorithm, where precision is a measure of the proportion of classes identified by the algorithm are true. The F1-score is the mean of precision and recall and is usually reported as the overall accuracy.

NLP algorithms can be compared against multiple annotators by scoring algorithms against the agreement of multiple annotators, as measured by Cohen's Kappa statistic. A Kappa-like statistic was presented by Galton in 1892 as a method of identifying fingerprints using human raters where a match was identified if a certain percentage of raters could agree that a unseen sample matched that of a known fingerprint. Cohen's Kappa statistic was formally introduced in 1960 [127] and is defined as:

$$
\kappa=1-\frac{p_{o}}{p_{e}}
$$

where $p_{o}$ is the probability of an observed class by multiple raters and $p_{e}$ is the random chance of observing all possible classes. $\kappa$ therefore represents the class agreement by multiple raters normalized by the probability of all classes where the class agreement 
is $100 \%$ when $\kappa=1$. Agreement measures such as Cohen's Kappa statistic are employed for inter-annotator agreement for NLP tasks [98]. This is useful to estimate the relevant difficulty of a task in which some tasks may yield low inter-annotator agreement and therefore sets a lower expectation for an algorithm's ability to perform the task.

Human annotation is time consuming and has been listed as one of the major challenges in NLP for healthcare applications. Annotation tools such as BRAT[128] ehost [129] provide a method of rapidly annotating documents in which data files (xml, custom output) store all the annotations in each document. These output files are designed to be read into NLP applications to directly compare annotations picked up by humans annotators and those identified by the algorithm. Thus allows for computation of accuracy measures to be automated and therefore many NLP models can be validated automatically.

\subsubsection{NLP clinical information applications}

There have been extensive studies focussing on creating clinical extraction NLP systems for specific disease areas. The NLP system developed as part of the Linguistic String Project (LSP) was one of the first systems to be used for clinical information extraction [130],[131]. Developed in 1987, a qualitative study first described a system comparing human annotated notes with an automated extraction system in radiology reports, reports and discharge summaries [132]. The Medical Language Extraction and Encoding System (MEDLEE) was developed to detect disease mentions from radiology reports and was scored against physicians' interpretations of 230 radiology reports, achieving $87 \%$ recall and $78 \%$ precision across all disease mentions [133],[134]. Medlee has also been extended to detect breast cancer from mammogram reports, as well as forming the basis of the GENIES that extracts molecular pathways from journal articles [135],[136].

The SymText NLP application [137] was used to detect bacterial pneumonia from chest X-ray scans. The majority vote of 3 physicians was used to manually score 292 X-ray reports from the LDS hospital in Utah and compare the annotations to extracted annotations using SymText. The SymText system uses a syntactical rule-based approach combined with a Naive Bayes Classifier to extract 76 different radiographic findings and 89 different diseases from chest x-ray reports [138]. Pneumonia concepts were split into 4 categories: acute bacterial pneumonia, infiltrate pneumonia, aspiration pneumonia and support pneumonia. Physician average precision and recall for acute bacterial pneumonia compared favourably with that of three physicians and outperformed annotations by lay-persons. 
The i2b2 project used cTAKES and HITex (Health Information Text Extraction) to extract Crohn's disease, Ulcerative Colitis, multiple sclerosis (MS), and Rheumatoid arthritis [139]. A recent study on patients with known MS identified from electronic healthcare records used NLP techniques to accurately extract attributes specific to MS, namely: Expanded Disability Status Scale, Timed 25 Foot Walk, MS subtype and age of onset [140]. A study used clinic letters, available at www.mtsamples.com, to determine whether sentences containing disease and procedure information were attributable to a family member using the BioMedICUS NLP system and variety of phenotype data was extracted from 300 randomly chosen journal titles [141],[142]

There have also been several epilepsy based NLP studies and applications developed. The rule based Epilepsy Data Extraction and Annotation (EpiDEA) system was developed to extract epilepsy information from epilepsy monitoring unit discharge summaries. Categories of information included EEG pattern, past medications and current medication extracted from 104 discharge summaries from Cleveland hospital [143]. The rule-based Phenotype Extraction in Epilepsy (PEEP) pipeline was developed to extract epileptogenic zone, seizure semiology, lateralising sign, interictal and ictal EEG patterns from epilepsy monitoring discharge summaries as Cleveland hospital [144]. A machine based learning NLP pipeline was also developed to identify a rare epilepsy syndrome from discharge summaries and EEG reports [145]

Medication extraction has also been an area of interest for NLP research. The Medication Information Extraction system (MedEx) was developed to extract prescription information, including drug name, dosage, strength and frequency. On a validation set of 50 discharge summaries and 25 clinic notes MedEx was able to achieve an F-score of $93.2 \%$ and $90 \%$ respectively. CPRD prescription data was used to validate 220 prescriptions from anonymised GP records, in which a rule based system was able to achieve $91 \%$ accuracy [146] A rule-based NLP application was developed and applied to the NHS Scotland Prescribing Information System (PIS) [147]. On a validation set of 15,593 prescriptions the system achieved $94.7 \%$ accuracy when extracting full prescription information and was able to generate structured outputs for $92.3 \%$ of $458,227,687$ dosage instructions in the PIS.

There has been a particular focus on mapping extracted terms from NLP applications to existing clinical ontologies. This is particularly important for linking extracted terms to routinely collected data that use ontologies such as SNOMED-CT and ICD-10. A study using 23 citations in the Annals of Internal Medicine and the expert opinion of three physicians described a system that mapped $89 \%$ of terms identified by the physicians to MeSH terms [148]. Various studies have focussed on mapping extracted terms to UMLS concepts for it's ability to map to many other ontologies such 
as SNOMED-CT, READ and ICD-10. A study evaluated the use of UMLS concepts as look up terms for congestive heart failure, chronic obstructive pulmonary disease, acute bacterial pneumonia, neoplasm, pleural effusion without congestive heart failure compared to manually curated lists by clinicians. This study found that using UMLS concepts improved retrieval of terms over than of clinician specified terms[149],[106] and further studies have used a variety of patient information sources such as patient charts, MEDLINE citations and surgical notes [150],[151],[152]. The MetaMap project used UMLS codes to retrieve medical terms from Medline citations and compared the results to the use of NLM MeSH terms. By using UMLS concepts they reported a 14\% improvement on using MeSH terms and is now one of the most widely used algorithms in the NLP community to patient information to UMLS codes. SNOMED-CT terms are widely used in patient records and several studies have proposed methods to map free text to SNOMED-CT. Several studies have used veterans' patient records as a source to validate varying accuracy across a variety of conditions including acute renal failure, venous thromboembolism, pneumonia, myocardial infarction, sepsis $(82 \%, 38 \%, 59 \%, 64 \%$ and $89 \%$ accuracy respectively).

\subsubsection{Genetic Mutation}

Single Nucleotide Polymorphisms (SNPs) are the most prevalent type of genetic mutation in the human genome, with most genes having multiple non-synonymous SNPs and accounting for around 90 percent of genetic variation [153], [154]. SNPs appear in both the coding and non-coding region of the genome in which both have been associated with disease phenotypes. The human genome consists of around 3.2 billion pairs of DNA, with SNPs appearing every 1000-2000 base pairs (between 2-3 million per genome) [155] and on average one person will have 250-300 potentially damaging SNPs that are directly or indirectly associated with disease [156],[157]. SNPs fall into three broad categories.there Nonsynonymous (or missense) SNPs represent a mutation in nucleotide base triplets that cause a change in the amino acid the nucleotides code for. Synonymous SNPs, while containing mutations in nucleotides, do not cause a change in the resulting amino acid. This is due to the fact that there are multiple combinations of nucleotide triplets that code for the same amino acid. Frameshift SNPs involves a deletion or insertion in the amino acid sequence. Multiple insertions and deletions can also occur, but SNPs are estimated to contribute to over $90 \%$ of all known genetic mutation [154] of which $50 \%$ of SNPs have shown to be common ( $20 \%$ of the population have a given SNP) [158].

SNPs can fall anywhere in the genome meaning they are found in regions of DNA that do not code for protein sequences ( $99 \%$ of the human genome) and in coding regions that do code for proteins, known as the exome. Non coding regions have controversially 
been labelled in the past as "junk DNA", where it was assumed that mutations in non-coding regions of the human genome would not contribute to disease, however the ENCODE project showed that over $80 \%$ of non-coding regions serve some purpose such as promotors, enhancers and silencers - all important roles in gene regulation [159]. Despite SNPs in non-coding regions shown to cause diseases such as pancreatic agenesis [160], juvenile idiopathic arthritis [161] and auto-immune conditions [162], the majority of genetic mutation research, especially in non-synonymous SNPs has been focussed on coding regions in the exome as this demographic has been shown to be responsible for around $50 \%$ of human inherited disease [163].

\subsection{Pathogenicity of SNPs}

Pathogenicity in terms of genetic mutation can be described as the ability of a genetic mutation to cause disease. The American College of Medical Genetics and Genomics stated that pathogenic mutations can be determined on two types of evidence: a variant has been previously reported as the cause of disease, or the variant has not been reported as the cause of disease but is expected to be declared as such in future [164]. These categories are broad and problematic for the definition of pathogenicity as illustrated by a study of 402 published severe disease mutations showing that $27 \%$ of these were either common or lacked direct evidence for pathogenicity [165]. Further guidelines such as those proposed by The US National Human Genome Research Institute have suggested 5 categories of mutation associated with causation of disease [166]:

- Pathogenic: contributes mechanistically to disease, but is not necessarily fully penetrant (i.e., may not be sufficient in isolation to cause disease).

- Implicated: possesses evidence consistent with a pathogenic role, with a defined level of confidence.

- Associated: significantly enriched in disease cases compared to matched controls.

- Damaging: alters the normal levels or biochemical function of a gene or gene product.

- Deleterious: reduces the reproductive fitness of carriers, and would thus be targeted by purifying natural selection.

These definitions are far more reflective of that fact that genetic mutation and it's association with disease can range from single-gene, single-mutation causation of disease (high penetrance), such as in cystic fibrosis [167] or Huntington's disease [168] than can be discovered through familial inheritance studies through to common-disease, 
common-variant associations where multiple mutations common in the population sum to causative effects (low penetrance) such as Alzheimer's disease [169] that require large-scale GWAS studies to identify disease risk. The ClinVar dataset curates a record of all variants that can be defined as pathogenic, likely pathogenic, likely benign, benign and uncertain following review of aggregated submissions and publications [170]. Other databases such as dbSNP [171] and Uniprot/Swissprot also record pathogenicity/benign status where the entry point in terms of association to disease meets at least the minimum requirement as set out previously in [166], that is, a variant deemed as pathogenic at least contributes to a disease phenotype.

\subsection{Whole genome sequencing and the need for SNP prediction paradigms}

Frederick Sanger introduced dideoxynucleotide sequencing, or Sanger sequencing of DNA [172] were manual techniques harnessed to sequence individual genes and cells. More sophisticated techniques such as shotgun sequencing were introduced in which bacterial genomes such the influenza genome (2 million DNA base pairs) was able to be sequenced [173], with the first eukaryote genomes, a strain of yeast - Saccharomyces cerevisiae ( 12 million base pairs) and a type of worm - nematode elegans (100 million base pairs) were sequenced [174],[175]. Next Generation Sequencing technologies were developed to allow high-throughput sequencing of large genomes such as the common fruitfly - Drosophila melanogaster (135 million base pairs) [176] and finally in 2001 the human genome (3 billion base pairs), costing an estimated $£ 750$ million US dollars [177]. The cost of whole genome sequencing has vastly decreased since Craig J Venter sequenced his genome in 2001 as part of the Human Genome Project from $£ 750$ million US dollars to $£ 1000$ US dollars today. This remarkable decrease in cost is owed to advances in sequencing technology over the past 15 years, and to highlight this, Figure 1.4 shows the decrease in whole genome sequencing when compared to what the decrease might be if following Moore's Law. 


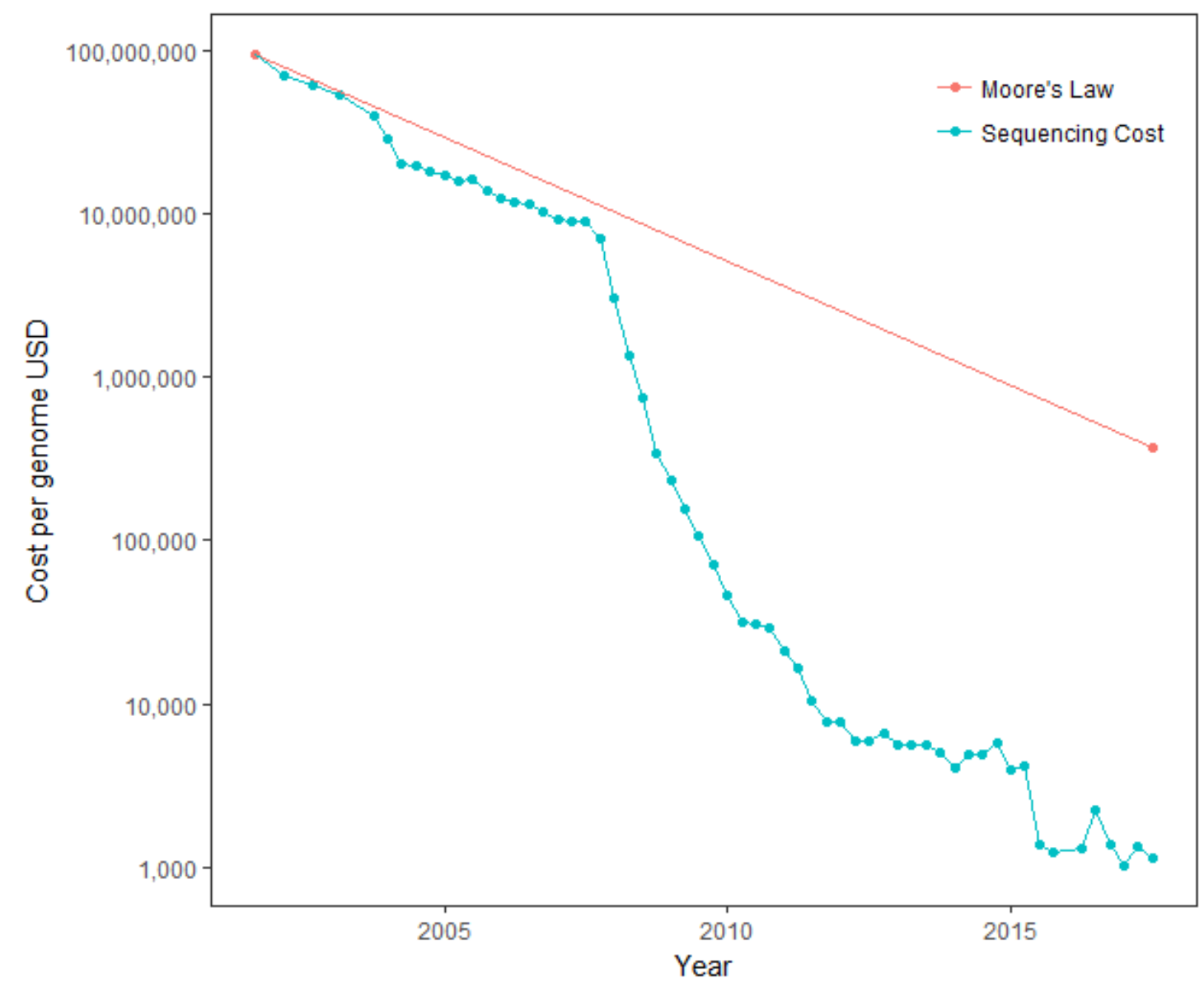

Figure 1.4: Decrease in whole genome sequencing since the Human Genome Project when compared to the expected rate of decrease following Moore's Law

The relatively low cost of whole genome sequencing has enabled whole genome / exome cohort projects such as The 1000 Genomes Project [178], The 100,000 Genomes Project [179], UK Biobank [180], ExAC [181] and aggregation services such as the Genome Aggregation Database (gnomAD) that contains 15,496 genomes and 123,136 exomes for unrelated individuals. The number of variants processed from whole genome sequencing outstrips the ability for genetic research to comprehensively study each and every variant through traditional laboratory techniques [182]. Typically whole genome sequencing one person will generate 3,000,000 variants and whole exome sequencing will generate 30-50,000 variants and it is therefore vital to find ways of focussing on variants likely to impact disease in advance of more thorough analyses [183]. 


\subsection{Common features used in prediction of pathogenicity}

There are a variety of hypotheses that help when considering the pathogencity of SNPs. A study of 561 disease causing SNPs and from the SWISSPROT database that contained details of 2D and 3D structure found that over 70\% of these SNPs were found in structurally important regions such as binding sites and sites with low solvent accessibility [184]. The allele frequency on non-synonymous SNPs that caused disease was found to be lower than that of non-synonymous SNPs, indicating that nature selects against pathogenic SNPs and that disease causing SNPs may be found at sites that are conservative [153]. The BLOSUM62 matrix was developed to produce a system that scored all possible 210 amino acid substitution of the 20 standard amino acids based on an alignment of 500 protein sequences (BLOSUM62 matrix shown in Figure 1.5).

$$
\begin{aligned}
& \text { Ala } 4 \\
& \text { Arg }-1 \quad 5 \\
& \text { Asn } \quad \begin{array}{llll}
-2 & 0 & 6
\end{array} \\
& \text { Asp } \begin{array}{lllll}
-2 & -2 & 1 & 6
\end{array} \\
& \begin{array}{llllll}
\text { Cys } & 0 & -3 & -3 & -3 & 9
\end{array} \\
& \text { Gln } \begin{array}{lllllll}
-1 & 1 & 0 & 0 & -3 & 5
\end{array} \\
& \text { Glu } \begin{array}{llllllll}
-1 & 0 & 0 & 2 & -4 & 2 & 5
\end{array} \\
& \text { Gly } \quad \begin{array}{llllllll}
0 & -2 & 0 & -1 & -3 & -2 & -2 & 6
\end{array} \\
& \text { His } \begin{array}{lllllllll}
-2 & 0 & 1 & -1 & -3 & 0 & 0 & -2 & 8
\end{array} \\
& \text { Ile } \begin{array}{lllllllllll}
-1 & -3 & -3 & -3 & -1 & -3 & -3 & -4 & -3 & 4
\end{array}
\end{aligned}
$$

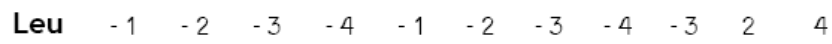

$$
\begin{aligned}
& \begin{array}{lllllllllllll}
\text { Lys } & -1 & 2 & 0 & -1 & -3 & 1 & 1 & -2 & -1 & -3 & -2 & 5
\end{array}
\end{aligned}
$$

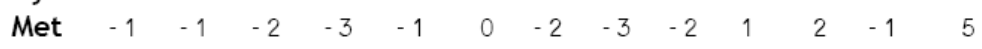

$$
\begin{aligned}
& \text { Phe } \begin{array}{llllllllllllll}
-2 & -3 & -3 & -3 & -2 & -3 & -3 & -3 & -1 & 0 & 0 & -3 & 0 & 6
\end{array} \\
& \begin{array}{llllllllllllllll}
\text { Pro } & -1 & -2 & -2 & -1 & -3 & -1 & -1 & -2 & -2 & -3 & -3 & -1 & -2 & -4 & 7
\end{array} \\
& \begin{array}{lllllllllllllllll}
\text { Ser } & 1 & -1 & 1 & 0 & -1 & 0 & 0 & 0 & -1 & -2 & -2 & 0 & -1 & -2 & -1 & 4
\end{array}
\end{aligned}
$$

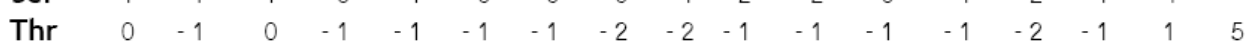

$$
\begin{aligned}
& \begin{array}{lllllllllllllllllll}
\text { Trp } & -3 & -3 & -4 & -4 & -2 & -2 & -3 & -2 & -2 & -3 & -2 & -3 & -1 & 1 & -4 & -3 & -2 & 11
\end{array} \\
& \begin{array}{llllllllllllllllllll}
\text { Tyr } & -2 & -2 & -2 & -3 & -2 & -1 & -2 & -3 & 2 & -1 & -1 & -2 & -1 & 3 & -3 & -2 & -2 & 2 & 7
\end{array} \\
& \text { Val } \quad \begin{array}{llllllllllllllllllll}
0 & -3 & -3 & -3 & -1 & -2 & -2 & -3 & -3 & 3 & 1 & -2 & 1 & -1 & -2 & -2 & 0 & -3 & -1 & 4
\end{array} \\
& \text { Ala Arg Asn Asp Cys Gln Glu Gly His lle Leu Lys Met Phe Pro Ser Thr Trp Tyr Val }
\end{aligned}
$$

Figure 1.5: The BLOSUM 62 matrix where higher scores indicate higher frequency of substitution. Each amino acid substitution is scored in accordance to it's frequency, where lower frequency substitutions are said to be conserved and potentially selected against by natural selection.

On average 5 of the possible 19 substitutions for a given amino acid has found to be non-conservative and is a potential predictor for SNPs associated with disease [153]. A study comparing the distributions of 1169 disease associated non-synonymous SNPs and 741 neutral SNPs found a significant difference when plotted against BLOSUM62 scores inferring that lower frequency substitutions are associated with pathogenicity [185]. The Sifting Intolerant From Tolerant predictor[186],[187] was 
developed from the hypothesis that position specific information of where a SNP is found might be a predictor of disease/neutral status given that the plasticity of proteins can change across sequences [188], [189],[190]. To test this, the SIFT study took datasets that collected variants found in the Laci, HIV-1 and T4 lysozyme proteins [191],[192],[193],[194] and performed a multiple sequence alignment to other proteins in their respective families using PSI-BLAST [195] and compared disease/non-disease SNPs in these proteins to the position of the multiple sequence alignment. The results showed that the accuracy of SIFT was $66 \%, 86 \%$ and $45 \%$ for the SNPs in each protein respectively and that using position specific information from multiple sequence alignment was able to correctly identify the disease status of $14 \%$ more SNPs overall when compared to using BLOSUM62 scores. The study showed that position specific information was however more important in some proteins than others, in particular the low prediction accuracy of the T4 lysozyme proteins.

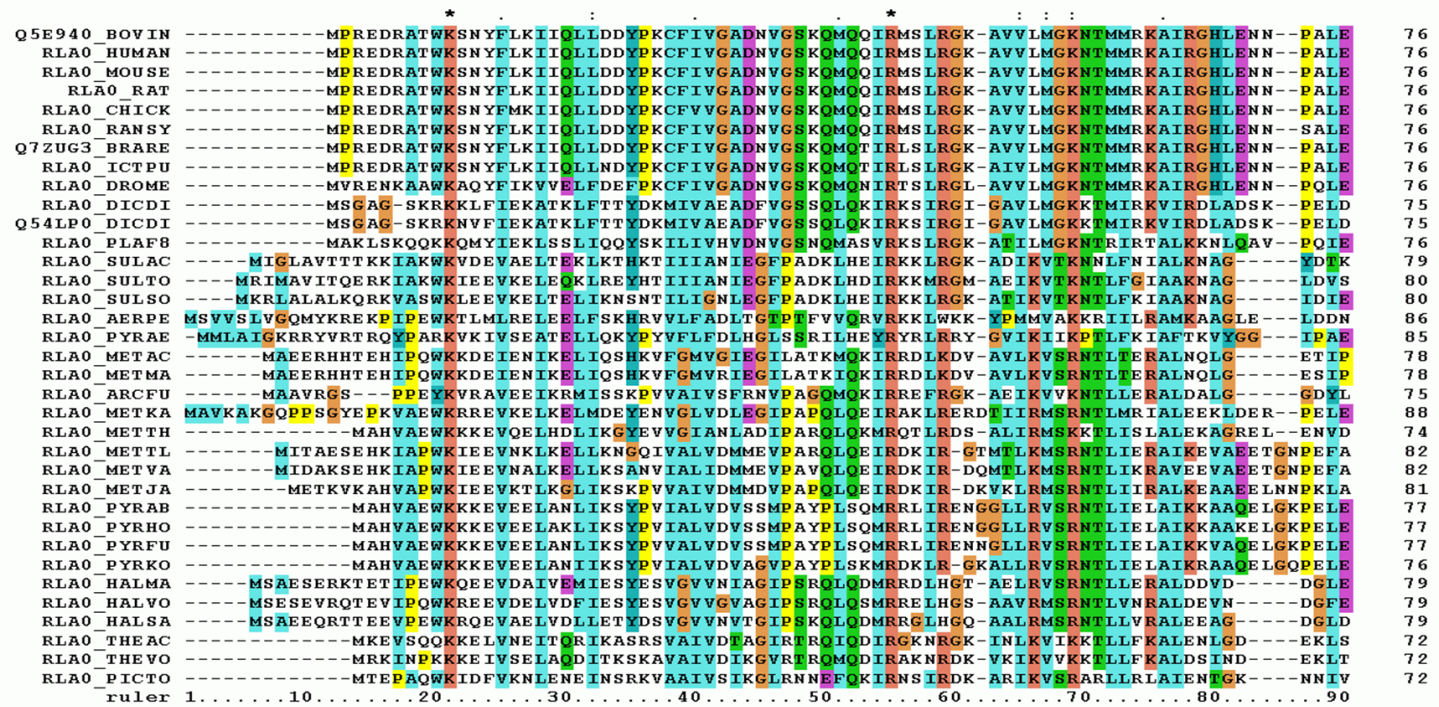

Figure 1.6: A multiple sequence alignment of transmembrane proteins from different species. Conserved regions are in red where alignment of different proteins shows no difference in amino acids across all proteins in this position. Conserved regions are therefore hypothesized not to tolerate geneitc variation and are deemed hotspots for pathogenic mutations.

The PolyPhen-2 prediction server was developed with a range of features that included multiple sequence alignments and molecular function information [196]. Prominent features used by PolyPhen was difference position specific scores for both wild type and mutant based on multiple sequences alignments [197], predictions of functional region such as transmembranes [198], coils [199] and peptide signals [200] as well as known 2D and 3D structure from DSSP and PDB [201],[202]. The study used a Naive Bayesian classifier, a type of machine learning classifier, to take these features as input and classify into three categories: benign, possibly damaging and probably damaging. 
The HumDiv (3,155 damaging and 6,231 benign SNPs) and HumVar (13,032 damaging and 8,946 benign SNPs) datasets, datasets annotated with damaging/benign status from Uniprot, were used for training/testing of the Naive Bayes classifier using a 5 -fold cross validation technique where training and testing sub-sets were randomly sampled. The PolyPhen method was able to achieve $93 \%$ and $72 \%$ true positive rates for damaging SNP detection using a $20 \%$ false positive rate on the HumVar and HumDiv datsets respectively [203].

\subsubsection{Machine learning classification for SNP pathogenicity}

Machine learning classification is a staple of SNP prediction techniques. They are used effectively when a large number of SNPs in both benign and pathogenic classes are used to train a model with features hypothesized to be useful in discriminating between pathogenic and benign SNPs. Machine learning techniques use the interaction of every feature against all other features to make a prediction, meaning that even features not thought to have a strong influence on classification may be useful to increase accuracy by a small amount overall, or increase accuracy substantially in subsets of samples that do not adhere to common hypotheses such as position specific sequence information or structural features.

The PolyPhen server uses a Naive Bayes classifier, but many other machine learning techniques have been used in SNP prediction as shown in Table 1.7.

Table 1.7: Commonly used SNP prediction programs that utilize machine learning

\begin{tabular}{lll}
\hline Algorithm & Citation & Machine Learning Classifier \\
\hline DANN & {$[204]$} & Convolutional Neural Network \\
MetaLR & {$[205]$} & Logistic regression \\
MutationAssessor & {$[206]$} & SNP prediction application \\
REVEL & {$[207]$} & Random Forest \\
FATHMM & {$[208]$} & Hidden Markov Model \\
PolyPhen & {$[196]$} & Nä̈ve Bayes \\
CADD & {$[209]$} & Support Vector Machine \\
MetaSVM & {$[205]$} & Support Vector Machine \\
VEST3 & {$[210]$} & Random Forest \\
PHD-SNP & {$[211]$} & Gradient Boosting Learner \\
PolyPhred & {$[203]$} & Decision Tree \\
\hline
\end{tabular}




\subsubsection{SNP datasets}

A number of databases provide pathogenic/benign status. There are many public databases that record SNPs from population studies such as the National Center for Biotechnology Information (NCBI) SNP database, dbSNP [171], ClinVar [212] and the Human Genome Variation database, HGVBase [213]. Other databases focus on curating SNPs known to be associated with causing disease (rather than pathogenicity) such as the Online Mendelian Inheritance in Man (OMIM) [214], the Genetic Association Database (GAD) [215] and The Human Gene Mutation Database (HGMD) [216]. One of the most widely used datasets along with HGMD (FATHMM, REVEL, CADD, DANN) is the humvar dataset https://www.uniprot.org/docs /humsavar curated by Uniprot, which as of June 2018 contains over 70,000 SNPs with known status and has been used to train classifiers such as PolyPhen, VEST3, MetalR, MetaSVM and PHD-SNP.

Currently the REVEL classifier has the highest published accuracy when compared with other existing scores in two independent test sets, Clinvar and SWISSVAR, achieving AUC measures of 0.83 and 0.95 respectively. REVEL is perhaps unique in that it only uses a relatively small set of 13 features, none of which are derived from protein features such as secondary structure or multiple sequence alignments, but instead it uses the output of 13 SNPs prediction classifiers and combines the results using a Random Forest classifier [207]

\subsubsection{Bioinformatics software and annotation programs to obtain SNP features}

As well as obtaining SNPs with known pathogenicity status, it is important for machine learning classifiers to obtain many features such as multiple sequence alignments and region annotation. There are various bioinformatics pipelines that can help source these features. There exists many multiple sequence alignment programs that can be used to obtain conservation measures at each position of a sequence. These include BLAST [217], PSIBLAST [195], MUSCLE [218], CLUSTAL [219], UBLAST [220] and HMMER [221]. In addition to multiple sequence aligners for conserved regions of sequences, there are various tools exist such as GERP (Genome Evolutionary Rate Profiling) and phyloP [222], phastcons [223] and SiPHy (Site Specific Phylogenetic analysis) [224] that compute conservation as a function of evolutionary selection over all positions of protein sequences.

Region annotation is useful to detect if a SNP falls with certain functional regions such as transmembranes or binding sites. While databases such as DSSP and PDB 
exist that catalogue such features, there are many programs that compute or predict such features. The PSIPRED server can be used to predict if positions in an amino acid sequence are likely to be in a coil, helix or sheet category of secondary structure [225], and various programs can predict functional regions such as binding sites [226], transmembrane regions [198] and protein-protein interaction sites [227].

There are also annotation aggregation services that take a SNP as input (usually rsID, BED or HGVS format) and query large pre-computed databases to gather a vast range of features that include multiple sequence alignments, regional annotations, scores from many prediction software (dbNFSP [228]) and allele frequency measures computed as part of 1000 genomes, exAC and gnomAD. Three commonly used aggregation tools are Variant Effect Predictor [229], snpEFF [230] and Annovar [231]. Such tools are available as web services and standalone programs and particularly useful in SNP annotation because their databases storing many SNP features are kept up to date, making them a one-stop shop for SNP annotation.

\subsection{Chapter Summary}

This thesis is summarised as follows:

- Epilepsy is prevalent in $1 \%$ of the population and can be caused by inherited genetic mutation of acquired through lesions in the brain due to injury. Epilepsy can be presented in many sub-types and seizure types of which many different types are attributed to inherited genetic mutation in certain genes. Severity of epilepsy can range in terms of seizure frequency, and various treatment regimes using anti-consultants exist to control seizures

- The burden of epilepsy can be measured in other ways than seizure frequency. Patients living with epilepsy are known to have lower socio-economic status and those with poorly controlled epilepsy perform poorer in education than their peers.

- Epilepsy research in epidemiology is restricted to using routinely collected healthcare records that often contain rich data desired for impactful studies in epilepsy. Large data banks such as the SAIL Databank can provide data linkage across multiple health and social care datasets, however this still does not solve the problem of data shortage for people with epilepsy

- Natural Language Processing is an emerging field in healthcare that has benefited from machine learning and the explosion of big datasets. Natural Language Processing offers the potential to collect data form clinic letters that does not 
end up in routinely collected datasets.

- The emergence of Next Generation Sequencing has enabled genetic research to be conducted on a much larger scale and help with genetic discoveries. The sheer volume of data recorded poses a problem in how to make sense of millions of variant data per individual. Consequently prediction paradigms are necessary to prioritise which variants require thorough genetic analysis to make genetic research cost effective.

- Various prediction techniques have been developed with the aid of machine learning and adopted for genetic research. Such programs exist as part of larger pipelines that filter pathogenic variants from benign variants, however many lack the specificity to prevent large amounts of benign variants being needlessly analysed in molecular assays.

- Linked data of routinely collected data, clinic letters and genetics will play an important role in the future of healthcare research, and efforts must be made to facilitate the linkage of emerging data types at scale.

\subsection{Summary of aims and objectives}

A summary of the aims and objectives are:

1. Using routinely collected healthcare data stored in the SAIL databank to identify persons with epilepsy

2. Link primary care records of persons with epilepsy to other datasets within the SAIL databank to study societal burden of epilepsy and the effects of antiepileptic drugs

3. Explore the possibilities of incorporating clinical free text into existing patient records by using NLP techniques. Target information will consist of data that is difficult to obtain or non-existent within the SAIL databank

4. Investigate various methods of determining or predicting pathogenicty of SNPs, in particular to study SNPs found within epilepsy associated genes.

5. Create an algorithm that accurately predicts pathogenicity for missense SNPs in epilepsy to aid SNP prioritisation for downstream structure/functional analysis 


\section{Chapter 2}

\section{Methods}

The following chapter describes the methods and materials used to mine health trends in data from the SAIL databank, extract information from unstructured clinic letters using NLP building a pipeline for assessing the impact of SNPs, with a focus on how the outputs of NLP and SNP analysis could potentially enrich data audited from routinely collected healthcare records. The SAIL databank holds a large all-Wales database of routinely collected electronic healthcare records that can be potentially be enriched by linking bespoke datasets, such as clinic letters (unstructured text) and whole genome/exome (NGS) data. Patient records at the SAIL databank anonymous - specifically by use of an encrypted NHS number, so that these records can be linked anonymously across different datasets i.e. GP records and hospital admissions. The NLP methods described in this chapter and chapter 4 are intended to be used to extract information from clinic letters using an automated computer algorithm in which the results could be linked to the SAIL databank and provide rich patient information that doesn't exist in SAIL. Similarly, the SNP pipeline described in this chapter and chapter 5 is designed to take in SNP information, filter it on various criteria and output the data in a format that can be linked to patient data in the SAIL Databank. Much of the code underpinning the methods is written in SQL, R, perl, bash for calling open source bioinformatics programs, as well as an array of UNIX/GNU programs such as sed and awk. Complete code is found at www.github.com/https://github.com/arronlacey/Epilepsy-GATE-app (Chapter 3).

\subsection{The SAIL Databank}

The SAIL databank holds many routinely collected electronic healthcare databases, where a person's records can be identified and linked between datasets by an encrypted 
NHS number called an ALF (Anonymous Linking Field). Figure 2.1 shows the anonymisation and linkage process that ensure SAIL uses encrypted NHS numbers or Anonymous Linking Fields (ALFs) to perform linkage.

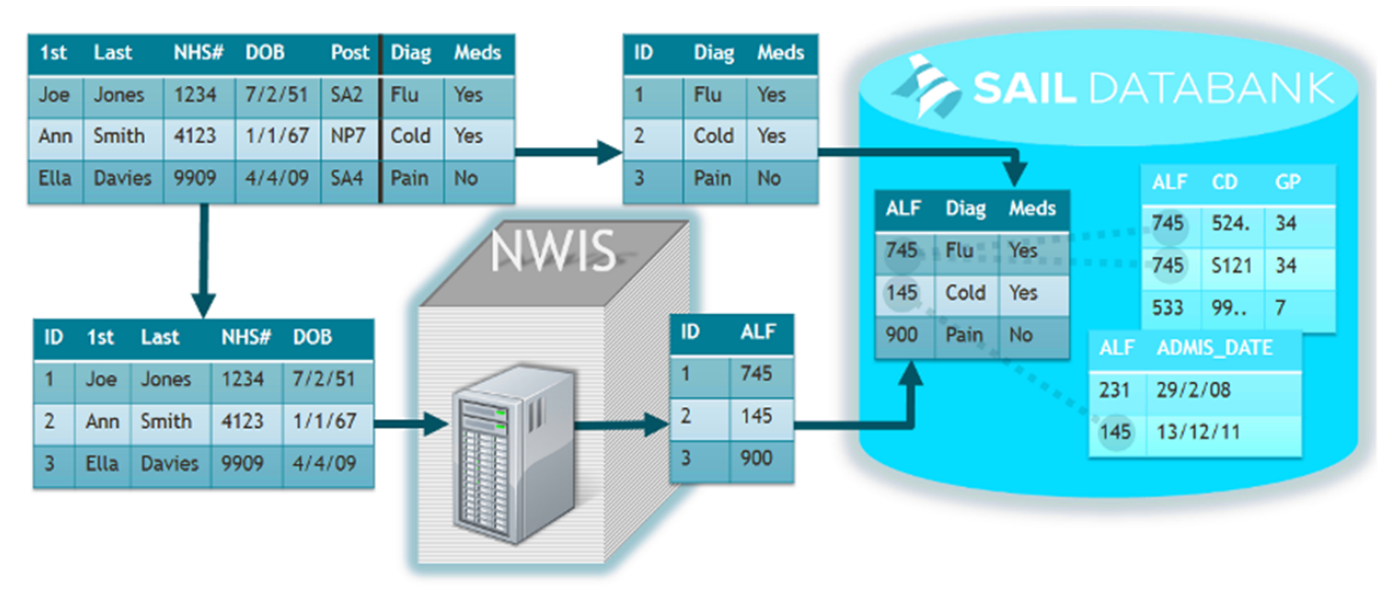

Figure 2.1: SAIL Databank split file procedure. Data is split at source into identifiable data and clinic data. The identifiable data sent to The NHS Wales Information Service where each NHS number is encrypted before being sent to the SAIL Databank. The clinic data is sent directly to the SAIL Databank, and is joined to the encrypted identifiable data by an internal system ID that is present in both datasets.

\subsubsection{Ethics and Governance}

All proposed SAIL projects undergo a review process by an IGRP (Information Governance Review Panel) for approval. Researchers must complete an IGRP form that details the scope of the project and what datasets are required. Approved projects must be deemed feasible and present no risk to patient identification. While all NHS numbers are encrypted in SAIL, it may be possible for Clinicians, without taking data out of the SAIL databank to identify a patient with a rare combination of information in their medical records e.g. a 35 year old man living in the ABMU health board area that has Becker's muscular dystropy, focal seizures with secondary generalisation and being prescribed multiple anti-epileptic drugs for multiple seizures per day. SAIL does not allow individual level data to be taken out of the gateway and must be summarized so that it conforms to small number disclosure rules of never reporting on groups with less than 5 persons. Data must be requested out and reviewed by the SAIL Analyst Team to enforce these rules.

\subsubsection{Assessing the burden of disease using the SAIL Databank}

The work of this thesis focuses largely on how to identify disease phenotypes, their effects on quality of life and how to treat and manage the disease. The Wales 
Epilepsy Research Network (WERN) at Swansea University has conducted world class research into epilepsy genetics, and has both clinical and research expertise in the field of epilepsy. The SAIL databank presents an ideal opportunity to further our understanding of the burden of epilepsy in health care. Where exome analysis can identify the exact cause and mechanisms of epilepsy, patient records within SAIL can answer questions on social outcomes, health utilisation and drug efficacy for patients with epilepsy. Being a member of WERN and integrating into the wide variety of researchers has helped form novel and important questions for this thesis.

\subsubsection{Forming Research Questions}

In 2008 a group survey conducted on behalf of the DUETs (Database of Uncertainty about the Effects of Treatments) and James Lind Alliance brought together patients, clinicians, patient carers and researchers to address questions regarding treatment of conditions, including epilepsy www.library.nhs.uk/duets. In addition, all the Neurology Consultants across Wales were asked to contribute research questions that SAIL may or could answer. Many of the items highlighted form the research basis for this thesis involving SAIL data, with an emphasis on:

1. Research into better treatments and seizure control

2. Research into ensuring current treatments are as effective as possible

3. Research into stigma associated with epilepsy

4. Research into epilepsy and other medical conditions

5. Research into patient information

These questions set many challenges when using data in the SAIL databank and were considered to prioritise the studies described in chapter 3 of this thesis. The first problem is to correctly identify epilepsy phenotypes within routinely collected primary care data, and the second is to obtain treatment and social status of those with epilepsy. A scoping exercise was carried out to identify datasets that would contain the required data build an epilepsy patient profile within SAIL data. Four core datasets were identified within the SAIL databank to obtain epilepsy metrics General Practice dataset, Secondary care dataset (PEDW), the Welsh Demographic Service dataset and the Office of National Statistics (ONS) Deaths dataset. 


\subsection{SAIL Datasets}

The following section describes the core datasets in SAIL, including those used in this thesis.

\subsubsection{GP dataset}

Each GP practice in Wales uses a clinical information system to capture patient records. The Primary Care IM\& T Programme developed a piece of software called Audit+ that is provided free of charge to all Welsh GPs. It was designed to facilitate the capture and transfer of GP data to external sources. A SAIL module is built into Audit+ that automatically two data extracts: file 1 contains demographic data and file 2 contains clinical data, where an internal system ID is shared between both files. Both files are securely transferred to SAIL and are anonymised via the encryption process outlined in [61]. Each GP practice in Wales has been invited to participate, where only those consenting have their data transferred into SAIL. Over length of study of this thesis, SAIL GP participation has increased from $40 \%$ of Welsh GP practices to $75 \%$.

The dataset contains two unique identifiers: patient ID (encrypted NHS Number) and GP ID (also encrypted). Demographic data available includes week of birth, sex and dates of the beginning/end of registration with a given GP practice. Clinical information includes reason for attendance via version 2 READ codes, date of attendance and any laboratory result such as blood pressure. There are over 300,000 READ codes that can be used to define diseases, drug prescriptions, symptoms, referrals to specialists and laboratory results. A subset of READ codes were used to define:

1. Epilepsy diagnosis

2. Anti-epileptic drugs (AEDs)

Using a combination of these two categories, a diagnosis of epilepsy in the SAIL dataset is defined as a patient that has a diagnosis code for epilepsy followed by a repeat anti-epileptic drug prescription within 6 months. A repeat AED prescription is used as a pre-cautionary measure to prevent suspected, unconfirmed epilepsies being used in any epilepsy cohorts generated. The reasoning behind this is that epilepsy and treatment plans are confirmed by a specialist, not in a GP setting. It is possible a GP may use a diagnosis code for epilepsy to denote suspected epilepsy as well as a code to refer to a specialist. The repeat prescription is indicative of that patient being seen by a specialist and prescribed a AEDs beyond a potential trial period. 
Defining epilepsy in the GP dataset held within SAIL is useful because it can be used to build up a patient profile of epilepsy at first point of health care in Wales, as well as link to other datasets.

\subsubsection{Secondary Care dataset}

The SAIL databank receives an annual extract of all Welsh inpatient hospital data. This dataset is processed using the same split file procedure where the National Wales Information System (NWIS) acts as a TTP and processes the extract useable within SAIL called PEDW. PEDW contains all hospital admissions in Wales from 1998 and uses the ICD-10 coding system to record admissions to inpatients. Each hospital employs teams of clinical coders to records the reason for admission as determined from consultation of doctors' notes, as well as any operations and costs associated with the admission. Other variables included within PEDW are date of admission, length of stay and consultation speciality. Date recorded in PEDW is designed to reflect the care pathway of an admission to secondary care, and with careful use of ICD-10 code selection, it is possible to identify patients that are admitted to secondary care and the reason for being admitted. In particular there are ICD-10 codes for seizures and status epilepticus, as well as other common outcomes associated with epilepsy such as seizures and stroke.

\subsubsection{Welsh Demographic Service}

The Welsh Demographic Service (WDS) was introduced in 2009 to manage administrative and demographic data for NHS patients in Wales. It contains address information as recorded by a patients' GP, which is mapped to Lower Super Output Area (LSOA), Middle Super Output (MSOA) and Local Health Board (LHB) by the address postcode. These are geographical units with LSOA's accounting for between 5-10 postcodes and can be mapped to The Welsh Index of Multiple Deprivation (WIMD) to measure social deprivation. The Welsh Government's official measure of deprivation is the Welsh Index of Multiple Deprivation (WIMD) http://wales.gov.uk/topics/statistics/theme/wimd/?lang=en and is readily available in the SAIL databank for any person registered with a Welsh GP. It comprises of 8 domains:

1. Income

2. Employment

3. Health

4. Education 
5. Access to Services

6. Community Safety

7. Physical Environment

8. Housing

where a deprivation score can be obtained for each domain or combined into one score. The WIMD is therefore one way of measuring social deprivation to some degree in those living with epilepsy. An explanation of how the WIMD score is calculated is given in Figure 2.2.

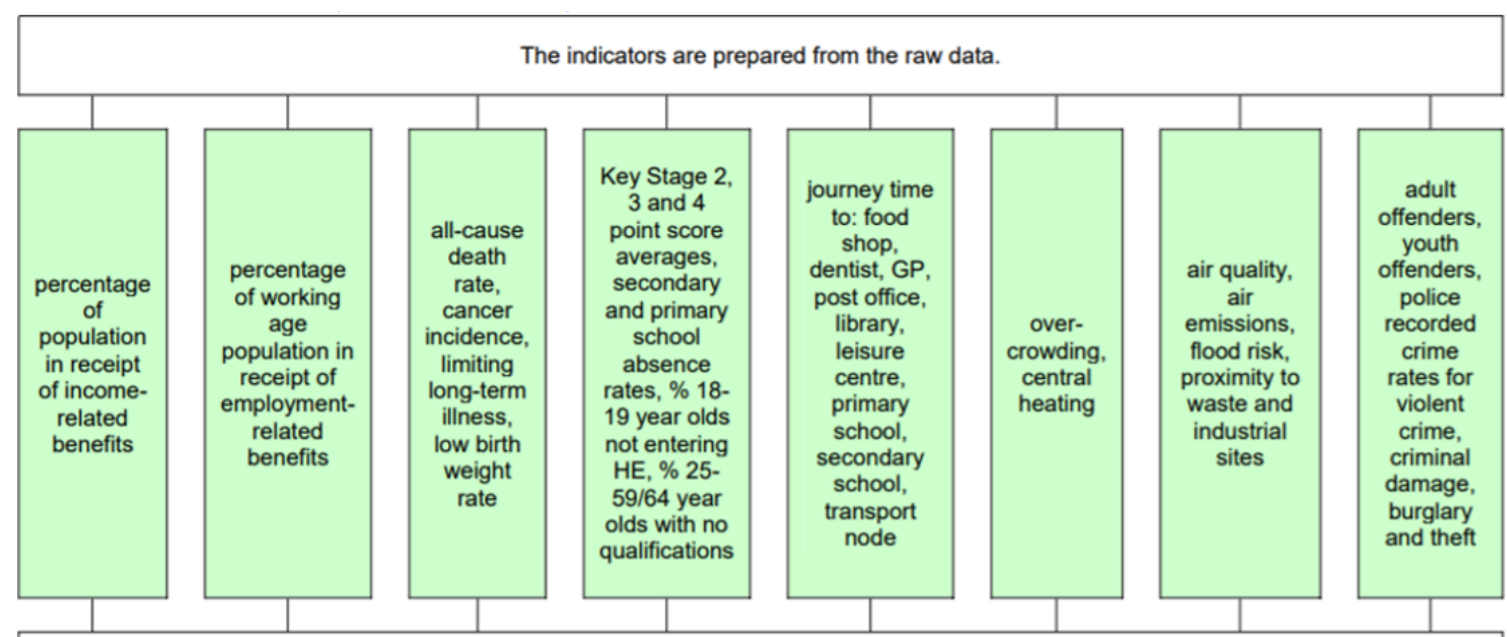

Each indicator is transformed to a normal distribution, and the normal scores are weighted and added to give the domain score. Weights are generated by factor analysis where there are more than three indicators. Where there are three or fewer indicators, factor analysis is not possible, and equal weights have been assigned. Note that the physical environment domain has three indicators, as the air quality and air emissions indicators form a single sub-domain

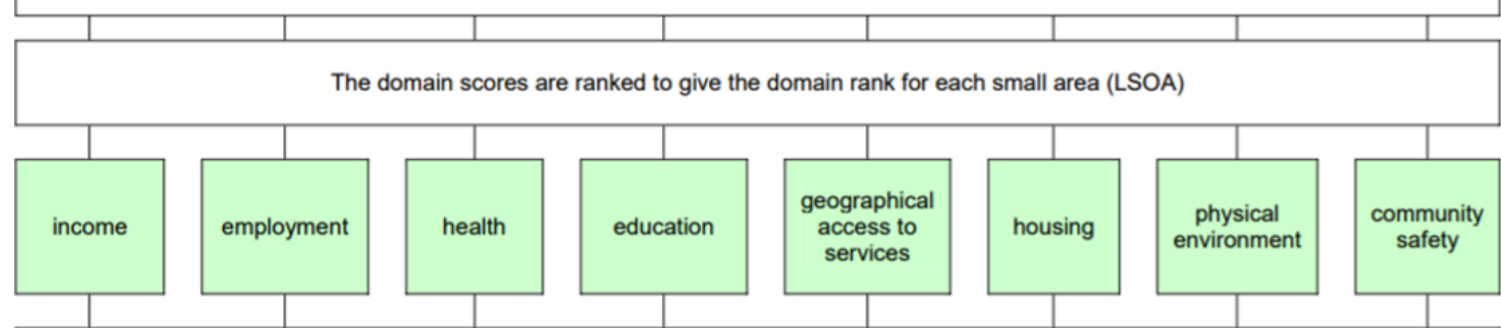

The domain ranks are exponentially transformed, and added together, weighted by the weights given below, to produce the WIMD score

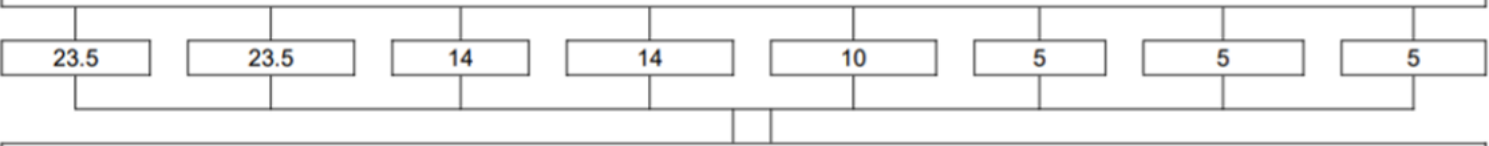

The WIMD scores are ranked

WIMD rank for each small area (LSOA)

Figure 2.2: Flow chart of how the WIMD score is calculated from 8 different domains. Taken from http://webarchive.nationalarchives.gov.uk/20150505155421/http://gov.wa les/docs/statistics/2011/110831wimd11summaryen.pdf 


\subsubsection{ONS deaths}

SAIL contains both annual and monthly extracts from the ONS database. This dataset contains date of death, primary and secondary causes of death, location of death (LSOA), place of death (hospital,home etc) and age of death. ONS deaths contains all deaths in Wales from 2003 onwards. Causes of mortality are not well understood in epilepsy related deaths, and death certificate information alone can only provide so much evidence. For example Sudden Unexpected Death in Epilepsy (SUDEP), an uncommon outcome of epilepsy rarely gets recorded in death certificates due to the cause of death not being clear, and so the amount of people dying from SUDEP is thought to be underestimated in the epilepsy population. In some cases it is thought to be preventable, however the risk factors are not well understood and there is currently no genetic explanation for SUDEP, and as such a research priority in epilepsy. The work in this thesis hypothesizes that linking death certificate information from ONS into GP and secondary care records can provide further insights into risk factors associated with SUDEP.

\subsection{Data Linkage}

\subsubsection{Structured Query Language}

SQL (Structured Query Language) queries use set theory to join multiple datasets together and aggregate individual records into groupings for statistical analysis. Unique identifiers are present in all datasets within SAIL, this is usually the encrypted NHS number of a patient called an Anonymous Linking Field, ALF. For example, consider the two tables and SQL code used to join them by the ALF:

\begin{tabular}{|c|c|c|c|c|c|}
\hline \#\# & ALF_E & GNDR_CD & DRUG & GP_DATE & GP_PRACTICE \\
\hline \#\# & 120000001 & M & LTG & 2001-01-01 & SW1 \\
\hline \#\# & 220000001 & M & VPA & 2001-04-02 & \\
\hline \#\# & 320000002 & $\mathrm{~F}$ & CBZ & 2001-04-10 & \\
\hline \#\# & 420000003 & M & CBZ & 2001-11-04 & NEA \\
\hline \#\# & 520000004 & $\mathrm{~F}$ & VPA & 2001-01-31 & CAF4 \\
\hline \#\# & 620000005 & $\mathrm{~F}$ & LTG & 1999-04-06 & POW2 \\
\hline \#\# & \multicolumn{5}{|c|}{ ALF_E HOSP_DATE HOSP_ID } \\
\hline \#\# & 120000001 & \multicolumn{2}{|c|}{$2001-05-10$} & $7 \mathrm{AE}$ & \\
\hline \#\# & 220000003 & \multicolumn{2}{|c|}{$2001-11-11$} & ONA & \\
\hline \#\# & 320000004 & \multicolumn{2}{|c|}{$2001-02-14$} & 9DN & \\
\hline \#\# & 420000005 & \multicolumn{2}{|c|}{$2007-03-04$} & G4K & \\
\hline \#\# & 520000006 & \multicolumn{2}{|c|}{$2009-03-15$} & $7 \mathrm{AE}$ & \\
\hline
\end{tabular}




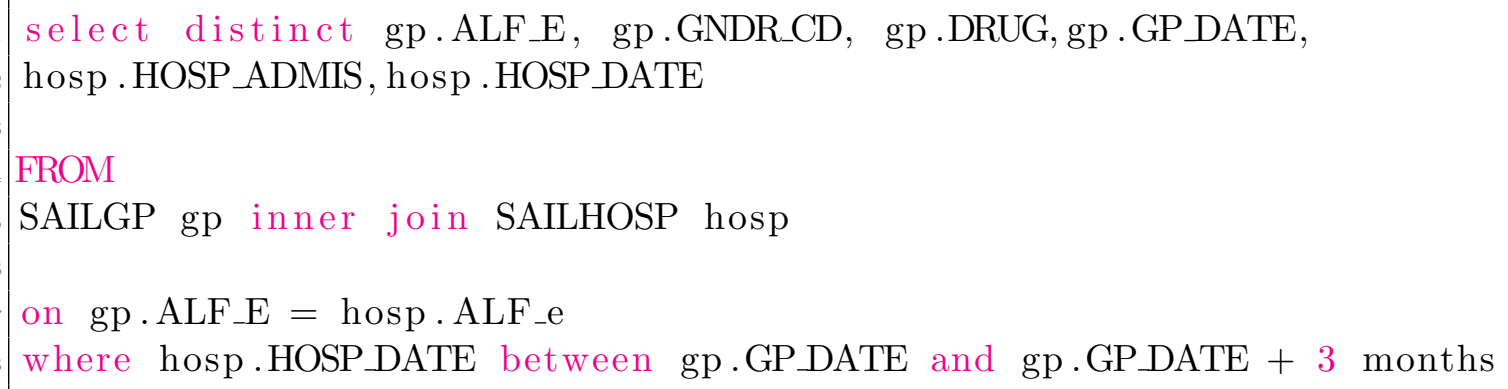

Figure 2.3: A simple SQL script to return encrypted patient identifiers along with the gender and date of birth of the patient where the patient must be female and born after the 1st of January 1990.

The first table is GP data containing prescriptions for anti-epileptic drugs, and the second table is hospital admissions for seizures. It is possible to find persons who have been admitted to hospital for a seizure within 3 months of a drug prescription. The code in figure 2.3 demonstrates how to join the GP data and hospital data together, and the results are shown in figure 2.3.1.

\begin{tabular}{|c|c|c|c|c|c|c|}
\hline \#\# & ALF_E & GNDR_CD & DRUG & GP_DATE & HOSP_ADMIS & HOSP_DATE \\
\hline \#\# & 120000001 & M & LTG & $2001-01-01$ & N & NULL \\
\hline \#\# & 220000001 & M & VPA & $2001-04-02$ & Y & $2001-05-10$ \\
\hline \#\# & 320000002 & $\mathrm{~F}$ & $\mathrm{CBZ}$ & $2001-04-10$ & $\mathrm{~N}$ & NULL \\
\hline \#\# & 420000003 & M & $\mathrm{CBZ}$ & $2001-11-04$ & Y & $2001-11-11$ \\
\hline \#\# & 520000004 & $\mathrm{~F}$ & VPA & $2001-01-31$ & $\mathrm{Y}$ & $2001-02-14$ \\
\hline
\end{tabular}

Once a basic data linkage is established amongst SAIL datasets the aim is to filter the linked datasets down to a "final" dataset ready for statistical analysis.

\subsubsection{Quality checking routinely collected data}

It is important to note that routine healthcare datasets were not designed for research purposes, rather collected at point of care for costing purposes or to supplement decision making processes. With this in mind, any routinely collected healthcare dataset requires careful consideration of how to interpret the data and understand the limitations. The majority of the data is entered manually by trained professionals, but there is still the potential for human error in data entry in which it is impossible for some error to be rectified by retrospectively cleaning the data.

Data cleansing refers to removing or transforming data so that is is meaningful when used in statistical analysis. For example, blood measurements such as cholesterol can be expressed in millimoles per litre $(\mathbf{m m o l} / \mathbf{L})$ or milligrams per decilitre $\mathbf{m g} / \mathbf{d L}$. 
In the UK it is usually the case that the former is used to record cholesterol, however this is not always the case and the units are not attached. Limits of normality must be defined to determine if a reading is likely to expressed in certain units or not by looking at the value recorded. While there is an obvious difference in the values recorded within different units it is possible for two extreme measurements of each of the scale of different units to have a similar value. Some values may even appear outside of any limits of normality of any units of measurement and might require from the study.

Cleansing on measurement values are relatively more straightforward than making assumptions on how other items of data are recorded. Two examples of this are the dates GP records are documented and the reason for admission to secondary care. In the first example you assume that a recording of a prescription in a GP database was made on that date appearing in the records. However this could have been entered by administrative staff retrospectively, or even entered by both the GP and staff where it appears that the same prescription was made on two separate days. Many diagnosis codes for epilepsy appear before electronic records were even integrated into healthcare systems, suggesting that historical information is entered by GPs where the date appearing in the records is an approximation of when someone was first diagnosed with epilepsy.

In the second example, ICD-10 codes are used to record episodes of care in secondary care. For each episode 14 ICD-10 codes may be entered to record the details of care required. This can lead to some interesting codes being used at point of admission as it may be in the interest of hospital staff to include background information about patients to tailor their care through an inpatient stay. Chronic conditions such as diabetes or asthma often get recorded even if they are unrelated to the admission. A distinction has to be made because codes that are entered to detail care required does not directly translate to reason for admission. These 14 diagnostic positions are ordered by priority of care, however this leads to many of the high priority positions being taken up by generic symptoms such as "chest pain" or "out of breath", and for complicated episodes of care there could be multiple valid reasons for admission. There is clearly no "one-size fits all" approach into which positions are used as a proxy for reason for admission as the actual reason for admission is not known. This rules out an opportunity to conduct a sensitivity analysis to determine how many positions are included.

Finally, some other obvious errors appear in large datasets that are common and well known, but nevertheless have to be taken into account. These are errors such as men appearing to be pregnant (incorrect gender code assigned), patients seeing their 
GP after they have died (incorrect date of death) and people still alive longer than the known human life span (incorrect date of birth). A particularly painful error to identify in large datasets are when NHS numbers are incorrectly entered at point of care. For example there are records in SAIL that suggest one person has seen two different GPs in different health boards on the same day, where this behaviour can be traced back for numerous years. While it isn't impossible for this to be happen, it is most likely that the multiple persons are sharing an NHS number due to a mistake at the point of registration. In this instance cross checking with other datasets may help determine which person the NHS number truly belongs to, and therefore which set of records to exclude.

All of these cleansing considerations were taken into account for the various studies in this thesis when using the SAIL databank.

\subsubsection{Statistical Analysis}

The SAIL gateway contains various software packages to analyse linked datasets in

SAIL. Studies in this thesis use the open source R statistical software language to produce statistics and figures. SAIL has strict guidelines on what data is allowed to be taken out of the secure gateway and be included in publications. The results of statistical analysis must not contain data that could potentially identify a person, even from anonymous data. Therefore number of persons in group outputs may not be reported below 5 persons as persons in this group could potentially be identified through linking many datasets together and building a detailed patient profile. Individual level data is therefore restricted from being reported as part of any statistical analysis. 


\subsection{Natural Language Processing}

To construct NLP algorithms and validate their ability to extract important items of text from clinic letters, clinic letters were sourced from Morriston Hospital ${ }^{1}$. Patients with known epilepsy were sourced form clinic letters held in the Swansea Epilepsy Database that stores patient data from the Epilepsy unit in Morriston hospital, and patients without epilepsy were sourced from general neurology clinics. All letters were de-identified by replacing identifiable information with fake information. These letters were then made available to aid constructing an automated NLP algorithm to extract epilepsy specific information from the clinic letters. The clinic letter in Figure 2.4 is representative of the clinic letters used in this study.

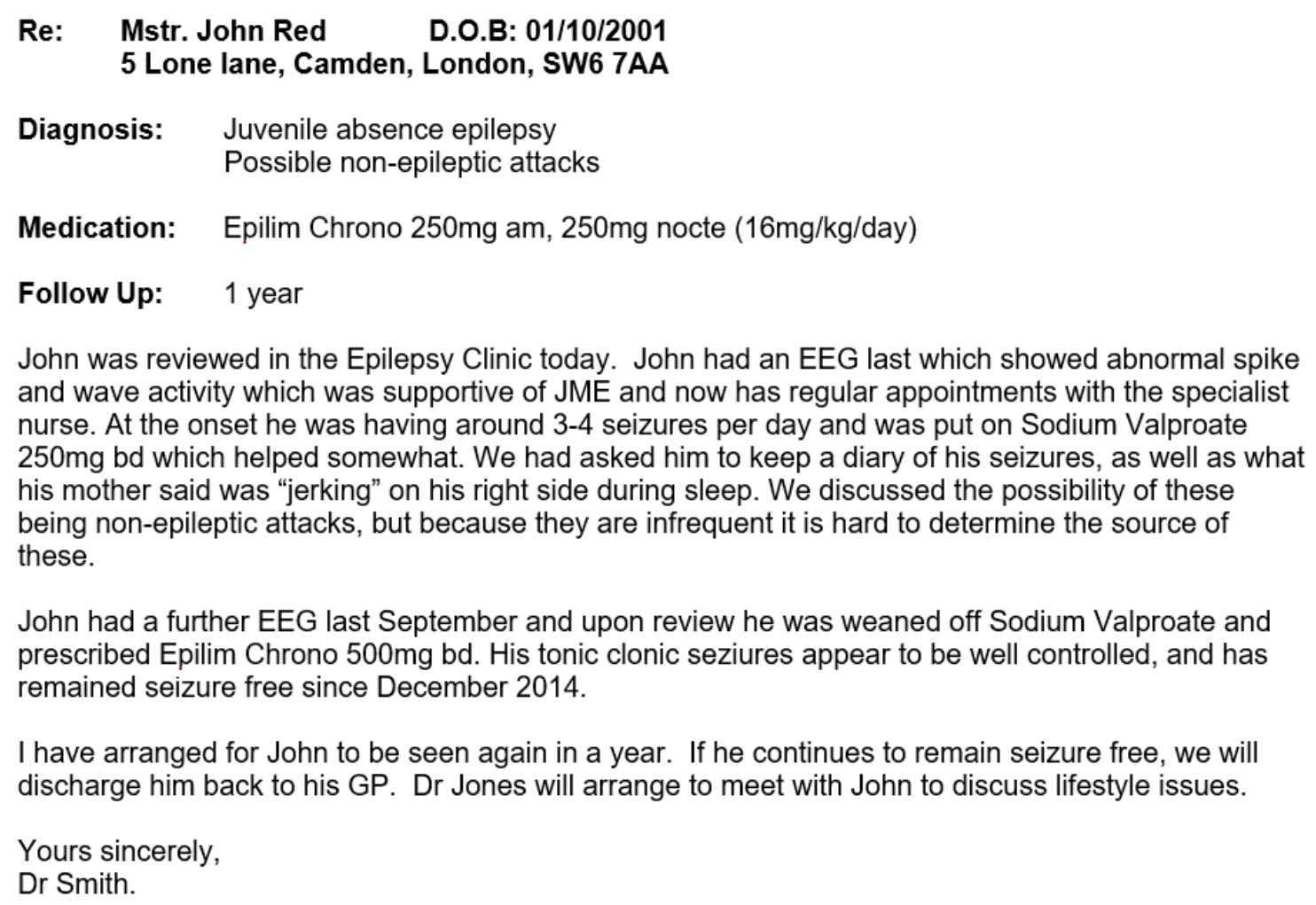
and wave activity which was supportive of JME and now has regular appointments with the specialist nurse. At the onset he was having around 3-4 seizures per day and was put on Sodium Valproate $250 \mathrm{mg}$ bd which helped somewhat. We had asked him to keep a diary of his seizures, as well as what his mother said was "jerking" on his right side during sleep. We discussed the possibility of these being non-epileptic attacks, but because they are infrequent it is hard to determine the source of these.

John had a further EEG last September and upon review he was weaned off Sodium Valproate and prescribed Epilim Chrono 500mg bd. His tonic clonic seziures appear to be well controlled, and has remained seizure free since December 2014.

I have arranged for John to be seen again in a year. If he continues to remain seizure free, we will discharge him back to his GP. Dr Jones will arrange to meet with John to discuss lifestyle issues.

Yours sincerely,

Dr Smith.

Figure 2.4: A example clinic letter. The letter contains real patient data, but all identifiable information has been anonymized

\subsubsection{Software}

The open source GATE v8.4.1 (General Architecture for Text Engineering) https: //gate.ac.uk/ framework was used to construct an algorithm to extract epilepsy specific information from clinic letters, as other details such as clinic date, patient NHS number and date of birth. GATE allows users to build rule sets by combining custom gazetteers (user defined dictionaries) with mechanisms form specifying word

\footnotetext{
${ }^{1}$ performed by Ms Beata Fonferko-Shadrach
} 
ordering by writing JAPE (Java Annotation Pattern Engine) scripts. GATE also provides plugins to perform common NLP tasks such as POS (Part-of-Speech) tagging, NER (Named Entity Recognition) and phrase identification, while also allowing the user to put together such plugins as modules in a pipeline. A GATE pipelines are constructed with a provided GUI (Graphical User Interface).

\subsubsection{Part of Speech tagging}

POS tagging formed the basis of information extraction for this study. Words in text are classified into grammatical units such as verbs, adjectives and nouns. The following phrase:

Mary has focal epilepsy and has been taking Lamotrigine for five years can be tagged in the following way:

$$
\begin{aligned}
& \text { Mary-NNP has-VBZ focal-JJ epilepsy-NN and-CC has-VBZ } \\
& \text { been-VBN taking-VBG Lamotrigine-NNP for-IN five-CD } \\
& \text { years-NNS }
\end{aligned}
$$

where tags are in red. The ANNIE POS Tagger is used to POS tag clinic letters used in the epilepsy NLP algorithm, in which the possible tags are given in table 2.1. 
Table 2.1: ANNIE POS tags and their descriptions

\begin{tabular}{|c|c|}
\hline POS tag & Description \\
\hline $\mathrm{CC}$ & $\begin{array}{l}\text { coordinating conjunction: 'and', 'but', 'nor', 'or', 'yet', plus, minus, less, times } \\
\text { (multiplication), over (division). Also 'for' (because) and 'so' (i.e., 'so that'). }\end{array}$ \\
\hline $\mathrm{CD}$ & cardinal number \\
\hline DT & determiner: Articles including 'a', 'an', 'every', 'no', 'the', 'another', 'any', 'some', 'those'. \\
\hline $\mathrm{EX}$ & $\begin{array}{l}\text { existential 'there': Unstressed 'there' that triggers inversion of the inflected verb and the } \\
\text { logical subject; 'There was a party in progress'. }\end{array}$ \\
\hline FW & foreign word \\
\hline IN & preposition or subordinating conjunction \\
\hline JJ & adjective: Hyphenated compounds that are used as modifiers; happy-go-lucky \\
\hline JJR & $\begin{array}{l}\text { adjective comparative: Adjectives with the comparative ending 'er' and a comparative } \\
\text { meaning. Sometimes 'more' and 'less'. }\end{array}$ \\
\hline JJS & $\begin{array}{l}\text { adjective superlative: Adjectives with the superlative ending 'est' (and 'worst'). Sometimes } \\
\text { 'most' and 'least'. }\end{array}$ \\
\hline LS & list item marker: Numbers and letters used as identifiers of items in a list. \\
\hline MD & $\begin{array}{l}\text { modal: All verbs that don't take an 's' ending in the third person singular present: 'can', } \\
\text { 'could', 'dare', 'may', 'might', 'must', 'ought', 'shall', 'should', 'will', 'would'. }\end{array}$ \\
\hline NN & noun singular or mass \\
\hline NNP & proper noun singular: All words in names usually are capitalized but titles might not be. \\
\hline NNPS & proper noun plural: All words in names usually are capitalized but titles might not be. \\
\hline NNS & noun plural \\
\hline NP & proper noun singular \\
\hline NPS & proper noun plural \\
\hline PDT & $\begin{array}{l}\text { predeterminer: Determiner like elements preceding an article or possessive pronoun such as } \\
\text { 'all/PDT his marbles', 'quite/PDT a mess'. }\end{array}$ \\
\hline POS & possessive ending: Nouns ending in "s' or "'. \\
\hline $\mathrm{PP}$ & personal pronoun \\
\hline PRP & possessive pronoun,such as 'my', 'your', 'his', 'his', 'its', 'one's', 'our', and 'their'. \\
\hline $\mathrm{RB}$ & $\begin{array}{l}\text { adverb: most words ending in 'ly'. Also 'quite', 'too', 'very', 'enough', 'indeed', 'not', 'n't', } \\
\text { and 'never'. }\end{array}$ \\
\hline RBR & adverb comparative: adverbs ending with 'er' with a comparative meaning. \\
\hline RBS & adverb superlative \\
\hline $\mathrm{RP}$ & particle: Mostly monosyllabic words that also double as directional adverbs. \\
\hline STAART & start state marker (used internally) \\
\hline SYM & symbol: technical symbols or expressions that aren't English words. \\
\hline TO & literal "to" \\
\hline $\mathrm{UH}$ & interjection: Such as 'my', 'oh', 'please', 'uh', 'well', 'yes'. \\
\hline VBD & verb past tense: includes conditional form of the verb 'to be'; 'If I were/VBD rich...'. \\
\hline VBG & verb gerund or present participle \\
\hline VBN & verb past participle \\
\hline VBP & verb 3rd person singular present \\
\hline VB & verb base form: subsumes imperatives, initiatives and subjunctives. \\
\hline VBZ & verb 3rd person singular present \\
\hline WDT & 'wh' determiner \\
\hline WPH & possessive 'wh' pronoun: includes 'whose' \\
\hline WP & 'wh' pronoun: includes 'what', 'who', and 'whom'. \\
\hline WRB & 'wh' adverb: includes 'how', 'where', 'why'. Includes 'when' when used in a temporal sense. \\
\hline
\end{tabular}

\subsubsection{Gazetteers}

The GATE framework makes extensive use of dictionaries, or gazetteers to tag words with higher level information than simple grammatical units. The Bio-YODIE plugin for GATE was used to find biomedical references in the clinic letters. Bio-YODIE 
Table 2.2: UMLS representation of a subset of epilepsy terms. The CUI (Concept Unique Identifier) is a code assigned to biomedical concepts. The source column represents the original coding system the term exists in, and the SCUI column is the source code used within a particular system. For example "Epilepsy" exists in both ICD10 and READ coding systems, but map to the same CUI in UMLS despite having unrelated SCUIs.

\begin{tabular}{llll}
\hline CUI & Term & SCUI & Source \\
\hline C0014544 & Epilepsy & G40 & ICD10 \\
C0014544 & Epilepsy & F25 & READV2 \\
C0014549 & Tonic-Clonic Epilepsy & F2510 & READV2 \\
C0014558 & Other Epilepsy & G40.8 & ICD10 \\
C0477371 & Progressive myoclonic epilepsy & F1321 & READV2 \\
C1827284 & Intractable occipital lobe epilepsy & 425054007 & SNOMEDCT_US \\
C1827878 & Refractory localization-related epilepsy & 422724001 & SNOMEDCT_US \\
C1827284 & Refractory occipital lobe epilepsy & 425054007 & SNOMEDCT_US \\
C1827691 & Intractable frontal lobe epilepsy & 425237009 & SNOMEDCT_US \\
C1827974 & Refractory parietal lobe epilepsy & 425349008 & SNOMEDCT_US \\
C1827974 & Intractable parietal lobe epilepsy & 425349008 & SNOMEDCT_US \\
\hline
\end{tabular}

which the UMLS (Unified Medical Language System) - 600 healthcare coding systems such as ICD-10, READ and SNOMED CT combined into a unified coding system to form the basis of a gazetteer that maps any text found in a document to a UMLS code.

The Bio-YODIE plugin scans all text in a document, and where it finds a match to a term the text is tagged and assigned a CUI code (mapping shown in Table 2.2). Custom gazetteers were also defined to incorporate information such as certainty levels ("likely"," probably"," doubtful"....) or hypothetical modifiers ("to see","we may","to determine"...) to help define the context to which UMLS mappings are found.

\subsubsection{Context Algorithm}

The Context algorithm developed by Harkema et al [232] was used to determine if terms are negated e.g. "Mary does not have epilepsy" or if they are attributed to someone other than the patient, such as a family member. The Context algorithm also tags symptoms in terms of their temporal context such as historical or hypothetical. The GATE plugin implements the Context algorithm through multiple gazetteers that contain trigger words for various contexts, such as pre and post negation terms, temporal triggers and triggers for family members. These triggers are related to biomedical terms found in the text by writing rules in the JAPE scripting language. 


\subsubsection{JAPE rules}

Implementing POS tagging and gazetteer mapping provides information ranging from simple grammatical units to meaningful biomedical concepts and contextual terms. The JAPE scripting language was used to weave together sequences of tagged terms to form rules. After tagging, a phrase containing prescription information might look like:

$\begin{array}{lllllllll}\text { He } & \text { is } & \text { taking } & \text { Lamotrigine } & \mathbf{2 5 0} & \text { mg } & \text { twice } & \text { a } & \text { day } \\ \text { PRP } & \text { VBZ } & \text { VBG } & \text { NNP } & \text { CD } & \text { NN } & \text { RB } & \text { DT } & \text { NN } \\ \text { person } & \text { current } & & \text { DRUG } & \text { number } & \text { unit } & \text { word-num } & & \text { temporal } \\ \text { patient } & & & \text { C0064636 } & \text { quantity } & & \text { quantity } & & \text { calendar }\end{array}$

where each word has been assigned multiple tags by mapping to various user defined gazetteers. Further context can be built by combining words, for example "250" and "mg" is a unit of measurement that could be annotated and used for downstream processes.

\begin{tabular}{|c|c|c|c|c|c|c|c|c|}
\hline He & is & taking & Lamotrigine & 250 & mg & twice & $\mathbf{a}$ & day \\
\hline \multirow{4}{*}{$\begin{array}{l}\text { PRP } \\
\text { person } \\
\text { patient }\end{array}$} & VBZ & VBG & NNP & $\mathrm{CD}$ & NN & $\mathrm{RB}$ & DT & NN \\
\hline & current & & DRUG & number & unit & word-num & & temporal \\
\hline & & & C0064636 & quantity & & quantity & & calendar \\
\hline & & & & lvieast & ient & & 90 & cy \\
\hline
\end{tabular}

A JAPE script that could create this rule is shown would be:

In general, larger rules are built by layering smaller rules. For example, if Measurement and Frequency have previously been defined by JAPE rules, those annotations can be used in further JAPE rules.

\begin{tabular}{|c|c|c|c|c|c|c|c|c|}
\hline $\mathrm{He}$ & is & taking & Lamotrigine & 250 & mg & twice & $\mathbf{a}$ & day \\
\hline \multirow{4}{*}{$\begin{array}{l}\text { PRP } \\
\text { person } \\
\text { patient }\end{array}$} & VBZ & VBG & NNP & $\mathrm{CD}$ & NN & $\mathrm{RB}$ & DT & NN \\
\hline & current & & DRUG & number & unit & word-num & & temporal \\
\hline & & & C0064636 & quantity & & quantity & & calendar \\
\hline & & & & Measu & ement & \multicolumn{3}{|c|}{ Frequency } \\
\hline
\end{tabular}




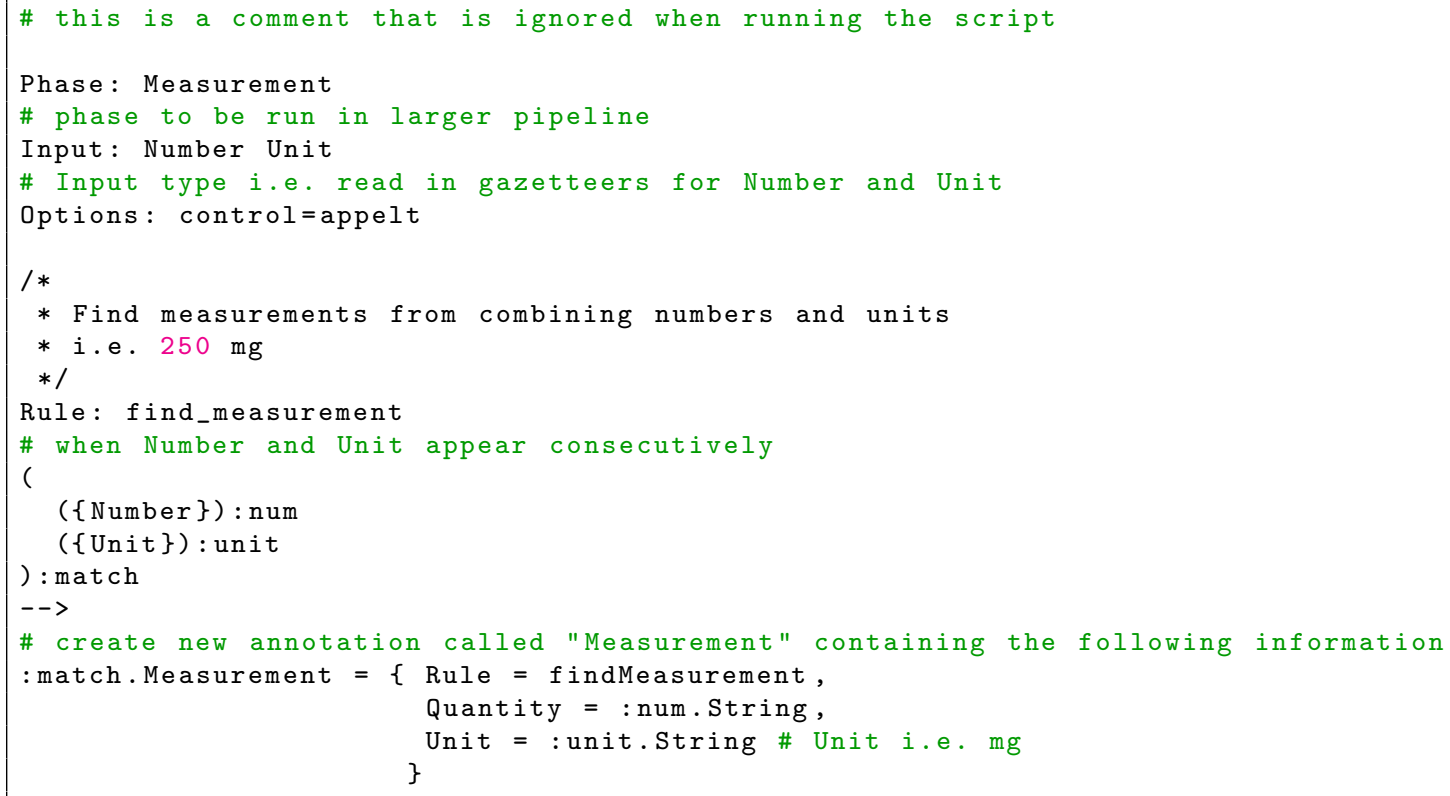

Figure 2.5: A JAPE script to annotate measurements. Gazetteers are used as input to the JAPE script, and depending on the order of words tagged by a gazetteer, a rule can be triggered and create a "Measurement" annotation.

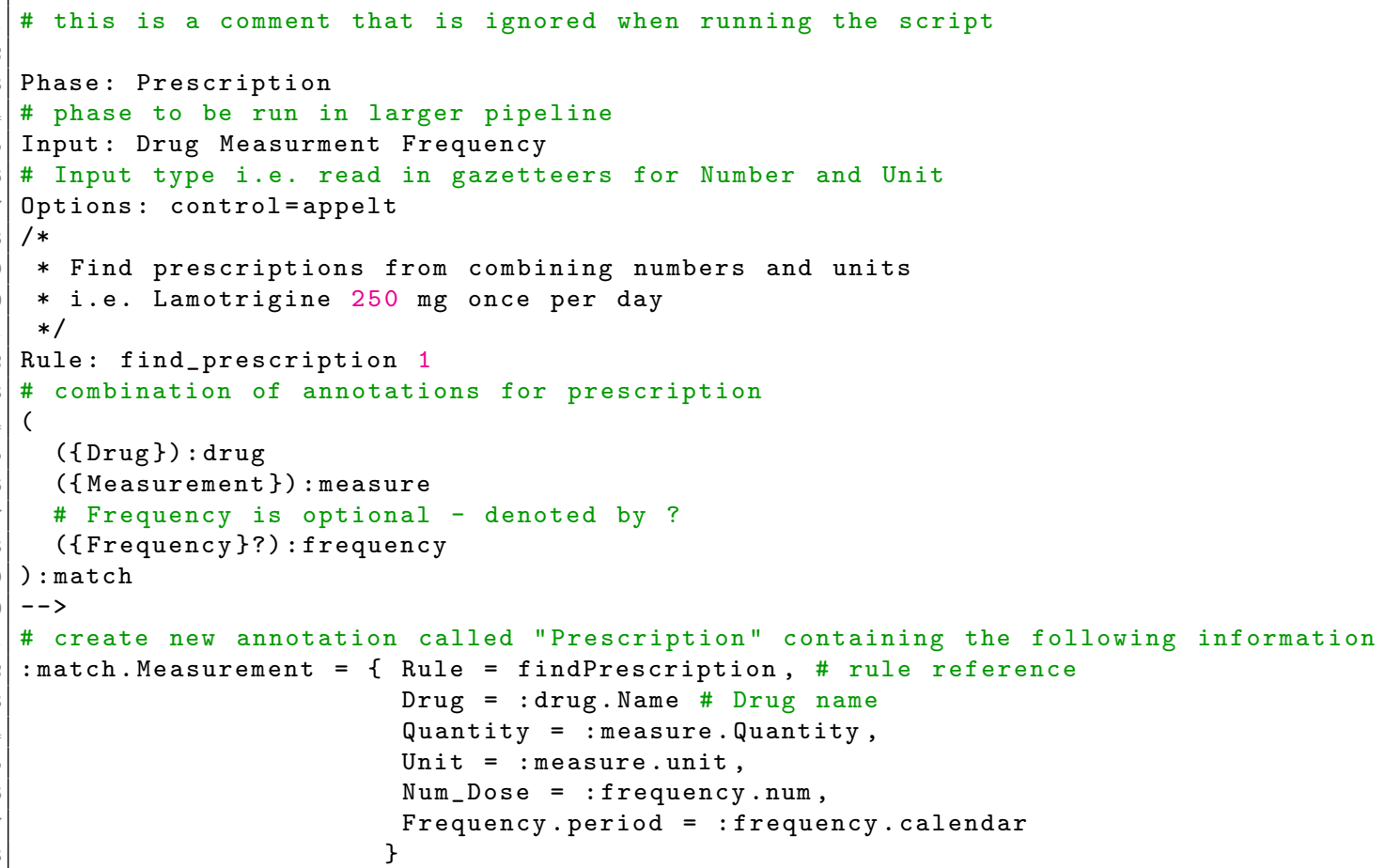

Figure 2.6: A JAPE script to annotate prescriptions. Previous annotations writtin in JAPE rules can be directly used as input to build larger rules. 
The are additional operators than can be used such as the ? operator used in figure 2.6 .

Table 2.3: List of JAPE operators than can be applied to any annotation

\begin{tabular}{ll}
\hline Operator & Description \\
\hline$?$ & optional \\
$*$ & zero or more \\
+ & one of more \\
$!$ & any other than specified annotation \\
{$[\mathrm{x}]$} & exact length of annotation \\
{$[\mathrm{x}, \mathrm{y}]$} & range length of annotation \\
$\mid$ & OR \\
\hline$==$ & AND \\
$!=$ & exact match \\
$==\sim$ & partial match via regex \\
$!=\sim$ & not equal to regex \\
contains & annotation contains specified annotations \\
!contains & annotation doesn't contain other specified anotations \\
within & annotation exists with specified annotation \\
!within & annotation does not exist within specifided annotation \\
\hline
\end{tabular}

\subsection{Predicting functional impact of Single Nucleotide Polymorphisms}

\subsubsection{Pipeline to determine the effect of SNPs}

The SNP pipeline aims to determine the effect of SNPs and their impact on presentation of a disease phenotype, in this case if the SNP is implicated in disease or not. This largely revolves around collecting and processing data that builds a final dataset of protein features for any given SNP, often called annotation. Machine learning methods are applied on the final datasets to predict whether a SNP has the potential to be pathogenic and candidates for disease causality.

\subsubsection{Data sources}

The prediction of functional impact of SNPs largely relied on obtaining biological annotations from publicly available reference data. The main type of data required are meta-data for a given SNP, such as the protein affected, the region that the SNP 
is expressed and the conservation score at the position of the SNP. The following list of databases were used to source SNP features:

- University of California Santa Cruz (UCSC) Genome Browser provides protein level reference data from chromosomal SNP co-ordinates. Datasets used were snp144, kgXref and knownGene. - https://genome.ucsc. edu/.

- Uniprot - Stores various reference data on proteins, in particular the FASTA sequence files - http://www.uniprot.org

- Humvar - a set of SNPs used to train the machine learning algorithms in the pipeline - http://www .uniprot.org/docs/humsavar

\subsubsection{Obtaining Protein Features}

Figure 2.7 describes the various steps in the pipeline to collect the necessary data to make a prediction for a SNP. Given a list of SNPs in chromosomal format each SNP goes through the following pipeline architecture. At each point various cleaning and data wrangling are performed and fed into downstream programs, each accumulating protein features that are useful with regards to protein annotation, but also important for predicting the effects of SNP in terms of disease. 


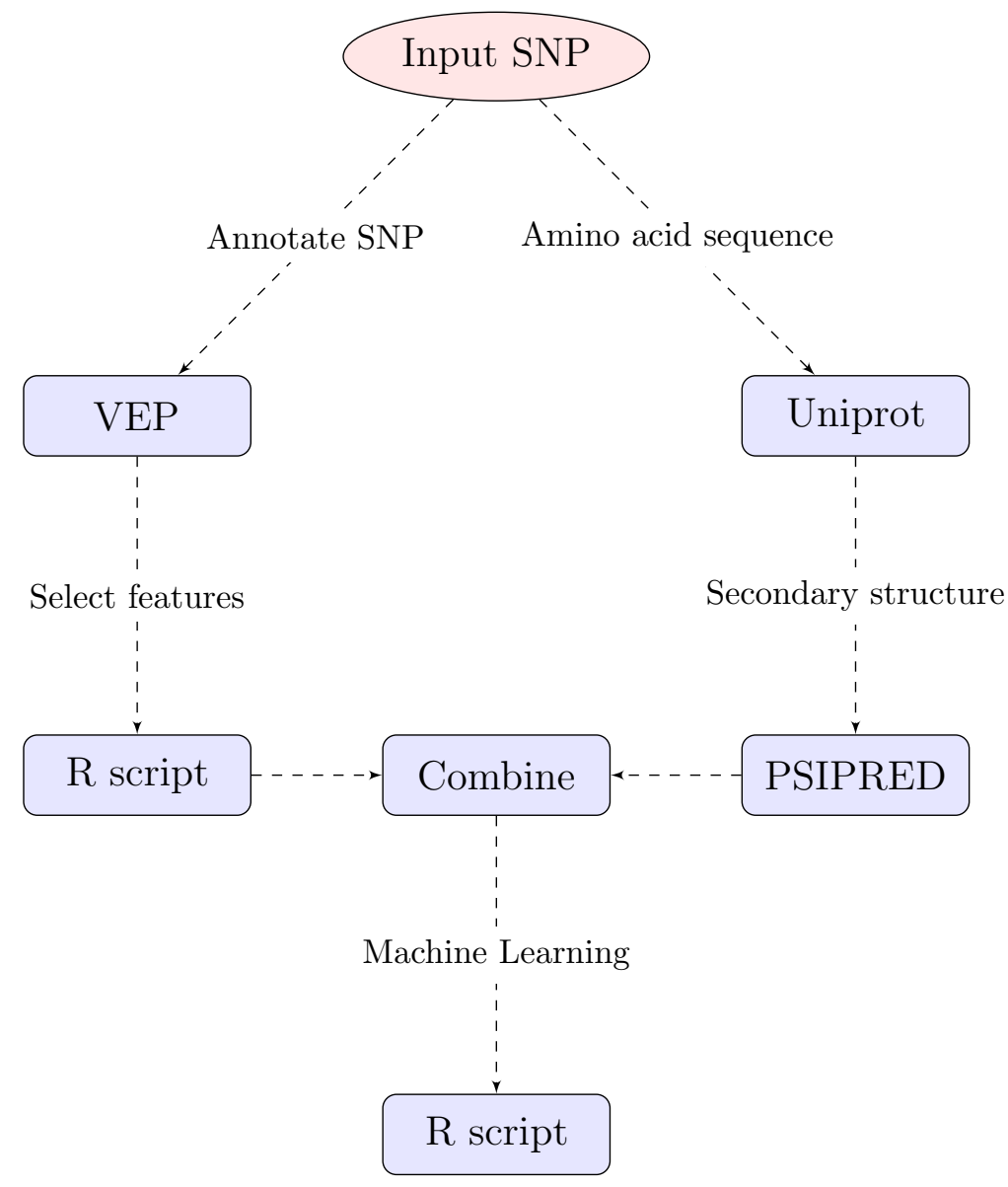

Figure 2.7: A flow chart of the pipeline. Purple nodes are databases and green nodes are processes. The user can specify SNPs in chromosomal format as input to the pipeline. The end result is the SNP data with protein level data that includes indexes generated by downstream programs and database annotation.

\section{SNP format}

SNPs are expressed as chromosomal co-ordinates when called from NGS pipelines. However many protein annotation, prediction and filtering programs require SNPs to be expressed in protein co-ordinates, with various meta-data also included such as gene name and protein ID. The first step of the pipeline is to make this conversion. There are various methods to do this however the conversion was performed on many thousands of SNPs without human interaction. The University of California Santa Cruz (UCSC) genome browser hosts an online database that can be queried with MYSQL scripts. With the chromosome number, position, wild type and mutation nucleotides it was possible to search for the protein co-ordinates and gene information using the code in figure 2.8 .

An important piece of data for this pipeline are the raw amino acid sequences in the form of FASTA files. The protein IDs in the previous script can be used in conjunction 


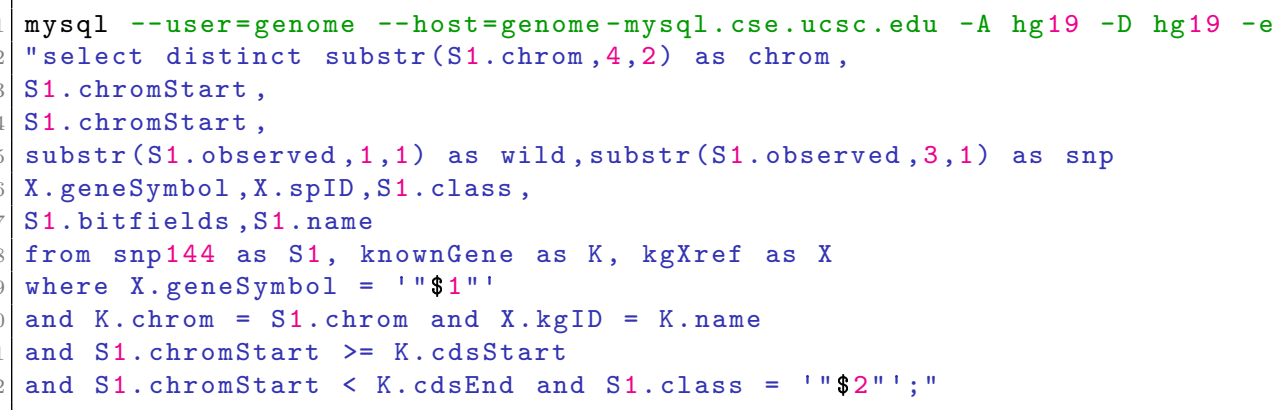

Figure 2.8: MYSQL script to retrieve protein descriptors from chromosomal position.

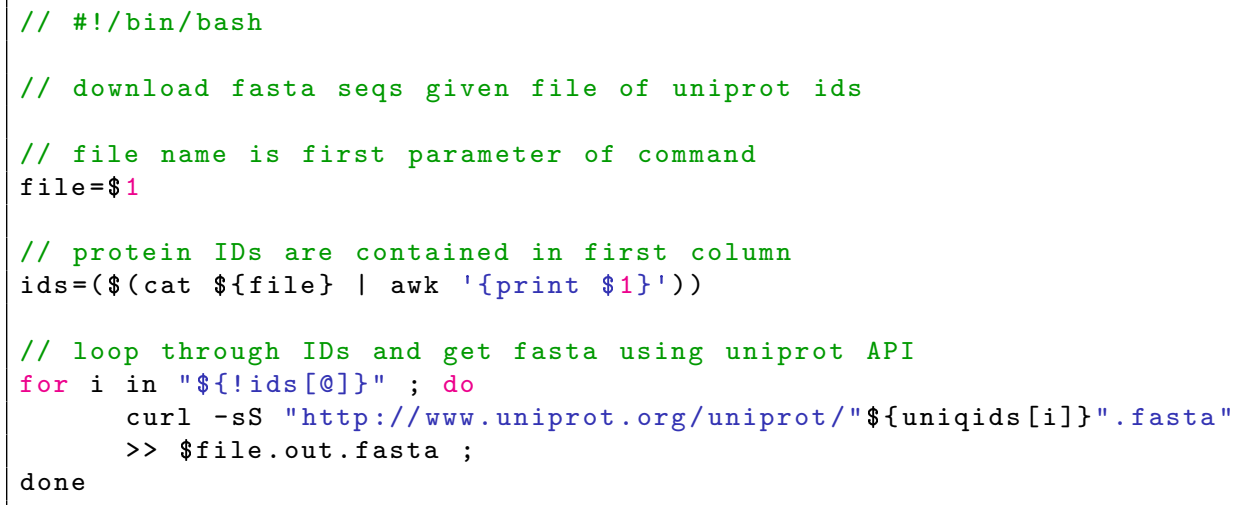

Figure 2.9: FASTA file retrieval using the Uniprot API. SNPs are substituted into each sequence and passed onto downstream programs

with the Uniprot web interface to retrieve FASTA files. These can essentially be accessed via a unique uniprot URL i.e. http://www. uniprot.org/uniprot/P23415. fasta. These can either be typed into an internet browser or programmatically retrieved using webscraping tools such as GNU cURL. Figure 2.9 shows how cURL can be implemented via a bash script to download fasta sequences from the Uniprot API.

This again allows automation and no human interaction - providing that the protein IDs are passed on from previous programs. Using the Uniprot web interface also does not require storage of FASTA files on a local computer, where each FASTA file is downloaded in a few seconds. Later on in the pipeline a GNU AWK program is used to programmatically substitute the mutant SNP in place of the wild type. This is particularly useful for structural modelling where higher dimensional structures are built from raw amino acid sequences. 


\section{Protein annotation with Variant Effect Predictor}

Variant Effect Predictor (VEP) https ://www . ensembl .org/Tools/VEP was used to annotate SNPs. VEP accepts SNPs in chromosomal format, bed format or dbSNP rsID and can scan 35 publicly available datasets ranging from SNPs reported in national GWAS studies, functional prediction scores, binding site locations and regional sub-sequences within proteins. The following features (dataset name) are collected using VEP and used for functional prediction in the pipeline:

- Conserved genomic elements (phastCons,siPhy,GERP)

- Binding sites

- Cytogenic band

- Variants disrupting microRNAs

- Variants disrupting binding sites

- Reported structural variants

- SNP predictions from existing tools

- 1000 genomes frequency annotations

- ExAC frequency annotations

- gnomAD frequency annotations

The last 3 items are used to filter SNPs - frequency based filtering from 1000 Genomes and ExAC allow common SNPs, rare SNPs and unseen SNPs to be separated, where SNPs found in DbSNP contain any published links to clinically observed pathogenicty.

\section{Existing prediction programs}

The scores of existing programs for a given SNP is used for both comparison against the algorithm developed as part of this thesis, but also used as input features to the classification process. Many of these programs provide bespoke protein features used with each algorithm and vary between higher sensitivity and specificity and so are useful to include as input features. The Variant Effect Predictor algorithm was used to annotate SNPs against the dbNSFP https://sites.google.com/site/jp opgen/dbNSFP and table 2.4 lists all of the existing SNP prediction software used as features in the SNP pipeline. 
Table 2.4: List of popular SNP prediction software

\begin{tabular}{ll}
\hline Name & Website \\
\hline Polyphen2 & http://genetics.bwh.harvard.edu/pph2 \\
SIFT & http://sift.jcvi.org/ \\
FATHMM & http://fathmm.biocompute.org.uk/ \\
Provean & http://provean.jcvi.org/index.php \\
MetaSNP & http://snps.biofold.org/meta-snp/ \\
LRT & http://www.genetics.wustl.edu/jflab/index.html \\
MutationTaster & http://www.mutationtaster.org/ \\
MutationAssessor & http://www.mutationtaster.org/ \\
FATHMM & http://fathmm.biocompute.org.uk/ \\
MetaSVM & http://wglab.org/research \\
MetaLR & http://wglab.org/research \\
VEST - & http://karchinlab.org/apps/appVest.html \\
CADD & http://cadd.gs.washington.edu/ \\
GERP++ & http://mendel.stanford.edu/SidowLab/downloads/gerp/ \\
DANN & https://cbcl.ics.uci.edu/public_data/DANN/ \\
fitCons & http://compgen.cshl.edu/fitCons/ \\
Phylop & http://ccg.vital-it.ch/mga/hg19/phylop/phylop.html \\
SiPhy & http://portals.broadinstitute.org/genome_bio/siphy/index.html \\
REVEL & https://sites.google.com/site/revelgenomics/ \\
\hline
\end{tabular}

\section{Predicting secondary structure using PSIPRED}

There are many secondary structure prediction servers that accurately predict which amino acids in a sequence belong to certain types of secondary structural folds, namely beta sheets, alpha coils and helices. The Critical Assessment of protein Structure Prediction (CASP) [233] is a community driven initiative to enhance knowledge of structural prediction. Held bi-annually from 1994, many open source applications have been developed and tested for comparison, where the PSIPRED structural prediction server [225] has regularly featured as one of the most accurate programs of predicting secondary structure from amino acid sequences. PSIPRED is used in the pipeline to predict the difference in secondary structure change between wild type amino acid sequences and sequences with a SNP substituted in. Due to the relatively long processing time (between 15-30 minutes per sequence), the part of the pipeline where PSIPRED is run is hosted on the HPC Wales cluster. This reduces running time to a few minutes per sequence, where sequences can also be run in parallel across the many compute nodes within the HPC Wales cluster.

PSIPRED uses PSIBLAST alignments of proteins with know secondary structure 


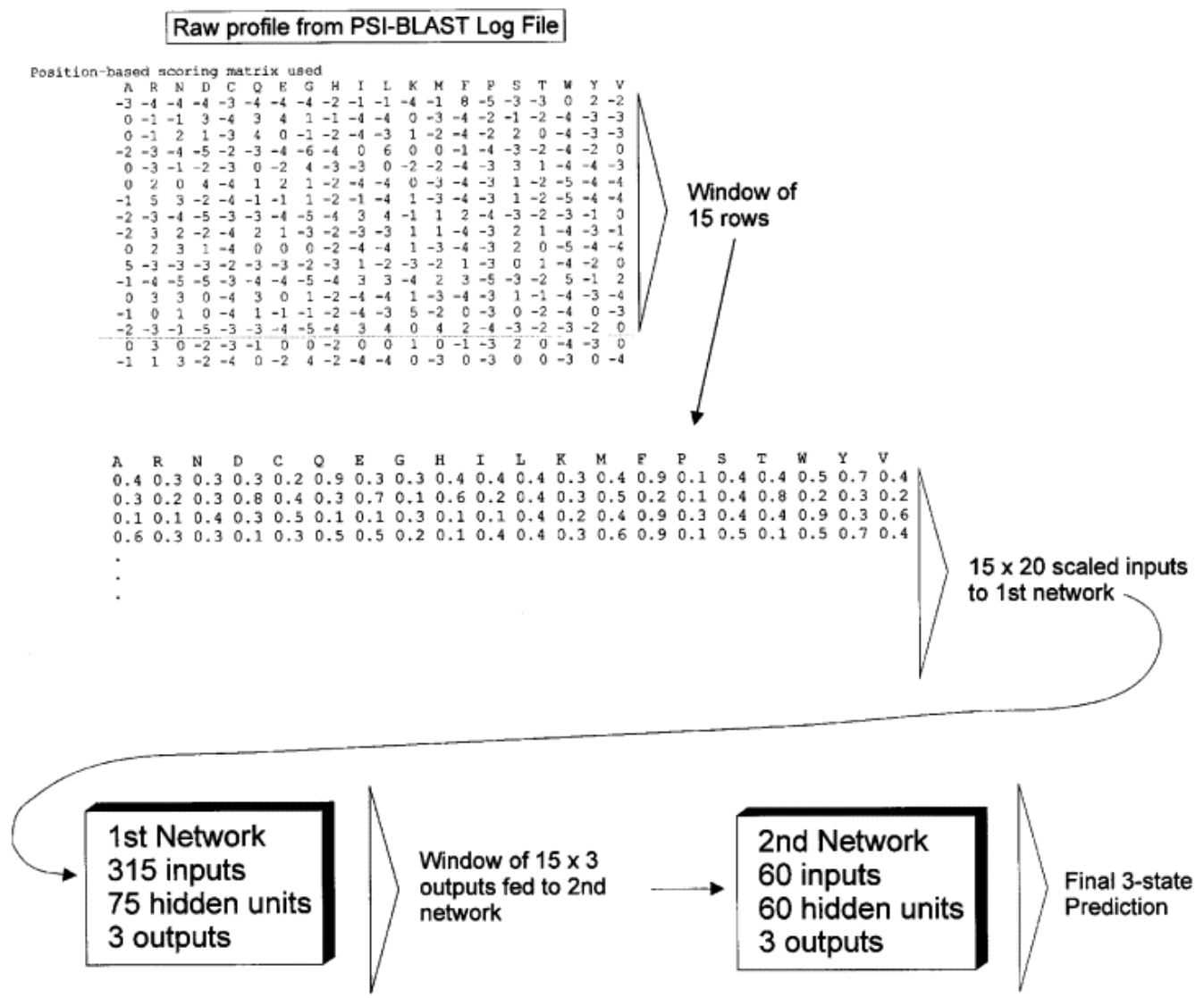

Figure 2.10: PSIPRED algorithm: multiple sequence alignments of known protein structures are built up from an input sequence. Position specific scoring matrix is used to train a neural network to predict the secondary structure of novel proteins.

and then trained with a two-step artificial neural network [234]. For a given protein sequence, PSIBLAST efficiently finds similar sequences via string matching and aligns all the sequences where a scoring matrix is calculated to determine the similarity of each position of each sequence. These position specific scores are used as input to the two stage neural network, along with the ground truth of known secondary structure. The most likely predicted secondary structure fold at each point along the multiple sequence alignment is then assigned by the output of the neural network. It is important to note that the predicted secondary structure at each amino acid in the sequence is determined by which proteins are assigned to the multiple sequence alignment by PSIBLAST. Because PSIBLAST searches on sub-sequences of proteins before building up the alignment, SNPs have the potential to source a small set of proteins not found in an alignment built from the wild type. This small set of proteins can make a difference (albeit small) in the final prediction.

PSIPRED requires amino acid protein sequences in FASTA file format. To compare the output of wild type protein sequences and sequences containing a SNP, the AWK script in figure 2.12 was written to automatically substitute in the SNP to the wild 


$\begin{array}{rllrrr}\text { POS } & \text { AA } & \text { SS } & \text { \%COIL } & \text { \%HELIX } & \text { \%SHEET } \\ 350 & \text { R } & \text { H } & 0.067 & 0.931 & 0.027 \\ 351 & \text { L } & \text { H } & 0.078 & 0.911 & 0.052 \\ 352 & \text { R } & \text { H } & 0.125 & 0.91 & 0.02 \\ 353 & \text { R } & \text { H } & 0.098 & 0.912 & 0.02 \\ 354 & \text { R } & \text { H } & 0.133 & 0.857 & 0.037 \\ 355 & \text { Q } & \text { H } & 0.137 & 0.887 & 0.037 \\ 356 & \text { K } & \text { H } & 0.261 & 0.755 & 0.026 \\ 357 & \text { R } & \text { H } & 0.367 & 0.699 & 0.013 \\ 358 & \text { Q } & \text { C } & 0.51 & 0.49 & 0.028 \\ 359 & \text { N } & \text { C } & 0.698 & 0.275 & 0.028 \\ 360 & \text { K } & \text { C } & 0.725 & 0.245 & 0.044\end{array}$

$\begin{array}{llccr}\text { AA } & \text { SS } & \text { \%COIL } & \text { \%HELIX } & \text { \%SHEET } \\ \text { R } & \text { H } & 0.06 & 0.938 & 0.024 \\ \text { L } & \text { H } & 0.075 & 0.902 & 0.044 \\ \text { R } & \text { H } & 0.111 & 0.933 & 0.018 \\ \text { R } & \text { H } & 0.077 & 0.943 & 0.017 \\ \text { R } & \text { H } & 0.206 & 0.746 & 0.035 \\ \text { R } & \text { H } & 0.171 & 0.812 & 0.047 \\ \text { K } & \text { H } & 0.297 & 0.667 & 0.04 \\ \text { R } & \text { H } & 0.437 & 0.527 & 0.021 \\ \text { Q } & \text { C } & 0.586 & 0.344 & 0.037 \\ \text { N } & \text { C } & 0.721 & 0.251 & 0.025 \\ \text { K } & \text { C } & 0.711 & 0.252 & 0.056\end{array}$

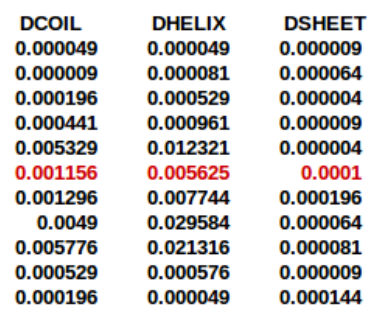

Figure 2.11: PSIPRED output comparing the predicted secondary structure of a wild type GLRA2 sequence with the same sequence having a clinically benign SNP at position 355 of the sequence. The secondary structure prediction is normalised between coil, helix and sheet, where the absolute difference between the wild type and SNP are calculated in the three rightmost columns. The red line indicates the SNP, where other lines are neighbouring amino acids and predictions. It can be seen that while the predicted secondary structure doesn't change, the amino acids closer to the SNP have a larger change in the raw score than those further out from the SNP.

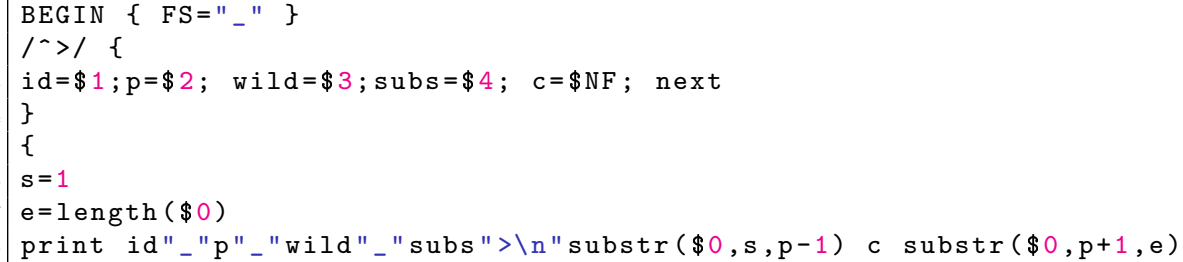

Figure 2.12: An AWK script to substitute a SNP in place of a wild type amino acid within a FASTA sequence. The script takes in 4 parameters that can be read from a text file in the form of 4 columns (protein ID, position of the SNP, amino acid of the wild type, $P$, and the amino acid of the SNP. These are then used tho subsitute the wild type in the position of the SNP with the SNP of the amino acid whilst preserving the original protein flanking either side of the SNP)

type sequence. The fasta header is also modified in this step to contain SNP position, wild type and reference for easy comparison between the wild type and SNP secondary structure output files.

\subsubsection{Predicting SNP Impact Using Machine Learning}

The $\mathrm{R}$ programming language was used to train various machine learning models to classify a SNP as either pathogenic or benign using protein features. A ground truth dataset was established by obtaining protein from the Clinvar ftp://ftp.ncbi .nlm.nih.gov/pub/clinvar/ database for epilepsy SNPs and the Humvar https: //www.uniprot.org/docs/humsavar database for disease non-specific datasets that contain information on a SNP known to cause disease or is benign. These datasets were then annotated using the methods described previously.

The following machine learning algorithms shown in Table 2.5 were used and tested 
on both datasets:

Table 2.5: Machine learning algorithms used in SNP prediction in chapter 5

\begin{tabular}{lll}
\hline Classifier & R package & CRAN link \\
\hline Random Forest & randomForest & https://cran.r-project.org/web/packages/randomForest/randomForest.pdf \\
C45 Decision Tree & rpart & https://cran.r-project.org/web/packages/rpart/rpart.pdf \\
Support Vector Machine & e1071 & https://cran.r-project.org/web/packages/e1071/index.html \\
Logistic Regression & base $\mathrm{N}$ & $\mathrm{NA}$ \\
Artifical Neural Networks & nnet & $\mathrm{https://cran.r-project.org/web/packages/nnet/nnet.pdf}$ \\
Naïve Bayes & naivebayes & https://cran.r-project.org/web/packages/naive-bayes \\
\hline
\end{tabular}

\subsubsection{Training and testing}

The machine learning process was split into 2 phases: training and testing. The training phase used $75 \%$ of the humvar data to train each classifier with the remaining $25 \%$ used as an unseen test set to predict pathogenicty status. A sampling method called cross validation was used where the $75-25 \%$ split is selected randomly, in this case 5 times so that 5 unique models are generated on 5 unique training-test sets.

\subsubsection{Receiver Operator Curves}

Receiver Operator Curves (ROC) are used as a means to validate the accuracy of each classifier. Figure 5.11 shows a ROC curve for multiple classifiers: 


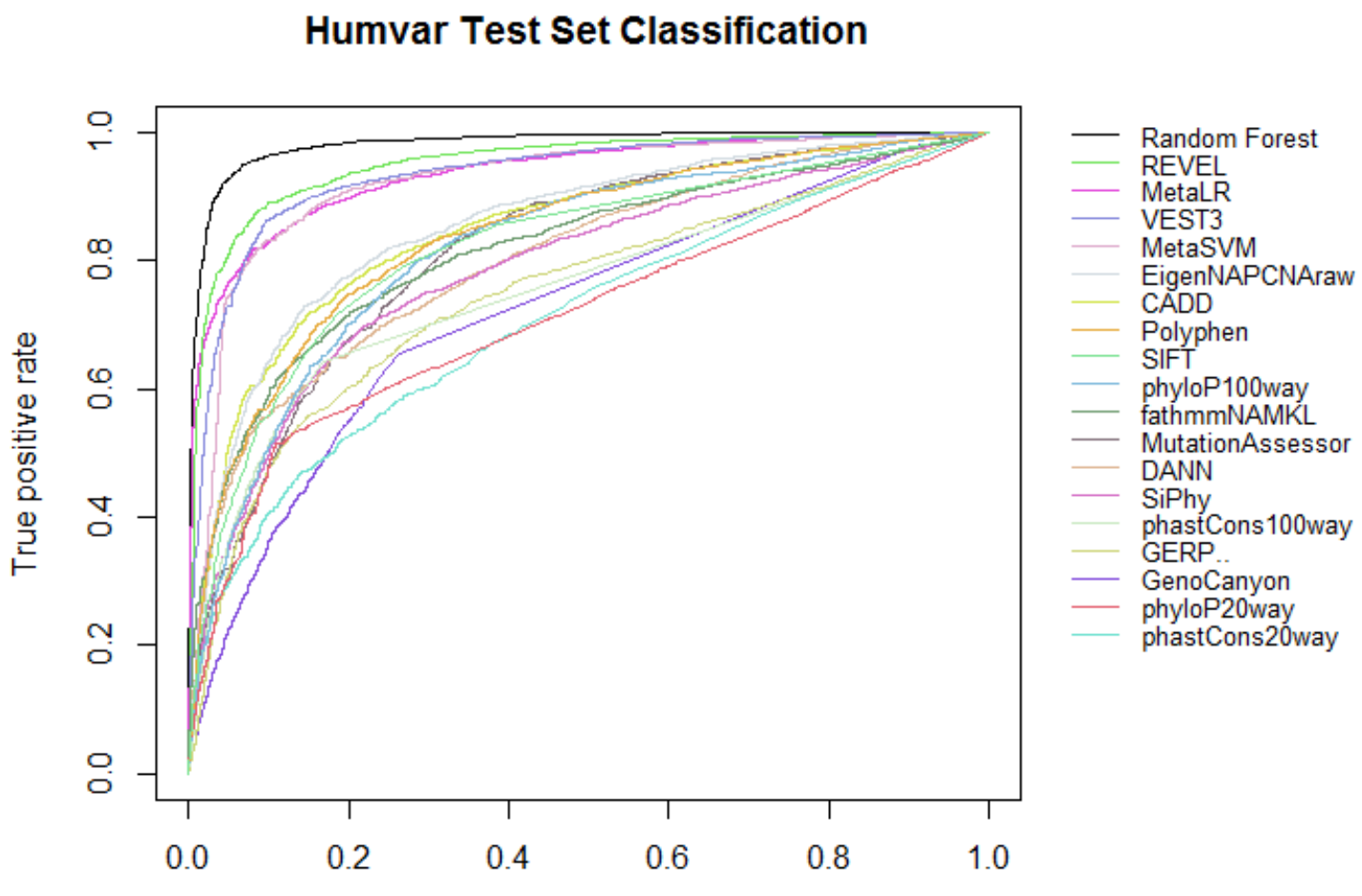

False positive rate

Figure 2.13: ROC curve comparing the classifier from this thesis (black) to scores from other classifiers when predicting disease/benign status on the humvar test set

ROC curves are useful in that each point in the curve plots the sensitivity vs specificity of an algorithm at discrete scoring thresholds. The result is a curve in which the ideal sensitivity vs specificity point can be read off ( i.e. $95 \%$ sensitivity or $95 \%$ specificty) and reproduced for unseen samples with the associated threshold value. Two other interesting properties of ROC curves are the Area Under the Curve measure - a measure of accuracy of the classifier, and the two diagonal lines indicating the line of chance (bottom left to top right) and the line of accuracy (bottom right to top left). Classifiers can easily be compared to the line of chance to see how better it performs than random choice, and the section of the ROC curve that intersects with the line of accuracy is the point denoting the highest accuracy achievable by the algorithm. A ROC curve approaching the top left of the graph indicates a perfect score.

\subsection{Chapter Summary}

- The SAIL Databank was used to carry out retrospective longitudinal studies in people with epilepsy

- SQL queries were used extensively to link datasets together within the SAIL 
databank and extract data in a format ready for statistical analysis

- The R programming was used to carry out statistical analysis

- 240 epilepsy clinic letters from Morriston hospital were used to conduct an NLP study for extracting epilepsy specific information from clinic letters

- The open source GATE application was used as a framework for NLP development. The main concepts behind algorithm development was the inclusion of dictionaries used for tagging terms of interest, such as UMLS codes and writing JAPE scripts that declare rule sets to produce annotations based on tagged terms. Standard NLP applications are also used as part of GATE such as tokenization, POS tagging and chunking

- An epilepsy clinician reviewed 200 test letters and these were compared against the algorithm

- Two SNP datasets were used to test a bespoke SNP classifier for pathogenic/benign status. The Humvar data contains over 70,000 disease non-specific SNPs that were used to trian the classifier, and the Clinvar dataset was used to obtain epilepsy SNPs

- Various open source bioinformatics programs were used for SNP annotation data. The Varaint Effect Predictor was used to annotate SNPs with conservation and frequency data as well as existing SNP prediction scores. PSIPRED was used to calculated the difference in secondary structure prediction between wild type sequences and mutation sequences

- Machine learning was used to test various classifiers, of which this was programmed in $\mathrm{R}$. These were compared against existing scoring software obtained from dbNSFP annotations from VEP 


\section{Chapter 3}

\section{Results: Analysing Routinely Collected Healthcare Records for Epilepsy Research}

The first of the three results chapters show how epilepsy and its impact on health and social factors can be studied using routinely- collected health records in the SAIL databank. Using Welsh GP records an algorithm was created to determine people with robustly-confirmed epilepsy. By comparing GP records in SAIL to patient details in the paediatric neurology department in Morriston Hospital, the algorithm is able to categorise $85 \%$ of patients as having epilepsy while excluding nearly all cases where a lack of clinical evidence confirms the absence of an epilepsy diagnosis . Antiepileptic drugs (AEDs) are studied in terms of how GPs prescribing habits have changed over time, as well adverse effects of AEDs including weight gain and cognitive decline in children born to mothers prescribed AEDs during pregnancy. The relationship of social deprivation and epilepsy is explored where people diagnosed with epilepsy tend to come from areas of higher social deprivation. This chapter presents the strengths and limitations of studying the impact of epilepsy using routinely-collected data. Each study formed the basis of a published paper, in which footnotes are used to account for any specific work undertaken by co-authors.

\subsection{Prevalence, Incidence and the Social Deprivation Profile of Epilepsy in Wales}

The aim of this retrospective study was to determine if prevalence and incidence of epilepsy is due to social drift or social causation by using GP records and demographic 
data between 2004-2010. The first objective was to identify people with epilepsy within SAIL. The GP dataset in SAIL (see 3.1) contains READ codes pertaining an interaction with a GP that was recorded in a patient's GP record. Various codes can be entered such as diagnoses, prescriptions, symptoms, laboratory tests and medical advice. This dataset was used to query codes for both diagnosis codes for epilepsy as well as anti epileptic drug prescriptions (AED). In discussion with clinicians within the Swansea Neurology Research Group, an appropriate method discussed to extract epilepsy cases was to use a combination of repeat AED and epilepsy diagnosis codes. This would pick people with unresolved epilepsy while also excluding people with AED prescriptions exclusively for mental health disorders and pain management. The use of a repeat AED prescription was also included to exclude uses of diagnosis codes used as a way to recorded suspected diagnoses that require follow up.

Table 3.1: SAILWGPV.EVENT_ALF_E is a table in the SAIL Databank that stores GP patient records.

\begin{tabular}{ll}
\hline Field name & Description \\
\hline PRAC_CD_E & Encrypted General Practice code \\
ALF_E & Anonymous linking field representing an encrypted NHS number \\
WOB & Week of Birth - defaults to Monday of week of birth \\
GDNR_CD & Gender code $1=$ male, $2=$ female, $9=$ unknown \\
LOCAL_NUM & Local number identifier - a unique patient number \\
EVENT_CD_VRS & Determines code type such as READ v2, SNOMED etc \\
EVENT_CD & Recorded clinical information during the event \\
EVENT_VAL & The value associated with the recorded event \\
EVENT_DT & Date of the event \\
EPISODE & Denotes if event is due to ongoing care or first recording of diagnosis \\
SEQUENCE & The number of records for a specific event \\
\hline
\end{tabular}

The EVENT_CD column was queried with a list of READ codes that defined AED prescriptions and epilepsy diagnosis, and the WOB column was used to define week of birth for splitting patients into age bands, particularly between adults and children as a child's social deprivation is unlikely to be effected by an epilepsy diagnosis.

\subsubsection{Defining Epilepsy in the SAIL Databank}

The Quality of Outcomes Framework (QOF) https : //www .nice.org.uk/standardsand-indicators/qofindicators aims to encourage GPs to keep patient records as complete as possible. By providing a paid incentive to use certain READ codes to record patient details, finding patients with diseases such as epilepsy should be 
possible by querying GP records. The usage of READ codes used to record details of patients with epilepsy was explored to determine if it is possible to select people with known epilepsy.

\begin{tabular}{|c|c|c|}
\hline \multicolumn{3}{|c|}{ Patients receiving drug treatment for epilepsy in the last six months } \\
\hline Epilepsy & $\mathrm{F} 25 \%$ & $\begin{array}{l}\text { excluding F250I; F25I I; F25I 6; F256.\%; } \\
\text { F258.-F25A.; F25y4 }\end{array}$ \\
\hline Progressive myoclonic epilepsy & \multicolumn{2}{|l|}{$\mathrm{FI} 321$} \\
\hline Traumatic epilepsy & \multicolumn{2}{|l|}{ SC200 } \\
\hline Patient receiving AEDs & \multicolumn{2}{|l|}{$\mathrm{dn} \%$} \\
\hline Patient receiving AEDs & \multicolumn{2}{|l|}{$\mathrm{d} 26 \%$} \\
\hline \multicolumn{3}{|l|}{ Epilepsy Exceptions* } \\
\hline Patient unsuitable & \multicolumn{2}{|l|}{$9 \mathrm{~h} 6 \mathrm{I}$} \\
\hline Informed dissent & \multicolumn{2}{|l|}{$9 \mathrm{~h} 62$} \\
\hline \multicolumn{3}{|c|}{$\begin{array}{l}\text { Patients age } 16 \text { and over on drug treatment for epilepsy who have a record } \\
\text { of seizure frequency in the previous } 15 \text { months ( } 4 \text { points } 90 \% \text { ) }\end{array}$} \\
\hline Fit frequency & \multicolumn{2}{|c|}{6675} \\
\hline Seizure-free $>12$ months & \multicolumn{2}{|l|}{$667 \mathrm{~F}$} \\
\hline No seizures on treatment & \multicolumn{2}{|l|}{$667 \mathrm{P}$} \\
\hline I-12 seizures a year & \multicolumn{2}{|l|}{$667 Q$} \\
\hline $2-4$ seizures a month & \multicolumn{2}{|l|}{$667 R$} \\
\hline I-7 seizures a week & \multicolumn{2}{|l|}{$667 S$} \\
\hline Daily seizures & \multicolumn{2}{|l|}{ 667T } \\
\hline Many seizures a day & \multicolumn{2}{|l|}{$667 \mathrm{~V}$} \\
\hline \multicolumn{3}{|c|}{$\begin{array}{l}\text { Patients age } 16 \text { and over on drug treatment for epilepsy who have been } \\
\text { seizure-free for last } 12 \text { months recorded in last } 15 \text { months }\end{array}$} \\
\hline Seizure-free $>12$ months & \multicolumn{2}{|l|}{$667 \mathrm{~F}$} \\
\hline Epilepsy resolved. & 21260 & $\begin{array}{l}\text { Note:This is a five-byte code that can only } \\
\text { be used in five-byte systems) }\end{array}$ \\
\hline
\end{tabular}

Figure 3.1: Table 1 of 2 defining QOF codes for recording information on epilepsy in patient records. Table taken from https://www.epilepsy.org.uk/sites/epilepsy/files /primary-care-resource/A18-Tool.pdf 


\begin{tabular}{|c|c|}
\hline \multicolumn{2}{|l|}{ Epileptic seizure-free exception } \\
\hline $\begin{array}{l}\text { Patient on maximal tolerated } \\
\text { anticonvulsant therapy }\end{array}$ & 8BL3 \\
\hline Epilepsy medication review & $8 \mathrm{BIF}$ \\
\hline Epilepsy drug side-effects & 6677 \\
\hline Epilepsy treatment changed & 6678 \\
\hline Epilepsy treatment started & 6679 \\
\hline Epilepsy treatment stopped & $667 \mathrm{~A}$ \\
\hline \multicolumn{2}{|l|}{ Pregnancy advice } \\
\hline Contraception advice read code & 6110 \\
\hline Pre-conception advice read code & 67110 \\
\hline Pregnancy advice read code & 67AF \\
\hline \multicolumn{2}{|c|}{ Pregnancy advice - exception codes } \\
\hline $\begin{array}{l}\text { Contraceptive counselling } \\
\text { inappropriate or declined }\end{array}$ & 8IAg.; 8IB2. \\
\hline $\begin{array}{l}\text { Pre-conception advice } \\
\text { inappropriate or declined }\end{array}$ & 8IAh.; 8IB3. \\
\hline $\begin{array}{l}\text { Pregnancy advice inappropriate } \\
\text { or declined }\end{array}$ & 8IAi:; 8IB4. \\
\hline Hysterectomy and equivalent & $\begin{array}{l}\text { 685H; 685I; 685K; 9O8Y; 7E05.\%; 7E040; 7E042; 7E043; } \\
\text { 7E046; 7E049; 7E04B; 7E04G; 7LOA.\%; 26L3 }\end{array}$ \\
\hline Sterilisation & $\begin{array}{l}\text { 7EI0.\%; 7EIII; 7EII3; 7EII5; 7EI5.\%; 7EI60; 7EI62; 7EIC; } \\
\text { 7EIC3; 7EID0; I59A;ZV25x; ZV252 }\end{array}$ \\
\hline
\end{tabular}

Figure 3.2: Table 2 of 2 defining QOF codes for recording information on epilepsy in patient records.Table taken from https://www.epilepsy.org.uk/sites/epilepsy/files/p rimary-care-resource/A18-Tool.pdf

Using the READ codes tables in figures 3.1 and 3.2 an algorithm based on the presence of an epilepsy diagnosis code and a repeat AED prescription was used to determine if a person is known to be living with epilepsy on a given day by querying their GP records. Figure 3.3 depicts a timeline of how a combination of AED prescriptions and epilepsy diagnosis code entered into a GP record and are used to identify epilepsy, and figure 3.4 provides SQL code to implement the process. 


\section{D1. Find AED prescriptions}

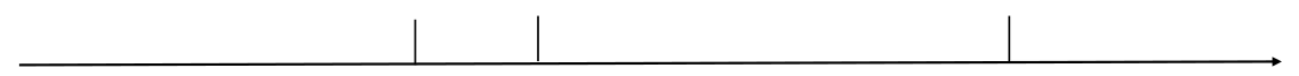

\section{D2. Find repeat AED prescriptions}

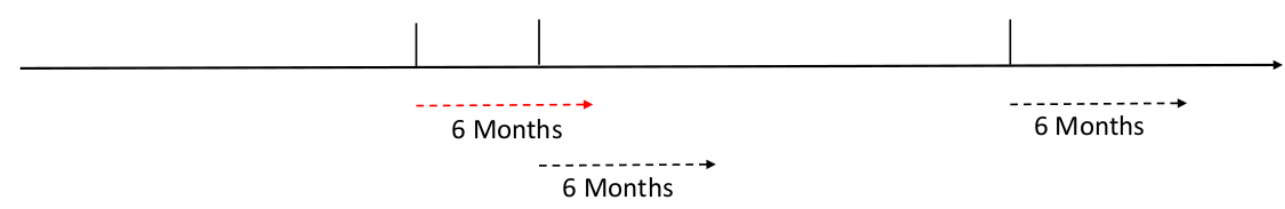

\section{EP. Find epilepsy diagnosis code}

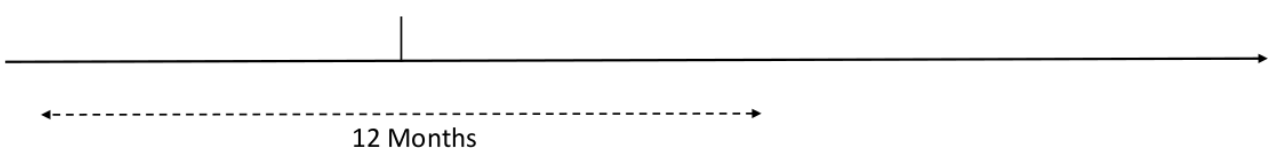

Figure 3.3: Visual explanation of the algorithm used to capture epilepsy diagnoses. All AED prescriptions are first found using GP records (D1), in which AED prescriptions pairs within 6 months after the initial prescription are classed as a repeat AED prescription (D2). Epilepsy diagnosis codes appearing in GP records 12 months either side of the first prescription of each of the AED pairs are queried, and where there is a match a person is classified by the algorithm as having an epilepsy diagnosis at the time of the first AED in the pair.

The SQL query in figure 3.4 was looped through the years 2004-2010, where for those who had a new diagnosis in a given year, all previous years were checked for absence of a diagnosis (incident cases). Those with both new and known epilepsy from previous years that satisfy the extraction criteria in later years contribute to the prevalent population of people with a diagnosis of epilepsy.

The SQL query in 3.4 was also used to determine prevalence and incidence of epilepsy, where prevalence is defined as the number of people with known epilepsy divided by the number of people in the population, and incidence is defined as the number of new cases of epilepsy in a given year divided by the number of people in the population. Annual prevalence between 2004 and 2010 was calculated by identifying the number of people with known epilepsy living in Wales on the 1st of January of a given year and dividing by the total number of people living in Wales on the same day. Annual incidence of epilepsy was also calculated by identifying all newly diagnosed patients in a given year, divided by the total number of persons registered as living in Wales in the same year. 


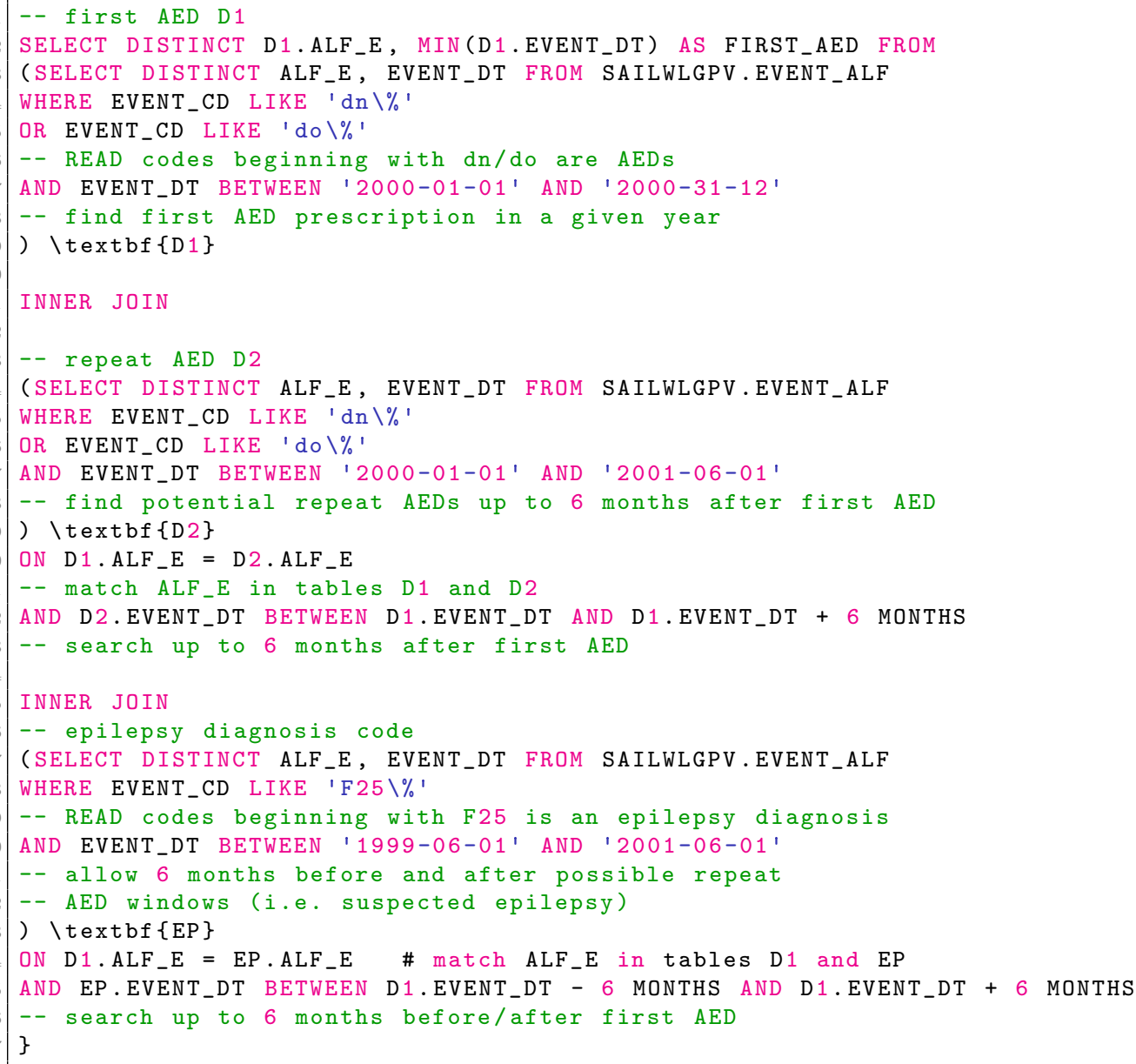

Figure 3.4: An SQL query to extract epilepsy cases using a combination od diagnosis and AED READ codes. In this example, the first known confirmation that a person has epilepsy in 2000 is given by the first AED prescription in their GP record, while also having a repeat AED within 6 months as well as an epilepsy diagnosis up to 6 months either side of the first identified repeat prescription window 


\subsubsection{Social deprivation}

The Welsh Demographic Service dataset was used to obtain social deprivation, which contains WIMD score that are assigned to 1896 geographical Lower Super-Output Areas (LSOAs) in Wales, each with around 1,500 people. Each LSOA is ranked from most deprived to least deprived according to its corresponding WIMD score and then grouped into deciles, with decile 1 being the most deprived and decile 10 the least deprived. For this specific study, WIMD 2011 deciles were used to measure an individuals' social deprivation where for each person in the study it was possible to query their demographic records to determine what LSOA code they live within on any day of the year, in which the 1st of January was chosen for each study year. Tables 3.2 and 3.3 were used to obtain a person's address and link it to their WIMD score by linking the LSOA_CD between both tables:

Table 3.2: The SAILWDSDV.AR_PERS table in the SAIL Databank holds individuals address, provided as the address when registering with a GP. Each address is also assigned to a Lower Super Output Area (LSOA) which is a geographical area comprising of around 1500 individuals.

\begin{tabular}{ll}
\hline Field name & Description \\
\hline PERS_ID_E & Encrypted Person Identifier \\
RALF_E & $\begin{array}{l}\text { Encrypted Residential address sourced from address given } \\
\text { at GP registration }\end{array}$ \\
LSOA_CD & Lower Super Output Area code - approx 1500 person per \\
& area \\
FROM_DT & Day resident started living at address \\
TO_DT & Day resident stopped living at address \\
\hline
\end{tabular}

Table 3.3: The SAILREFRV.WIMD2008_OVERALL_INDEX table in the SAIL Databank contains a link between an LSOA code and various Welsh Index of Multiple Deprivation measures.

\begin{tabular}{ll}
\hline Field name & Description \\
\hline LSOA_CD & Lower Super Output Area code $~ 1500$ people \\
LSOA_DESC & Name of geographic area \\
SCORE & Raw score comprising of 8 indicators \\
RANK & LSOA rank based on raw score. $1=$ most deprived \\
DECILE & Decile LSOA belongs to. $1=$ most deprived \\
QUNTILE & Quintile LSOA belongs to. $1=$ most deprived \\
\hline
\end{tabular}

For each ALF_E in the study it was possible to determine the WIMD Decile at the 


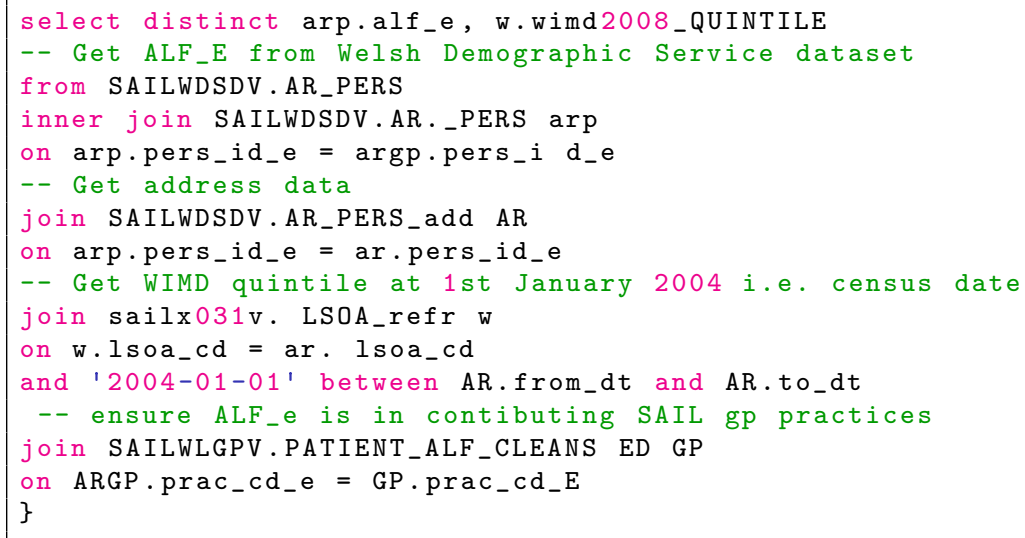

Figure 3.5: An SQL query to link an ALF_E to their address in the Welsh Demographic dataset in SAIL, and how to linke the WIMD quintile to the address on a given day i.e. 1st January 2004

start of each year on the study window using the SQL query in Figure 3.5 and was used to compare the prevalence and incidence epilepsy across WIMD quintiles:

Sex and age were included as covariates where age groups were categorized as $0-5 ; 6-12$; $13-21 ; 22-45 ; 25-45 ; 46-64$, and 65 years or over in relation to their age in the study year. Figure 3.6 shows a summary flowchart of how the cohort was selected and Table 3.4 compares the study population in 2010 with that of the Welsh population.

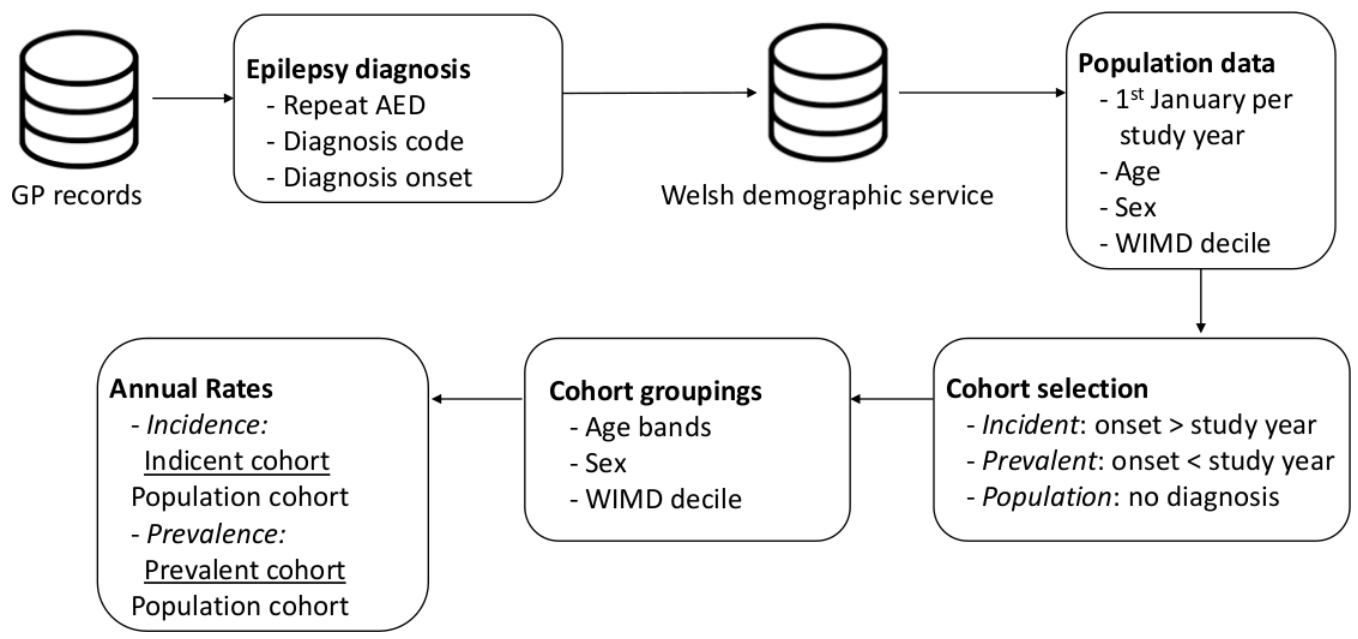

Figure 3.6: Flow chart of cohort selection. GP records were used to identify people with epilepsy (and therefore those that did not have epilepsy). The Welsh Demographic Service dataset was then used to sample age bands, sex and WIMD deciles on the first of January in each study year. For every unique combination of covariates, incidence and prevalence was calculated. 
Table 3.4: Study population characteristics in 2010 as compared to the Welsh population (measured by the 2011 WIMD data). Table taken from [4]

\begin{tabular}{llll}
\hline & & $\begin{array}{l}\text { Study population in } \\
\mathbf{2 0 1 0}\end{array}$ & $\begin{array}{l}\text { Wales } \\
\text { population }\end{array}$ \\
\hline Total Number & & $\mathbf{1 , 1 7 8 , 5 5 8}$ & $\mathbf{3 , 1 6 9 , 5 9 4}$ \\
Sex & Male & $588,476(49.9 \%)$ & $1,582,144(49.9 \%)$ \\
& Female & $590,082(50.1 \%)$ & $1,587,446(50.1 \%)$ \\
Age (years) & $\mathbf{0 - 5}$ & $73,716(6.3 \%)$ & $206,148(6.5 \%)$ \\
& $\mathbf{0 6 - 1 2}$ & $86,809(7.4 \%)$ & $235,681(7.4 \%)$ \\
& $\mathbf{1 3 - 2 1}$ & $142,333(12.1 \%)$ & $367,981(11.6 \%)$ \\
& $\mathbf{2 2 - 4 5}$ & $374,090(31.7 \%)$ & $999,254(31.5 \%)$ \\
& $\mathbf{4 6 - 6 4}$ & $290,612(24.7 \%)$ & $793,247(25.0 \%)$ \\
& $>\mathbf{6 4}$ & $210,998(17.9 \%)$ & $567,282(17.9 \%)$ \\
& $\mathbf{1}$ & $109,703(9.3 \%)$ & $318,275(10.0 \%)$ \\
(Weprivation & & $122,291(10.4 \%)$ & $315,689(10.0 \%)$ \\
& $\mathbf{2}$ & $91,478(7.8 \%)$ & $315,983(10.0 \%)$ \\
& $\mathbf{3}$ & $124,033(10.5 \%)$ & $317,000(10.0 \%)$ \\
& $\mathbf{4}$ & $121,894(10.3 \%)$ & $313,995(9.9 \%)$ \\
& $\mathbf{5}$ & $127,573(10.8 \%)$ & $325,662(10.3 \%)$ \\
& $\mathbf{6}$ & $101,077(8.6 \%)$ & $309,675(9.8 \%)$ \\
& $\mathbf{7}$ & $113,994(9.7 \%)$ & $324,457(10.2 \%)$ \\
& $\mathbf{8}$ & $124,752(10.6 \%)$ & $307,093(9.7 \%)$ \\
& $\mathbf{9}$ & $141,763(12.0 \%)$ & $321,954(10.2 \%)$ \\
\hline $\mathbf{1 0}$ & &
\end{tabular}

Over the study period, the mean epilepsy prevalence was $0.77 \%$ (95\% CI 0.76 to $0.79 \%$ ) and there were 2,390 incident cases of epilepsy, giving a mean incidence rate of 29.5/100,000 per year (95\% CI 28.3 to 30.7). A breakdown of prevalence and incidence for each year is given in Table 3.5. Given that the sensitivity of the epilepsy algorithm in the validation study was $84 \%$ and the prevalence of epilepsy in the UK in 2011 was reported to be $0.97 \%$ by the Joint Epilepsy Council (http://www.epilepsyscotland.org.uk/pdf/Joint_Epilepsy_Council _Prevalence_and_Incidence_September_11_\%283\%29.pdf), the mean prevalence of $0.77 \%$ seems reasonable. 
Table 3.5: Breakdown of epilepsy prevalence and epilepsy incidence by WIMD decile. Table tal

\begin{tabular}{|c|c|c|c|c|c|c|}
\hline \multirow{2}{*}{$\begin{array}{l}\text { Deprivation } \\
\text { (WIMD decile) }\end{array}$} & \multicolumn{3}{|c|}{ Mean epilepsy prevalence 2004-2010 } & \multicolumn{3}{|c|}{ Epilepsy incidence 2004-2010 } \\
\hline & Number of cases & $\begin{array}{l}\text { Mean } \\
\text { population }\end{array}$ & $\begin{array}{l}\text { Mean } \\
\text { prevalence } \\
(\%)\end{array}$ & $\begin{array}{l}\text { Number of } \\
\text { cases }\end{array}$ & $\begin{array}{l}\text { Patient years } \\
\text { at risk }\end{array}$ & Incid \\
\hline 1 & 1211 & 107,464 & 1.13 & 304 & 752,250 & 40.4 \\
\hline 2 & 1164 & 119,990 & 0.97 & 305 & 839,931 & 36.3 \\
\hline 3 & 845 & 89,671 & 0.94 & 228 & 627,696 & 36.32 \\
\hline 4 & 969 & 121,421 & 0.8 & 270 & 849,944 & 31.77 \\
\hline 5 & 989 & 119,345 & 0.83 & 257 & 835,416 & 30.76 \\
\hline 6 & 911 & 125,983 & 0.72 & 247 & 881,878 & 28.0 \\
\hline 7 & 712 & 99,640 & 0.71 & 186 & 697,479 & 26.67 \\
\hline 8 & 742 & 111,456 & 0.67 & 197 & 780,195 & 25.25 \\
\hline 9 & 734 & 122,153 & 0.6 & 208 & 855,069 & 24.33 \\
\hline 10 & 684 & 140,053 & 0.49 & 188 & 980,374 & 19.18 \\
\hline
\end{tabular}


Figure 3.7 shows that the mean prevalence of epilepsy is double in most deprived $(1.13 \%)$ compared to least deprived $(0.49 \%)$, and the mean incidence of epilepsy is also double in most deprived (40.41 per 100,000) compared to least deprived (19.18 per 100,000), identifying a strong trend that epilepsy is associated with increased social deprivation.
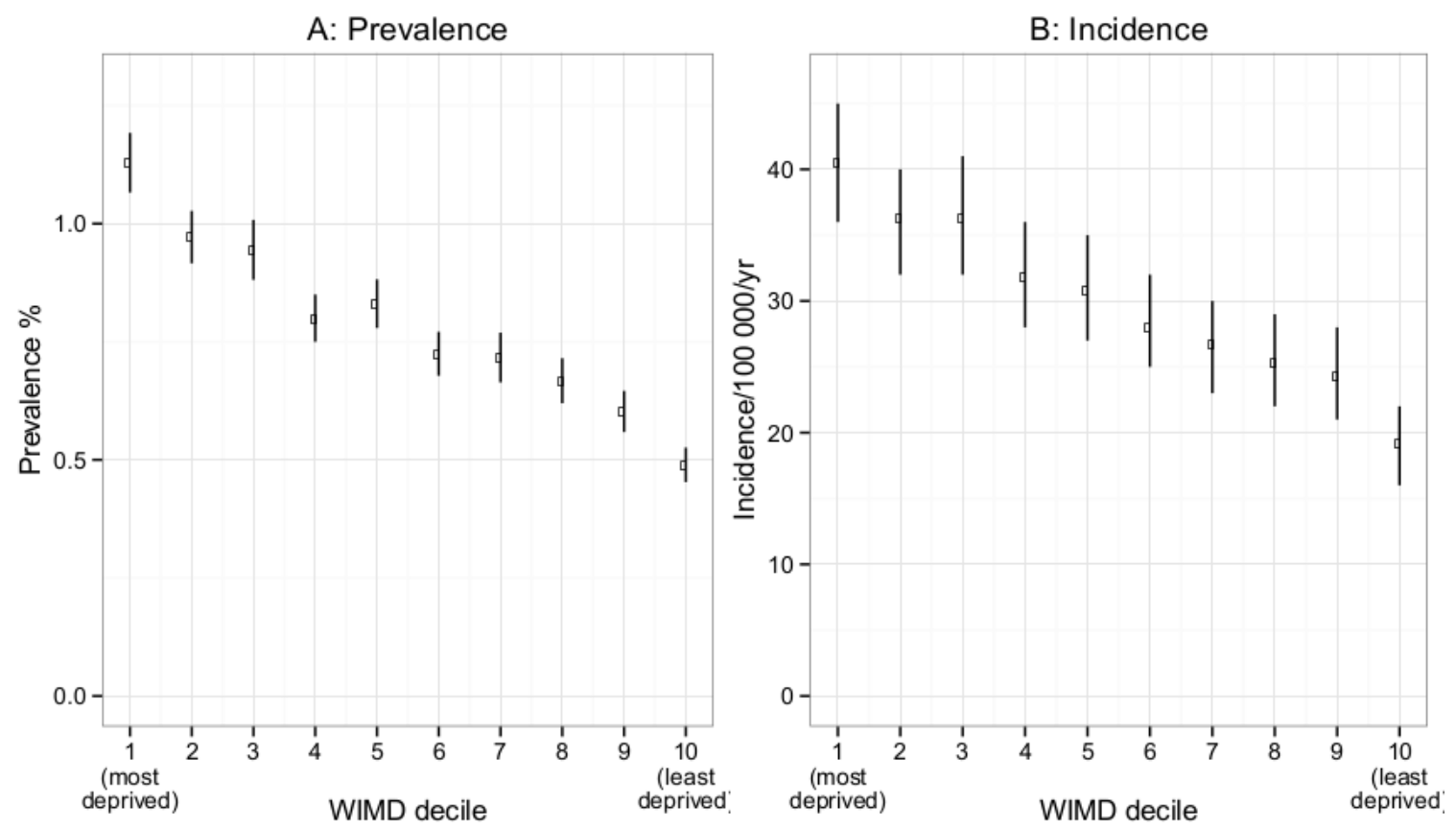

Figure 3.7: Plots of (A) epilepsy prevalence and (B) epilepsy incidence by WIMD (deprivation) decile. Error bars indicate $95 \%$ confidence intervals. Figure taken from $[4]$

The mean prevalence and incidence was calculated for each WIMD decile together with confidence intervals using binomial and Poisson models, respectively. The LSOA WIMD decile data for prevalence and incidence of epilepsy aligned with a 2001 LSOA shape file from the Office of National Statistics https://data.gov.uk/dataset/fa883558-22fb-4a1a-8529cffdee47d500/lower-layer-super-output-area-lsoa-boundaries to produce a geographical representation of deprivation, epilepsy prevalence, and epilepsy incidence in Figure $3.8^{1}$. Geographical areas were excluded where GP information was not available for at least $5 \%$ of the population of that area.

It is possible to see a correlation of deprived areas (dark blue) with areas that have a high prevalence and incidence of epilepsy. Due to densely populated urban areas skewing deprivation on an LSOA level, it can be seen in enlarged portions of the map (Swansea, Cardiff and Newport) that while it is possible to see the correlation

\footnotetext{
${ }^{1}$ With assistance from Dr Joanne Demmler
} 

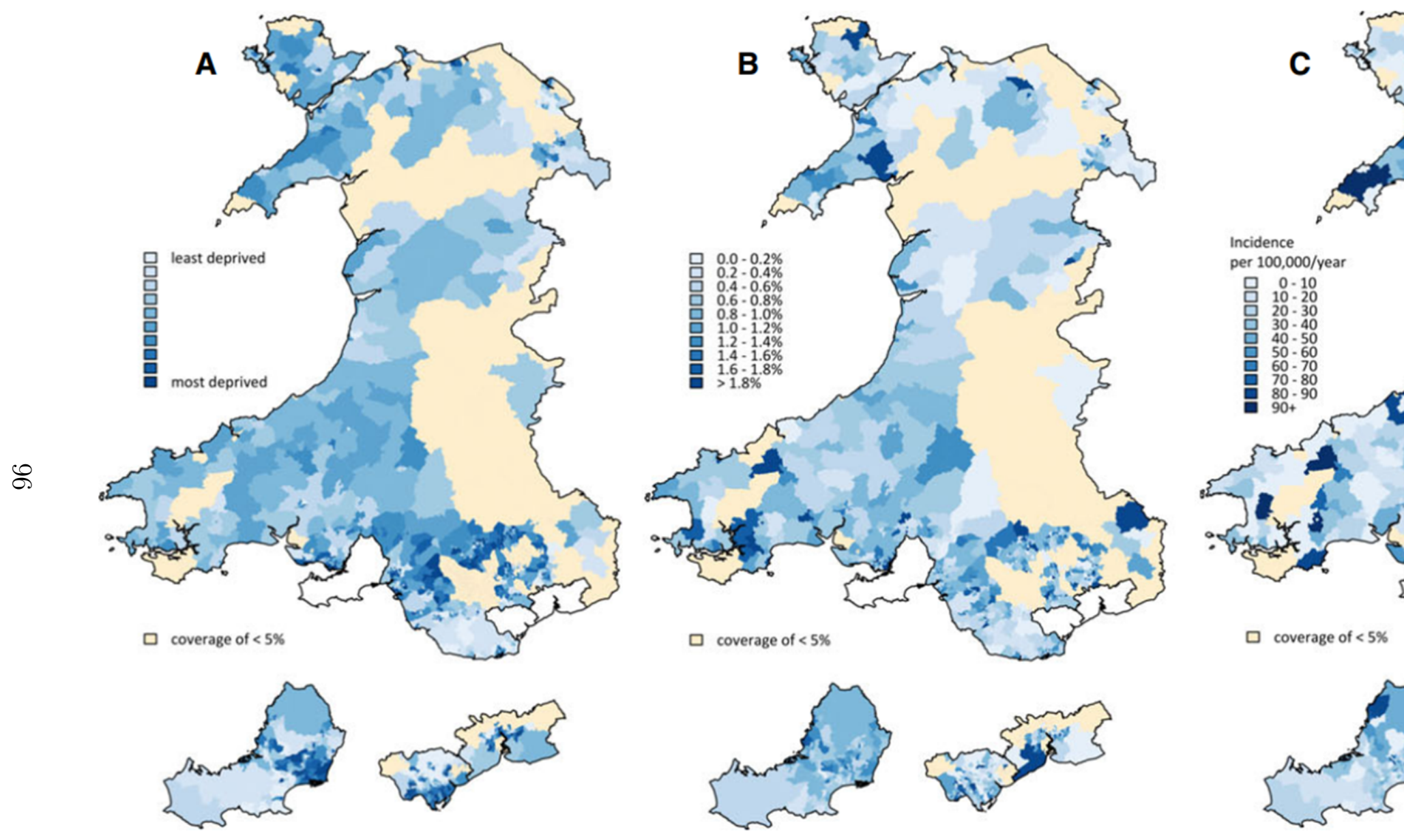

Figure 3.8: Maps of Wales showing each LSOA (areas with population of around 1,500); Yellow areas represent of the population) and are not shown. (A) Deprivation measured by WIMD decile, (B) epilepsy prevalence, Enlarged areas represent the densely populated areas of the cities of Swansea, Cardiff, and Newport (left to rig 
between social deprivation, prevalence and incidence of epilepsy, the correlation is not as clear when viewing Wales as a whole. Odds and incident rate ratios are presented in Table 3.6 for WIMD deciles, sex and age bands, where a WIMD decile of 1, males, and age band 0-5 are used as a reference where ORs and IRs were calculated using multiple logistic regression and Poisson regression models respectively ${ }^{2}$. It can be seen that even after adjusting for WIMD deciles, there is still a significant effect of epilepsy prevalence and incidence.

Table 3.6: Variable Adjusted epilepsy prevalence odds ratio Adjusted epilepsy incidence rate ratio The odds and incidence rate ratios for deprivation (second row of the table) are given per WIMD decile when compared to the population in decile 1 , for example, the odds ratio of epilepsy prevalence in WIMD decile $3=0.922 \times 0.94$ when compared to the population in decile 1. Table taken from [4]

\begin{tabular}{|c|c|c|}
\hline Variable & $\begin{array}{l}\text { Adjusted prevalence odds } \\
\text { ratio }\end{array}$ & $\begin{array}{l}\text { Adjusted incidence rate } \\
\text { ratio }\end{array}$ \\
\hline $\begin{array}{l}\text { Deprivation (per } \\
\text { WIMD decile) }\end{array}$ & $0.922(0.920$ to $0.925 ; \mathrm{p}<0.001)$ & $0.936(0.923$ to $0.950 ; \mathrm{p}<0.001)$ \\
\hline \multicolumn{3}{|l|}{ Sex } \\
\hline Male & $1.0(\mathrm{ref})$ & $1.0(\mathrm{ref})$ \\
\hline Female & $0.981(0.966$ to $0.997 ; \mathrm{p}=0.018)$ & $0.853(0.787$ to $0.924 ; \mathrm{p}<0.001)$ \\
\hline \multicolumn{3}{|l|}{ Age (years) } \\
\hline $0-5$ & $1.0(\mathrm{ref})$ & $1.0(\mathrm{ref})$ \\
\hline $6-12$ & $2.572(2.372$ to $2.792 ; \mathrm{p}<0.001)$ & $0.999(0.828$ to $1.207 ; \mathrm{p}=0.993)$ \\
\hline $13-21$ & $3.419(3.169$ to $3.694 ; \mathrm{p}<0.001)$ & $0.950(0.799$ to $1.134 ; \mathrm{p}=0.565)$ \\
\hline $22-45$ & $5.570(5.183$ to $5.994 ; \mathrm{p}<0.001)$ & $0.573(0.488$ to $0.676 ; \mathrm{p}<0.001)$ \\
\hline $46-64$ & $6.371(5.928$ to $6.859 ; \mathrm{p}<0.001)$ & $0.567(0.479$ to $0.673 ; \mathrm{p}<0.001)$ \\
\hline$>64$ & $6.778(6.304$ to $7.300 ; \mathrm{p}<0.001)$ & $1.098(0.935$ to $1.296 ; \mathrm{p}=0.261)$ \\
\hline
\end{tabular}

\subsubsection{Follow up cohort}

A cohort of adults aged older than 18 years with a new diagnosis of epilepsy between January 1, 2000 and December 31, 2002 was selected as a follow up cohort to measure any difference in WIMD decile 10 years after their diagnosis. Only adults were selected, as a child's deprivation status is determined by their parents' deprivation status and children move with their parents. For each person in this cohort who remained within the study population, a comparison of WIMD decile between time of diagnosis and either 10 years after diagnosis or time of death was used to test the hypothesis that social drift plays a role in increased deprivation for people with epilepsy.

\footnotetext{
${ }^{2}$ Statistical Analysis performed by Dr Owen Pickrell
} 
Table 3.7: Summary of follow up statistics for 10 year follow up cohort. Table taken fro

\begin{tabular}{|c|c|c|c|c|}
\hline & Number & $\begin{array}{l}\text { Mean length of } \\
\text { follow-up in years } \\
(\mathrm{SD})\end{array}$ & $\begin{array}{l}\text { Mean length of } \\
\text { follow-up in years } \\
(\mathrm{SD})\end{array}$ & $\begin{array}{l}\text { (Mean } \\
\text { Decile }\end{array}$ \\
\hline All & 582 & $52.42(20.2)$ & $7.9(3.3)$ & $0.04(\mathrm{r}$ \\
\hline Alive & 352 & $42.96(16.4)$ & $10(0.0)$ & -0.02( \\
\hline Younger than 41 years at diagnosis & 172 & $28.85(6.4)$ & $10(0.0)$ & $0.05(\mathrm{r}$ \\
\hline 41 years or older at diagnosis & 180 & $56.44(10.7)$ & $10(0.0)$ & -0.08( \\
\hline Died & 230 & $66.91(16.7)$ & $4.7(3.3)$ & $0.13(\mathrm{r}$ \\
\hline
\end{tabular}


613 new cases of epilepsy were identified in adults between January 1, 2000 and December 31, 2002. Thirty-one patients (5\%) had moved out of the study population. Of the remaining 582 cases: $352(60 \%)$ remained alive and were followed for 10 years; $230(40 \%)$ died and were followed for a mean of 4.7 years (standard deviation [SD] 3.3 years). A Wilcoxon signed-rank test was used in the cohort study to test the null hypothesis that there was no significant change in WIMD decile following diagnosis. Table 3.7 summarizes the cohort population and figure 3.9 shows a graph of the change in WIMD decile.

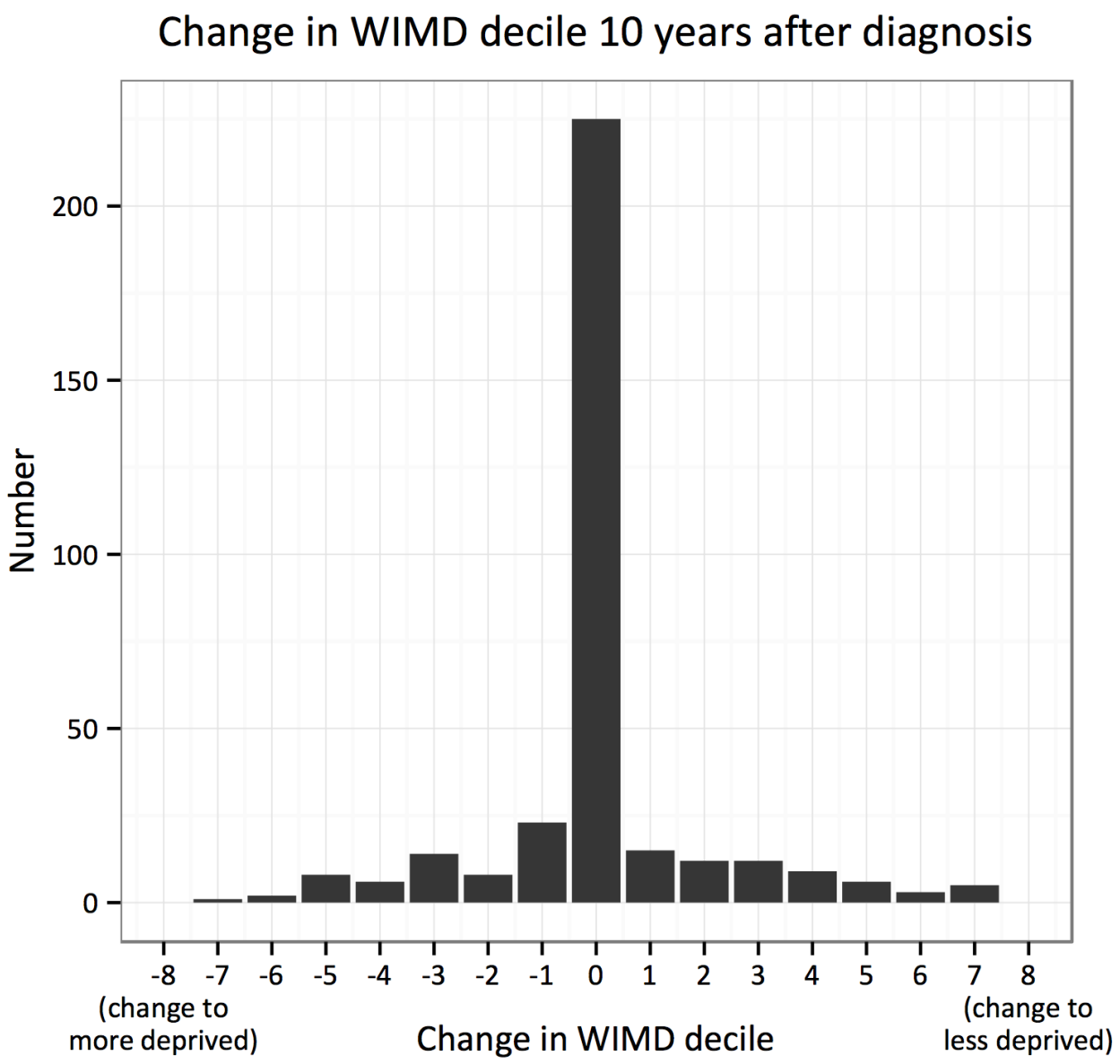

Figure 3.9: Changes in WIMD decile over 10 years for with incident epilepsy diagnosed between January 1, 2000 and December 31, 2002. Figure taken from [4] 


\subsection{Validating epilepsy status from electronic healthcare records}

The previous study used an SQL algorithm to determine an epilepsy diagnosis from GP records and compare those identified as having epilepsy with patients in the Cardiff Epilepsy database. However, it was not possible to measure the specificity of the algorithm due to a lack of a comparison cohort of people that definitely did not have epilepsy. This study aimed to validate the accuracy of algorithms using GP records to identify people with epilepsy from anonymised, linked, routinely-collected Welsh healthcare data contained within the SAIL databank.

\subsubsection{Study population}

To validate epilepsy status through the use of READ codes in the SAIL GP records, a "gold standard" dataset of patients with known epilepsy was sourced using the Swansea Epilepsy Database within Morriston Hospital ${ }^{3}$. A comparison cohort of patients without epilepsy was sourced from general neurology clinics in Morriston Hospital. There were 918 patients from the Swansea Epilepsy Database with known epilepsy (283 (29\%) generalised epilepsy, 510 (53\%) focal epilepsy, 125 (13\%) unclassifiable epilepsy and 42 (4\%) with an uncertain diagnosis), of which 100 adults and 50 children were randomly selected to form the validation set of known epilepsy. A further 300 letters from general neurology clinic letters were manually reviewed to exclude those with known epilepsy, and 100 adults and 50 children were randomly selected to form the validation set of non-epilepsy patients. The 300 person cohort was then linked to their corresponding GP records in the SAIL databank.

\subsubsection{Algorithm validation}

Three different algorithms were tested to identify people with epilepsy within the SAIL Databank. Using a READ codes within GP records, diagnosis codes for epilepsy as well as AEDS were used in the following way

- A) individuals with an epilepsy diagnosis code and two consecutive anti-epileptic drug (AED) prescription codes within 12 months of diagnosis

- B) individuals with an epilepsy diagnosis code only

- C) individuals with two consecutive AED prescription codes only.

For a full list of READ codes used to define epilepsy please see the code list in

\footnotetext{
${ }^{3}$ Data sourced by Beata Fonferko-Shadrach
} 
the appendix item 1. 145 of the 150 reference cases with epilepsy (97\%) and 143 of the 150 reference cases without epilepsy (95\%) were registered with a SAIL GP. True positive (TP) cases had a hospital diagnosis of epilepsy and were identified within SAIL as having epilepsy; true negative (TN) cases did not have epilepsy as confirmed by hospital records and were not identified as having epilepsy within SAIL; false positive (FP) cases did not have epilepsy as confirmed by hospital records and were identified as having epilepsy within SAIL; and false negative (FN) cases had a hospital diagnosis of epilepsy and were not identified as having epilepsy within SAIL. Positive predictive value (PPV) was defined as $\mathrm{TP} /(\mathrm{TP}+\mathrm{FP})$; sensitivity $\mathrm{TP} /(\mathrm{TP}$ $+\mathrm{FN})$; specificity $\mathrm{TN} /(\mathrm{TN}+\mathrm{FN})$ and false positive rate $(\mathrm{FPR})$ as $\mathrm{FN} /(\mathrm{FN}+\mathrm{TN})$. Youden's index $(\mathrm{J})$ was then calculated using sensitivity plus specificity, as a measure of the accuracy of the algorithms. J ranges from -1 to 1 ( $\mathrm{J}=1$ for a perfect test)[235]. Confidence limits were calculated using the exact binomial method. The sensitivity, specificity, positive predictive value, false positive rate and accuracy of each of the three algorithms are shown in Table 3.10. 


\begin{tabular}{|c|c|c|c|c|c|c|c|}
\hline \multicolumn{3}{|c|}{$\begin{array}{l}\text { Patients within SAIL identified as } \\
\text { having epilepsy }\end{array}$} & \multirow{2}{*}{\multicolumn{2}{|c|}{$\begin{array}{l}\text { Hospital } \\
\text { neurology } \\
\text { service } \\
\text { diagnosis of } \\
\text { epilepsy }\end{array}$}} & \multirow{3}{*}{$\begin{array}{l}\text { Positive predictive } \\
\text { value } \\
(95 \% \mathrm{Cl})\end{array}$} & \multirow{3}{*}{$\begin{array}{l}\text { Sensitivity } \\
(95 \% \mathrm{Cl})\end{array}$} & \multirow{2}{*}{$\begin{array}{l}\text { False positive } \\
\text { rate } \\
(95 \% \mathrm{Cl})\end{array}$} \\
\hline Algorithm Used & & & & & & & \\
\hline \multirow{7}{*}{$\begin{array}{l}\text { A - Epilepsy } \\
\text { diagnosis \& AED }\end{array}$} & \multirow{3}{*}{ All patients } & & Yes & No & & & \\
\hline & & Yes & 122 & 2 & \multirow[t]{2}{*}{$98 \%(94-100)$} & \multirow{2}{*}{$\begin{array}{l}84 \%(77- \\
90)\end{array}$} & \multirow[t]{2}{*}{$1 \%(0-5)$} \\
\hline & & No & 23 & 141 & & & \\
\hline & \multirow[t]{2}{*}{ Adults } & Yes & 84 & 2 & \multirow{2}{*}{$98 \%(92-100)$} & \multirow{2}{*}{$\begin{array}{l}87 \%(78- \\
93)\end{array}$} & \multirow{2}{*}{$2 \%(0-7)$} \\
\hline & & No & 13 & 96 & & & \\
\hline & \multirow[t]{2}{*}{ Children } & Yes & 38 & 0 & \multirow{2}{*}{$100 \%(91-100)$} & \multirow{2}{*}{$\begin{array}{l}79 \%(65- \\
90)\end{array}$} & \multirow{2}{*}{$0 \%(0-8)$} \\
\hline & & No & 10 & 45 & & & \\
\hline \multirow{6}{*}{$\begin{array}{l}\text { B - Epilepsy } \\
\text { diagnosis only }\end{array}$} & \multirow{2}{*}{ All patients } & Yes & 125 & 5 & \multirow{2}{*}{$96 \%(91-99)$} & \multirow{2}{*}{$\begin{array}{l}86 \%(80- \\
91)\end{array}$} & \multirow{2}{*}{$3 \%(1-8)$} \\
\hline & & No & 20 & 138 & & & \\
\hline & \multirow{2}{*}{ Adults } & Yes & 85 & 2 & \multirow{2}{*}{$98 \%(92-100)$} & \multirow{2}{*}{$\begin{array}{l}88 \%(80- \\
93)\end{array}$} & \multirow{2}{*}{$2 \%(0-7)$} \\
\hline & & No & 12 & 96 & & & \\
\hline & \multirow{2}{*}{ Children } & Yes & 40 & 3 & \multirow{2}{*}{$93 \%(81-99)$} & \multirow{2}{*}{$\begin{array}{l}83 \%(70- \\
93)\end{array}$} & \multirow{2}{*}{$7 \%(1-18)$} \\
\hline & & No & 8 & 42 & & & \\
\hline \multirow{6}{*}{ C - AED only } & \multirow{2}{*}{ All patients } & Yes & 133 & 39 & \multirow{2}{*}{$77 \%(70-83)$} & \multirow{2}{*}{$\begin{array}{l}92 \%(86- \\
96)\end{array}$} & \\
\hline & & No & 12 & 104 & & & $27 \%(20-35)$ \\
\hline & Adulte & Yes & 91 & 38 & $71 \%(63-78)$ & 94\% (87- & $39 \%(30-19)$ \\
\hline & Adults & No & 6 & 60 & $11 \%(03-/ 8)$ & 98) & $39 \%(30-49)$ \\
\hline & & Yes & 42 & 1 & Q8\% (94_100) & $88 \%(75-$ & $2 \%(0-12)$ \\
\hline & children & No & 6 & 44 & $98 \%(94-100)$ & 95) & \\
\hline
\end{tabular}

Figure 3.10: The accuracy of algorithms $\mathrm{A}, \mathrm{B}$ and $\mathrm{C}$ in being able to determine epilepsy status from GP reco 
The results show that anonymised GP records can be used to accurately identify patients with epilepsy diagnosed by a hospital specialist in the UK. Since maximizing specificity was most important, while aiming to keep sensitivity as high as possible, algorithm A (specificity 99\%, sensitivity 84\%) is best suited to identify epilepsy from GP records, where algorithm C could be exclusively used for children(specificity 98\%, sensitivity 98\%). Algorithm B shows that using a diagnosis code for epilepsy alone also achieves a high level of accuracy in adults (specificity 98\%, sensitivity 88\%), which is a $1 \%$ increase in specificity over algorithm $\mathrm{A}$, but when combining adult and children it has lower specificity than algorithms A and $\mathrm{C}$ respectively. These results compare well to other epilepsy validation studies conducted in Australian, Italian and American healthcare systems that report similar accuracy (specificity 100\%, 99.8\%, $94 \%$ and sensitivity $85.9 \%, 81 \%, 82 \%$ ) [236-238], and this study is the first epilepsy validation study using gold standard patient records accuracy in the UK.

There is a clear difference in epilepsy reporting in GP records between adults and children. It appears that GPs record a diagnosis code for a lower proportion of children than adults, resulting in only $79 \%$ sensitivity for children using algorithm A, but this is likely due to the many years required to determine a clear diagnosis of epilepsy in children. There is a large difference in specificity between adults and children for algorithm C (61\%-98\%) where it is likely conditions other than epilepsy in adults (e.g. migraine, mental health disorders and neuropathic pain) are classified as having epilepsy by the algorithm, but AEDs are rarely prescribed for anything other than epilepsy in children in the UK [239].

There was little difference in performance between algorithms A and B. Algorithm A (epilepsy + a repeat prescription) had slightly higher specificity than algorithm B (epilepsy diagnosis only) and algorithm A had slightly higher sensitivity, but their overall accuracy was comparable as seen by their Youden's Index measurement. From this study, it seems that GP diagnosis codes for epilepsy could be used on their own to identify people with epilepsy form GP records in the UK, which can be explained in that an epilepsy diagnosis should be made in secondary care by a specialist in the UK, and then recorded by GPs in GP records [240]. 


\subsection{Educational attainment of children born to mothers with epilepsy}

There are currently many AEDs used for seizure control, but some methods of seizure control during pregnancy can have effects on the unborn child. Valproate is the most effective drug for treating genetic generalized epilepsy,[26] but recent prospective psychometric studies have demonstrated cognitive impairment and neurodevelopmental disorders in 30-40\% of children exposed to valproate inutero, [241, 242] as well as a significant decrease in intelligence quotient(IQ) [27, 243]. Women with epilepsy who have satisfactory control with valproate and are planning a family therefore have a difficult decision to make. In the United Kingdom the Medicines and Healthcare Products Regulatory Agency (MHRA), issued stringent guidance for all clinicians prescribing valproate to women of child-bearing potential in 2015. An International League Against Epilepsy (ILAE) task force made seven recommendations, the first of which is where possible, valproate should be avoided in women of childbearing potential. Women with epilepsy who are taking AEDs are presently advised to continue them throughout pregnancy, primarily because of the risks of convulsive seizures to mother and her unborn child.

To be able to counsel mothers adequately about the risks of uncontrolled seizures during pregnancy and cognitive outcomes for their children, it is important to know whether the psychometric differences demonstrated in research conditions translate to children in the community. This study was conducted to investigate the effect of AED exposure inutero on the educational attainment of children born to mothers with epilepsy using anonymised, routinely-collected healthcare records and the results of a standard national educational assessment.

\subsubsection{Cohort selection}

The Child Health dataset in the SAIL databank was used to select encrypted identifiers

for children as well as a linked ID to the mother. Gestational age, maternal age were also extracted as covariates used for control matching between mothers with epilepsy at time of birth and those without. All fields in the Child Health dataset are given in table 3.8. 
Table 3.8: SAILCHDV.CHILD is a table in the SAIL Databank that contains birth records and relates each child's NHS number to their mother.

\begin{tabular}{ll}
\hline Field Name & Description \\
\hline CHILD_ID_E & Internal child ID \\
ALF_E & Encrypted NHS number of child \\
MAT_ALF_E & Encrypted NHS number of mother \\
MAT_WOB & Week of Birth of mother \\
WOB & Week of Birth of child \\
BIRTH_WEIGHT & Weight of the child at birth. \\
BIRTH_WEIGHT_CAT & Derived variable. Classes for birth weights. \\
BIRTH_TM & Time of birth \\
GNDR_CD & Sex of child \\
APGAR_1 & APGAR score taken at 1 minute \\
APGAR_2 & APGAR score taken at 5 minutes \\
GEST_AGE & Best estimate of gestation at time of delivery \\
TOT_BIRTH_NUM & Number of deliveries for multiple births \\
BIRTH_ORDER & Order of deliveries for multiple births, by ALF_E \\
MAT_AGE & Age of mother in years at delivery \\
PROV_SITE_CD & Hospital provider site code \\
STILLBIRTH_FLG & Stillbirth flagged only in case of stilbirth \\
DOD & Date of death of child \\
LHB_CD & Local healthboard code \\
DEL_CD & Delivery code indicating type of delivery \\
LABOUR_ONSET_CD & Method of labour onset \\
MOTHER_CARE_CD & Type of maternity care allocated for mother \\
BREASTFEED_BIRTH_FLG & Breastfeeding at birth \\
BREASTFEED_8_WKS_FLG & Breastfeeding at 8 weeks \\
LSOA_CD & Lower Super Output Area containing mother's address \\
\hline
\end{tabular}

For each birth record, the mother's data was linked to their social deprivation as described in the social deprivation study earlier in this chapter. WIMD quintiles at the time birth were used as an additional covariate during the control matching procedure. These data were then linked to the GP dataset in SAIL using algorithm A from the epilepsy validation study (epilepsy diagnosis + repeat AED prescription) to determine if the mother had known epilepsy during the pregnancy. A control group was created (with 4:1 matching) matched for maternal age, week of gestational age, and WIMD decile at the time of birth between mothers who had known epilepsy during pregnancy and those that did not have epilepsy. 


\subsubsection{Education dataset}

Educational attainment data for Key Stage 1 from the Department for Children Education, Lifelong Learning and Skills (DCELLS) dataset was available in the SAIL databank between the years 2003-2008. The DCELS dataset contains attainment for children in mathematics, language (English or Welsh) and science in which each subject is awarded a level between 1 (lowest) and 3 (highest). In certain circumstances children may obtain an unclassified or working towards meaning that they do not achieve the required grade to pass the year. The core subject indicator (CSI) is defined as the proportion of children achieving a minimum standard in all three KS1 subjects, that being a level 2 or higher in each subject. Given that KS1 results (taken at the age of 7) were only available within SAIL for the years 2003-2008, SAIL GP records were queried for women with epilepsy who gave birth between 1996 and 2001. Table 3.9 shows all field available within the Key Stage 1 dataset: 
Table 3.9: SAILDCELV.PRE16_KS1 is a table in the SAIL Databank contains all-Wales education data between 2003-2008 for Key Stage 1. Three subjects (Maths,Science,Engish/Welsh) as well as a Core Subject Indicator are provided to indicate the level of attainment per child

\begin{tabular}{ll}
\hline Field name & Description \\
\hline BATCH_NUM & Batch number \\
LEA & Local education Authority code \\
ESTAB_E & Encrypted educational establishment code \\
PUPIL_IRN_E & Internal pupil reference number \\
CSI & Core Subject Indicator \\
EN1 & English teacher assessment 1 \\
EN2 & English teacher assessment 2 \\
EN3 & English teacher assessment 3 \\
ENSUB & English teacher assessment subject level \\
MA1 & Maths teacher assessment 1 \\
MA2 & Maths teacher assessment 2 \\
MA3 & Maths teacher assessment 3 \\
MASUB & Maths teacher assessment subject level \\
SC1 & Science teacher assessment 1 \\
SC2 & Science teacher assessment 2 \\
SC3 & Science teacher assessment 3 \\
SC4 & Science teacher assessment 4 \\
SCSUB & Science teacher assessment subject level \\
CY1 & Welsh teacher assessment 1 \\
CY2 & Welsh teacher assessment 2 \\
CY3 & Welsh teacher assessment 3 \\
CYSUB & Welsh teacher assessment subject level \\
YEAR & Census year \\
URN & Internal school reference number \\
NEWBES & Pupils from non English/Welsh education system \\
\hline &
\end{tabular}

Each child who was born to mothers with known epilepsy during pregnancy was then linked to their education data and compared to those children not born to mothers with epilepsy. Figure 3.11 shows each step of the cohort ascertainment and linkage: 

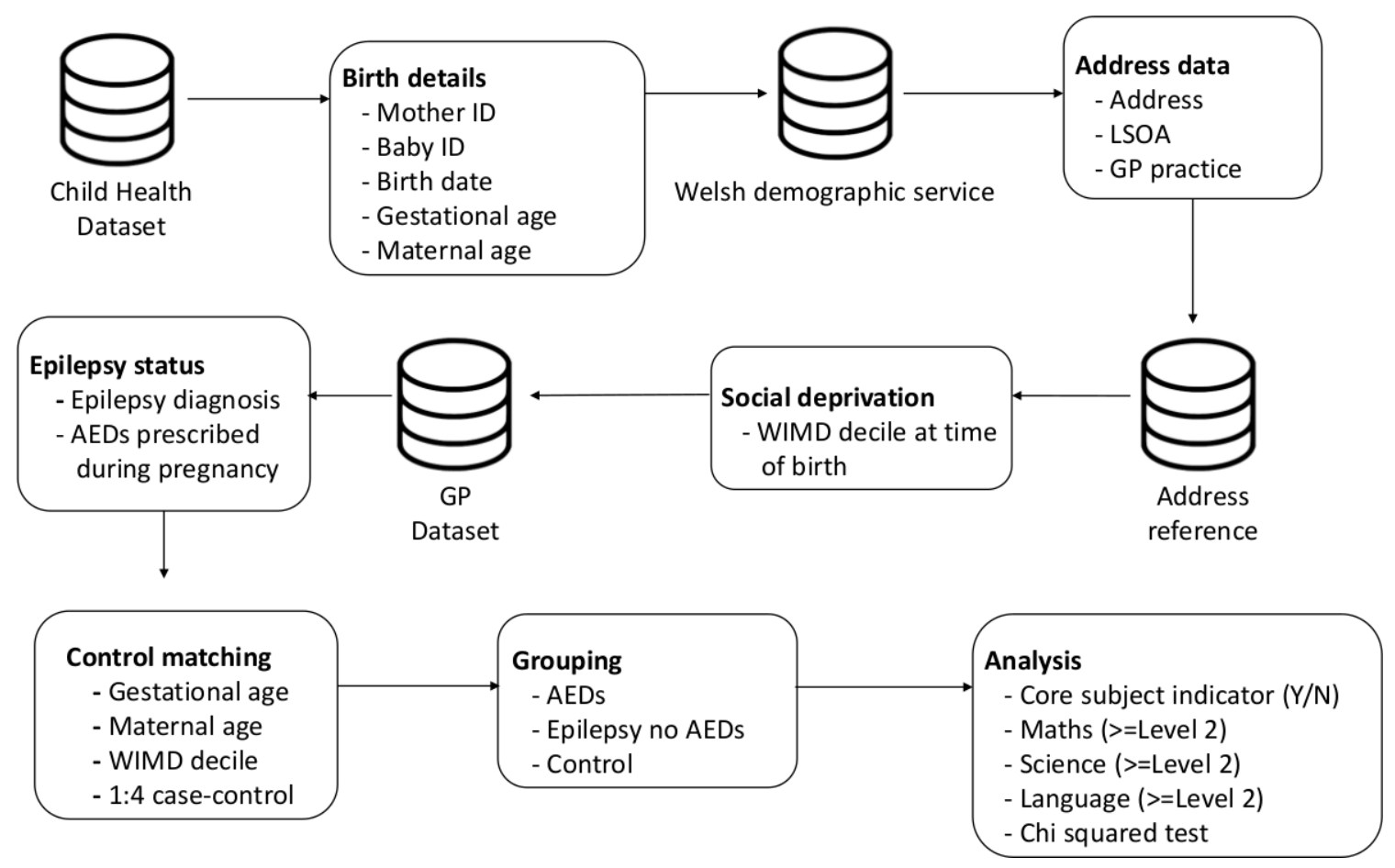

Figure 3.11: Flow chart of cohort ascertainment. 4 datasets were queried: General Practice, ONS Births, Welsh Demographic Service and Welsh Education Dataset.

\subsubsection{Results}

A total of 440 children were identified with KS1 results available between 2003 and 2008 who had mothers with epilepsy diagnosed before their pregnancy, and the mothers were stratified into five groups based on AED prescription during pregnancy (carbamazepine, lamotrigine, sodium valproate, multiple AEDs or no AEDs prescription) - see table 3.12. Only prescription information was available, but it is not expected that adherence differs across different AED prescriptions.

The proportion of children in each group achieving at least a level 2 in each subject is shown in figure 3.13 . 


\begin{tabular}{|c|c|c|c|c|c|c|c|}
\hline & COHORT & N & Male (\%) & $\begin{array}{l}\text { N children w/ } \\
\text { epilepsy }\end{array}$ & $\begin{array}{l}\text { Mean birth weight / kg } \\
(\mathrm{sd}, \mathrm{p} \text {-value*) }\end{array}$ & $\begin{array}{l}\text { Mean maternal age / } \\
\text { years } \\
\text { (sd, p-value*) }\end{array}$ & $\begin{array}{l}\text { Mean ges } \\
\text { / weeks } \\
\text { (sd, p-val }\end{array}$ \\
\hline \multirow{7}{*}{$\begin{array}{l}\text { Mothers with } \\
\text { epilepsy }\end{array}$} & Carbamazepine & 84 & $43(51)$ & 2 & $3.17(0.69,0.03)$ & $28.51(5.44,0.02)$ & 38.71 \\
\hline & Lamotrigine & 24 & $10(42)$ & 1 & $3.38(0.62,0.68)$ & $24.75(5.29,0.09)$ & 38.72 \\
\hline & No drug & 178 & $95(53)$ & 9 & $3.28(0.54,0.25)$ & $26.39(5.53,0.59)$ & 39.31 \\
\hline & Sodium valproate & 115 & $58(50)$ & 7 & $3.32(0.53,0.86)$ & $25.75(5.62,0.10)$ & 39.11 \\
\hline & Polytherapy & 39 & $20(51)$ & 2 & $3.16(0.69,0.14)$ & $27.84(6.03,0.21)$ & 38.2 \\
\hline & $\begin{array}{l}\text { With } \\
\text { valproate }\end{array}$ & 20 & $12(60)$ & 0 & $3.23(0.57,0.3)$ & $26.95(5.58,0.8)$ & 38 \\
\hline & With other & 19 & $8(42)$ & 2 & $3.08(0.81,0.13)$ & $28.78(6.49,0.16)$ & 37.7 \\
\hline Control group & & 1,756 & $889(51)$ & 0 & $3.33(0.55,1.00)$ & $26.43(5.49,1.00)$ & 39.05 \\
\hline All Wales & & 159,849 & $82,049(51)$ & 0 & $3.37(0.58,0.02)$ & $27.42,(5.81,<0.001)$ & $39.27,(2$ \\
\hline
\end{tabular}

Figure 3.12: Descriptive statistics of the study cohort. The control group comprised of a 1:4 match on materi Welsh Index of Multiple Deprivation (WIMD) quintile. WIMD quintiles are a measure of deprivation (see me the most deprived and quintile 5 being the least deprived. sd=standard deviation. ${ }^{*}$ p-values are for comparisons the "Mothers with Epilepsy" group with the control group. Table taken from [3] 
Key Stage 1 Educational Attainment
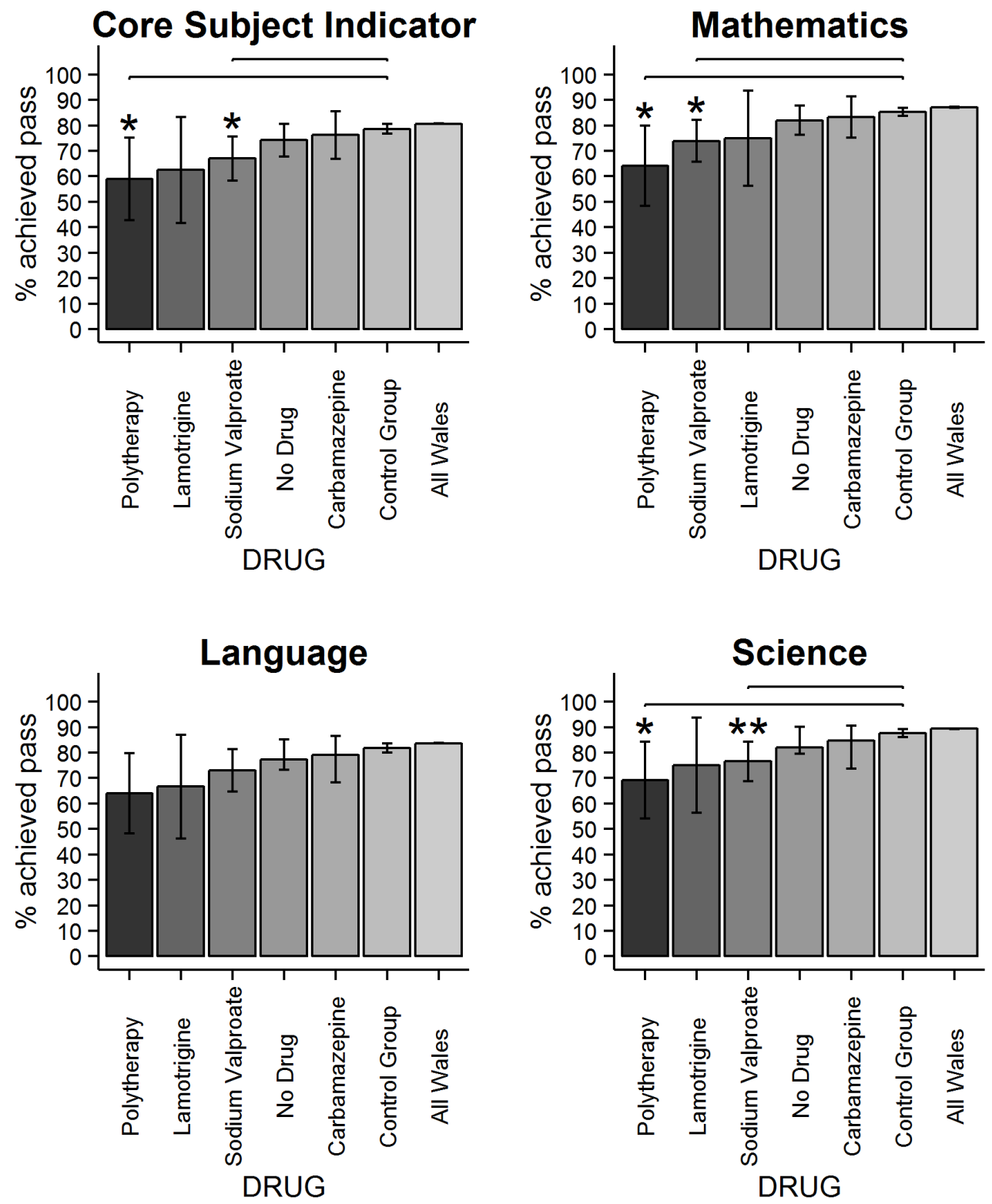

Figure 3.13: Key Stage 1 results stratified by subject and study groups. Each group was compared to the matched control group. Significant differences in attainment $(*$ $\mathrm{p} ; \mathbf{0 . 0 5}, * * \mathrm{p} ; \mathbf{0 . 0 0 5})$ between each group and the matched control are shown. The p-values have been Bonferroni corrected for multiple testing (see Methods section). The All Wales group is shown as a regional comparator only and not used to test for significance. Figure taken from [3]

These results show that children born to mothers with epilepsy being prescribed sodium valproate during pregnancy have a significantly lower level of achievement in KS1 tests across all indicators, with fewer children achieving the minimum standard when compared to the matched control group by (CSI $=-12.7 \%$ less than the control group, mathematics $=-12.1 \%$, language $=-10.4 \%$, science $=-12.2 \%$ ). Also fewer children born to mothers with epilepsy being prescribed multiple AEDs during 
pregnancy achieved the national standard in KS1 tests when compared to the matched control group by (CSI $=-20.7 \%$ less than the control group, mathematics $=-21.9 \%$, language $=-19.3 \%$, science $=-19.4 \%$ ). There was no significant decrease in attainment in children born to mothers with epilepsy that were not prescribed an AED during pregnancy according to their GP records. Excluding children with epilepsy and mothers who were recorded as smoking during pregnancy did not change the significance of these results.

\subsection{Chapter summary}

This chapter has presented various epidemiological studies in epilepsy using linked, anonymized healthcare data in the SAIL Databank. A study of GP coding of epilepsy was undertaken by comparing epilepsy diagnoses in GP records held in the SAIL databank to a gold standard dataset in Morriston hospital of patients with and without an epilepsy. The results showed that using a repeat AED prescription as well as an epilepsy diagnosis code is important in capturing epilepsy patients with high specificity, while also maintaining good sensitivity. Using this algorithm the incidence and prevalence of epilepsy in Wales was linked to social deprivation using the Welsh Index of Multiple Deprivation and showed that in more deprived areas there is both a higher incidence and prevalence of epilepsy. In a follow up study of newly diagnosed epilepsy patients, there appeared to be no increase in social deprivation leading to the conclusion that social deprivation in epilepsy is due to social causation rather than social drift. Finally, the effects of AEDs prescribed during pregnancy on children's Key Stage 1 educational attainment was explored. Children born to mothers that were prescribed sodium valproate or multiple AEDs in combination perform worse than a control group and has important cognitive outcomes for pharmaco-exposed children. 


\section{Chapter 4}

\section{Using Natural Language Processing techniques to extract clinical information from unstructured text}

This chapter aims to explore the potential of extracting rich information from epilepsy clinic letters using NLP techniques. The motivation for extracting information from clinic letters when routinely collected information is already available for research purposes, is that the information available is often limited in detail. For example, in all of the studies presented in the previous chapter they each lack specific epilepsy and seizure type, dosage details for prescribed drugs as well as finer details such as results from EEG and MRI scans. These data are available in other sources of information, namely free texts in healthcare settings. Manually reading through clinic letters to obtain rich information is time consuming, and so an automated method would be desirable to extract this data. The results in this chapter present an NLP method to extract rich epilepsy information from clinic letters stored in Morriston hospital and the Swansea Epilepsy Database.

\subsection{Clinic letters}

The Swansea Epilepsy database was used to source patients with epilepsy that had clinic letters written by epilepsy specialists at Morriston hospital. Such letters contain very detailed information regarding a patient's epilepsy such as seizure type, seizure frequency and results of examinations and investigations, and even contains information where a patient experiences symptoms similar to epilepsy that is in fact 
due to non-epileptic attack disorder. Permission to use clinic letters from the Swansea Epilepsy database was given under the condition all patient details were psuedo anonymized, where validation of any algorithm was undertaken by a clinician. 240 clinic letters were manually de-identified hospital clinic letters and used to build and test the algorithm ${ }^{1}$. 40 letters were used for training purposes to build rule sets, and a validation set of 200 letters to test the accuracy of the algorithm. The validation set contained letters originating from various outpatient clinics (145 adult epilepsy, 37 paediatric epilepsy, and 18 general neurology), from first and follow-up appointments, and written by eight different clinicians.

\subsection{A rule based NLP approach to extract epilepsy information from clinic letters}

Two approaches were considered when developing the NLP pipeline - machine learning and manually constructing rule sets. Machine learning based approaches require vast amounts of training data for the algorithm to achieve high accuracy where rule sets can take advantage of human knowledge when constructing rules. Given that only 240 letters were available for this study, this limitation was considered when deciding between a rule based and machine learning based NLP approach to analyse these letters. Due to the very large training datasets required for machine learning purposes (tens of thousands), a rule based approach in which human knowledge could be quickly built into logical rules and processed by a computer program was favoured. Therefore a rule based NLP approach was used to build an epilepsy clinical extraction pipeline that could capture data within epilepsy clinic letters.

\subsubsection{The General Architecture for Text Engineering}

The General Architecture for Text Engineering (GATE) framework was used to build a rule based NLP pipeline. Two open source applications freely available and configured for GATE were used - the biomedical named entity linking pipeline (Bio-YODIE plugin) and the South London and Maudsley medication application (SLaM). The main focus was to map clinical terms found in text to the Unified Medical Language System (UMLS) concepts so that a structured dataset could be constructed, much like the datasets that exist within the SAIL Databank. The ANNIE pipeline was used for basic POS tagging and sentence boundary detection, and the JAPE scripting language was used to program various rule sets using items of information tagged using Bio-YODIE, SLaM, ANNIE as well as custom dictionaries defined to supplement

\footnotetext{
${ }^{1}$ De-identification performed by Beata Fonferko-Shadrach
} 
these plugins. Figure 4.1 shows a flow chart of the various pipeline components:

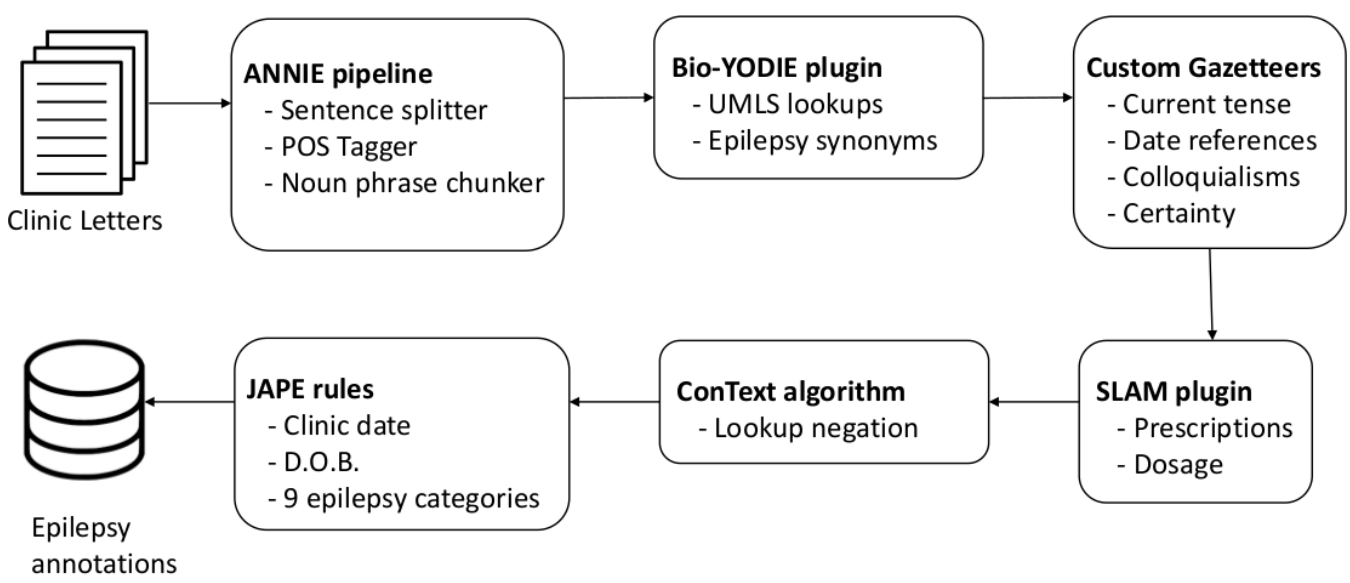

Figure 4.1: Overview of the GATE pipeline and the various components used to generate annotations

A list of predefined categories specified by a neurologist ${ }^{2}$ formed the basis of the important information to be extracted from the clinic letters:

\footnotetext{
${ }^{2}$ List provided by Dr Owen Pickrell
} 
Table 4.1: Definitions of each category intended to be extracted

\begin{tabular}{|c|c|}
\hline Category & Details \\
\hline Clinic date & The date the patient visited the clinic. \\
\hline Date of Birth & The patient's date of birth. \\
\hline Epilepsy & Items of information which confirmed a diagnosis of epilepsy e.g. \\
\hline diagnosis & $\begin{array}{l}\text { "this lady has a diagnosis of focal epilepsy" or "... has recurrent } \\
\text { unprovoked generalised tonic-clonic seizures". Frequently there is } \\
\text { diagnostic uncertainty in epilepsy clinic letters e.g. "this lady probably } \\
\text { has frontal-lobe epilepsy" or "I am uncertain whether the blackouts are } \\
\text { epileptic"; and so we defined five levels of certainty ( } 1=\text { no diagnosis, } \\
2=\text { unlikely, } 3=\text { uncertain, } 4=\text { likely, } 5=\text { definite) to each information item } \\
\text { associated with an epilepsy diagnosis. We specified that the epilepsy } \\
\text { diagnosis must be attributable to the patient (e.g. not a family member); } \\
\text { and did not include items of information that described epilepsy clinic } \\
\text { attendance, or a discussion about epilepsy in general, as confirmation of } \\
\text { an epilepsy diagnosis. }\end{array}$ \\
\hline Epilepsy type & $\begin{array}{l}\text { Whether the patient had focal or generalised epilepsy or an epilepsy } \\
\text { syndrome where epilepsy type could be inferred. For example generalised } \\
\text { epilepsy if the letter confirmed juvenile myoclonic epilepsy. This was } \\
\text { based on the UMLS CUI extracted with the epilepsy diagnosis information. } \\
\text { We only used explicit mentions of epilepsy types or syndromes within } \\
\text { the clinic letters, and did not use other information, such as seizure type } \\
\text { or investigation results, to infer epilepsy type. }\end{array}$ \\
\hline Seizure type & $\begin{array}{l}\text { Specific seizure types e.g. "focal motor seizures" or "absence seizures". } \\
\text { Seizures types were categorized into focal seizures or generalised seizures } \\
\text { at the validation stage. }\end{array}$ \\
\hline $\begin{array}{l}\text { Seizure } \\
\text { frequency }\end{array}$ & $\begin{array}{l}\text { The number of seizures in a specific time period e.g. "two seizures per } \\
\text { day", "seven seizures in a year", or "seizure free since last seen in clinic." }\end{array}$ \\
\hline Medication & $\begin{array}{l}\text { An identifiable drug name with a quantity and frequency e.g. } \\
\text { "Lamotrigine } 250 \mathrm{mg} \text { bd". }\end{array}$ \\
\hline Investigations & $\begin{array}{l}\text { The type of investigation and classification of results (normal or } \\
\text { abnormal). UMLS CUI codes were used to assign a normal / abnormal } \\
\text { value to investigation results, using the simplified abnormal outcomes } \\
\text { gazetteers. We categorised the investigation results into CT, MRI, and } \\
\text { EEG results at the validation stage. }\end{array}$ \\
\hline
\end{tabular}

\subsubsection{Defining rules}

The following sections demonstrates the specifics of how the rules for each category were built using the components in 4.1. 


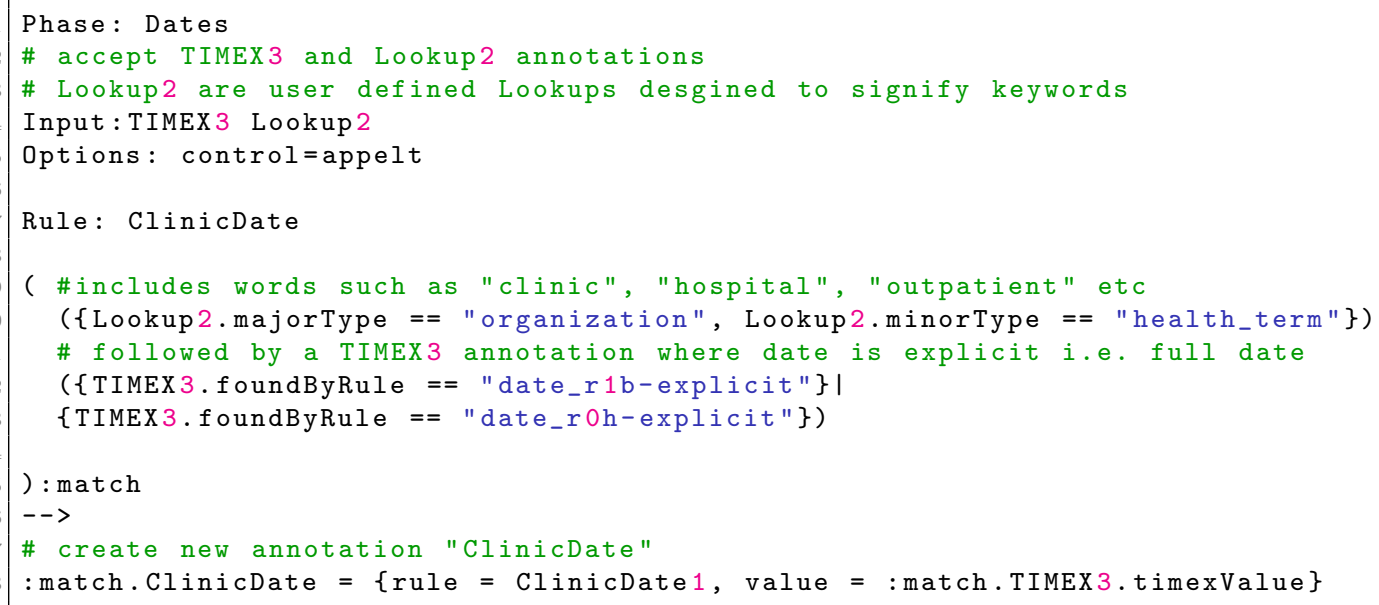

Figure 4.2: JAPE script to obtain clinic datae given an input of TIMEX3 and customs annotations relating to clinic visits (LOOKUP2)

\subsubsection{Clinic date and date of birth}

Within each letter there were various dates pertaining to different items of information such as referring to previous clinic visits, date of scans and prescriptions, as well as clinic date, date of birth and date the letter was typed up by administrative staff. The TIMEX plugin available within GATE was used to extract dates written in a variety of ways (01/01/2001 or 1st of January 2001 etc.) that were also found within the context of words/phrases suggesting clinic visits defined by custom gazetteers. The JAPE script in figure 4.2 was used to extract clinic dates.

Similarly date of birth was captured by combining TIMEX3 annotations that specify full dates i.e. day/month/year with strings such as "D.O.B", "DOB:" and "Date of birth".

\subsubsection{Epilepsy diagnosis, epilepsy type and seizure type}

Rules were built to capture phrases related to an epilepsy diagnosis attributed to a patient. Some sample phrases, where only the first two phrases would be considered to have a confirmation of epilepsy using the algorithm developed in this chapter are:

I suspect he has generalized epilepsy

She was diagnosed with focal epilepsy She doesn't have epilepsy, but has non-epileptic attacks I saw this gentleman regarding epilepsy

The first step was to identify words within phrases that indicate a mention of 


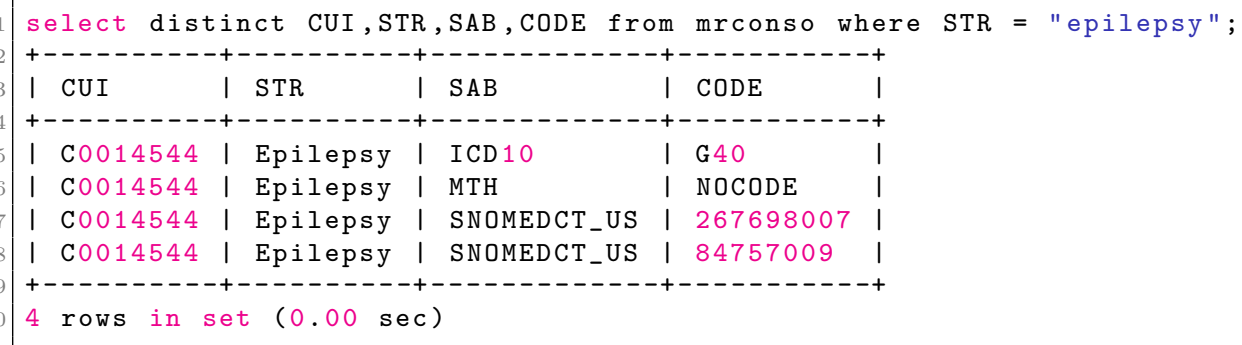

Figure 4.3: MySQL script used to query the UMLS RRF files. The table MRCONSO contains a list of all CUIs that encompass various different coding systems such as ICD 10 and SNOMED CT. By searching the STR column for the word epilepsy, the corresponding CUI is given

epilepsy. The Bio-YODIE plugin was used to map any term found in a document to a medical concept as part of the UMLS ontology, defined as a Lookup. To look specifically at epilepsy concepts, a gazetteer of epilepsy terms was built by specifying CUI codes relating to epilepsy and used to filter all Lookups within a document. Using an installation of the UMLS Metathesaurus Rich Release Format (RRF) files https://www.nlm.nih.gov/research/umls/licensedcontent/umlskn owledgesources.html the following MySQL scripts were used to query the UMLS relationship datasets for sub codes of epilepsy:

The mysql script in figure 4.3 finds all possible CUIs for the description "epilepsy" of which there is one CUI (C0014544) that unifies other existing coding systems that also describe epilepsy. This CUI was then used in script 4.4 to find child codes, or sub codes of epilepsy using the relationship file MRREL:

The script example in 4.4 shows an example list, limited to 15 items (out 2532 in total) of UMLS concepts and CUIs that are children of the epilepsy CUI by linking UMLS concepts in the MRCONSO table to the MRREL relationship table. These CUIs were used to build a Flexible Gazetteer which functions as a filter for annotations produced by the Bio-YODIE plugin. Once a subset of epilepsy terms found by Bio-YODIE has been produced, the terms found require further context to form a diagnosis. Each Bio-YODIE Lookup annotation has multiple attributes, of which the following were used to write a diagnosis annotator:

- Negation (Context plugin) - if the term has a negative context i.e. does not have epilepsy

- TUI/Unique Identifier Type (UMLS) - each concept within UMLS is 


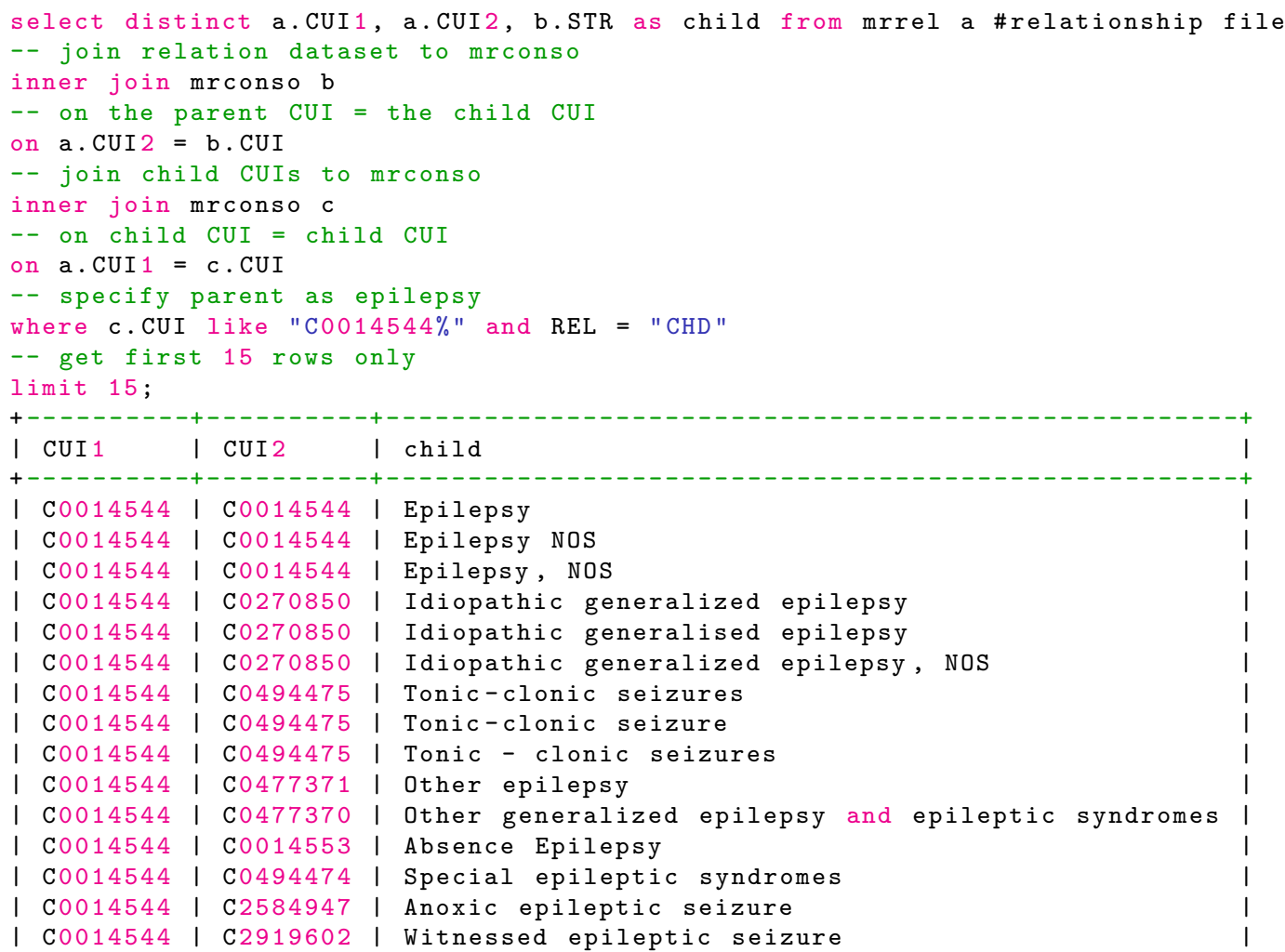

Figure 4.4: MySQL script used to query the UMLS RRF files and find all child codes of epilepsy

attributed to a semantic type e.g. "Disease or Syndrome", "Procedure", "Sign or Symptom" or "Clinical Drug" with each assigned a TUI code.

- Experiencer (Context plugin) - if the term is referenced to the primary person within the text, i.e. the patient or other such as family members

There are many components other than finding the word "epilepsy" that determine if an epilepsy diagnosis has been confirmed. Custom gazetteers were created to search for terms such as "Diagnosis:" found in structured elements of clinic letters, and custom gazetteers were also created to specify 5 levels of certainty ( 5 being most certain) of a term to differentiate phrases such as "it is doubtful that she has epilepsy" and "this is probably a case of complex partial seizures" 3 . Table 4.2 shows a list of terms and their certainty levels that was used to attach a certainty level to any Lookup:

\footnotetext{
${ }^{3}$ With assistance from Dr Owen Pickrell
} 
Table 4.2: A gazetteer of terms used to determine 5 levels of certainty attached to an epilepsy diagnosis. A confirmed diagnosis must have a value of 4 or 5 .

\begin{tabular}{llllll}
\hline Term & Level & Term & Level & Term & Level \\
\hline ruled out & 1 & possibility of & 3 & suspected & 4 \\
unlikely & 2 & $?$ & 3 & suggestive & 4 \\
doubtful & 2 & uncertain & 3 & treated as & 4 \\
doesn't & 1 & might & 3 & treating this as & 4 \\
doubt & 2 & potential & 3 & probably & 4 \\
unsure & 2 & potentially & 3 & suspicion & 4 \\
unclear & 2 & further clarification & 3 & I think & 4 \\
not convinced & 2 & further investigation & 3 & impression is & 4 \\
remote & 2 & to be confirmed & 3 & sounds like & 4 \\
improbable & 2 & to be sure & 3 & sound like & 4 \\
not likely & 2 & to see if & 3 & suspect & 4 \\
?? & 2 & could be & 3 & suspicious & 4 \\
remote possibility & 2 & to see whether & 3 & certain & 5 \\
unusual & 2 & likely & 4 & definite & 5 \\
possible & 3 & probable & 4 & are dealing with & 5 \\
\hline
\end{tabular}

The JAPE script in Figure 4.5 is one example of how potential diagnoses was captured.

The UMLS CUI codes (767 in total) from running the mysql query in Figure 4.4 were used to filter out non-epilepsy related Lookups. Figure 4.6 shows a screenshot from GATE of the features attributed to a Lookup. These features were used to determine if a Lookup was negated, its certainty level, type and it's UMLS CUI code and can be used for further downstream annotations such as only including Lookups with a certainty level greater than 3 as a confirmed diagnosis. 


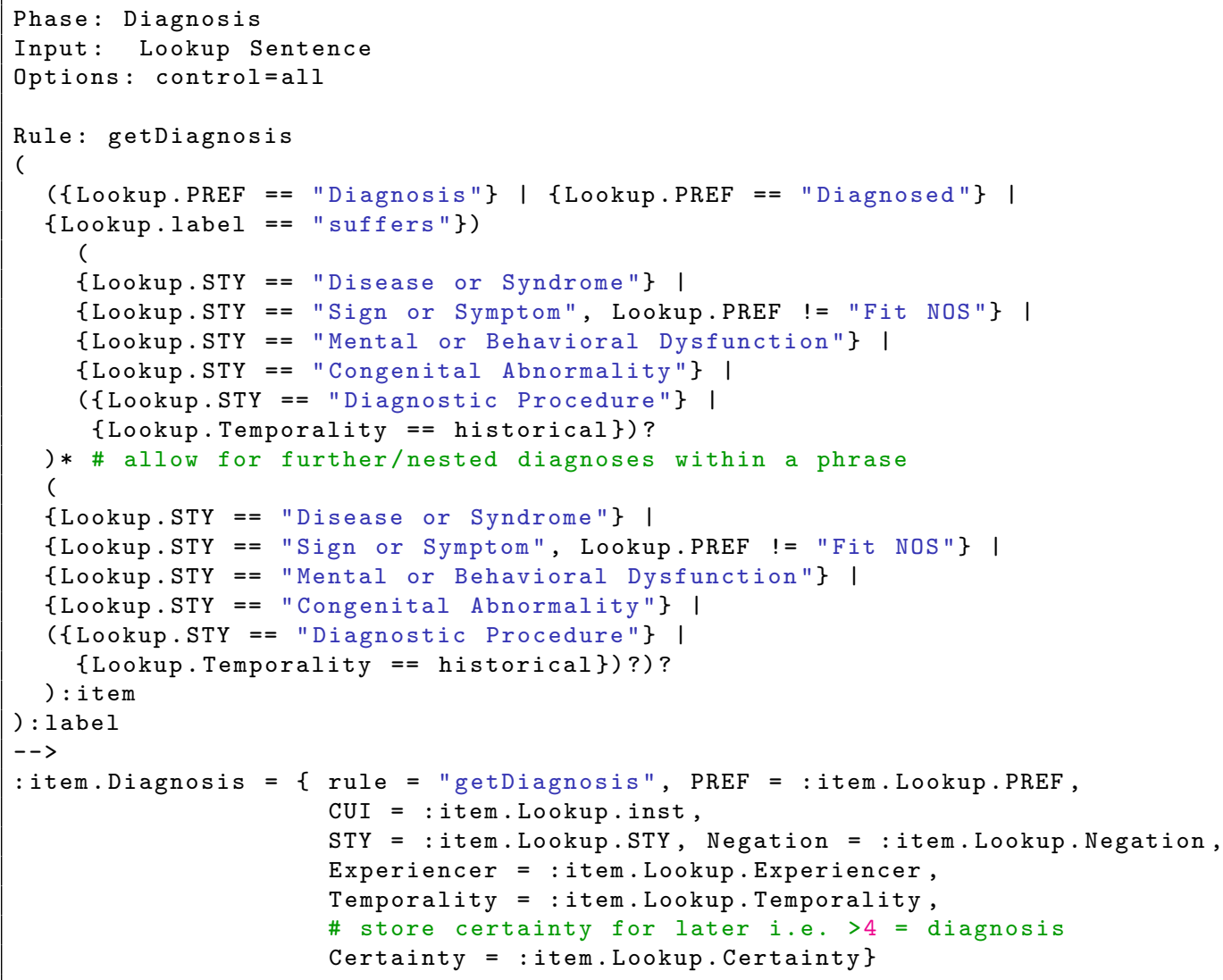

Figure 4.5: JAPE script to extract diagnosis using various contexts such negation, semantic types and certainty terms 


\begin{tabular}{|c|c|c|c|c|}
\hline $4 \quad x 24 b$ & & $\infty$ & \multicolumn{2}{|c|}{$\sqrt[3]{3}$} \\
\hline \multicolumn{3}{|l|}{ Lookup } & & $\checkmark$ \\
\hline CUIVOCABS & $\checkmark$ & MTH,OMIM,SNOMEDCT_US,CHV,HPO,DXP,WHO & $\checkmark$ & $x$ \\
\hline Certainty & $\checkmark$ & 3 & $\checkmark$ & $x$ \\
\hline Experiencer & $\checkmark$ & Patient & $\checkmark$ & $x$ \\
\hline LABELVOCABS & $\checkmark$ & MTH,OMIM,CHV,WHO,HPO & $\checkmark$ & $x$ \\
\hline Negation & $\checkmark$ & Affirmed & $\checkmark$ & $x$ \\
\hline PREF & $\checkmark$ & Psychomotor fit & $\checkmark$ & $x$ \\
\hline STY & $\checkmark$ & Disease or Syndrome & $\checkmark$ & $x$ \\
\hline TUI & $\checkmark$ & T047 & $\checkmark$ & $x$ \\
\hline Temporality & $\checkmark$ & Recent & $\checkmark$ & $x$ \\
\hline inst & $\checkmark$ & C0149958 & $\checkmark$ & $x$ \\
\hline label & $\checkmark$ & complex partial seizures & $\checkmark$ & $x$ \\
\hline language & $\checkmark$ & & $\checkmark$ & $x$ \\
\hline III & $\checkmark$ & 2169 & $\checkmark$ & $x$ \\
\hline majorType & $\checkmark$ & umls & $\checkmark$ & $x$ \\
\hline minorType & $\checkmark$ & uncased & $\checkmark$ & $x$ \\
\hline query & $\checkmark$ & complex partial seizures & $\checkmark$ & $x$ \\
\hline scCRISCUIFreq & $\checkmark$ & null & $\checkmark$ & $x$ \\
\hline scCRISCUINorm & $\checkmark$ & null & $\checkmark$ & $x$ \\
\hline scCRISLabelCUIFreq & $\checkmark$ & null & $\checkmark$ & $x$ \\
\hline scCRISLabelCUINorm & $\checkmark$ & null & $\checkmark$ & $x$ \\
\hline scCui & $\checkmark$ & 0.850042 & $\checkmark$ & $x$ \\
\hline scMeshFreqLog & $\checkmark$ & 0.0 & $\checkmark$ & $x$ \\
\hline scPageRank & $\checkmark$ & null & $\checkmark$ & $x$ \\
\hline scStringLength & $\checkmark$ & 24 & $\checkmark$ & $x$ \\
\hline scStringSimilarity & $\checkmark$ & 1.0 & $\checkmark$ & $x$ \\
\hline string & $\checkmark$ & complex partial seizures & $\checkmark$ & $x$ \\
\hline string_orig & $\checkmark$ & complex partial seizures & $\checkmark$ & $x$ \\
\hline & $\checkmark$ & & $\checkmark$ & $x$ \\
\hline
\end{tabular}

Figure 4.6: A Lookup for the phrase "possible complex partial seizures". The "Certainty" features was added through development of custom gazetteers and JAPE rules. The rest of the features come as default from the BIO-Yodie plugin in GATE, and the "Negation" feature was produced by modifying the Context plugin in GATE to add more stop words.

\subsubsection{Seizure frequency}

Seizure frequency was annotated by extracting the following items of information within text: mention of a seizure (subject to negation and certainty), number or range of seizures and the time period over which the seizures occurred. Some example phrases, of which the first four provide a measure of the number of seizures over a period of time are:

She is having 5-10 seizures per week.

He describes what are probably focal seizures. These happen at least once per day.

Since last April he has had 5 seizures.

He has had more than 20 episodes since his last visit. She was diagnosed with epilepsy after having 5 seizures.

The approach taken was that seizure frequency can be split into three parts: mention 


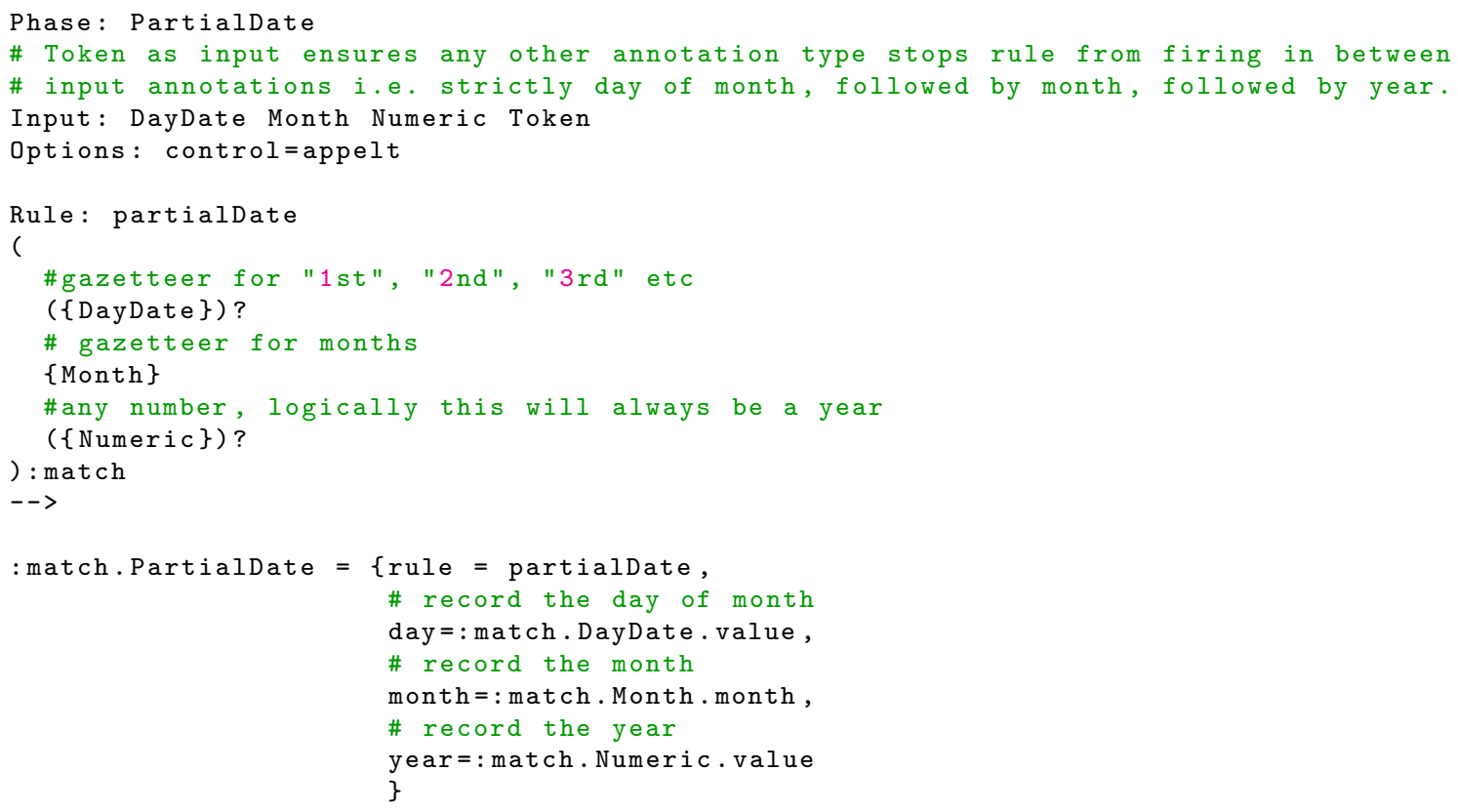

Figure 4.7: JAPE script to define all possible ways of specifying a date including partial "April 25th" and full "April 25th 1992"

of a seizure, a time period, and number of seizures. Gazetteers and JAPE rules were written to reflect these three components. Initially seizures were filtered from all Lookups identified by the BIO-Yodie plugin, but during the development of the algorithm it was found that seizure mentions aren't often specified formally e.g. "2-4 complex partial seizures per day" but rather colloquially by both patient and clinician e.g. "20 episodes since his last visit" (see example 4 above) or "15 events every morning". A custom gazetteer of terms was created to reflect this, but to preserve specificity and to distinguish "episodes" as seizures from other episodes such as episodes of depression or anxiety, a JAPE rule was written to only associate colloquial terms as seizures where a formal seizure type, such as complex partial seizure, is mentioned elsewhere in the letter.

Two approaches were taken to annotate time periods. The first was to create a JAPE rule for inferring implicit time references such as "Since April she has had around 20 seizures" where given the clinic date, a time period could be calculated. This involved capturing calendar references that span from month names i.e. April, to full date references i.e. 1st April or 1st April 2005. The Jape script in Figure 4.7 show how all of these forms are captured in a single rule using the Kleene operator for optional arguments.

The second approach was to annotate explicit mentions of a time period such as "10 
per week". Custom gazetteers for temporal terms such as "since","in the last" ,"per week", "a day" were created with attributes for each. Table 4.3 show a sample of the gazetteer used to define explicit time periods:

Table 4.3: A sample of phrases used to define explicit time periods.

\begin{tabular}{|c|c|c|c|}
\hline Item & Number & Time Unit & When \\
\hline a year & 1 & year & \\
\hline a year & 1 & year & \\
\hline b.d & 2 & day & \\
\hline b.d. & 2 & day & \\
\hline b.i.d. & 2 & day & \\
\hline $\mathrm{bd}$ & 2 & day & \\
\hline in the evening & 1 & day & evening \\
\hline every morning & 1 & day & morning \\
\hline in the morning & 1 & day & morning \\
\hline daily & 1 & week & \\
\hline every day & 1 & week & \\
\hline in a day & 1 & day & \\
\hline in a single week & 1 & day & \\
\hline in a week & 1 & week & \\
\hline per day & 1 & day & \\
\hline o.d. & 1 & day & \\
\hline at night & 1 & day & night \\
\hline a month & 1 & month & \\
\hline
\end{tabular}

The JAPE script in 4.7 and the gazetteer in Table 4.3 were used to reference various points in time and combined with seizures mentions to annotate seizure frequency as well the individual components to calculate the frequency:

\subsubsection{Medication}

The SLaM (South London and Maudsley) medication application for GATE [244] was used and modified to annotate documents with prescription information that include drug name, tablet size, unit of measurement and frequency. The SLaM application comes with custom gazetteers for drugs derived from BNF code lists, units and frequency terms which are then used as input to various JAPE rules. The drugs annotated with the SLaM application did not contain any code reference such as UMLS or READ, so the BNF gazetteer was swapped with the UMLS gazetteer 


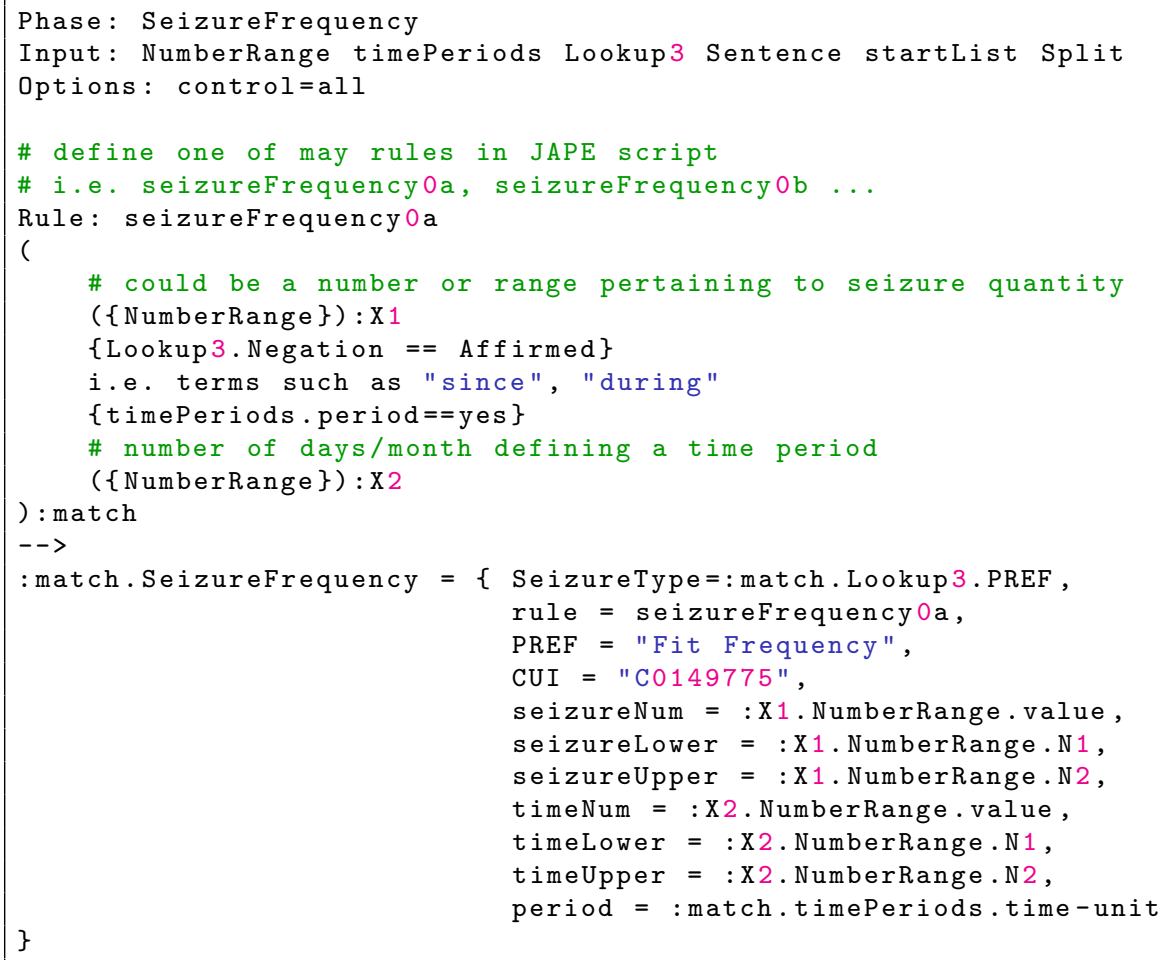

Figure 4.8: JAPE script to extract certain ways of experessing seizure frequency

used by BIO-Yodie and the JAPE scripts that came with the SLaM application were modified to accept Bio-YODIE annotations.

Other JAPE rules were also modified and supplemented with custom gazetteers to capture further details about prescriptions, such as if a prescription mention was historical, or if a prescription were to be made pending further follow up. This made it possible to select current prescriptions only. The JAPE script in figure 4.9 shows how custom gazetteers for words such as "pending", "may", "try", "previously" etc. were used to pad out prescriptions with further context:

During development of the algorithm, a common way of expressing directions to take a prescription multiple times a day was found to include times of day:

\section{$\underbrace{\text { Lamotrigine }}_{\text {Drug }} \underbrace{250 \mathrm{mg} \text { in the morning }}_{\text {Direction } \mathbf{1}}, \underbrace{200 \mathrm{mg} \text { at night }}_{\text {Direction } \mathbf{2}}$

Drug Direction $1 \quad$ Direction 2

Where both directions must be captured to sum to a daily dose of 550mg of Lamotrigine per day. The JAPE script in figure 4.10 uses Kleene operators to accommodate multiple directions when appearing consecutively without a Lookup (usually a drug given this pattern) in between them: 


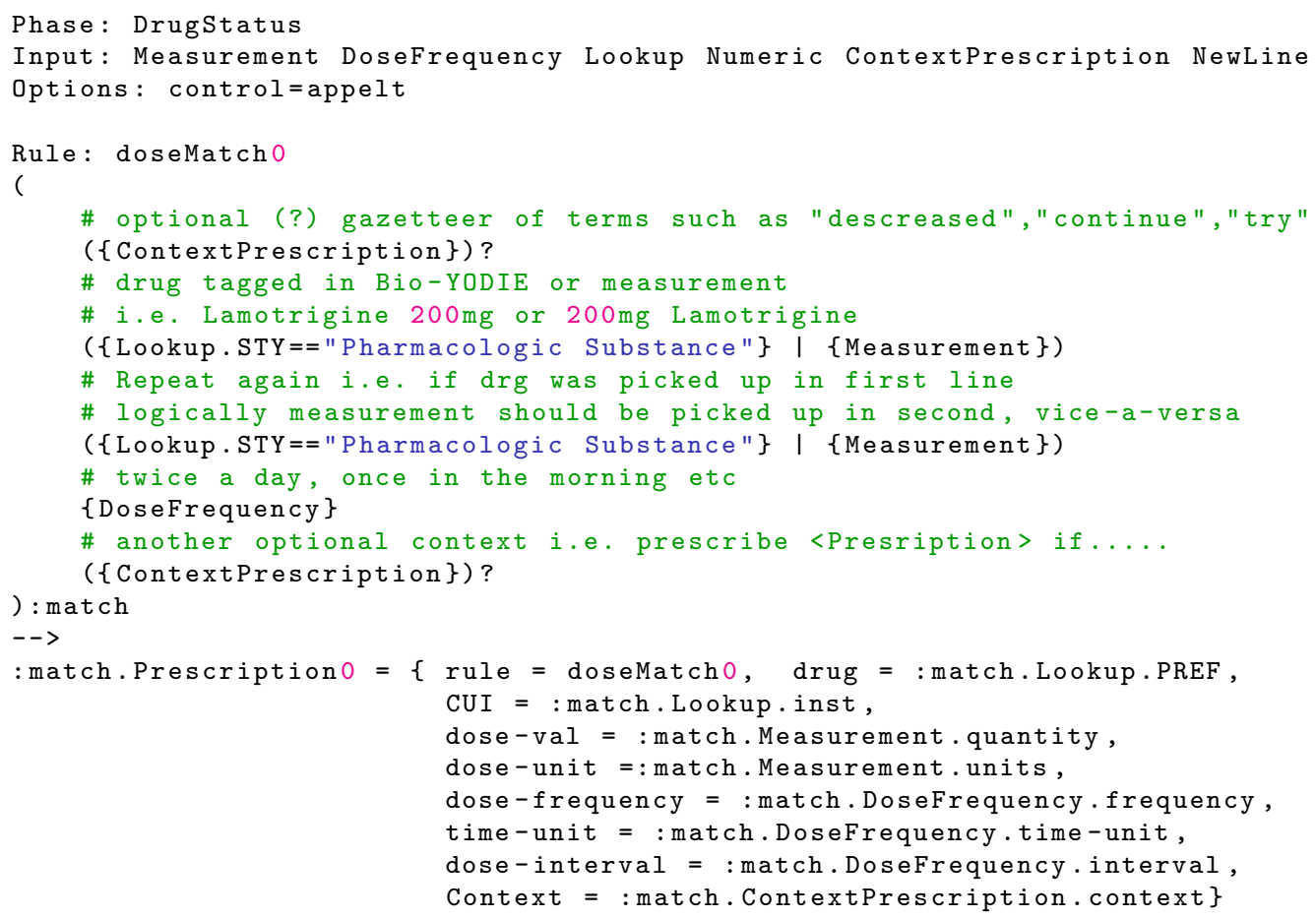

Figure 4.9: JAPE script to extract prescriptions

\subsubsection{Investigations - CT, MRI and EEG scans}

Two attempts were made to capture details of CT, MRI and EEG scans. The first attempt used CUI subsets in a similar way to how Bio-YODIE annotations were filter for epilepsy specific annotations, however terms relating to scan result were too specific to map directly to language used within clinic letters. Table 4.4 shows a sample of how UMLS concepts related to EEGs are highly specific in terms of string matching to phrases within a text: 


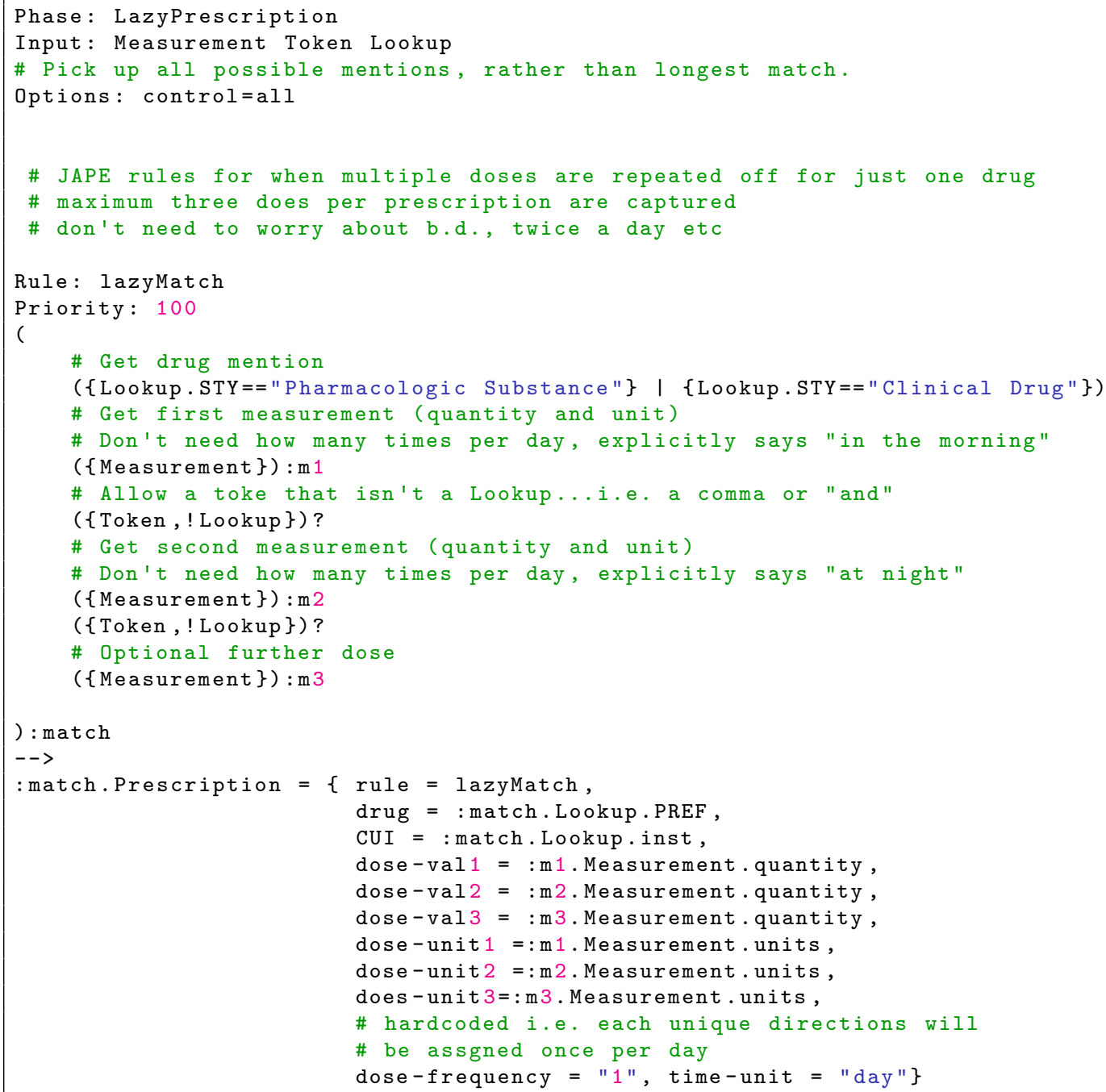

Figure 4.10: JAPE script to extract multiple prescription directions. 
Table 4.4: A sample of UMLS terms and the important information within each. UMLS terms such as these shown are difficult to map directly to text within clinical texts, where the terms of interest from within each UMLS term are much easier to map.

\begin{tabular}{ll}
\hline UMLS term & Term of interest \\
\hline EEG shows generalized, bilateral, synchronous, & symmetrical discharge \\
symmetrical discharge & \\
EEG with hyperventilation-induced focal epileptiform & focal epileptiform discharges \\
discharges & \\
EEG with hyperventilation-induced generalized & generalized \\
epileptiform discharges & discharges \\
MRI shows leukoencephalopathy with cavitation & leukoencephalopathy \\
& cavitation \\
MRI shows congenital abnormalities of the posterior & congenital abnormalities \\
fossa & \\
MRI shows short, thick corpus callosum & abnormal corpus callosum \\
Diffuse cerebral atrophy on CT and MRI & cerebral atrophy \\
Aplasia of posterior semicircular canal on CT scan & aplasia \\
Low density white matter on CT scan & low density white matter \\
\hline
\end{tabular}

In table 4.4 the UMLS terms in column 1 only get mapped to terms in text if the exact term is found, but due to the length and specificity of some terms there was low sensitivity in picking up investigations. Therefore custom gazetteers that use smaller terms categorised as normal (CUI:C0560017) or abnormal (CUI:C0151611) were produced to maximize to maximize sensitivity of investigation outcomes that preserve what is important i.e. normal or abnormal results ${ }^{4}$. Table 4.5 shows a list of terms used to identify possible investigation findings:

\footnotetext{
${ }^{4}$ with assistance from Dr Owen Pickrell
} 
Table 4.5: A list of custom terms used to indicate a possible EEG finding

\begin{tabular}{llll}
\hline Custom term & $\begin{array}{l}\text { Derived } \\
\text { UMLS } \\
\text { concept }\end{array}$ & Custom term & $\begin{array}{l}\text { Derived } \\
\text { UMLS }\end{array}$ \\
& C0151611 & normal & concept \\
\hline abnormal & C0151611 & normal EEG & C0560017 \\
abnormal EEG & C0151611 & photosensitive & C0560017 \\
abnormalities & C0151611 & photosensitive & C0151611 \\
abnormality & C0151611 & photosensitivity & $\mathrm{C} 0151611$ \\
burst suppression & $\mathrm{C} 0560017$ & polyspike & $\mathrm{C} 0151611$ \\
clear & $\mathrm{C} 0560017$ & poly-spike & $\mathrm{C} 0151611$ \\
did not capture & any & & \\
events & $\mathrm{C} 0151611$ & polyspike and wave & $\mathrm{C} 0151611$ \\
dysrhythmic & $\mathrm{C} 0560017$ & right side slowing & $\mathrm{C} 0151611$ \\
EEG normal & $\mathrm{C} 0151611$ & sharp & $\mathrm{C} 0151611$ \\
epileptic & $\mathrm{C} 0151611$ & spike & $\mathrm{C} 0151611$ \\
epileptiform & $\mathrm{C} 0151611$ & spike and wave & $\mathrm{C} 0151611$ \\
epileptogenic & $\mathrm{C} 0560017$ & spikes & $\mathrm{C} 0151611$ \\
failed to alter & $\mathrm{C} 0151611$ & spike-wave & $\mathrm{C} 0151611$ \\
focal slowing & $\mathrm{C} 0151611$ & temporal slowing & $\mathrm{C} 0151611$ \\
focus & $\mathrm{C} 0151611$ & unremarkable & $\mathrm{C} 0560017$ \\
generalised slowing & $\mathrm{C} 0151611$ & unstable & $\mathrm{C} 0151611$ \\
irregular & $\mathrm{C} 0151611$ & & \\
left side slowing & & & \\
\hline
\end{tabular}

Once terms were identified within the text, the JAPE rule in figure 4.11 was written to associate them to investigation names using strings such as "EEG", "MRI" and "CT" as long as they were found after the investigation name and within the same paragraph:

\subsubsection{Validation of Algorithm}

After developing the pipeline, 200 unseen letters were used to validate accuracy against a clinician ${ }^{5}$. For each category of information, the scope of what was expected to be extracted or not extracted was discussed with the clinician. The clinician then annotated every letter for each category, including multiple mentions from the same category. Separately, the pipeline was run against the 200 test letters and annotated

${ }^{5}$ performed by Dr Owen Pickrell 


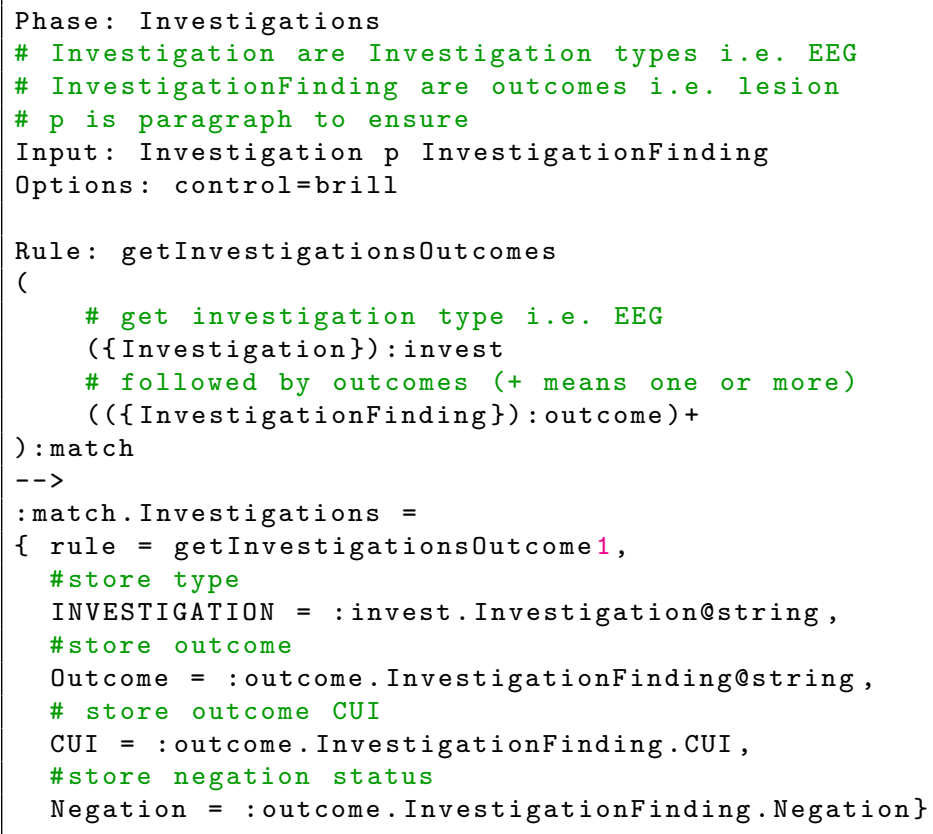

Figure 4.11: JAPE script to extract investigation outcomes

for each category and the results of the clinician and pipeline were reviewed, where all disagreements were manually reviewed.

Precision, recall and F1-score were used as measures to determine the accuracy of the pipeline and are defined as:

$$
\begin{gathered}
\text { Precision }=\frac{T P}{T P * F P} \\
\text { Recall }=\frac{T P}{T P * F N} \\
F 1 \text { score }=\frac{\text { Precision } * \text { Recall }}{\text { Precision }+ \text { Recall }}
\end{gathered}
$$

True positives were defined as both the pipeline and clinician identifying a positive finding such as confirming an epilepsy diagnosis, false positives were defined as the pipeline identifying a positive finding where the clinician did not, and false negatives were defined as the algorithm failing to identify a positive finding where the clinician was able to. To resolve any mention where there was a disagreement, either the disagreement remained after manual review, or in small proportion of cases where the clinician had made a mistake, the clinicians record was corrected. Results are given in table 4.6 where a "per item" score is based on every possible mention within the categories, and a "per letter" score assumes that identification of one true positive 
within a given category is a true positive for that category as a whole i.e. if there are 3 mentions of an epilepsy diagnosis, if the pipeline was able to identify just one, the per letter score for epilepsy diagnosis would be a true positive. 
Table 4.6: Precision, recall and F1-score are calculated across 9 epilepsy specific categories as well as clinic d approaches have been considered - the first measures the algorithm's accuracy for every mention $(\mathrm{N}=1925)$ acros in the manual review by the clinician, and the second approach aggregates results from multiple mentions pe there are multiple true mentions regarding confirmation of epilepsy in a single letter, we assign a single true posi picks up at least one of these mentions, with the same logic used to determine false positives, true negatives ar

\begin{tabular}{lllllll}
\hline Variables & \multicolumn{3}{l}{ Per item performance } & \multicolumn{2}{c}{ Per letter performance } \\
\hline & N items & Precision $\%$ & Recall $\%$ & F1 score $\%$ & N letters & Precision \% \\
Clinic Date & 191 & 98.9 & 97.4 & 98.2 & 186 & 100 \\
Date of Birth & 201 & 100 & 98 & 99 & 199 & 100 \\
Epilepsy Confirmed & 383 & 88.1 & 99 & 88.5 & 150 & 94.1 \\
Epilepsy Type & 89 & 89.9 & 79.8 & 84.5 & 70 & 91 \\
Focal Seizures & 145 & 96.2 & 69.7 & 80.8 & 69 & 96.7 \\
Generalised Seizures & 151 & 88.8 & 52.3 & 65.8 & 76 & 89.7 \\
Seizure Frequency & 153 & 86.3 & 53.6 & 66.1 & 119 & 92.2 \\
Medication & 316 & 96.1 & 94 & 95 & 157 & 98.6 \\
CT Scan & 17 & 55.6 & 58.8 & 57.1 & 16 & 76.9 \\
MRI Scan & 109 & 82.4 & 68.8 & 75 & 66 & 86.7 \\
EEG & 170 & 81.5 & 75.3 & 78.3 & 79 & 86.6 \\
All & 1925 & 90.6 & 80.8 & 85.4 & 1187 & 96.6 \\
\hline
\end{tabular}


The pipeline obtained an overall precision, recall and F1-score of 91\%, $81 \%$ and $85 \%$ on a per item basis, where high scores were obtained in prescription $(\mathrm{F} 1=95 \%)$, confirmation of an epilepsy diagnosis (93\%), epilepsy type (84\%) and presence of focal seizures (81\%). The algorithm was less accurate in identifying CT (57\%), MRI $(75 \%)$ and EEG results (78\%), seizure frequency (66\%) and generalised seizure terms $(66 \%)$ given the complexity and high variance in expressing these concepts in clinic letters. The pipeline achieved even higher overall scores for precision, recall, and F1-score $(96 \%, 87 \%, 91 \%)$ on a per letter basis, in which given how the final data is to be used for further research purposes, a decision for each category can be made accurately across measures such as epilepsy diagnosis, epilepsy type, seizure type and prescriptions.

\subsection{Chapter Summary}

A gold standard dataset of de-identified clinic letters was used to build and test an NLP pipeline, that was found to accurately extract novel information about epilepsy when compared to manual review by a clinician. The use of UMLS terminologies, in particular the ability to map findings to CUI codes can be powerful in curating structured datasets that can be linked to other routinely collected data such as GP and hospital patient records, where these data can be processed programmatically rather than via manual review. Some categories such as diagnosis of epilepsy, epilepsy type and prescriptions can be extracted with high accuracy, but some concepts such as EEG/MRI/CT investigations and seizure frequency remained difficult to extract and further improvements are necessary for further research purposes. However, the information that the pipeline can extract well would improve the richness of data such as GP records that are held within the SAIL databank.

While gold standard letters have been used, the pipeline was tested on a relatively small number of letters sourced from one Health-board with a limited number of writing styles and letter structures, therefore the generalizability of the pipeline may be limited and would benefit from a larger test set. The pipeline does not however rely on the structure of clinic letters and is designed to use free text without relying on dedicated sections in the letters. A "per item" and "per letter" score was calculated to validate both the accuracy of the pipeline, but also to validate how information within letters can be used practically for further research by summarising all items in a clinic letter and giving a decision boundary on the category as a whole. 


\section{Chapter 5}

\section{Predicting functional impact of Single Nucleotide Polymorphisms}

The aim of this chapter was to investigate the best method for indicating that a Single Nucleotide Polymorphism (SNP) is a pathogenic consideration for epilepsy/neurology phenotypes. Given that an exome contains 30-40 thousands SNPs it is important to prioritise those that may contribute to disease so that they can be studied in downstream functional validation and link to observable clinical outcomes. Various machine -learning techniques were explored to identify the most accurate method of classifying disease (pathogenic) and benign SNPs. These techniques were compared to existing prediction software when tested on a disease non-specific dataset of SNPs as well as epilepsy specific SNPs. The humvar dataset was identified from an extensive literature review to be a commonly-used training dataset for machine learning classification of SNPs. It contains over 70,000 pathogenic and benign SNPs obtained from published studies. These SNPs were used to train various machine learning algorithms which were then compared to existing prediction algorithms widely-used in the literature. A set of SNPs found in genes associated with epilepsy were also scored using the trained algorithm. All code used in this chapter can be found at the following Github repository https://github.com/arronlacey/PhDChapter5.

\section{$5.1 \quad$ Features}

An automated feature extraction pipeline was built so that for each mutation in the humvar dataset, the pipeline obtains 30 protein features which are all used to train 
and test various machine learning algorithms. The pipeline consists of bash and $\mathrm{R}$ scripts to pull data from multiple websites, public databases and derive further features with downstream processing. Figure 5.1 shows a flowchart of the main processes in the feature extraction pipeline:

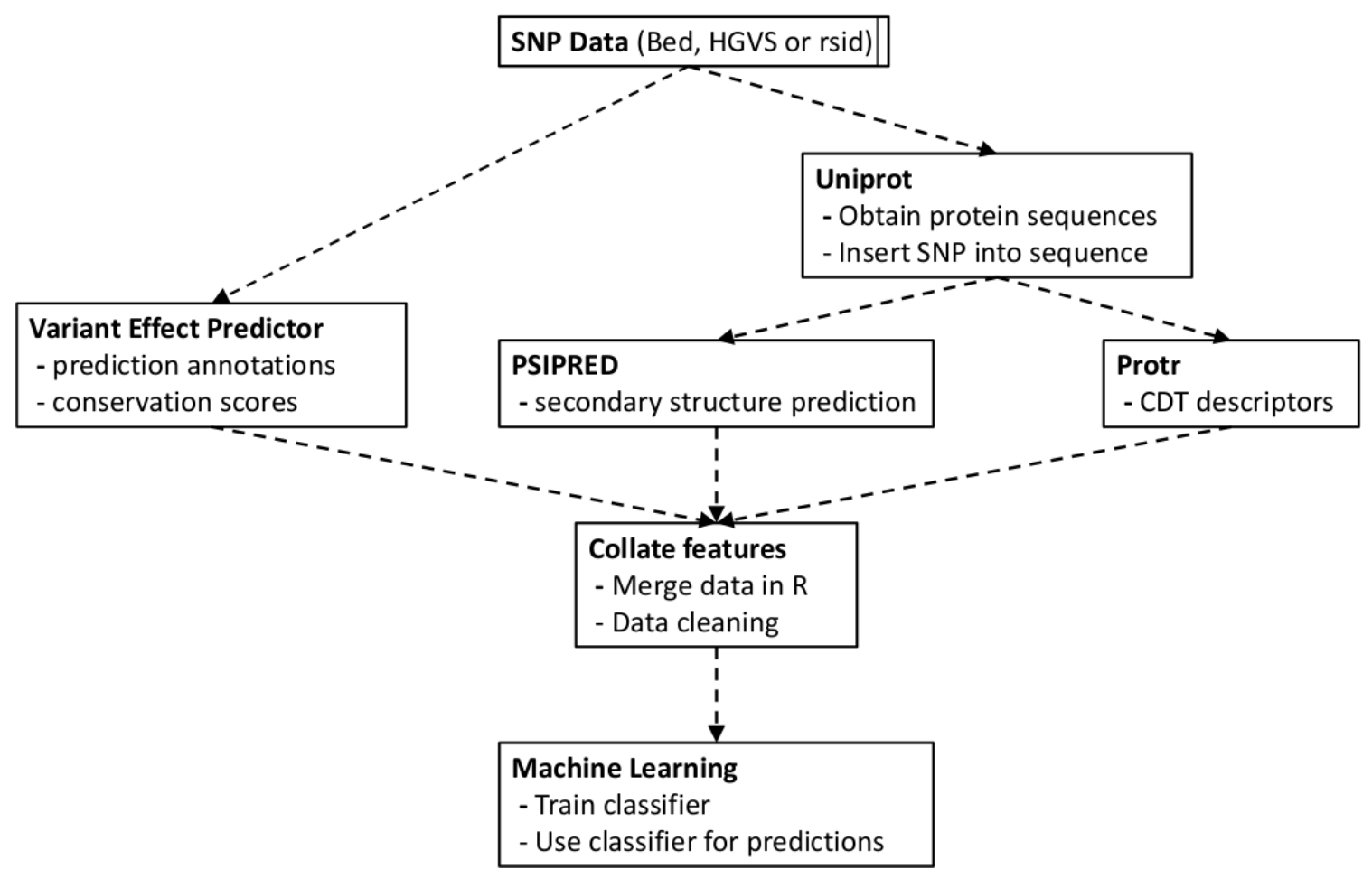

Figure 5.1: Pipeline of SNP data collection. The data is used to train a classifier that can be used to predict disease/benign status of a SNP.

The pipeline was built to accommodate chromosomal or amino acid co-ordinates in BED and HGVS formats, as well as rsid format. Sections 5.1.1 - 5.1.3 document how each process in the feature extraction pipeline was built.

\subsubsection{Variant Effect Predictor}

The humvar dataset rsids were used as input to the Variant Effect Predictor (VEP) annotating system to obtain conservation scores and existing prediction software scores for each SNP. Table 5.1 describes the 38 features collected to be used as part of the training data for machine learning. 


\begin{tabular}{|c|c|}
\hline Feature & Description \\
\hline DANN_score & Deep learning SNP prediction score [204] \\
\hline GM12878_fitCons_score & Fitness conservation score for lymphoblastiod cells [245] \\
\hline GM12878_fitCons_score_rankscore & Rankscore for lymphoblastoid cells [245] \\
\hline GenoCanyon_score_rankscore & Prediction score for non-coding function regions [246] \\
\hline H1NAhESC_fitCons_score_rankscore & Fitness conservation score for human embryonic stem cells [245] \\
\hline HUVEC_fitCons_score_rankscore & Fitness conservation score for umbilical vein epithelial cells [245] \\
\hline MetaLR_score & $\begin{array}{l}\text { SNP prediction score using } 9 \text { existing SNP software, trained with } \\
\text { logistic regression }[205]\end{array}$ \\
\hline MutationAssessor_score_rankscore & SNP prediction application for cancer variants [206] \\
\hline REVEL_score & $\begin{array}{l}\text { SNP prediction aggregation score using } 8 \text { SNP prediction software } \\
\text { for predicting pathogenicity of rare variants }[207]\end{array}$ \\
\hline fathmmNAMKL_coding_score & SNP prediction score using Hidden Markov Models [208] \\
\hline integrated_fitCons_score & Combined fitcons score \\
\hline integrated_fitCons_score_rankscore & Combined fitcons rank score \\
\hline PolyPhen_score & SNP prediction score using a Naïve Bayes classifier [196] \\
\hline SIFT_score & SNP prediction score using protein conservation methods [187] \\
\hline CADD_raw_rankscore & $\begin{array}{l}\text { Combined Annotation-Dependent Depletion SNP prediction score } \\
\text { [209] }\end{array}$ \\
\hline DANN_rankscore & DANN converted rankscore $[204]$ \\
\hline EigenNAPCNAraw_rankscore & SNP prediction score using unsuperivsed learning [247] \\
\hline FATHMM_converted_rankscore & FATHMM converted rankscore \\
\hline GERP.._RS_rankscore & Conservation score in humans based on 1,092 genomes [248] \\
\hline MetaLR_rankscore & MetaLR rankscore \\
\hline MetaSVM_rankscore & $\begin{array}{l}\text { SNP prediction score using } 9 \text { existing SNP software, trained with } \\
\text { SVM }[205]\end{array}$ \\
\hline MutationTaster_converted_rankscore & Mutation taster rankscore \\
\hline PROVEAN_score & SNP prediction in non-coding regions [249] \\
\hline REVEL_rankscore & REVEL rankscore \\
\hline SiPhy_29way_logOdds_rankscore & SIte-specific PHYlogenetic analysis [224] \\
\hline VEST3_rankscore & Variant Effect Scoring Tool $[210]$ \\
\hline fathmmNAMKL_coding_rankscore & FATHMM coding rankscore \\
\hline phastCons100way_vertebrate_rankscore & Evolutionary conservation (ranked) scores in vertabrae [223] \\
\hline phastCons20way_mammalian_rankscore & Evolutionary conservation (ranked) scores in mammals [223] \\
\hline phyloP100way_vertebrate_rankscore & Score predicting non-neutral substitution rates in vertebrae [222] \\
\hline phyloP20way_mammalian_rankscore & Score predicting non-neutral substitution rates in mammals [222] \\
\hline Reliability_index & Reliability index as calculated by SNAP2 \\
\hline GERP...NR & Conservation score in humans based on 1,092 genomes [248] \\
\hline SiPhy_29way_logOdds & SiPhy log odds score \\
\hline phastCons20way_mammalian & Evolutionary conservation scores in mammals [223] \\
\hline gnomAD & $\begin{array}{l}\text { Genome aggregation SNP frequency in population combined } \\
\text { exomes and genomes }\end{array}$ \\
\hline gnomAD_exomes & Genome aggregation SNP frequency in population in exomes \\
\hline gnomAD_genomes & Genome aggregation SNP frequency in population in genomes \\
\hline
\end{tabular}


Table 5.2: Amino acid attributes with three group classification. Each classification is given by a unique set of amino acids. Table reproduced with permission from https://cran.r-project.org/web/packages/protr/vignettes/protr.html

\begin{tabular}{|c|c|c|c|}
\hline Atrribute & Group 1 & Group 2 & Group 3 \\
\hline \multirow[t]{2}{*}{ Hydrophobicity } & Polar & Neutral & Hydrophobicity \\
\hline & $\mathrm{R}, \mathrm{K}, \mathrm{E}, \mathrm{D}, \mathrm{Q}, \mathrm{N}$ & $\begin{array}{l}\mathrm{G}, \mathrm{A}, \mathrm{S}, \mathrm{T}, \mathrm{P}, \mathrm{H}, \\
\mathrm{Y}\end{array}$ & $\begin{array}{l}\mathrm{C}, \mathrm{L}, \mathrm{V}, \mathrm{I}, \mathrm{M}, \mathrm{F}, \\
\mathrm{W}\end{array}$ \\
\hline \multirow{2}{*}{$\begin{array}{l}\text { Normalized van der } \\
\text { Waals Volume }\end{array}$} & $0-2.78$ & $2.95-4.0$ & $4.03-8.08$ \\
\hline & $\begin{array}{l}\mathrm{G}, \mathrm{A}, \mathrm{S}, \mathrm{T}, \mathrm{P}, \mathrm{D}, \\
\mathrm{C}\end{array}$ & $\mathrm{N}, \mathrm{V}, \mathrm{E}, \mathrm{Q}, \mathrm{I}, \mathrm{L}$ & $\begin{array}{l}\mathrm{M}, \mathrm{H}, \mathrm{K}, \mathrm{F}, \mathrm{R}, \mathrm{Y} \text {, } \\
\mathrm{W}\end{array}$ \\
\hline \multirow[t]{2}{*}{ Polarity } & $4.9-6.2$ & $8.0-9.2$ & $10.4-13.0$ \\
\hline & $\begin{array}{l}\mathrm{L}, \mathrm{I}, \mathrm{F}, \mathrm{W}, \mathrm{C}, \mathrm{M}, \\
\mathrm{V}, \mathrm{Y}\end{array}$ & $\mathrm{P}, \mathrm{A}, \mathrm{T}, \mathrm{G}, \mathrm{S}$ & $\begin{array}{l}\mathrm{H}, \mathrm{Q}, \mathrm{R}, \mathrm{K}, \mathrm{N}, \mathrm{E}, \\
\mathrm{D}\end{array}$ \\
\hline \multirow[t]{2}{*}{ Polarizability } & $0-1.08$ & $0.128-0.186$ & $0.219-0.409$ \\
\hline & $\mathrm{G}, \mathrm{A}, \mathrm{S}, \mathrm{D}, \mathrm{T}$ & $\begin{array}{l}\text { C, P, N, V, E, Q, } \\
\text { I, L }\end{array}$ & $\begin{array}{l}\mathrm{K}, \mathrm{M}, \mathrm{H}, \mathrm{F}, \mathrm{R}, \mathrm{Y}, \\
\mathrm{W}\end{array}$ \\
\hline \multirow[t]{2}{*}{ Charge } & Positive & Neutral & Negative \\
\hline & $\mathrm{K}, \mathrm{R}$ & $\begin{array}{l}\text { A, N, C, Q, G, H, } \\
\text { I, L, M, F, P, S, T, } \\
\text { W, Y, V }\end{array}$ & $\mathrm{D}, \mathrm{E}$ \\
\hline \multirow[t]{2}{*}{ Secondary Structure } & Helix & Strand & Coil \\
\hline & $\begin{array}{l}\mathrm{E}, \mathrm{A}, \mathrm{L}, \mathrm{M}, \mathrm{Q}, \mathrm{K}, \\
\mathrm{R}, \mathrm{H}\end{array}$ & $\begin{array}{l}\mathrm{V}, \mathrm{I}, \mathrm{Y}, \mathrm{C}, \mathrm{W}, \mathrm{F}, \\
\mathrm{T}\end{array}$ & $\mathrm{G}, \mathrm{N}, \mathrm{P}, \mathrm{S}, \mathrm{D}$ \\
\hline \multirow[t]{2}{*}{ Solvent Accessibility } & Buried & Exposed & Intermediate \\
\hline & $\begin{array}{l}\text { A, L, F, C, G, I, } \\
\text { V, W }\end{array}$ & $\mathrm{R}, \mathrm{K}, \mathrm{Q}, \mathrm{E}, \mathrm{N}, \mathrm{D}$ & $\mathrm{M}, \mathrm{S}, \mathrm{P}, \mathrm{T}, \mathrm{H}, \mathrm{Y}$ \\
\hline
\end{tabular}

\subsubsection{CTD Descriptors}

CDT (Composition/Transition/Distribution) descriptors were used to assign physiochemical attributes to each SNP [250]. The protr $\mathrm{R}$ package contains a function that calculates the global distribution of amino acid attributes classed into 3 categories as a percentage of all amino acids in a given sequence. These attributes and categories are shown in Table 5.2

The protr $\mathrm{R}$ function was modified to output the attributes and categories for every amino acid in a sequence so that data on both a wild type and a SNP could be obtained and the differences compared. The modified function is shown in Figure 5.2 


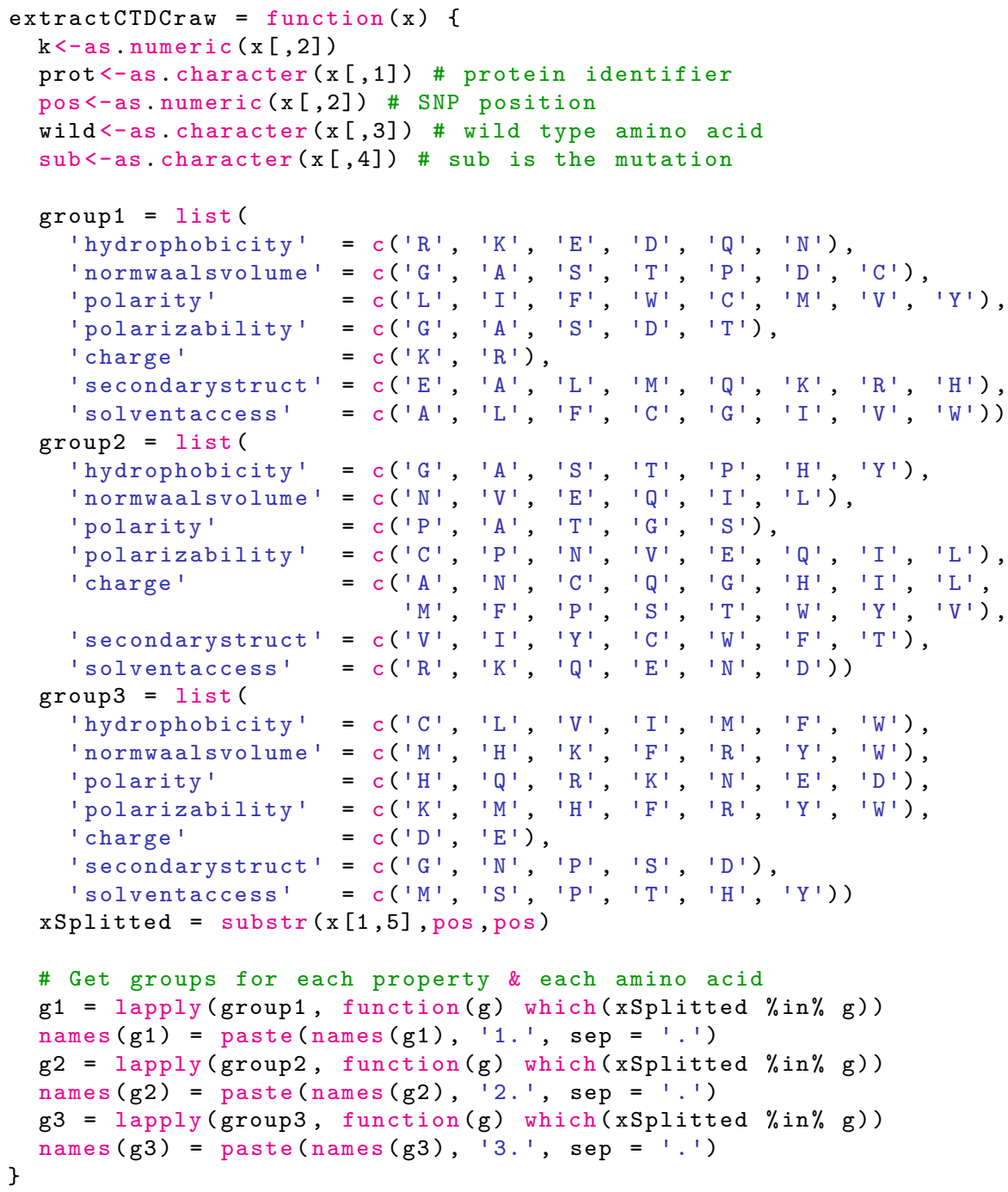

Figure 5.2: $\mathrm{R}$ function modified from the protr package to obtain $\mathrm{CTD}$ amino acid groups and categories for each position of a protein sequence 


\subsubsection{Secondary structure prediction}

The pipeline uses the open source program PSIPRED to predict the secondary structure state (coil, helix, sheet) at each amino acid position for both the wild type and the variant protein sequences. For each SNP the protein sequence was downloaded using the Uniprot API, where the SNP was swapped into the sequence. In total 21,094 wild type sequences and 74,393 SNP sequences were processed with PSIPRED on the HPC Wales cluster. For each SNP the probability change between each state of the position containing the SNP and the corresponding wildtype states were used as well as the overall predictions to produce 5 features for the final training data. The following bash script loads SNP co-ordinates from the humvar dataset into the uniprot API to retrieve protein sequences for each SNP:

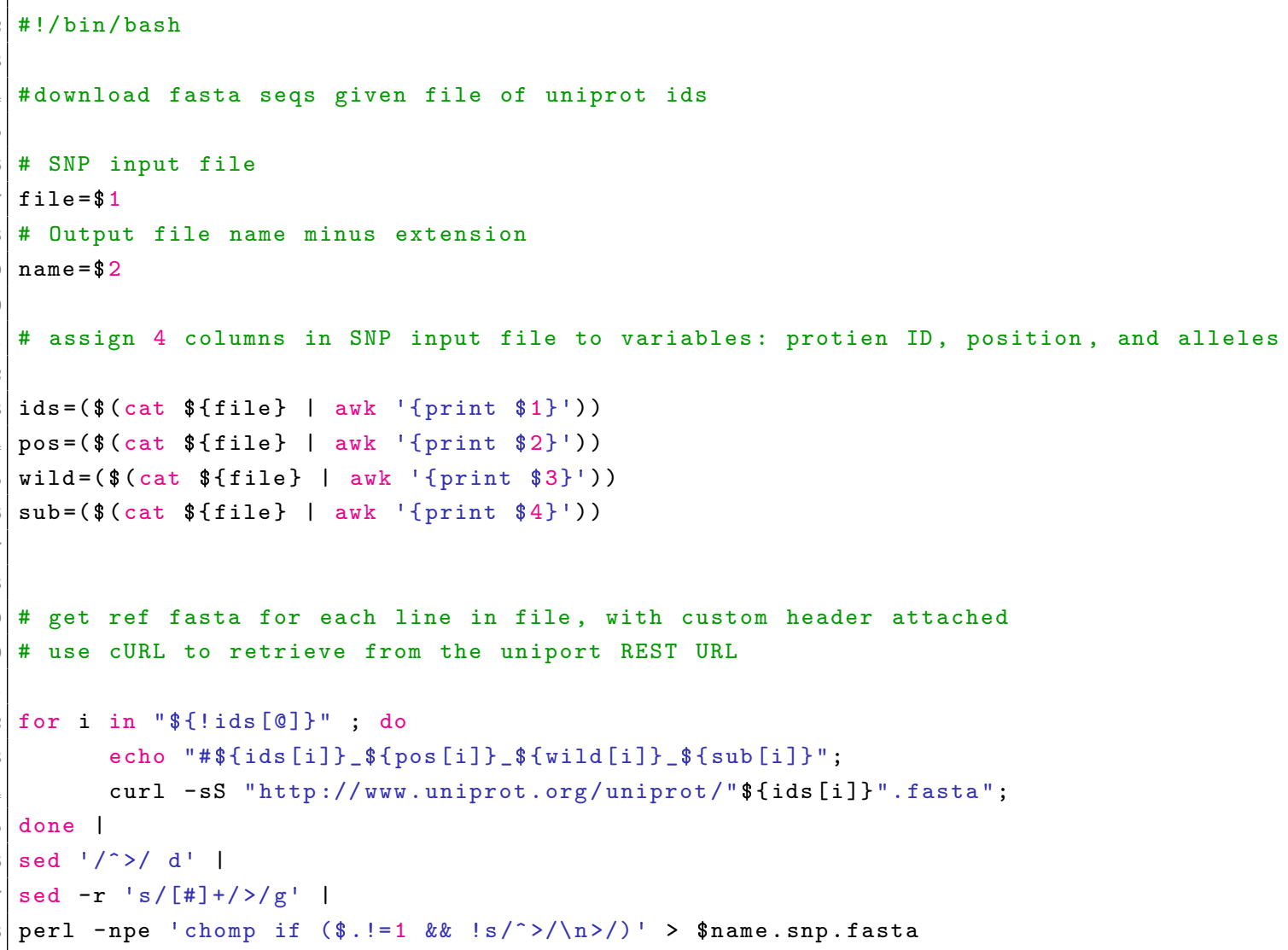

Figure 5.3: Bash script to retrieve fasta sequences for a file given in SNP format.

The awk script in figure 5.4 replaces the wild type amino acid at the SNP position with the mutated amino acid.

The sequences containing the wild type and the SNPs were then processed using 


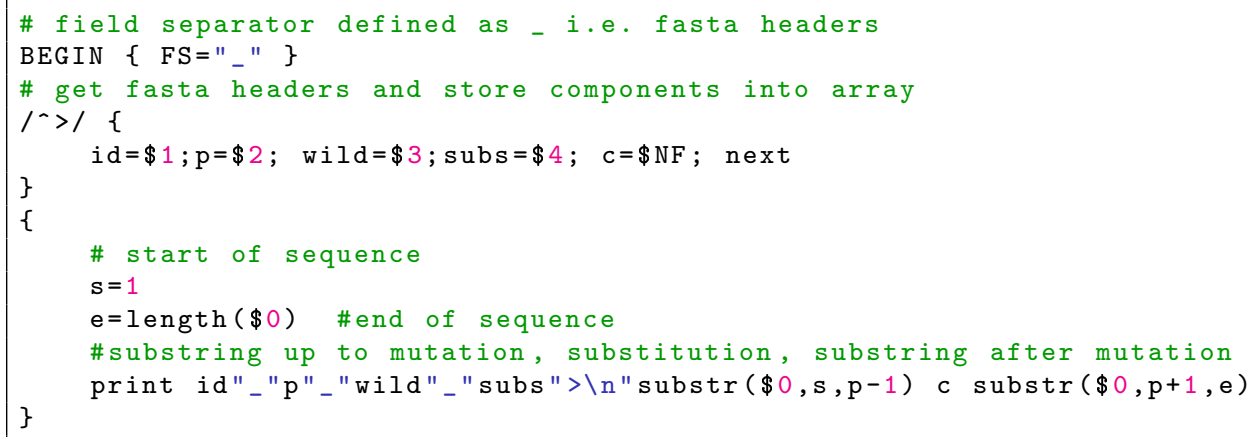

Figure 5.4: An AWK script that replaces the wild type amino acid with the mutation

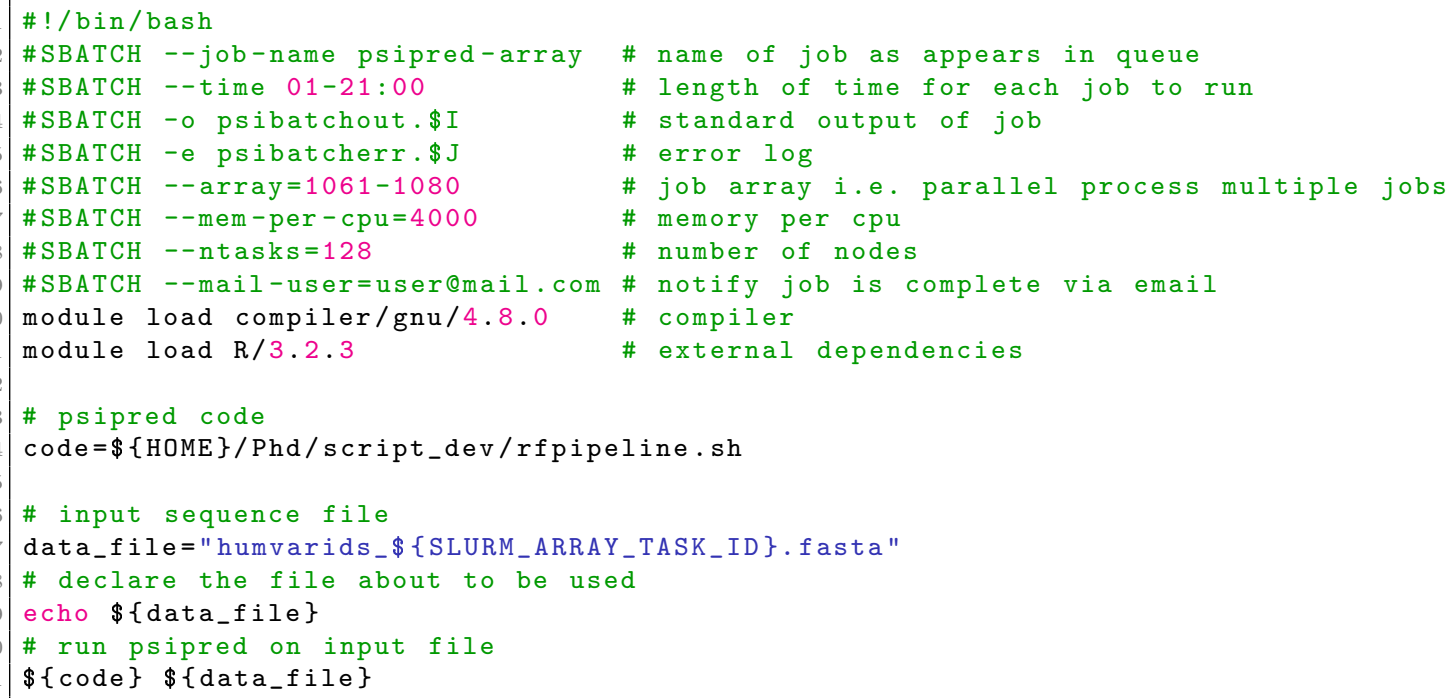

Figure 5.5: A SLURM job script run on the HPC Wales Portal that calls the psipred commandline facility. The script logs a job in a queue containing any other jobs users submit across HPC clusters, where parameters such as compiler, number of cores and how long the script should be allowed to run for. It takes $\mathbf{4 5}$ hours (time parameter 01-21:00) to process 300 sequences of varying length.

PSIPRED. Each sequence took on average 20 minutes to process on a standard desktop, therefore this task was completed by running PSIPRED on the HPC Wales cluster which reduced the time taken to 2-3 minutes. The SLURM script in 5.5 is the SLURM job schedule script that specifies a variety of parameters needed such as number of cores, run time and memory required to run psipred. The fair usage limit on the HPC Wales cluster allowed for a scheduled job to run up to 48 hours, which is the equivalent of processing 300 sequences, therefore the 21,094 wild type sequences and 74,393 SNP sequences were split into 318 jobs.

Each PSIPRED output file for a given SNP was compared to it's corresponding wild type protein using the bash script in figure 5.6. A sample comparison is given in Figure 5.7 which shows how the secondary structure prediction changes not only at 


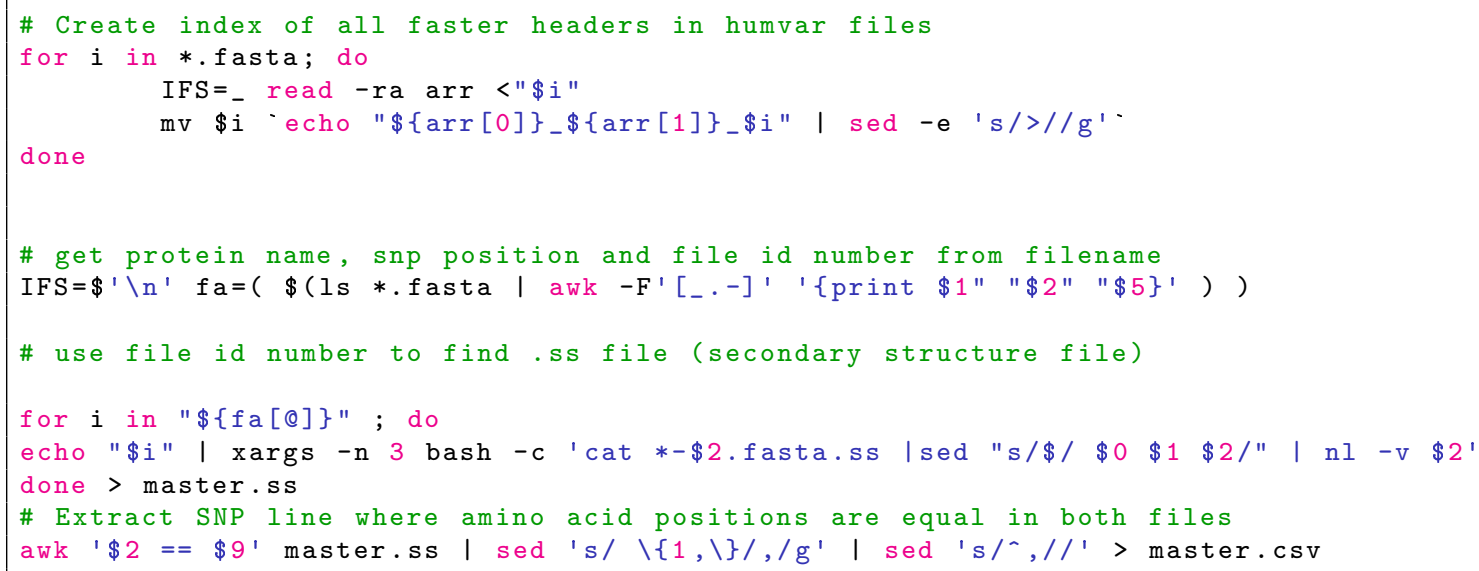

Figure 5.6: A bash script to process output of psipred.

the SNP position, but also in neighbouring SNPs. This is due to the underlying PSIBLAST alignment used when processing the wild type sequence and the mutated sequence, where a proportion of candidate protein sequences used in the secondary structure prediction in both cases will differ, however the largest changes are generally at the SNP position or next to it.

\section{$\begin{array}{lllllllllllllll}\text { M } & \text { C } & \text { D } & \text { A } & \text { K } & \text { V } & \text { M } & \text { R } & \text { K } & \text { C } & \text { Q } & \text { V } & \text { - Wild protein sequence }\end{array}$ $\begin{array}{llllllllllllll}\text { M } & \text { C } & \text { D } & \text { A } & \text { K } & \text { J } & \text { M } & \text { R } & \text { K } & \text { C } & \text { Q } & \text { V } & \text { - Sequence containing SNP }\end{array}$}

PSIPRED output wild

\begin{tabular}{llllrr}
\multicolumn{2}{l}{ Position Amino Acid Prediction } & C & H & S \\
1227 & M & H & 0.027 & 0.984 & 0.021 \\
1228 & C & H & 0.054 & 0.915 & 0.021 \\
1229 & D & H & 0.007 & 0.996 & 0.003 \\
1230 & A & $H$ & 0.007 & 0.999 & 0 \\
1231 & K & $H$ & 0.017 & 0.993 & 0.001 \\
1232 & V & H & 0.007 & 0.998 & 0.001 \\
1233 & M & C & 0.998 & 0.005 & 0.002 \\
1234 & R & C & 0.982 & 0.027 & 0.002 \\
1235 & K & C & 0.966 & 0.058 & 0.007 \\
1236 & C & C & 0.999 & 0 & 0.001 \\
1237 & Q & C & 0.915 & 0.054 & 0.021 \\
1238 & V & S & 0.040 & 0.100 & 0.860
\end{tabular}

\section{PSIPRED output SNP}

$\begin{array}{llllrr}\begin{array}{lll}\text { Position Amino Acid Prediction } \\ 1227\end{array} & \text { M } & \text { C } & \text { H } & \text { S } \\ 1228 & \text { C } & \text { H } & 0.017 & 0.984 & 0.031 \\ 1229 & \text { D } & \text { H } & 0.054 & 0.915 & 0.021 \\ 1230 & \text { A } & H & 0.007 & 0.996 & 0.003 \\ 1231 & \text { K } & \text { C } & 0.013 & 0.087 & 0 \\ 1232 & \text { J } & \text { C } & 0.064 & 0.195 & 0.015 \\ 1233 & \text { M } & \text { C } & 0.905 & 0.190 & 0.005 \\ 1234 & \text { R } & \text { C } & 0.023 & 0.002 \\ 1235 & \text { K } & \text { C } & 0.966 & 0.027 & 0.005 \\ 1236 & \text { C } & \text { C } & 0.097 & 0.001 & 0.002 \\ 1237 & \text { Q } & \text { C } & 0.915 & 0.054 & 0.021 \\ 1238 & \text { V } & \text { S } & 0.040 & 0.100 & 0.860\end{array}$

Figure 5.7: Comparison of two sample PSIPRED output files, where the left shows predictions for the wild type protein and the right shows the same sequence with the SNP is inserted. Lines are colour coded by increasing difference in prediction probabilities between the wild type and SNP sequence, where red depicts the largest difference and yellow the smallest. 


\subsubsection{Results}

After feature extraction the humvar dataset contained 14,266 pathogenic and 29,154 benign SNPs with all feature data. A 5-fold cross validation was performed in which for each fold $75 \%$ of the SNPs were randomly selected to form a training set, and the remaining $25 \%$ were used as an unseen test set on the trained classifiers. 6 classifiers were built using the training data: Random Forest, Decision Tree, Logistic Regression, Artificial Neural Network, Naive Bayes and Support Vector Machine (SVM). The R code in figure 5.8 shows the functions used to train and test each classifier.

Table 5.3 shows the results of each fold for each classifier and figure 5.9 shows a Receiver Operator Curve (ROC) plotting the sensitivity vs specificity of each classifier for it's mean scoring model in terms of accuracy across all scoring thresholds (normalized between 0 and 1 ) generated by the model. Figure 5.10 shows the importance of each feature when used in the Random Forest model to discriminate between pathogenic SNPs and neutral SNPs. The importance is measured by the difference in accuracy when excluding a feature and re-running the model compared to the model with all features used. 


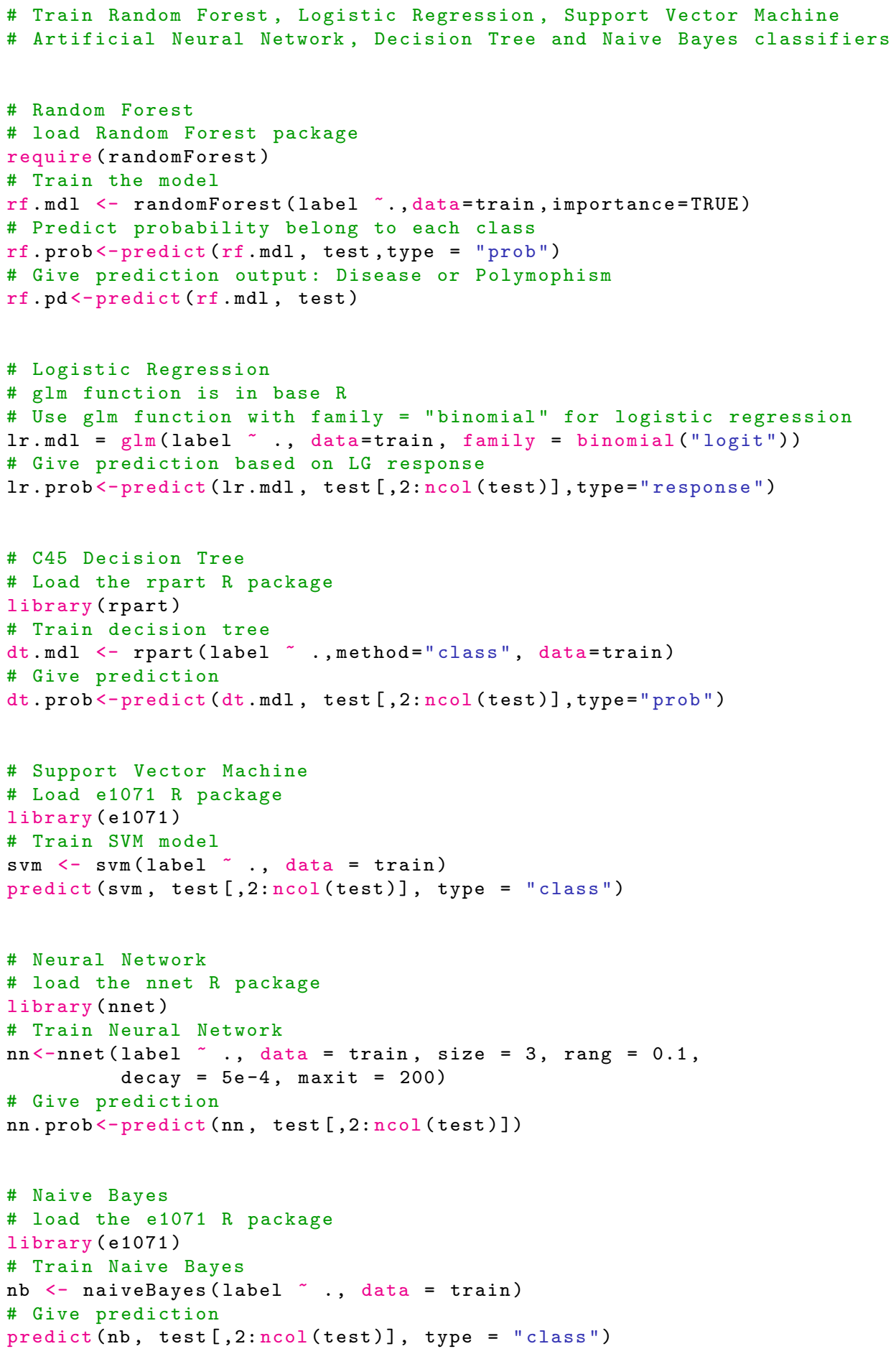

Figure 5.8: $R$ script used to train and test a Random Forest classifier 
Table 5.3: 5 fold cross-validation of the 6 classifiers.

\begin{tabular}{|c|c|c|c|c|c|c|c|c|c|c|}
\hline Algorithm & Fold & Disease & Neutral & $\mathbf{T P}$ & FP & FN & TN & TPR & FPR & Accuracy \\
\hline \multirow[t]{5}{*}{ Random Forest } & 1 & 4137 & 5426 & 3778 & 257 & 359 & 5169 & 93.63 & 93.51 & 93.56 \\
\hline & 2 & 4045 & 5518 & 3687 & 249 & 358 & 5269 & 93.67 & 93.64 & 93.65 \\
\hline & 3 & 4127 & 5436 & 3813 & 269 & 314 & 5167 & 93.41 & 94.27 & 93.9 \\
\hline & 4 & 4125 & 5438 & 3791 & 265 & 334 & 5173 & 93.47 & 93.93 & 93.74 \\
\hline & 5 & 4155 & 5408 & 3806 & 250 & 349 & 5158 & 93.84 & 93.66 & 93.74 \\
\hline \multirow[t]{5}{*}{ Logistic Regression } & 1 & 4211 & 5352 & 3692 & 343 & 519 & 5009 & 91.5 & 90.61 & 90.99 \\
\hline & 2 & 4109 & 5454 & 3593 & 343 & 516 & 5111 & 91.29 & 90.83 & 91.02 \\
\hline & 3 & 4195 & 5368 & 3725 & 357 & 470 & 5011 & 91.25 & 91.42 & 91.35 \\
\hline & 4 & 4154 & 5409 & 3680 & 376 & 474 & 5033 & 90.73 & 91.39 & 91.11 \\
\hline & 5 & 4153 & 5410 & 3685 & 371 & 468 & 5039 & 90.85 & 91.5 & 91.23 \\
\hline \multirow[t]{5}{*}{ Neural Network } & 1 & 4179 & 5384 & 3682 & 353 & 497 & 5031 & 91.25 & 91.01 & 91.11 \\
\hline & 2 & 4077 & 5486 & 3587 & 349 & 490 & 5137 & 91.13 & 91.29 & 91.23 \\
\hline & 3 & 4141 & 5422 & 3742 & 340 & 399 & 5082 & 91.67 & 92.72 & 92.27 \\
\hline & 4 & 4133 & 5430 & 3681 & 375 & 452 & 5055 & 90.75 & 91.79 & 91.35 \\
\hline & 5 & 4162 & 5401 & 3701 & 355 & 461 & 5046 & 91.25 & 91.63 & 91.47 \\
\hline \multirow[t]{5}{*}{ Naïve Bayes } & 1 & 4744 & 4819 & 3717 & 318 & 1027 & 4501 & 92.12 & 81.42 & 85.94 \\
\hline & 2 & 4652 & 4911 & 3614 & 322 & 1038 & 4589 & 91.82 & 81.55 & 85.78 \\
\hline & 3 & 4746 & 4817 & 3755 & 327 & 991 & 4490 & 91.99 & 81.92 & 86.22 \\
\hline & 4 & 4676 & 4887 & 3702 & 354 & 974 & 4533 & 91.27 & 82.31 & 86.11 \\
\hline & 5 & 4769 & 4794 & 3773 & 283 & 996 & 4511 & 93.02 & 81.91 & 86.63 \\
\hline \multirow[t]{5}{*}{ SVM } & 1 & 4241 & 5322 & 3775 & 260 & 466 & 5062 & 93.56 & 91.57 & 92.41 \\
\hline & 2 & 4128 & 5435 & 3640 & 296 & 488 & 5139 & 92.48 & 91.33 & 91.8 \\
\hline & 3 & 4220 & 5343 & 3768 & 314 & 452 & 5029 & 92.31 & 91.75 & 91.99 \\
\hline & 4 & 4190 & 5373 & 3742 & 314 & 448 & 5059 & 92.26 & 91.86 & 92.03 \\
\hline & 5 & 4239 & 5324 & 3769 & 287 & 470 & 5037 & 92.92 & 91.47 & 92.08 \\
\hline \multirow[t]{5}{*}{ C45 Decision Tree } & 1 & 4333 & 5230 & 3685 & 350 & 648 & 4880 & 91.33 & 88.28 & 89.56 \\
\hline & 2 & 4165 & 5398 & 3590 & 346 & 575 & 5052 & 91.21 & 89.78 & 90.37 \\
\hline & 3 & 4370 & 5193 & 3715 & 367 & 655 & 4826 & 91.01 & 88.05 & 89.31 \\
\hline & 4 & 4308 & 5255 & 3689 & 367 & 619 & 4888 & 90.95 & 88.76 & 89.69 \\
\hline & 5 & 4353 & 5210 & 3706 & 350 & 647 & 4860 & 91.37 & 88.25 & 89.57 \\
\hline
\end{tabular}




\section{Humvar Test Set Classifier Comparison}

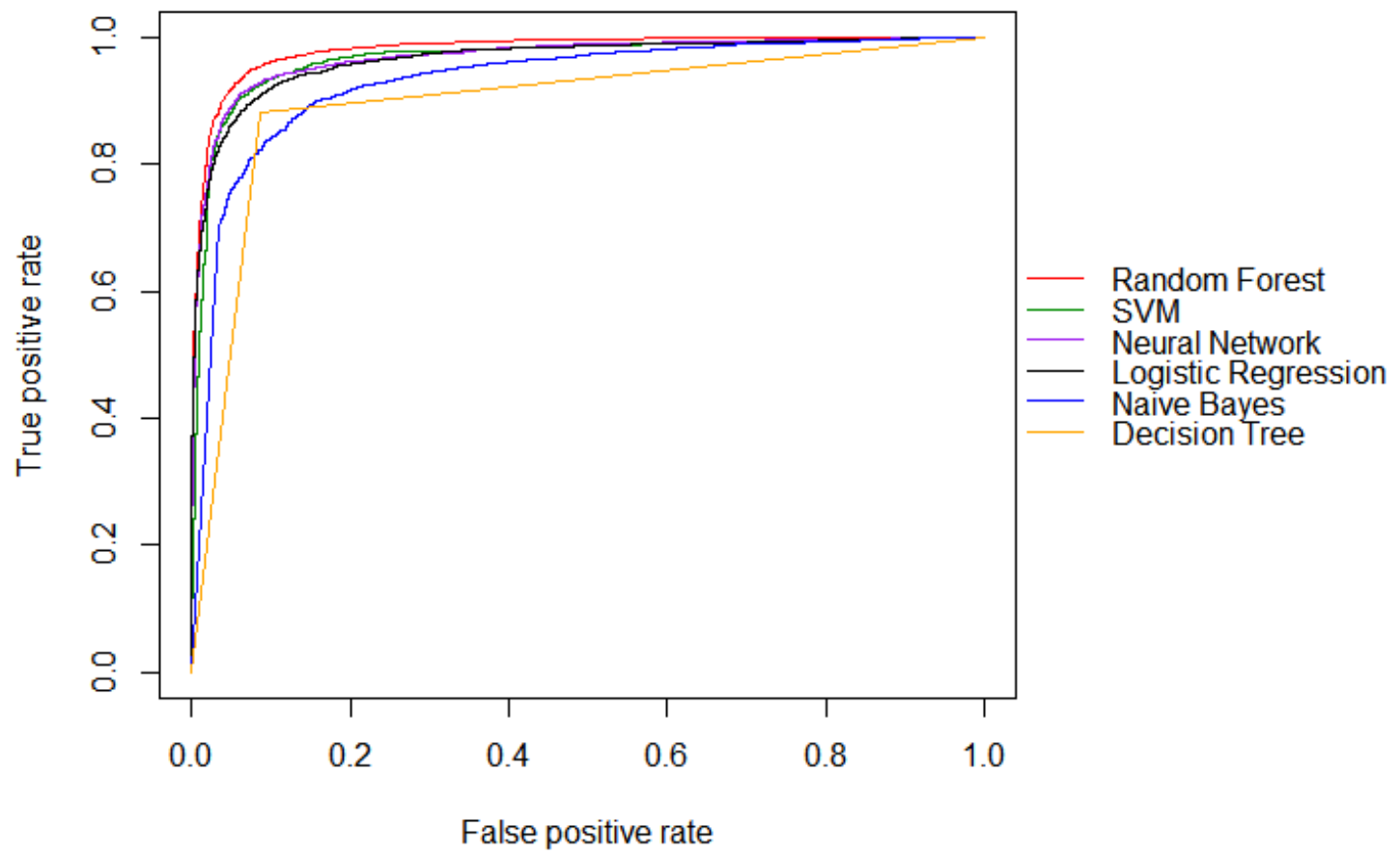

Figure 5.9: Feature importance ranked by the mean decrease in accuracy when each feature is excluded from the Random Forest model 


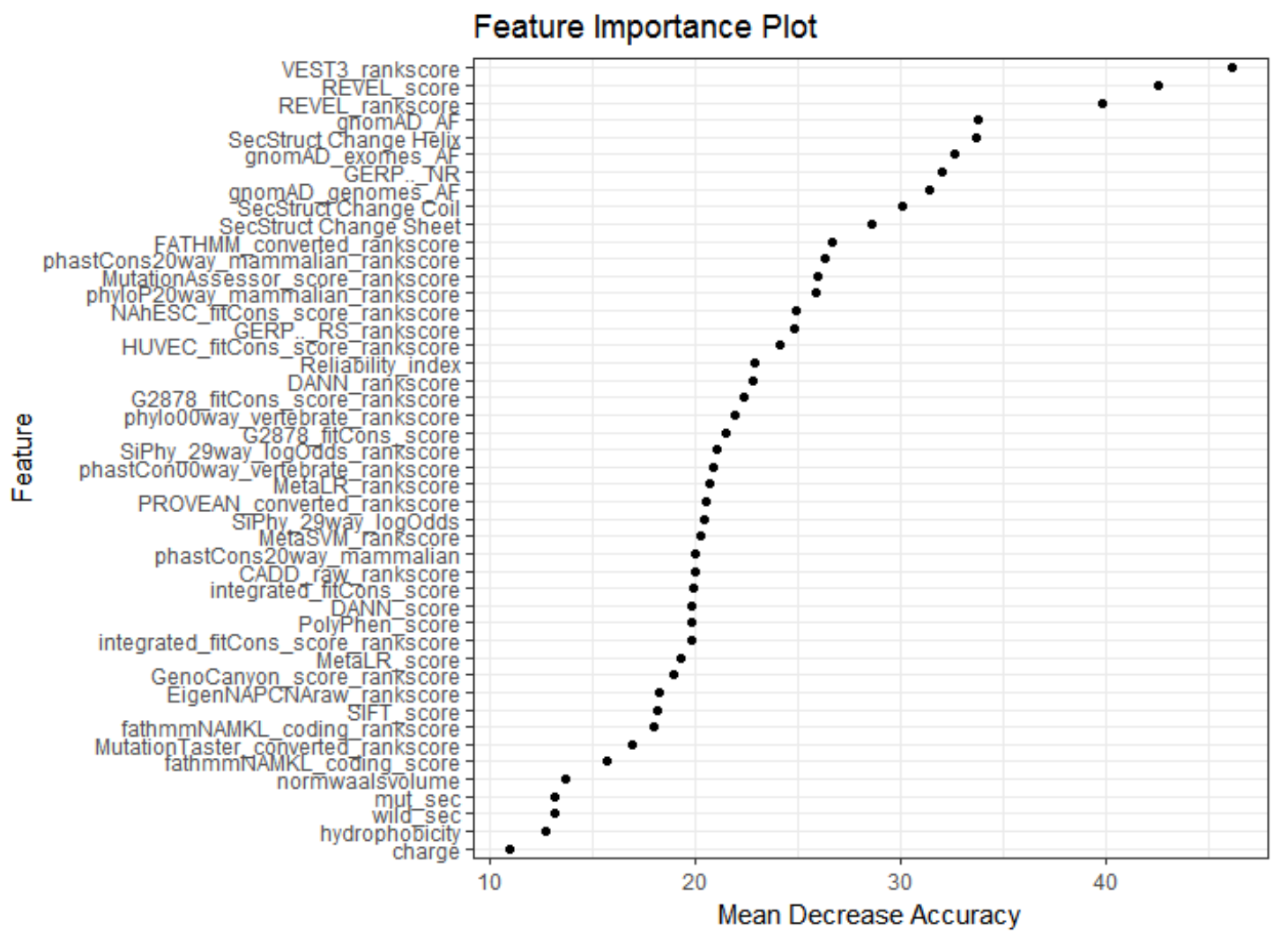

Figure 5.10: Feature importance ranked by the mean decrease in accuracy when each feature is excluded from the Random Forest model

\subsubsection{Comparison of Random Forest to other classifiers}

Random Forest was the most accurate classifier in every fold (93.56-93.9\% accuracy) and was chosen to be compared against existing classifiers. The model used in the 3rd best fold was used (fold 3 or 4 both had $93.74 \%$ accuracy) as this represented the mean accuracy in terms of all 5 Random Forest models. The results for the test set for each of these classifiers was collected as part of the feature extraction pipeline. The score for each classifier, for each SNP in the test set was compared to the class probability given by Random Forest and used to generate the ROC curve in Figure 5.11 . 


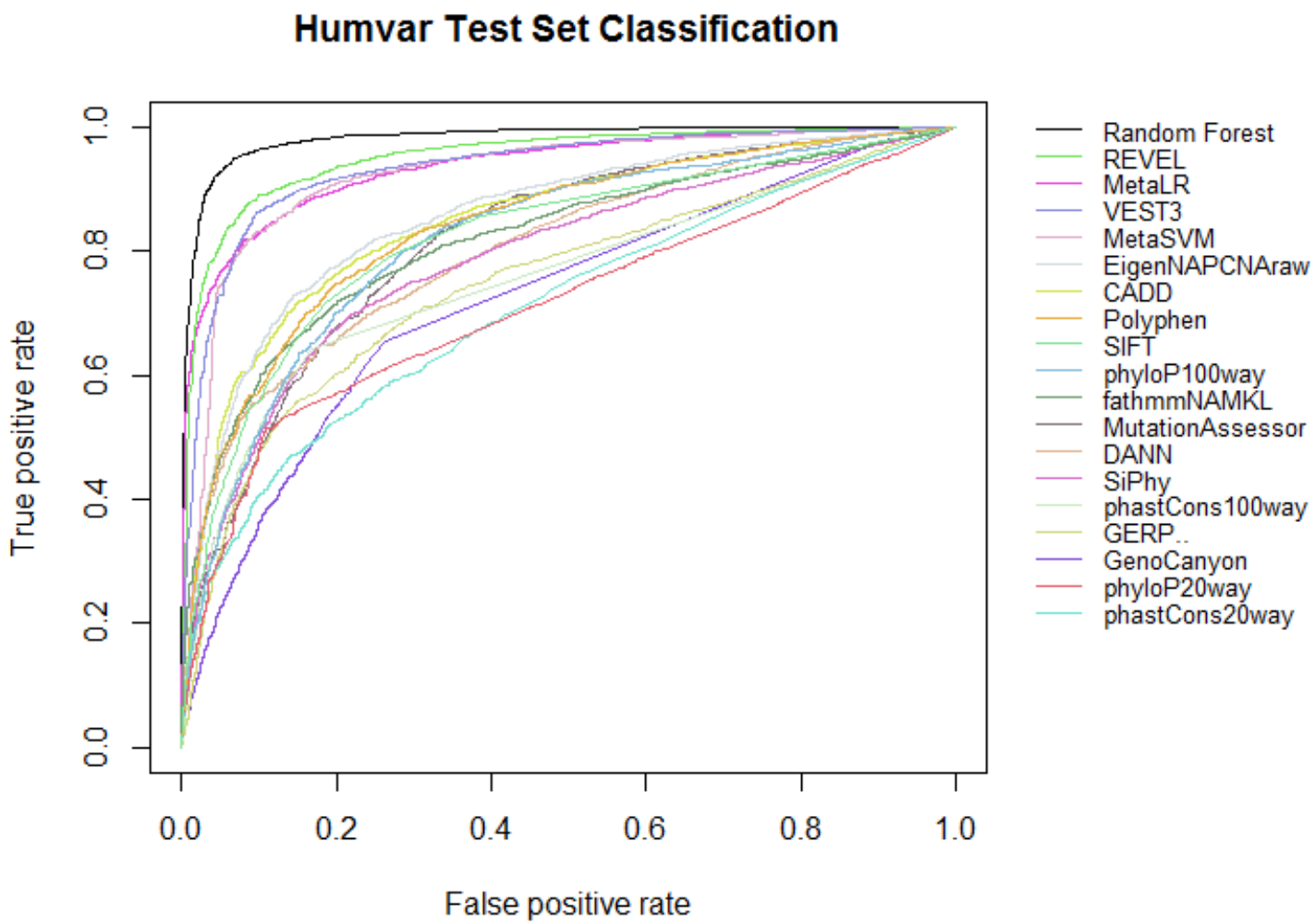

Figure 5.11: ROC curve comparing the classifier from this thesis (black) to scores from other classifiers when predicting disease/benign status on the humvar test set

Random Forest performed better than any of the other classifiers. Figure 5.12 shows the specificity of each algorithm when the sensitivity is set to $95 \%$ (for algorithms that could attain $95 \%$ sensitivity). 


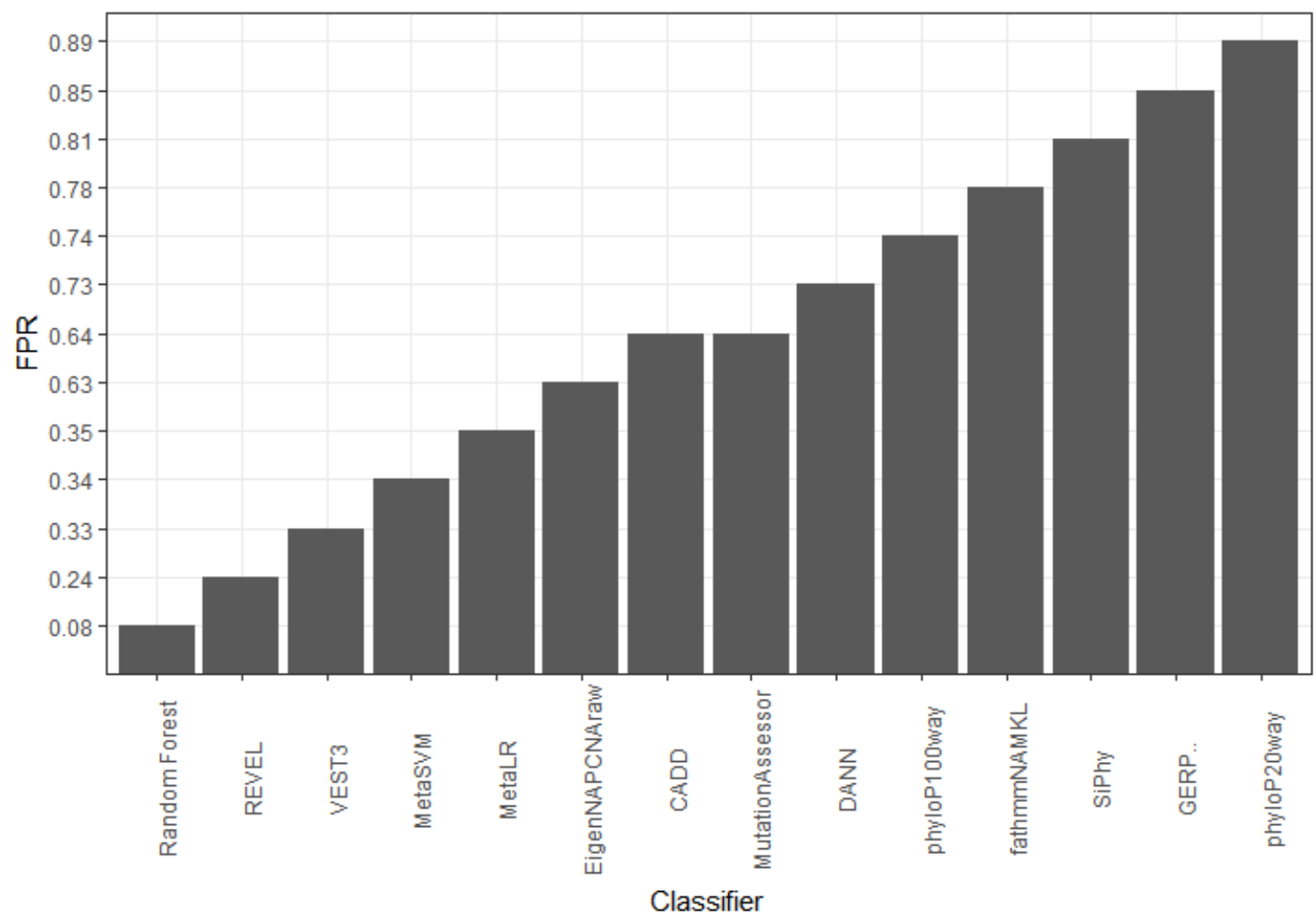

Figure 5.12: Specificity plot of each algorithm when sensitivity is set to $95 \%$. Only algorithms that could achieve $95 \%$ sensitivity are presented

\subsection{Functional Analysis of SNPs associated with Epilepsy}

The Clinvar dataset was queried for all SNPs found in genes that contain mutations known to cause Epilepsy. Using the Clinvar clinical significance guidelines https:// www.ncbi.nlm.nih.gov/clinvar/docs/clinsig/, query terms "Benign" were used to identify non-pathogenic SNPs, and "Pathogenic" were used to identify pathogenic SNPs. In total 251 pathogenic and 50 benign SNPs were identified. Table 5.4 shows which genes were selected when querying Clinvar. 
Table 5.4: Frequency table of genes associated with epilepsy sourced from Clinvar

\begin{tabular}{llllll}
\hline Gene & Count & Gene & Count & Gene & Count \\
\hline SCN1A & 153 & CPA6 & 3 & STX1B & 2 \\
KCNQ3 & 14 & EPM2A & 3 & CACNA1H & 1 \\
EFHC1 & 13 & PLPBP & 3 & CLN8 & 1 \\
LGI1 & 10 & POLG & 3 & DDHD2 & 1 \\
CHRNB2 & 9 & PRICKLE1 & 3 & GABRA1 & 1 \\
SCN9A & 9 & SCN1B & 3 & GABRB3 & 1 \\
ALDH7A1 & 8 & SPATA5 & 3 & GAL & 1 \\
RELN & 7 & CHRNA2 & 2 & GLDC & 1 \\
SLC2A1 & 6 & CNTNAP2 & 2 & KCNC1 & 1 \\
CHRNA4 & 5 & GABRG2 & 2 & MRI1 & 1 \\
MEF2C & 5 & KCNMA1 & 2 & NACC1 & 1 \\
SCARB2 & 5 & KCNQ2 & 2 & PRDM8 & 1 \\
ST3GAL5 & 5 & KCNT1 & 2 & & \\
NHLRC1 & 4 & SCN8A & 2 & & \\
\hline
\end{tabular}

Each SNP was used as input to the pipeline to collect they required features for classification using the Random Forest classifier trained using the humvar dataset. Each SNP was scored using Random Forest and figure 5.13 shows that Random Forest achieved higher accuracy (92\% accuracy, 93.2\% sensitivity, and 86\% specificity) when compared to other commonly used prediction scoring algorithms. Figure 5.14 shows the specificity of each classifier when set to $95 \%$ sensitivity (for classifiers that achieved at least $95 \%$ sensitivity), the confusion matrix in table 5.5 shows the overall predicted results and table 5.6 how many pathogenic SNPs were predicted for each gene. 


\section{Humvar Test Set Classification}

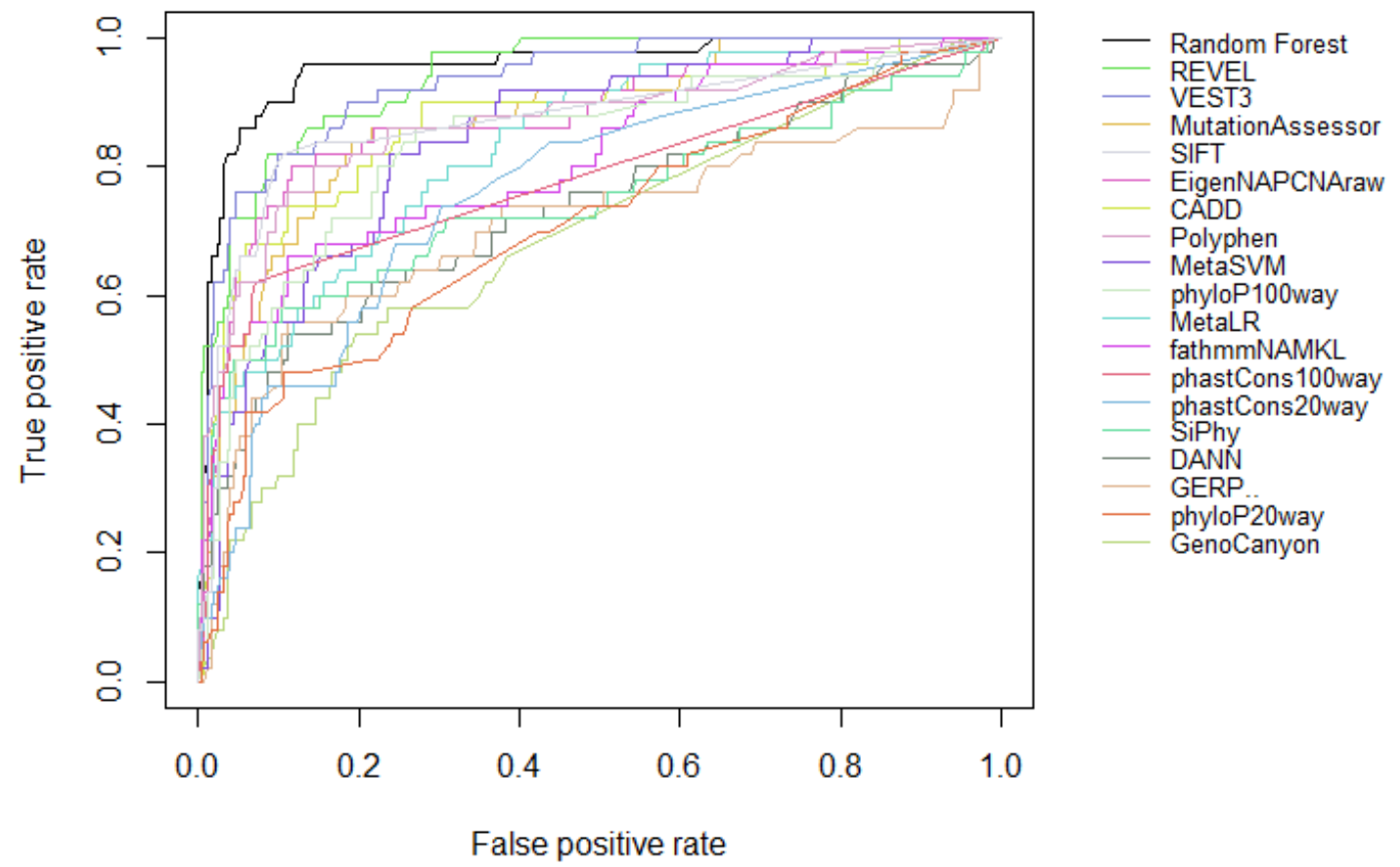

Figure 5.13: ROC curve comparing the classifier from this thesis (black) to rankscores from other classifiers when predicting disease/benign status for SNPs found in genes associated with epilepsy 


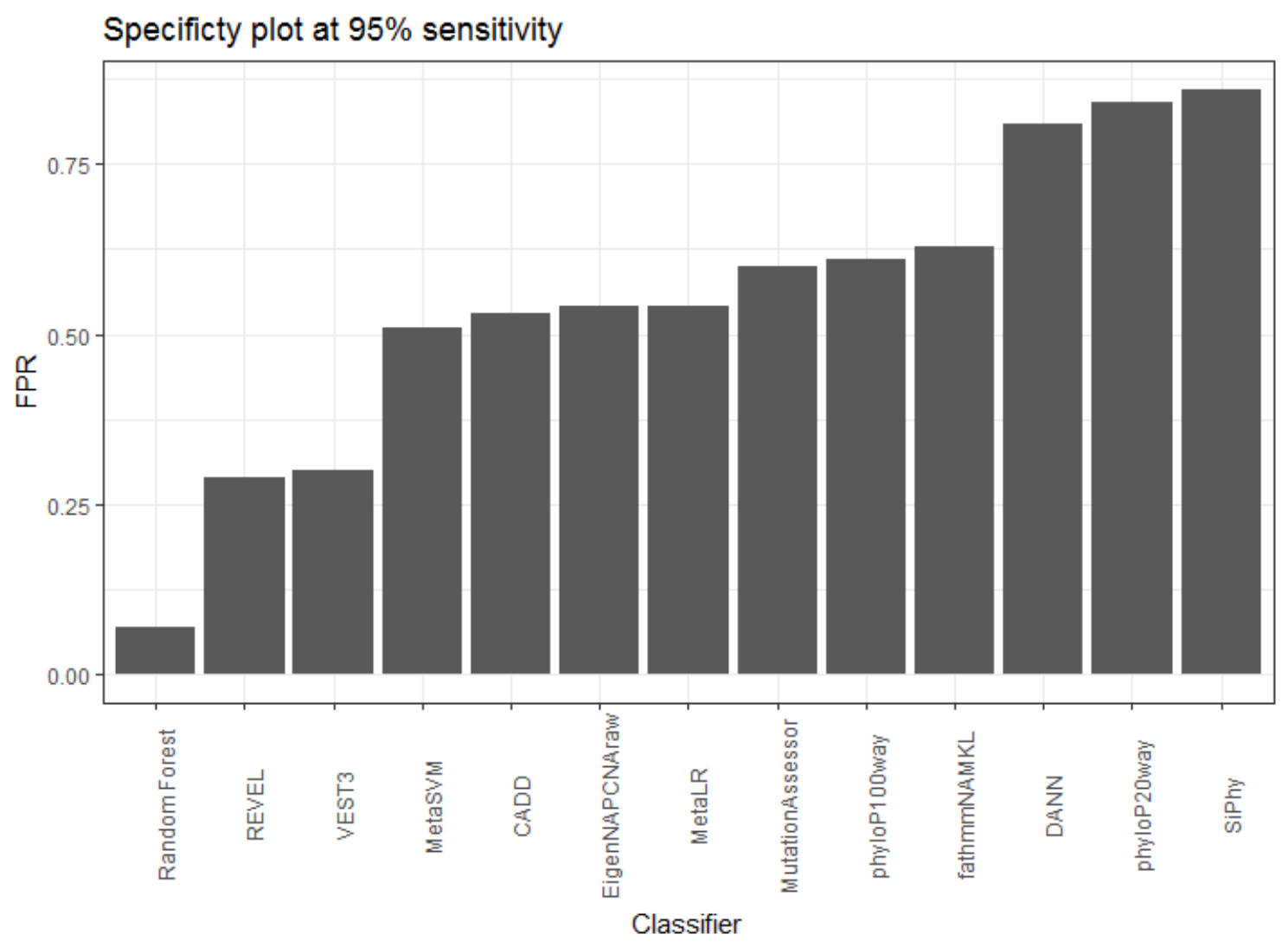

Figure 5.14: Specificity plot of each algorithm when sensitivity is set to $95 \%$. Only algorithms that could achieve $95 \%$ sensitivity are presented

Table 5.5: Confusion matrix for Random Forest classifier showing the number of observed vs predicted classifications in 301 SNPs found in genes associated with epilepsy.

\begin{tabular}{lll}
\hline & \multicolumn{2}{l}{ Predicted } \\
\hline Observed & Disease & Polymorphism \\
Disease & 236 & 15 \\
Polymorphism & 7 & 43 \\
\hline
\end{tabular}

\subsection{Summary of Results}

Predicting the effect of SNPs is an essential part of bioinformatics analysis that allows researchers to prioritize which SNPs should be analyzed using downstream processes in a laboratory setting. Many existing prediction scoring systems achieve high accuracy, but few offer both high sensitivity and specificity, as seen when comparing scoring systems on the humvar dataset. Scoring systems are mostly built from machine learning processes that use a variety of relevant protein features to train models, and as such some systems specialize in predicting the effect or certain SNPs, such as 
Table 5.6: Comparison of true pathogenic SNPs and predicted pathogenic SNPs in each gene

\begin{tabular}{llllll}
\hline Gene & $\begin{array}{l}\text { True } \\
\text { Pathogenic }\end{array}$ & $\begin{array}{l}\text { Predicted } \\
\text { Pathogenic }\end{array}$ & Gene & $\begin{array}{l}\text { True } \\
\text { Pathogenic }\end{array}$ & $\begin{array}{l}\text { Predicted } \\
\text { Pathogenic }\end{array}$ \\
\hline SCN1A & 150 & 149 & SCARB2 & 2 & 2 \\
LGI1 & 10 & 10 & SCN1B & 2 & 2 \\
ALDH7A1 & 8 & 7 & STX1B & 2 & 2 \\
CHRNB2 & 7 & 7 & CLN8 & 1 & 2 \\
RELN & 7 & 6 & DDHD2 & 1 & 1 \\
SLC2A1 & 6 & 6 & EFHC1 & 1 & 1 \\
MEF2C & 5 & 5 & EPM2A & 1 & 1 \\
CHRNA4 & 4 & 4 & GABRA1 & 1 & 1 \\
NHLRC1 & 4 & 3 & GABRB3 & 1 & 1 \\
SCN9A & 4 & 3 & GAL & 1 & 1 \\
CPA6 & 3 & 3 & GLDC & 1 & 1 \\
PLPBP & 3 & 3 & KCNC1 & 1 & 1 \\
POLG & 3 & 3 & KCNQ2 & 1 & 1 \\
PRICKLE1 & 3 & 2 & MRI1 & 1 & 1 \\
SPATA5 & 3 & 2 & NACC1 & 1 & 1 \\
ST3GAL5 & 3 & 2 & PRDM8 & 1 & 1 \\
GABRG2 & 2 & 2 & SCN8A & 1 & 0 \\
KCNMA1 & 2 & 2 & CACNA1H & 0 & 0 \\
KCNQ3 & 2 & 2 & CHRNA2 & 0 & 0 \\
KCNT1 & 2 & 2 & CNTNAP2 & 0 & 0 \\
\hline
\end{tabular}

ultra-rare SNPs or SNPs found in certain regions such as ion channels.

The approach taken in this chapter incorporates as much knowledge from existing scoring systems and commonly used features, as well as bespoke features derived with the use of various bioinformatics software. The aim is to train a classifier to achieve both high sensitivity and specificity in the capability to predict pathogenic SNPs. An automated feature extraction pipeline was built to allow ease of use when processing a large number of SNPs, as demonstrated during the classifier training process of this chapter. Multiple machine learning techniques were explored and compared to 14 other commonly- used prediction software, in which the Random Forest classifier trained in this study was able to achieve the highest accuracy amongst all prediction software.

A comparison of existing prediction software and the Random Forest classifier was also conducted for epilepsy specific SNPs, where an even larger increase in accuracy over the existing software was seen. Various other disease specific studies report reduced accuracy in SNP prediction using existing prediction software, which is hypothesized to be the use of non-disease specific training sets used to train classifiers. The results in this chapter show that epilepsy SNPs are also difficult to classify and show reduced accuracy when compared to the humvar dataset. However, the large increase in accuracy when compared to other software may be due to the inclusion of 
many different features from prediction software that may specialize in certain areas. This could also possibly due to the inclusion of structural features in the training set, where various SNPs that cause epilepsy occur in transmembrane proteins. In conclusion, the classifier trained in this chapter performs better than commonly used SNPs prediction software in a large non-disease specific SNP dataset, and performs even better than prediction software in epilepsy specific SNPs. 


\section{Chapter 6}

\section{Discussion}

\subsection{SAIL studies}

The case studies presented in the first results chapter demonstrate the ability to conduct powerful population level retrospective healthcare studies. Linkage of national datasets such as those held within the SAIL Databank can be used to facilitate novel studies in diseases such as epilepsy, where it is possible to measure the burden of epilepsy in terms of both health and social outcomes. The most important aspect of these results is the ability to define an accurate epilepsy cohort within the SAIL databank so that further downstream research can take place. The validation study to determine people with Epilepsy from GP records shows that data in SAIL can be compared to gold standard external datasets to achieve higher sensitivity, and arguably more importantly very high specificity to ensure only people with epilepsy are used for further studies, and a clear control group can also be defined.

While much research in epilepsy rightly focusses on seizure control, the SAIL databank can be used effectively to measure the social impact of living with epilepsy. It has long been thought that people living with epilepsy often suffer in terms of social deprivation because of factors such as employment and being able to hold a driving license. These results were able to measure the prevalence and incidence of epilepsy across all deciles of WIMD scores to which a strong trend of social deprivation and a diagnosis of epilepsy was found. Importantly, the results were able to address the question of social deprivation in terms of social drift or social causation by comparing social deprivation at time of diagnosis to a 10 year follow up period in which it could not be concluded that there is a strong trend in social drift following a diagnosis of Epilepsy. It is findings such as these that can be valuable to patients with a new diagnosis of epilepsy by giving some assurance that their diagnosis will not make 
much difference in terms of social status, while highlighting that extra support is needed given the existing deprivation profile of people with epilepsy.

It is also possible to link electronic healthcare records held within SAIL to administrative data sets such as the Department for Children, Education, Lifelong Learning and Skills in Wales to measure not just the social impact of someone who has epilepsy, but the educational outcomes of children born to mothers with epilepsy. The results in this chapter show that mothers being prescribed sodium valproate during pregnancy are observed to have children that have poorer attainment in national school tests when compared to a control cohort. This result emphasizes why seizure control is such a large research focus in epilepsy, but why it must also take into account situations not limited to effective seizure control. Other UK studies and audits have found that while sodium valproate prescribing is declining in women of child bearing age, it still remains high given the already known risks of reduced IQ and cognition of offspring exposed to sodium valproate inutero. These results also add the social impact of epilepsy in school results of children born to mothers with epilepsy, where it is clear these should be an increased focus on limiting sodium valproate prescriptions for women thinking of having children.

There are however clear limitations in all of these studies, which mainly entail important information not available form electronic health care records or linked data, rather than small sample sizes. For example it is not possible to determine the severity of epilepsy in the SAIL databank as it is often not recorded formal in GP records, and while it is possible to ascertain what antiepileptic drug has been prescribed to a patient, the exact daily dose is not recorded. The lack of some of these variables means it is not possible explore the effects of poor seizure control during pregnancy on cognitive outcomes of children, or to meausre the variance in social deprivation of people with epilepsy in terms of their seizure control. Even the definition of epilepsy found in GP records does accurately describe the type of epilepsy, such as focal or generalised epilepsy, and so it is not possible to study outcomes within sub-groups of people with epilepsy. To strengthen the impact of linked healthcare data in epilepsy research, these data gaps must be addressed in order to answer more nuanced research questions.

\subsubsection{Social deprivation and epilepsy}

The aim of this study was to investigate the relationship between social deprivation of people diagnosed with epilepsy, in particular if high levels of deprivation of chronic diseases such as epilepsy are due to social drift of social causation.

$8,100,232$ person years of healthcare records were used to calculated the prevalence 
and incidence of epilepsy in Wales and link them to the Welsh Index of Multiple Deprivation. The overall epilepsy prevalence using a combination of epilepsy diagnosis codes and AED prescriptions was $0.77 \%$ and incidence of 29.5/100 000 per year, which is comparable with other studies in developed countries [253], [51], [254], [49]. By comparing the prevalence of epilepsy in each WIMD decile, a strong association was shown between increased social deprivation as well as higher incidence of epilepsy. Both prevalence and incidence are doubled in the most deprived population decile compared to the least deprived decile (see table 3.5.

The increase in epilepsy incidence with increasingly deprived WIMD deciles initially suggested that the cause of higher epilepsy prevalence in more deprived deciles would be due to the movement from less deprived areas to more deprived areas following an epilepsy diagnosis. However, the follow up cohort study supports the hypothesis that the increased epilepsy prevalence in deprived areas is likely due to social causation rather than social drift (see table 3.7). While the higher prevalence of parents with epilepsy in deprived areas will inevitably produce more children with epilepsy, it is possible that acquired or symptomatic may play a larger role than epilepsy of a genetic origin in more deprived areas. It is difficult to obtain the cause of epilepsy in the SAIL Databank (either genetic or acquired), but given that more deprived areas have increased rates of risk factors for acquired epilepsy, such as perinatal hypoxic injury, head trauma, and cerebrovascular disease $[21,52,53]$ it is possible that living in more deprived areas leads to an increased risk of developing epilepsy.

Similar results have been reported in both the UK and internationally. A retrospective study in Wales found more patients with epilepsy living in deprived wards of residence as measured by the Townsend index [50], and the incidence of epilepsy in a prospective study across 20 GP practices in London and the South-East of England identified a strong association between epilepsy incidence and deprivation when comparing the Carstairs score between deprivation fifths, although the association was weaker inside London [51]. Another prospective study using adults in Iceland found that poorer socio-economic status is a risk factor for epilepsy [49].

Area level deprivation measures such as WIMD, Townsend and Carstairs score have limitations in both geographical granularity, and modelling all possible factors associated with social deprivation. As seen in the weaker deprivation effects found in London and the the weaker correlation in the geographical representation of major cities in Wales, area level deprivation is not entirely suitable to measure social deprivation, and individual level deprivation scores would benefit these types of studies. For example, it is possible in this study for two people living in the same area to have the same deprivation score, but in reality they would have different 
"individual deprivation," when considering more than geographic location, such as ease of access to services and employment. Similarly for people developing epilepsy in densely populated areas such as major cities, we might not expect to see much movement and therefore the effects of social drift may appear weaker.

These results add further evidence to support the argument that social causation, rather than social drift, could be responsible for an increase in higher social deprivation of people with epilepsy. This provides the opportunity to identify risk factors for epilepsy that could be targeted in areas of higher deprivation, as well as providing further evidence of the health impact of living in socially deprived areas.

Further work remains however. The WIMD score is based on an LSOA level, and it would be useful for future studies to study the effects of epilepsy on deprivation at an individual basis. For example, two people living in the same LSOA will clearly have different levels of social deprivation. There is potential however to link patient records to earnings, benefits and tax records using the Administrative Data Research Center https://adrn.ac.uk, which was set up to help researchers link healthcare records with administrative data such as those held in the Department of Work and Pensions. These data could potentially allow researchers to study how deprivation changes following a diagnosis of epilepsy in more detail.

While it was possible calculate a measure of sensitivity $90.5 \%$ for the algorithm used to determine epilepsy when comparing to patients in the Cardiff epilepsy register, it was not possible calculate the specificity due to lack of a control group. The prevalence of $0.77 \%$ provides an estimate of specificity, however a further work would validate this algorithm by using a control group of people that are known to definitely not have epilepsy. It would also be interesting to study the effects of epilepsy severity on social deprivation. Epilepsy severity is not well recorded in GP records, and so there needs to be a focus on enriching datasets routinely collected healthcare records with more detailed information on disease status.

\subsubsection{Validation of epilepsy algorithm using a gold standard dataset}

This study aimed to validate different algorithms for selecting people with epilepsy using anonymous GP patient records. The previous deprivation study used an algorithm that took into account epilepsy diagnosis codes and repeat AED prescriptions which cases identified as epilepsy by the algorithm were compared to the an epilepsy register with a gold standard diagnosis. This study used epilepsy patients and patients that definitely did not have a diagnosis of epilepsy sourced from 
Morriston hospital to perform a sensitivity/specificity analysis of three algorithms: Epilepsy diagnosis codes only, epilepsy diagnosis codes with a repeat AED prescription and an AED prescription only.

The results showed that by using both epilepsy diagnosis codes with a repeat AED, anonymised GP records can be used to accurately identify patients with epilepsy. This algorithm achieved sensitivity and specificity $84 \%, 87 \%, 79 \%$ and $99 \%, 98 \%$, $100 \%$ for all patients, adults and children respectively. These figures are comparable with sensitivities and specificities from other epilepsy validation studies in different healthcare systems e.g. Australian, Italian and American studies achieved sensitivities of $82-90 \%$ and specificities of $94-100 \%$ [236], [237], [238]. The results also showed that using a repeat AED prescription only may be the best approach when identifying children with epilepsy as it achieved $98 \%$ for both sensitivity and specificty. This algorithm would not be suitable for use on adults as it only achieved a specificity of $61 \%$. This can be explained by the widespread use of AEDs for indications other than epilepsy in adults (e.g. migraine, mood disorders and neuropathic pain). AEDs are seldom prescribed for indications other than epilepsy in children in the UK [239].

There was little difference in performance between using just an epilepsy diagnosis and both an epilepsy diagnosis and a repeat AED other than adding AEDs results in slightly higher specificity / lower sensitivity. GP diagnosis codes for epilepsy therefore seem reliable in their own right. Although this is expected, given that epilepsy diagnosis should be made in secondary care in the UK and later transcribed into the primary care record by GPs https://www.nice.org.uk/guidance/cg137, this has not been described in the literature and is an important result for future research involving GP epilepsy diagnosis codes. It would however be desirable to use an algorithm that maximizes specificity when selecting patients from anonymized GP records as it is a priority to be as certain as possible that someone identified with epilepsy does indeed have epilepsy, especially so if a cohort of epilepsy patients were to be compared against a control group for further study. Therefore using a repeat AED in addition to an epilepsy diagnosis is the preferred algorithm for identifying epilepsy in SAIL. Various reasons might include certain disorders such as non-epileptic attack disorder that present almost identical symptoms to that of an epileptic seizure which require detailed EEG examination to tell the difference. For EEGs to be accurate it requires a seizure to occur during an examination, and so it is difficult to conduct a thorough analysis, especially if seizures are infrequent, leading to some patients taking years to have a confirmation of non-epileptic attacks while being treated with AEDs.

The strengths of this study was that a gold standard dataset of patients with know 
epilepsy diagnosed by an epilepsy specialist was available as well as carefully selected patient records for patients that definitely did not have epilepsy. These data provided a robust validation of the three algorithms which could immediately be used to identify many epilepsy cases from the 2.8 million people with an anonymized GP patient record in SAIL. Although the reference population was of gold standard, it was a relatively small population due to the resources needed to manually check medical records and test results. Also, these results are specific to primary care records in Wales and are not applicable to other healthcare systems or methods of ascertaining epilepsy cases (for example hospital discharge summaries). Other parts of the UK do have similar healthcare systems and although the results may be generalizable

to the remainder of the UK further work needs to be done to prove this. Currently there is no facility to include EEG and imaging data within SAIL and so we could not include these in our ascertainment algorithms. Additionally it is impossible to identify people with epilepsy who do not attend their GP or have not been seen by a hospital specialist.

The reference epilepsy cohort was obtained from a secondary care epilepsy database which may have provided a bias towards people with more severe epilepsy, and thus more likely for an epilepsy diagnosis to be recorded in GP records. Also the group of people without epilepsy were sourced from patients who had attended general neurology clinics as a control group. This group therefore does not represent the 'general' population without epilepsy. However, this group of patients may be considered as a 'better test' of ascertainment algorithms as patients with other neurological conditions may be more likely to be incorrectly coded as having epilepsy than the general population. Conversely it is also possible (although unlikely in our opinion) that neurologists would not record a diagnosis of epilepsy in a general neurology clinic appointment with a different focus (e.g. headache).

\subsubsection{Educational attainment of children born to mother's with epilepsy}

This study aimed to compare the educational attainment of children born to mothers with epilepsy to a control group, with a particular focus on which AEDs mothers took during pregnancy. This study used Key Stage 1 results for mathematics, science and English/Welsh, a national school assessment for 2,196 children (440 with epilepsy, 1,756 control) at 7 years of age between 2003 and 2008 academic years.

The results showed an association between poorer school attainment and children exposed to valproate or AEDs in combination inutero. Compared with a matched control group, fewer children with mothers being prescribed sodium valproate during 
pregnancy achieved the national minimum standard in CSI $(-12.7 \%$ less than the control group), mathematics $(-12.1 \%)$, language $(-10.4 \%)$ and in science $(-12.2 \%)$. Even fewer children with mothers being prescribed multiple AEDs during pregnancy achieved a national minimum standard: CSI (by $-20.7 \%$ less than the control group), mathematics $(-21.9 \%)$, language $(-19.3 \%)$ and science $(-19.4 \%)$

The results support previous studies that provide consistent evidence that inutero exposure to sodium valproate and AEDs in combination are linked to adverse neurodevelopmental outcomes. In contrast there was no difference seen in children exposed to carbamazepine, lamotrigine or mothers that did not take drugs during pregnancy, however it is impossible to accurately test for significance in the lamotrigine group as the sample size is small. While mothers not prescribed any AED during pregnancy do not appear to give birth to children that have decreased educational attainment, it is possible that this group of women have less frequent seizures, thus reducing the risks to the unborn child associated with exposure to seizures inutero.

Other studies have also studied the effect of intuero exposure to sodium valproate and the effect on children's IQ. The NEAD study found a 9-point decrease in IQ in children at 3 and 6 years old who were born to mothers taking sodium valproate during pregnancy $[27,243]$ as well as decreased motor, emotional and behavioural/adaptive functioning in children at 3 years old [255]. Studies based on the UK Epilepsy and Pregnancy Register have associated sodium valproate with a decrease in cognitive development and early cognitive delay that suggests children are at a disadvantage well before school age $[32,256]$. While this study finds a statistically non-significant trend in language at KS1, other studies have shown decreased language and verbal skills at early infant stage [257, 258]. Some of the studies mentioned have found that increased AED dosage plays a part in cognitive impairment, however due to a lack of dosage information in the SAIL databank this could not be explored. While some of the mentioned studies associate exposure to carbamazepine with some forms of cognitive impairment, there are also studies that suggest carbamazepine has no effect on intelligence; these results supports the latter with no evidence of decreased educational attainment at school age [259].

The strength of this study is the ability to select a large cohort of 440 children with national test results without major recruitment bias and compare to a large control group. Using a standardized national assessment as a measure of performance ensures that each child has the opportunity to be assessed based on the same curriculum, and as such these results would closer reflect the learning experience of children at this age compared to an IQ test. The main limitation of these results are not being 
able to use maternal intelligence quotient (IQ) as a covariate as in [27, 243], which are not recorded in the SAIL databank.

The SAIL Databank also lacks information on various other potential covariates such as epilepsy severity and seizure frequency during pregnancy which may effect cognitive function of unborn child, or if the mother was taking folic acid during pregnancy as this is available "over the counter". The AED data was based on prescriptions, and so it is impossible to comment on adherence, however there is no reason to suspect that adherence differs greatly between different AEDs. It is also possible that mothers with poorly controlled seizures may have an effect on their child's education in terms of parental support outside of school settings, but this information is difficult to ascertain and is not available to any comprehensive standard within the SAIL databank. Another limitation of this study is that we are unable to report on AED dosage, although other studies have reported significant cognitive impairment even at low dosages of sodium valproate.

While these results highlights the risk of cognitive effects in the children of mothers prescribed sodium valproate or multiple AEDs, it is important to acknowledge that some epilepsies are difficult to treat without these treatment regimes. Despite this, these results add to the growing evidence that inutero exposure to certain AEDs can cause developmental problems in children, to which sodium valproate has recently been banned for use in women of child bearing age unless a pregnancy prevention

programme is in place https://www.gov.uk/guidance/valproate-use-by-womenand-girls.

\subsection{Natural Language Processing of epilepsy clinic letters}

This study aimed to validate an NLP algorithm developed to extract epilepsy specific information from unstructured clinic letters. A rules-based system was built using an open source NLP framework to extract details of epilepsy diagnosis, seizure type, seizure frequency, status of EEG, MRI and CT investigations. The main purpose of the NLP algorithm is to enrich routinely collected data sets such as those demonstrated in the previous chapter using the SAIL databank.

The algorithm was able to extract epilepsy information from a corpus of 200 clinic letters, written by 6 different clinicians, with an overall precision, recall and F1 score of $91 \%, 81 \%$ and $85 \%$ on a per item basis. As expected, the algorithm performed best in extracting clinic date and date of birth (F1 scores of $98 \%$ and 
$99 \%$ ) given that these fields consist of fixed format dates which are relatively easily to extract. In terms of epilepsy-specific information the algorithm performed best for medication $(\mathrm{F} 1=95 \%)$, confirming a diagnosis of epilepsy (93\%), epilepsy type $(84 \%)$ and presence of focal seizures $(81 \%)$. These items are frequently mentioned and presented in a relatively standard format e.g. medication is usually stated as drug name-strength-unit-frequency, and diagnosis appears at the top of letters in structured lists with or in text with clear references to the patient such as "she has focal epilepsy" or "her current medication is" within the main text.

For example, a letter may confirm temporal lobe epilepsy three times but only one mention of temporal lobe epilepsy is required to correctly classify that person's epilepsy. In this context extracting only one mention of temporal lobe epilepsy is just as useful as extracting all three. In the "per letter" test we, therefore, aggregated multiple mentions within a category in each letter to a binary decision based on the algorithm's ability to extract at least one true positive mention. In the above example if the algorithm had only correctly identified one of the three mentions of temporal lobe epilepsy we would have scored it as having a recall of $100 \%$ on a per letter basis but only $33 \%(1 / 3)$ on a per item basis. For the medication annotation, in the per letter approach, only a full list of the drugs prescribed with the respective doses was considered to be a positive outcome.

The algorithm was less accurate in identifying CT (57\%), MRI (75\%) and EEG results $(78 \%)$, seizure frequency $(66 \%)$ and generalised seizure terms $(66 \%)$. The two main reasons for not picking up such terms were due to mapping issues to UMLS, or the highly varied ways these terms are reported in clinic letters. For example, UMLS contains terms such as, "EEG with irregular generalized spike and wave complexes", however, it is often the case that when reported in text there are variety of words between the EEG and the finding e.g. "EEG was found to show generalized spike and wave complexes" or "There was no evidence of generalised spike-wave complexes when reviewing her EEG", and so this problem was approached by creating custom gazetteers that map to smaller terms such as "spike and wave" or "EEG", and writing JAPE rules to associate the finding with the EEG. Similarly the reporting of seizure frequency is highly varied e.g. "she had 5 seizures since March last year" or "1-2 focal seizures every evening". Seizure frequency is also often reported with terms such as "events" and "episodes" rather than defined seizure types, hence additional JAPE rules were built to accommodate such terms as part of seizure frequency in the presence of an epilepsy diagnosis.

Although every item in a letter was compared to that of a clinician, it is practical to provide a binary decision for some categories. If epilepsy is confirmed 3 times in a 
letter, the important information is that epilepsy is confirmed. For this reason the "per letter" score was developed where if 1 true positive for a epilepsy confirmed, epilepsy type, seizure type or abnormal/normal investigation, then the "per letter" score was given a true positive finding by the algorithm. The basis for this decision boundary is based on the high precision of the "per item" score, where there is high confidence a positive identified by the algorithm is a true positive. The "per letter" score achieved higher scores for precision, recall, and F1-score $(96 \%, 87 \%, 91 \%)$ on a per letter basis. The "per letter" approach for categories containing multiple mentions could be used with higher confidence than on an individual mention basis, as well as providing a practical way to summarise information from clinic letters. Additionally a "per person" measure (results summarised over several letters) could be used to determine epilepsy status as there will normally be several letters per person over a period of time.

Other studies demonstrate that NLP is being increasingly used for clinical information extraction purposes [260]. Performance of specific phenotype extraction algorithms developed as part of the i2b2 project using cTAKES (Apache clinical Text Analysis and Knowledge Extraction System) and HITex (Health Information Text Extraction) showed that for an NLP approach high PPV (precision) and sensitivity (recall) was achieved for extracting the following phenotypes; Crohn's disease $(98 \%, 64 \%)$, Ulcerative Colitis $(97 \%, 68 \%)$, MS (94\%,68\%), and Rheumatoid arthritis $(89 \%, 56 \%)$ [139]. As we aimed to extract epilepsy specific information other than a confirmed diagnosis, a recent study on patients with known MS identified from electronic healthcare records used NLP techniques to extract attributes specific to MS with high PPV and sensitivity, namely EDSS (Expanded Disability Status Scale) (97\%,89\%), T25FW (Timed 25 Foot Walk) $(93 \%, 87 \%)$, MS subtype $(92 \%, 74 \%)$ and age of onset $(77 \%, 64 \%)$ [140]. This study took into account items attributable only to the patient, as opposed to family members, which is an important distinction and interesting area of study in terms of identify potential risk factors for disease development. A study used clinic letters available at www.mtsamples.com to determine whether sentences containing disease and procedure information were attributable to a family member using the BioMedICUS NLP system, which achieved and overall precision, recall and F1-score of $91 \%, 94 \%$ and $92 \%$ [141].

There are however only a few published studies of clinical epilepsy information extraction systems. Cui et al developed the rule based Epilepsy Data Extraction and Annotation (EpiDEA) system which extracts epilepsy information from epilepsy monitoring unit discharge summaries. EpiDEA achieved an overall prevision, recall and F1 score of 94\%, 84\% and 89\% when extracting EEG pattern, past medications and current medication from 104 discharge summaries from Cleveland, USA [143]. 
Cui et al also developed the rule-based Phenotype Exaction in Epilepsy (PEEP) pipeline [144]. PEEP extracted epileptogenic zone, seizure semiology, lateralising sign, interictal and ictal EEG pattern with an overall precision, recall and F1 score of $93 \%, 93 \%$ and $92 \%$ in a validation set of 262 epilepsy monitoring unit discharge summaries from Cleveland, USA. Sullivan et al used a machine based learning NLP pipeline to identify a rare epilepsy syndrome from discharge summaries and EEG reports in Phoenix USA and achieved a precision, recall and F1 score of $77 \%, 67 \%$ and $71 \%$ respectively.

The main strength of this study was the use of a gold standard dataset of de-identified clinic letters to accurately extract novel data types from free texts that are not well populated in electronic healthcare records. The algorithm was built using open source technology so that the algorithm is easily shareable and can be run on potentially millions of letters as NLP tasks can be parallelism. The algorithm was able to make use of two open source plugins that have been used for information extraction tasks previously, as well as widely used medical ontologies that produce easily interpretable annotations that can be adopted for healthcare research [261] [244]. The algorithm was developed to extract epilepsy specific information, however this aim was met by filtering out non-disease specific information. It is possible that the algorithm could be adapted for other diseases with relative ease and many rules were built to capture language rules in general, not just medical item tagging. These rules could be adapted for more nuanced tasks such as finding frequency of events such as depressive episodes or migraines. Another advantage was that all rules are programmed in a relatively simple scripting language (JAPE) where other NLP systems rely heavily on the ability to program in more complicated languages such as Java. For this reason it is possible that clinicians themselves are more likely to participate in writing their own rules and embed medical expertise more readily into the algorithm or algorithms developed in future, for example adding in custom dictionaries for colloquial terms or coding a particular phrase that is meaningful when reported in clinic letters.

The main limitation of this study was the sample size of letters used to develop and test the algorithm. This is a limitation across many NLP tasks that focus on information extraction as it is labour intensive to manually annotate letters in the detail required to develop information extraction systems. Most information extraction systems typically use hundreds of letters rather than thousands. However even though this study only used 200 letters to test the accuracy of the algorithm, 1925 individual items were compared to those of an epilepsy specialist. Another limitation was the use of one specialist to annotate the letters used for comparison, where it is possible that annotations are affected by reviewer fatigue, and it was not possible to produce an inter-annotation agreement score if multiple clinicians annotated the letters. The 
address this a review of all letters that contained a disagreement between the human annotations and the algorithm annotations (174 letters out of the 200 letters) was conducted. The review showed that indeed the human annotator errors tended to be missing true positive items, which at first lead to a higher number of false positives produced by the algorithm where the human annotator corrected their annotations. In future multiple specialists are recommended for information extraction tasks.

While existing medical ontologies were used to tag items in the clinic letters, and rules were built to arrange which particular order of tagged terms describes a concept, medical ontologies such as UMLS, SNOMED-CT, READ and ICD were not built to aid information extraction from clinical free texts. Many descriptions of codes in these ontologies are too structured to reflect the language used in clinic letters and thus reduces recall in information extraction tasks. It is however important to produce annotations that adhere to existing medical ontologies, and so future work to address this would include developing methods to add some flexibility when matching terms in clinic letters to ontology descriptions. Much work has been done in so called "fuzzy matching" where sequences of words called "n-grams" are mapped to each other using word-vectorization and similarity measures [125] [262] [263]. Also it would be interesting to incorporate machine learning classification tasks where detailed information extraction is not needed. For example it might be possible to use machine learning to classify EEG reports as either normal or abnormal, without worrying about extracting every item that may indicate abnormalities.

\subsection{Predicting pathogenicity of SNPs for large datasets}

This study aimed to understand the different approaches for predicting pathogenicity of SNPs and to build a pipeline that sources a variety of SNP annotation and scoring data that is used to build an accurate classifier for SNPs. Many existing software have significantly better sensitivity that specificity, or vice-a-versa, and some specialize in capturing rare pathogenic variants or variants found within certain protein domains such as transmembrane regions. Most classifiers are non-disease specific, but some studies have shown that the accuracy reported on non-disease specific test sets are not matched in some disease only test sets. As well as building a non-disease specific SNP classifier, the classifier was also built to accurately classify epilepsy SNPs.

The results in this study showed that by using a mixture of common SNP annotations such as conservation scores, existing software scoring systems and bespoke features such as secondary structure prediction, the Random Forest classifier was able to 
score higher than all of the highest performing software currently available on both a non-disease specific dataset as well as showing a further increase in accuracy when using an epilepsy dataset. The classifier also achieved both high sensitivity and specificity, which some classifiers achieved high sensitivity or specificity. Constructing a ROC curve and using the class probability of predictions allowed the Random Forest classifier to be compared at different sensitivity thresholds to compare the corresponding specificity across all other classifiers, in which at a sensitivity threshold of $95 \%$, the Random Forest classifier achieved $97 \%$ specificity which was higher than the closest classifier, REVEL (92\% specificity). Six different classifiers were compared during the development phase using a 5 -fold cross validation coupled with feature selection techniques to try and improve the accuracy of each algorithm. Internal parameters of the classifiers were also tuned to achieve higher accuracy, although this did not have much of an effect. It was interesting that aside from using the Naive Bayes classifier, many of the algorithms showed higher accuracy than any of the other existing classifiers used for comparison. This suggests that the samples in the training set and the features used in the training process many have play more of a role in classification accuracy than a difference in choosing different classifiers.

Prediction accuracy of new software as presented in their original publications state high accuracy in non-specific disease datasets such as Humvar, but often vary in accuracy when used for a particular disease. Given that researching a specific disease is a common use case of research groups, it is important to know that the accuracy stated for an algorithm is not expected to be reproduced in other datasets. A study that compared the prediction accuracy of 17 different classifiers on SNPs in limb-girdle muscular distrophy (LGMS) showed that the Polyhen Humvar classifier achieved just $70.2 \%$ accuracy where in the Polyphen paper an accuracy of $86 \%$ is stated, with a similar trend for the majority of the other classifiers used in the comparison [264]. A study that selected 23 genes associated with immunity compared PolyPhen2, SIFT, MutationAssessor, Panther, CADD, and Condel classifiers in which only $20 \%$ of pathogenic SNPs were predicted as such, and over $45 \%$ of neutral SNPs were classified as pathogenic [265]. A larger non-disease specific study using 40,000 variants from the Phencode and dbSNP databases found that accuracy ranged from 15-65\% across MutPred, nsSNPA-nalyzer, Panther, PhD-SNP, PolyPhen, PolyPhen2, SIFT,SNAP, and SNPsGO [266].

There have been various studies which have explored creating disease specific classifiers that have reported higher accuracy than non-disease specific classifiers. A cancer specific machine learning based classifier was developed using common SNP annotation for 6326 missense SNPs that are known to be drivers for various subtypes of cancer, achieving 93\% accuracy [267]. Machine learning classifiers that dominate SNP 
prediction require large datasets with many features, in which there are not enough pathogenic SNPs in the majority of individual disease areas to train a classifier by using only damaging SNPs in the disease of interest. This is certainly true for epilepsy, and as such the approach taken was to include many features other than traditional conservation scores. Some of the prediction scores used in the classifier include features that specify which protein domain a SNP is found in such as transmembrane or ion channel regions as well as incorporating the predicted secondary structure of the SNP location as well as modelling the difference in predicted secondary structures between sequences containing the wild type and the SNP. Many pathogenic SNPs in genes associated with epilepsy such as SCN1A are located in ion channels, where any structural change in these channels can effect cellular excitability and induce seizures [268]. It is possible that some features used in the RF classifier are able to contribute towards improving classification accuracy in epilepsy related SNPs.

\subsubsection{Use of existing predictors as features}

Including the prediction and scores of existing software as a feature for machine learning purposes is not a novel idea. Earlier attempts to improve classification accuracy when relatively few prediction software existed involved combining these scores and weighting them into a single score using a statistical approach. The Combined Annotation scoRing toOL (CAROL) normalizes polyphen and SIFT scores by their standard normal deviations to achieve a $1 \%$ increase on that of their respective scores using a test set of 1,959 pathogenic and 9,691 neutral SNPs [269].

\subsubsection{Use of physiochemical properties and predicted secondary structure}

Most SNP prediction programs that use secondary structure as features obtain this information from known protein databases and uses the secondary structure status where the SNP is located as reported in the wild type protein. Querying secondary structure in this manner produces many missing data as only a proportion of proteins have been studied and had their secondary structure profile reported. This study used secondary structure prediction to obtain a predication of the secondary structure for every SNP, and modelled the difference in prediction between wild type and SNP sequences. These differences were reported as the percentage change across three secondary structure domains, in which they were ranked as the 5th, 9th and 10th most important features (out of 47 features) used in the Random Forest model.

It is difficult to define " difference in secondary structure" between a wild type sequence, and a sequence containing a SNP. The secondary structure of both sequences are 
predicted, not measured in a laboratory. The only thing that can be measured using PSIPRED is the likelihood of a single amino acid falling into the three categories of a beta sheet, alpha coil or helix. While this may be useful, particularly in some strict complexes of a protein, it is actually not important to this pipeline to determine what the effect of a SNP may have on a predicted secondary structure category. Firstly this is because it is not feasible to determine such a change for a SNP via prediction only, but mainly that the underlying methodology to secondary structure prediction is similar to sequence homology and evolutionary analysis of proteins - methods that already play a prominent role in functional prediction programs. Secondary structural prediction programs are extensions of multiple sequence alignments, where known secondary structures are incorporated later as a ground truth to assess structure based on these alignments. It is this inference between multiple sequence alignments and the disparity in structural prediction between a wild type sequence and a SNP sequence that is important.

\subsection{Future Work}

The SAIL case studies in this thesis showed that epilepsy patients can accurately be determined from GP records. The algorithm developed could be used to create an epilepsy register within the SAIL databank to facilitate further epilepsy research and be used in multiple studies. These studies have shown that socio-economic and national education datasets can be linked to epilepsy patients. One future project would be to link the educational outcomes of children who have epilepsy compared to a control group or other neurological conditions. It could be possible to study sub groups of children by taking into account what prescriptions they were taking during the school year.

There are limitations, however to the SAIL databank. Rich information found in clinic letters were largely absent from rountinely collected healthcare records. The next step would be to use the validated NLP application developed in chapter 4 to link rich epilepsy data from clinic records to the SAIL databank. This would require producing a version of the NLP application that could run on distributed systems. The lack of clinic letters available for NLP studies due to patient identifiers present is a major limitation that is not reflected with the SAIL databank. Forming a governance model to analyse identifiable patient data would be an important future project to help facilitate NLP research. Another barrier is not only the lack of clinic letters, but the lack of letter annotation by a clinician. Every NLP algorithm needs to be scored against a human annotator, and generating annotated training sets requires a lot of time. With crowd sourcing becoming popular it may be possible 
to open up annotation tasks to a wider participation group through crowd sourcing platforms.

Some categories of information from the NLP chapter were difficult to obtain accurately. Seizure frequency is an important piece of information that provides a level of severity of epilepsy, and would be an important covariate in an epilepsy study. The language describing seizures frequency and investigation reports remain to varied to capture all of the nuances with a rule based approach. Machine learning is one way to add flexibility into capturing these categories, and one immediate improvement would be to create methods of matching short phrases and slang terms to UMLS concept descriptions so that NLP applications aren't relying on exact phrase matching. Much work has been done on word embeddings to infer semantic similarity of words, however some future work in this area could expand this to phrases.

SNP prediction tools are numerous and show effectiveness in different disease areas. The pipeline developed in chapter 6 successfully incorporated existing SNP prediction tools as features alongside bespoke protein features to produce higher accuracy than all common SNP scoring tools in a disease non-specific dataset as well as epilepsy specific SNPs. With advances in technology powering big data, accurate tools will become an increasingly important tool in prioritising which SNPs warrant further research/ Future work would be to set up the pipeline developed as a web service to be used freely for research. This could help laboratories focus on building assays for a smaller group of SNPs. The pipeline could also be used to prioritise SNPs that may be linked to the SAIL databank. The ability to identify patients with certain conditions and co-morbidities and link them to SNPs may allow researchers to discover associations between SNPs and disease. Future work will include linking exomes and genomes from Welsh patients collected as part of the Wales Epilepsy Research Network to the SAIL databank. These patients have well defined epilepsy genotypes and it would be interesting to explore any potential trends in co-morbidities within subgroups of epilepsy syndromes such as exploring if different SNPs for the same epilepsy syndrome have a a higher propensity form experiencing co-morbidities such as migraines.

\subsection{Conclusions}

Epilepsy is a common disease that can have an impact on health and social well being. Epilepsy can also affect family members such as children born to parents with epilepsy. Advances in big data have provided the opportunity to explore the impact

of epilepsy on at population levels to uncover trends in healthcare data for people with epilepsy where insufficient data previously existed. 
The SAIL Databank not only provides national healthcare datasets, but it also socio-economic and administrative datasets that can all be linked anonymously. Data linkage is a powerful tool that allows datasets to be aligned to produce novel research, and the ability to do so anonymously speeds up research in terms of using a governance model that is exempt from ethical approval. A study using 8.1 million person-years of data was used to identify a strong trend between increased social deprivation and the incidence and prevalence of epilepsy. The study found that increased deprivation was likely to due to social causation rather than social drift. This finding was contrary to other studies that suggest chronic conditions such as epilepsy cause social drift after diagnosis.

A study of GP coding habits showed that it is possible to use GP records as a data source for identifying patients with epilepsy to be used for epidemiology studies. An algorithm was developed that found a combination of repeat AED prescriptions and a diagnosis of epilepsy was the most effective way to identify epilepsy from GP records, which gave $88 \%$ sensitivity and $98 \%$ specificity. This algorithm was used to select mother's with epilepsy who had children with Key Stage 1 education data to compare attainment between a large control group. This study found that for children exposed to sodium valproate or a combination of AEDs inutero there was a $12.7 \%$ and $19.8 \%$ decrease in attainment when compared to the control group.

Each SAIL study showed that there were various limitations in the data i.e. not having epilepsy type or AED daily dose. The Natural Language Processing study showed that there is potential to source rich patient information that is typically missing from routinely collected data. A validation of 200 epilepsy clinic letters showed that a rule-based NLP application can accurately identify patients with epilepsy (88.5\%), epilepsy type (84.5\%) and prescriptions (95\%). Other categories such as seizure frequency and investigation outcomes were more difficult to capture, however high specificity is reported across all categories. Aggregating all mentioned per category per letter achieved even higher accuracy and would be a practical approach to analysing large volumes of letters.

A SNP prediction pipeline was developed using the Random Forest machine learning classifier to determine the pathogenicity of SNPs. Validation on a large disease non-specific SNP dataset showed that the Random Forest classifier produced more accurate results than all other commonly used SNP prediction software, and was able to achieve $95 \%$ sensitivity with a specificity of $92 \%$. The classifier also achieved the highest accuracy on a dataset of 301 SNPs reported in epilepsy genes, and confirms findings in other studies than disease specific SNP datasets can pose a more difficult challenge in terms of predicting SNP pathogenicity. 


\section{Appendices}

\section{Appendix item 1: Read codes used for epilepsy definition}

Table 6.1: Read codes used to signify a diagnosis of epilepsy.

\begin{tabular}{|c|c|c|c|}
\hline Code & Description & Code & Description \\
\hline F25B. & Alcohol-induced epilepsy & $\mathrm{F} 25 \mathrm{y} 2$ & Localisation related epilepsy \\
\hline F25y 4 & Benign Rolandic epilepsy & F25D. & Menstrual epilepsy \\
\hline $\mathrm{F} 2545$ & Complex partial epileptic seizure & F2511 & Neonatal myoclonic epilepsy \\
\hline F25y3 & Complex partial status epilepticus & 667B. & Nocturnal epilepsy \\
\hline $\mathrm{F} 25 \mathrm{y} 0$ & Cursive (running) epilepsy & F25y. & Other forms of epilepsy \\
\hline F25C. & Drug-induced epilepsy & F25yz & Other forms of epilepsy NOS \\
\hline F259. & $\begin{array}{l}\text { Early infant epileptic encephalopathy } \\
\text { wth suppression bursts }\end{array}$ & F251y & $\begin{array}{l}\text { Other specified generalised convulsive } \\
\text { epilepsy }\end{array}$ \\
\hline F25.. & Epilepsy & F250y & $\begin{array}{lll}\text { Other } & \text { specified } & \text { generalised } \\
\text { nonconvulsive epilepsy } & \end{array}$ \\
\hline 1030. & Epilepsy confirmed & F 25 y 5 & Panayiotopoulos syndrome \\
\hline F25z. & Epilepsy NOS & F 254. & $\begin{array}{l}\text { Partial epilepsy with impairment of } \\
\text { consciousness }\end{array}$ \\
\hline F2544 & Epileptic automatism & $\mathrm{F} 254 \mathrm{z}$ & $\begin{array}{l}\text { Partial epilepsy with impairment of } \\
\text { consciousness NOS }\end{array}$ \\
\hline F2503 & Epileptic seizures - akinetic & F255. & $\begin{array}{l}\text { Partial epilepsy without impairment of } \\
\text { consciousness }\end{array}$ \\
\hline F2502 & Epileptic seizures - atonic & F255z & $\begin{array}{l}\text { Partial epilepsy without impairment of } \\
\text { consciousness NOS }\end{array}$ \\
\hline F2512 & Epileptic seizures - clonic & F255y & $\begin{array}{l}\text { Partial epilepsy without impairment of } \\
\text { consciousness OS }\end{array}$ \\
\hline F2513 & Epileptic seizures - myoclonic & $\mathrm{F} 2500$ & Petit mal (minor) epilepsy \\
\hline F2514 & Epileptic seizures - tonic & F252. & Petit mal status \\
\hline F25y1 & Gelastic epilepsy & F25F. & Photosensitive epilepsy \\
\hline F251. & Generalised convulsive epilepsy & F258. & Post-ictal state \\
\hline F251z & Generalised convulsive epilepsy NOS & F2541 & Psychomotor epilepsy \\
\hline F250. & Generalised nonconvulsive epilepsy & F2542 & Psychosensory epilepsy \\
\hline F250z & Generalised nonconvulsive epilepsy NOS & F2501 & Pykno-epilepsy \\
\hline F2510 & Grand mal (major) epilepsy & F2561 & Salaam attacks \\
\hline F2516 & Grand mal seizure & F2551 & Sensory induced epilepsy \\
\hline F253. & Grand mal status & F2556 & Simple partial epileptic seizure \\
\hline F2560 & Hypsarrhythmia & F2552 & Somatosensory epilepsy \\
\hline $\mathrm{F} 256 \mathrm{z}$ & Infantile spasms NOS & F25E. & Stress-induced epilepsy \\
\hline F2504 & Juvenile absence epilepsy & F2515 & Tonic-clonic epilepsy \\
\hline F25A. & Juvenile myoclonic epilepsy & SC200 & Traumatic epilepsy \\
\hline F257. & Kojevnikov's epilepsy & F2555 & Unilateral epilepsy \\
\hline F2505 & Lennox-Gastaut syndrome & F2553 & Visceral reflex epilepsy \\
\hline F2543 & Limbic system epilepsy & F2554 & Visual reflex epilepsy \\
\hline
\end{tabular}

\begin{tabular}{llll}
\hline Code & Description & Code & Description \\
\hline dn3e.* & ARBIL MR 200mg m/r tablets & dnp7. & LYRICA 300mg capsules \\
dn3f. & *ARBIL MR 400mg m/r tablets & dnp2. & LYRICA 50mg capsules \\
dn2.. & *BECLAMIDE & dnp3. & LYRICA 75mg capsules \\
dn2z. & *BECLAMIDE 500mg tablets & dn6.. & METHYLPHENOBARBITAL \\
dnc1. & *CLOBAZAM SLS 10mg capsules & dn6z. & METHYLPHENOBARBITONE 200mg \\
& & & tablets \\
\hline
\end{tabular}


Table 6.2 AED Read codes - Continued ...

\begin{tabular}{|c|c|c|c|}
\hline Code & Description & Code & Description \\
\hline do1z. & *DIAZEPAM 20mg/4mL injection & dn6x. & $\begin{array}{l}\text { METHYLPHENOBARBITONE } 30 \mathrm{mg} \\
\text { tablets }\end{array}$ \\
\hline do1B. & *DIAZEPAM 20mg/5mL RecTubes & dn6y. & $\begin{array}{l}\text { METHYLPHENOBARBITONE } 60 \mathrm{mg} \\
\text { tablets }\end{array}$ \\
\hline $\operatorname{dn} 53$. & *EMESIDE 250mg capsules & dna1. & MYSOLINE 250mg tablets \\
\hline $\operatorname{dn} 3 \mathrm{~A}$. & *EPIMAZ 100mg tablets & dna2. & $\begin{array}{l}\text { MYSOLINE } \quad 250 \mathrm{mg} / 5 \mathrm{~mL} \quad \text { oral } \\
\text { suspension }\end{array}$ \\
\hline $\operatorname{dn} 3 B$. & *EPIMAZ 200mg tablets & dna3. & MYSOLINE 50mg tablets \\
\hline $\operatorname{dn} 3 \mathrm{C}$. & *EPIMAZ 400mg tablets & dnj4. & NEURONTIN 100mg capsules \\
\hline $\operatorname{dn} 51$. & *ETHOSUXIMIDE 250mg capsules & dnj5. & NEURONTIN 300mg capsules \\
\hline $\operatorname{dn} 52$. & *ETHOSUXIMIDE $250 \mathrm{mg} / 5 \mathrm{~mL}$ elixir & dnj9. & $\begin{array}{l}\text { NEURONTIN } 300 \mathrm{mg} \text { capsules/600mg } \\
\text { tablets titration pack }\end{array}$ \\
\hline dn5y. & *ETHOSUXIMIDE $250 \mathrm{mg} / 5 \mathrm{~mL}$ elixir & dnj6. & NEURONTIN 400mg capsules \\
\hline $\operatorname{dn} 79$. & ${ }^{*}$ GARDENAL $200 \mathrm{mg} / 1 \mathrm{~mL}$ injection & dnj7. & NEURONTIN 600mg tablets \\
\hline dn7a. & *LUMINAL 15mg tablets & $\operatorname{dnj} 8$. & NEURONTIN 800mg tablets \\
\hline dn7b. & *LUMINAL 30mg tablets & dng2. & NOOTROPIL $1.2 \mathrm{~g}$ tablets \\
\hline $\operatorname{dn} 7 \mathrm{c}$. & ${ }^{*}$ LUMINAL $60 \mathrm{mg}$ tablets & dng3. & NOOTROPIL 33 \\
\hline $\operatorname{dn} 21$. & *NYDRANE 500mg tablets & dng1. & NOOTROPIL $800 \mathrm{mg}$ tablets \\
\hline dnba. & *ORLEPT 200mg e/c tablets & dnbA. & ORLEPT $200 \mathrm{mg} / 5 \mathrm{~mL}$ sugar free liquid \\
\hline dnbb. & *ORLEPT 500mg e/c tablets & dnb9. & $\begin{array}{l}\text { ORLEPT STARTER PACK } 200 \mathrm{mg} \text { e/c } \\
\text { tablets x10 }\end{array}$ \\
\hline do52. & *PARALDEHYDE injection 10mL & dnm.. & OXCARBAZEPINE \\
\hline do51. & *PARALDEHYDE injection 5mL & dnmx. & OXCARBAZEPINE 150mg tablets \\
\hline $\operatorname{dn} 98$. & *PENTRAN 100mg tablets & dnmy. & OXCARBAZEPINE 300mg tablets \\
\hline $\operatorname{dn} 97$. & *PENTRAN 50mg tablets & dnmz. & OXCARBAZEPINE 600mg tablets \\
\hline $\operatorname{dn} 63$. & *PROMINAL 200mg tablets & dnmw. & $\begin{array}{l}\text { OXCARBAZEPINE } 60 \mathrm{mg} / \mathrm{mL} \text { sugar } \\
\text { free oral suspension }\end{array}$ \\
\hline $\operatorname{dn} 61$. & *PROMINAL 30mg tablets & do5.. & PARALDEHYDE \\
\hline $\operatorname{dn} 62$. & *PROMINAL 60mg tablets & $\operatorname{dn} 7 .$. & PHENOBARBITAL \\
\hline do13. & *STESOLID 20mg/4mL injection & $\operatorname{dn} 74$. & PHENOBARBITAL 100mg tablets \\
\hline $\operatorname{dn} 3 \mathrm{H}$. & *TERIL CR 200mg m/r tablets & $\operatorname{dn} 71$. & PHENOBARBITAL 15mg tablets \\
\hline $\operatorname{dn} 3 \mathrm{I}$. & *TERIL CR 400mg m/r tablets & $\operatorname{dn} 7 \mathrm{~d}$ & PHENOBARBITAL 15mg/5mL elixir \\
\hline $\operatorname{dn} 55$. & *ZARONTIN 250mg capsules & $\operatorname{dn} 78$ & $\begin{array}{l}\text { PHENOBARBITAL } \\
\text { injection }\end{array}$ \\
\hline dn1y. & ACETAZOLAMIDE [EP] 250mg tablets & $\operatorname{dn} 72$. & PHENOBARBITAL 30mg tablets \\
\hline $\operatorname{dn} 1 z$. & $\begin{array}{l}\text { ACETAZOLAMIDE }[\mathrm{EP}] \quad 500 \mathrm{mg} \\
\text { injection }\end{array}$ & $\operatorname{dn} 73$. & PHENOBARBITAL 60mg tablets \\
\hline $\operatorname{dn} 1 x$. & $\begin{array}{l}\text { ACETAZOLAMIDE }[\mathrm{EP}] 500 \mathrm{mg} \mathrm{m} / \mathrm{r} \\
\text { capsules }\end{array}$ & $\operatorname{dn} 77$. & $\begin{array}{l}\text { PHENOBARBITONE } 15 \mathrm{mg} / 10 \mathrm{~mL} \\
\text { elixir }\end{array}$ \\
\hline $\operatorname{dn} 1 .$. & ACETAZOLAMIDE [EPILEPSY] & $\operatorname{dn} 75$ & $\begin{array}{l}\text { PHENOBARBITONE SODIUM } 30 \mathrm{mg} \\
\text { tablets }\end{array}$ \\
\hline do41. & ATIVAN $[\mathrm{EP}] 4 \mathrm{mg} / \mathrm{mL}$ injection & $\operatorname{dn} 76$ & $\begin{array}{l}\text { PHENOBARBITONE SODIUM } 60 \mathrm{mg} \\
\text { tablets }\end{array}$ \\
\hline dn3J. & CARBAGEN SR 200mg $\mathrm{m} / \mathrm{r}$ tablets & $\operatorname{dn} 8 .$. & PHENYTOIN \\
\hline
\end{tabular}


Table 6.2 AED Read codes - Continued ...

\begin{tabular}{|c|c|c|c|}
\hline Code & Description & Code & Description \\
\hline$\overline{\operatorname{dn} 3 K}$. & CARBAGEN SR 400mg $\mathrm{m} / \mathrm{r}$ tablets & $\operatorname{dn} 92$. & PHENYTOIN 100mg tablets \\
\hline $\operatorname{dn} 3 .$. & CARBAMAZEPINE & dn8y. & PHENYTOIN 30mg/5mL suspension \\
\hline dn3y. & $\begin{array}{l}\text { CARBAMAZEPINE 100mg chewable } \\
\text { tablets }\end{array}$ & $\operatorname{dn} 83$. & PHENYTOIN 50mg chewable tablets \\
\hline $\operatorname{dn} 31$. & CARBAMAZEPINE 100mg tablets & $\operatorname{dn} 91$. & PHENYTOIN 50mg tablets \\
\hline $\operatorname{dn} 3 z$. & $\begin{array}{l}\text { CARBAMAZEPINE } 100 \mathrm{mg} / 5 \mathrm{~mL} \text { sugar } \\
\text { free liquid }\end{array}$ & $\operatorname{dn} 8 z$ & $\begin{array}{l}\text { PHENYTOIN } 90 \mathrm{mg} / 5 \mathrm{~mL} \text { sugar free } \\
\text { suspension }\end{array}$ \\
\hline $\operatorname{dn} 3 \mathrm{v}$. & $\begin{array}{l}\text { CARBAMAZEPINE } \quad 125 \mathrm{mg} \\
\text { suppositories }\end{array}$ & $\operatorname{dn} 9 .$. & PHENYTOIN SODIUM \\
\hline $\operatorname{dn} 3 x$. & $\begin{array}{l}\text { CARBAMAZEPINE 200mg chewable } \\
\text { tablets }\end{array}$ & do6.. & $\begin{array}{l}\text { PHENYTOIN SODIUM } \\
\text { EPILEPST }]\end{array}$ \\
\hline dn3a. & CARBAMAZEPINE 200mg m/r tabs & $\operatorname{dn} 9 \mathrm{z}$. & $\begin{array}{l}\text { PHENYTOIN SODIUM } \\
\text { capsules }\end{array}$ \\
\hline $\operatorname{dn} 32$. & CARBAMAZEPINE 200mg tablets & do6z. & $\begin{array}{l}\text { PHENYTOIN SODIUM } 250 \mathrm{mg} / 5 \mathrm{~mL} \\
\text { injection }\end{array}$ \\
\hline $\operatorname{dn} 3 \mathrm{w}$. & $\begin{array}{l}\text { CARBAMAZEPINE } \\
\text { suppositories }\end{array}$ & $\operatorname{dn} 9 x$ & PHENYTOIN SODIUM 25mg caps \\
\hline dn3b. & CARBAMAZEPINE 400mg $\mathrm{m} / \mathrm{r}$ tabs & $\operatorname{dn} 9 w$. & $\begin{array}{l}\text { PHENYTOIN SODIUM } 300 \mathrm{mg} \\
\text { capsules }\end{array}$ \\
\hline $\operatorname{dn} 33$. & CARBAMAZEPINE 400mg tablets & dn9y. & PHENYTOIN SODIUM 50mg capsules \\
\hline dnc.. & CLOBAZAM [EPILEPSY ONLY] & dng.. & PIRACETAM \\
\hline do3.. & $\begin{array}{l}\text { CLOMETHIAZOLE EDISYLATE } \\
\text { [CENTRAL NERVOUS SYSTEM USE] }\end{array}$ & dng 5. & PIRACETAM $1.2 \mathrm{~g}$ tablets \\
\hline do3z. & $\begin{array}{l}\text { CLOMETHIAZOLE EDISYLATE } \\
8 \mathrm{mg} / \mathrm{mL} \text { intravenous infusion }\end{array}$ & $\operatorname{dng} 6$. & $\begin{array}{l}\text { PIRACETAM } \quad 333.3 \mathrm{mg} / \mathrm{mL} \\
\text { solution }\end{array}$ \\
\hline $\operatorname{dn} 4 .$. & $\begin{array}{l}\text { CLONAZEPAM } \\
\text { CONTROL] }\end{array}$ & $\operatorname{dng} 4$. & PIRACETAM 800mg tablets \\
\hline do2.. & CLONAZEPAM [STATUS EPILEPSY] & dnp.. & PREGABALIN \\
\hline $\operatorname{dn} 4 \mathrm{w}$. & $\begin{array}{l}\text { CLONAZEPAM } 0.5 \mathrm{mg} / 5 \mathrm{~mL} \text { sugar free } \\
\text { oral solution }\end{array}$ & dnpv. & PREGABALIN 100mg capsules \\
\hline do2z. & CLONAZEPAM 1mg/1mL injection & dnpw. & PREGABALIN 150mg capsules \\
\hline $\operatorname{dn} 4 \mathrm{z}$. & CLONAZEPAM 2mg tablets & dnpu. & PREGABALIN 200mg capsules \\
\hline $\operatorname{dn} 4 x$ & $\begin{array}{l}\text { CLONAZEPAM } 2 \mathrm{mg} / 5 \mathrm{~mL} \text { sugar free } \\
\text { oral solution }\end{array}$ & dnps. & PREGABALIN 225mg capsules \\
\hline $\operatorname{dn} 4 y$. & CLONAZEPAM 500microgram tablets & dnpz. & PREGABALIN 25mg capsules \\
\hline $\operatorname{dn} \ldots$ & CONTROL OF EPILEPSY & dnpt. & PREGABALIN 300mg capsules \\
\hline dnh1. & CONVULEX 150mg e/c capsules & dnpy. & PREGABALIN 50mg capsules \\
\hline $\operatorname{dnh} 2$. & CONVULEX 300mg e/c capsules & dnpx. & PREGABALIN $75 \mathrm{mg}$ capsules \\
\hline dnh3. & CONVULEX 500mg e/c capsules & dna.. & PRIMIDONE \\
\hline dnh7. & DEPAKOTE $250 \mathrm{mg}$ e/c tablets & dnay. & PRIMIDONE 250mg tablets \\
\hline $\operatorname{dnh} 8$. & DEPAKOTE 500mg e/c tablets & dnaz. & $\begin{array}{l}\text { PRIMIDONE } \quad 250 \mathrm{mg} / 5 \mathrm{~mL} \\
\text { suspension }\end{array}$ \\
\hline dns1. & DIACOMIT 250mg capsules & dnax. & PRIMIDONE 50mg tablets \\
\hline
\end{tabular}


Table 6.2 AED Read codes - Continued ...

\begin{tabular}{|c|c|c|c|}
\hline Code & Description & Code & Description \\
\hline dns3. & $\begin{array}{l}\text { DIACOMIT } 250 \mathrm{mg} / \text { sachet powder for } \\
\text { oral suspension }\end{array}$ & dni2. & $\begin{array}{l}\text { PRO-EPANUTIN } \\
\text { injection concentrate }\end{array}$ \\
\hline $\operatorname{dns} 2$. & DIACOMIT 500mg capsules & dnv.. & RETIGABINE \\
\hline dns4. & $\begin{array}{l}\text { DIACOMIT } 500 \mathrm{mg} / \text { sachet powder for } \\
\text { oral suspension }\end{array}$ & dnv8. & RETIGABINE 100mg tablets \\
\hline $\operatorname{dn} 12$. & DIAMOX [EP] 250mg tablets & dnv9. & RETIGABINE $200 \mathrm{mg}$ tablets \\
\hline $\operatorname{dn} 13$. & DIAMOX [EP] 500mg injection & dnvA. & RETIGABINE 300mg tablets \\
\hline $\operatorname{dn} 11$. & DIAMOX [EP] 500mg m/r capsules & dnvB. & RETIGABINE $400 \mathrm{mg}$ tablets \\
\hline do11. & DIAZEMULS [EP] 10mg/2mL injection & dnv7. & RETIGABINE 50mg tablets \\
\hline do1.. & DIAZEPAM [EPILEPSY USE] & dnvC. & $\begin{array}{l}\text { RETIGABINE } 50 \mathrm{mg}+100 \mathrm{mg} \text { tablets } \\
\text { initiation pack }\end{array}$ \\
\hline do1y. & $\begin{array}{l}\text { DIAZEPAM } 10 \mathrm{mg} / 2.5 \mathrm{~mL} \quad \text { rectal } \\
\text { solution }\end{array}$ & do21. & RIVOTRIL 1mg/1mL injection \\
\hline do19. & DIAZEPAM 10mg/2.5mL RecTubes & $\operatorname{dn} 42$. & RIVOTRIL $2 \mathrm{mg}$ tablets \\
\hline do1v. & $\begin{array}{l}\text { DIAZEPAM } 10 \mathrm{mg} / 2 \mathrm{~mL} \quad \text { emulsion } \\
\text { injection }\end{array}$ & $\operatorname{dn} 41$. & RIVOTRIL 500micrograms tablets \\
\hline do1w. & DIAZEPAM 10mg/2mL injection & dnr.. & RUFINAMIDE \\
\hline do1t. & $\begin{array}{l}\text { DIAZEPAM } 2.5 \mathrm{mg} / 1.25 \mathrm{~mL} \quad \text { rectal } \\
\text { solution }\end{array}$ & dnrz. & RUFINAMIDE 100mg tablets \\
\hline do1A. & DIAZEPAM $2.5 \mathrm{mg} / 1.25 \mathrm{~mL}$ RecTubes & dnry. & RUFINAMIDE $200 \mathrm{mg}$ tablets \\
\hline do1u. & DIAZEPAM $20 \mathrm{mg} / 5 \mathrm{~mL}$ rectal solution & dnrx. & RUFINAMIDE 400mg tablets \\
\hline do1x. & DIAZEPAM $5 \mathrm{mg} / 2.5 \mathrm{~mL}$ rectal solution & dne4. & SABRIL 500mg powder sachets \\
\hline do18. & DIAZEPAM 5mg/2.5mL RecTubes & dne2. & SABRIL 500mg tablets \\
\hline $\operatorname{dn} 54$. & EMESIDE $250 \mathrm{mg} / 5 \mathrm{~mL}$ syrup & dnb.. & SODIUM VALPROATE \\
\hline do61. & EPANUTIN [EP] $250 \mathrm{mg} / 5 \mathrm{~mL}$ injection & dnbv. & $\begin{array}{l}\text { SODIUM VALPROATE 100mg } \\
\text { crushable tablets }\end{array}$ \\
\hline dn95. & EPANUTIN 100mg capsules & dnbo. & $\begin{array}{l}\text { SODIUM VALPROATE } 100 \mathrm{mg} / \text { sachet } \\
\mathrm{m} / \mathrm{r} \text { granules }\end{array}$ \\
\hline $\operatorname{dn} 93$. & EPANUTIN 25mg capsules & dnbJ. & $\begin{array}{l}\text { SODIUM VALPROATE } 150 \mathrm{mg} \mathrm{m} / \mathrm{r} \\
\text { capsules }\end{array}$ \\
\hline $\operatorname{dn} 96$. & EPANUTIN 300mg capsules & dnbN. & $\begin{array}{l}\text { SODIUM VALPROATE } 1 \mathrm{~g} / 10 \mathrm{~mL} \\
\text { solution for injection }\end{array}$ \\
\hline $\operatorname{dn} 81$. & EPANUTIN $30 \mathrm{mg} / 5 \mathrm{~mL}$ suspension & dnbM. & $\begin{array}{l}\text { SODIUM VALPROATE } 1 \mathrm{~g} / \text { sachet } \mathrm{m} / \mathrm{r} \\
\text { granules }\end{array}$ \\
\hline dn94. & EPANUTIN 50mg capsules & dnbw. & $\begin{array}{l}\text { SODIUM VALPROATE } 200 \mathrm{mg} \\
\text { crushable tablets }\end{array}$ \\
\hline $\operatorname{dn} 82$. & EPANUTIN 50mg Infatabs & dnb7. & $\begin{array}{l}\text { SODIUM VALPROATE } 200 \mathrm{mg} \mathrm{e} / \mathrm{c} \\
\text { tablets }\end{array}$ \\
\hline dnb1. & EPILIM 100mg crushable tablets & dnbr. & $\begin{array}{l}\text { SODIUM VALPROATE } 200 \mathrm{mg} \mathrm{m} / \mathrm{r} \\
\text { tablets }\end{array}$ \\
\hline dnb2. & EPILIM 200mg e/c tablets & dnby. & $\begin{array}{l}\text { SODIUM VALPROATE } 200 \mathrm{mg} / 5 \mathrm{~mL} \\
\text { sugar free liquid }\end{array}$ \\
\hline dnb4. & EPILIM $200 \mathrm{mg} / 5 \mathrm{~mL}$ sugar free liquid & dnbz. & $\begin{array}{l}\text { SODIUM VALPROATE } 200 \mathrm{mg} / 5 \mathrm{~mL} \\
\text { syrup }\end{array}$ \\
\hline
\end{tabular}


Table 6.2 AED Read codes - Continued ...

\begin{tabular}{|c|c|c|c|}
\hline Code & Description & Code & Description \\
\hline dnb5. & EPILIM $200 \mathrm{mg} / 5 \mathrm{~mL}$ syrup & dnbp. & $\begin{array}{l}\text { SODIUM VALPROATE } 250 \mathrm{mg} / \text { sachet } \\
\mathrm{m} / \mathrm{r} \text { granules }\end{array}$ \\
\hline dnb3. & EPILIM 500mg e/c tablets & dnbK. & $\begin{array}{l}\text { SODIUM VALPROATE } 300 \mathrm{mg} \mathrm{m} / \mathrm{r} \\
\text { capsules }\end{array}$ \\
\hline dnbc. & EPILIM CHRONO $200 \mathrm{~m} / \mathrm{r}$ tablets & dnbs. & $\begin{array}{l}\text { SODIUM VALPROATE } 300 \mathrm{mg} \mathrm{m} / \mathrm{r} \\
\text { tablets }\end{array}$ \\
\hline dnbd. & EPILIM CHRONO $300 \mathrm{~m} / \mathrm{r}$ tablets & dnbE. & $\begin{array}{l}\text { SODIUM VALPROATE } 300 \mathrm{mg} / 3 \mathrm{~mL} \\
\text { solution for injection }\end{array}$ \\
\hline dnbe. & EPILIM CHRONO $500 \mathrm{~m} / \mathrm{r}$ tablets & dnbu. & $\begin{array}{l}\text { SODIUM VALPROATE } 400 \mathrm{mg} / 4 \mathrm{~mL} \\
\text { injection }\end{array}$ \\
\hline dnbQ. & $\begin{array}{l}\text { EPILIM CHRONOSPHERE } \\
100 \mathrm{mg} / \text { sachet } \mathrm{m} / \mathrm{r} \text { granules }\end{array}$ & dnb8. & $\begin{array}{l}\text { SODIUM VALPROATE } 500 \mathrm{mg} \text { e/c } \\
\text { tablets }\end{array}$ \\
\hline dnbU. & $\begin{array}{l}\text { EPILIM CHRONOSPHERE } 1 \mathrm{~g} / \text { sachet } \\
\mathrm{m} / \mathrm{r} \text { granules }\end{array}$ & dnbt. & $\begin{array}{l}\text { SODIUM VALPROATE } 500 \mathrm{mg} \mathrm{m} / \mathrm{r} \\
\text { tablets }\end{array}$ \\
\hline dnbR. & $\begin{array}{l}\text { EPILIM } \quad \text { CHRONOSPHERE } \\
250 \mathrm{mg} / \text { sachet } \mathrm{m} / \mathrm{r} \text { granules }\end{array}$ & dnbx. & SODIUM VALPROATE 500mg tablets \\
\hline dnbS. & $\begin{array}{l}\text { EPILIM } \quad \text { CHRONOSPHERE } \\
500 \mathrm{mg} / \text { sachet } \mathrm{m} / \mathrm{r} \text { granules }\end{array}$ & dnbL. & $\begin{array}{l}\text { SODIUM VALPROATE } 500 \mathrm{mg} / \mathrm{sachet} \\
\mathrm{m} / \mathrm{r} \text { granules }\end{array}$ \\
\hline dnbP. & $\begin{array}{l}\text { EPILIM CHRONOSPHERE } \\
50 \mathrm{mg} / \text { sachet } \mathrm{m} / \mathrm{r} \text { granules }\end{array}$ & dnbn. & $\begin{array}{l}\text { SODIUM VALPROATE } 50 \mathrm{mg} / \mathrm{sachet} \\
\mathrm{m} / \mathrm{r} \text { granules }\end{array}$ \\
\hline dnbT. & $\begin{array}{l}\text { EPILIM CHRONOSPHERE } \\
750 \mathrm{mg} / \text { sachet } \mathrm{m} / \mathrm{r} \text { granules }\end{array}$ & dnbq. & $\begin{array}{l}\text { SODIUM VALPROATE } 750 \mathrm{mg} / \mathrm{sach} \text { t } \\
\mathrm{m} / \mathrm{r} \text { granules }\end{array}$ \\
\hline dnb6. & EPILIM IV 400mg/4mL injection & do... & STATUS EPILEPTICUS DRUGS \\
\hline dnbF. & EPISENTA 150mg m/r capsules & do12. & STESOLID [EP] $10 \mathrm{mg} / 2 \mathrm{~mL}$ injection \\
\hline dnbO. & $\begin{array}{l}\text { EPISENTA } 1 \mathrm{~g} / 10 \mathrm{~mL} \text { solution for } \\
\text { injection }\end{array}$ & do15. & STESOLID $10 \mathrm{mg} / 2.5 \mathrm{~mL}$ rectal solution \\
\hline dnbI. & EPISENTA $1 \mathrm{~g} /$ sachet $\mathrm{m} / \mathrm{r}$ granules & do14. & STESOLID $5 \mathrm{mg} / 2.5 \mathrm{~mL}$ rectal solution \\
\hline dnbG. & EPISENTA 300mg m/r capsules & dnsw. & $\begin{array}{l}\text { STIRIPENDOL } 500 \mathrm{mg} / \text { sachet powder } \\
\text { for oral suspension }\end{array}$ \\
\hline dnbD. & $\begin{array}{l}\text { EPISENTA } 300 \mathrm{mg} / 3 \mathrm{~mL} \text { solution for } \\
\text { injection }\end{array}$ & dns.. & STIRIPENTOL \\
\hline $\begin{array}{l}\text { dnbH. } \\
\text { dnbB. }\end{array}$ & $\begin{array}{l}\text { EPISENTA } 500 \mathrm{mg} / \text { sachet } \mathrm{m} / \mathrm{r} \text { granules } \\
\text { EPIVAL CR } 300 \mathrm{mg} \mathrm{m} / \mathrm{r} \text { tablets }\end{array}$ & $\begin{array}{l}\text { dnsz. } \\
\text { dnsx. }\end{array}$ & $\begin{array}{l}\text { STIRIPENTOL } 250 \mathrm{mg} \text { capsules } \\
\text { STIRIPENTOL } 250 \mathrm{mg} / \text { sachet powder } \\
\text { for oral suspension }\end{array}$ \\
\hline dnbC. & EPIVAL CR 500mg m/r tablets & dnsy. & STIRIPENTOL 500mg capsules \\
\hline dnu.. & ESLICARBAZEPINE & $\operatorname{dn} 3 c$. & TEGRETOL 100mg chewable tablets \\
\hline dnu2. & $\begin{array}{l}\text { ESLICARBAZEPINE } \\
\text { 800mg tablets }\end{array}$ & $\operatorname{dn} 34$ & TEGRETOL 100mg tablets \\
\hline $\operatorname{dn} 5 .$. & ETHOSUXIMIDE & $\operatorname{dn} 37$. & $\begin{array}{l}\text { TEGRETOL } 100 \mathrm{mg} / 5 \mathrm{~mL} \text { sugar free } \\
\text { liquid }\end{array}$ \\
\hline $\operatorname{dn} 5 x$ & ETHOSUXIMIDE 250mg capsules & $\mathrm{dn} 3 \mathrm{D}$. & TEGRETOL 125mg suppositories \\
\hline $\operatorname{dn} 5 z$ & ETHOSUXIMIDE $250 \mathrm{mg} / 5 \mathrm{~mL}$ syrup & $\operatorname{dn} 3 \mathrm{~d}$ & TEGRETOL 200mg chewable tablets \\
\hline dni.. & FOSPHENYTOIN SODIUM & $\operatorname{dn} 35$ & TEGRETOL 200mg tablets \\
\hline
\end{tabular}


Table 6.2 AED Read codes - Continued ...

\begin{tabular}{|c|c|c|c|}
\hline Code & Description & Code & Description \\
\hline \multirow[t]{2}{*}{ dni1. } & $\begin{array}{ll}\text { FOSPHENYTOIN } & \text { SODIUM }\end{array}$ & $\operatorname{dn} 3 \mathrm{E}$. & TEGRETOL 250mg suppositories \\
\hline & 750mg/10mL injection concentrate & & \\
\hline dnj.. & GABAPENTIN & $\operatorname{dn} 36$. & TEGRETOL 400mg tablets \\
\hline dnj1. & GABAPENTIN 100mg capsules & $\operatorname{dn} 38$. & TEGRETOL RETARD 200mg m/r tabs \\
\hline dnj2. & GABAPENTIN 300mg capsules & $\operatorname{dn} 39$. & TEGRETOL RETARD 400mg m/r tabs \\
\hline dnjx. & $\begin{array}{l}\text { GABAPENTIN } 300 \mathrm{mg} \text { capsules } / 600 \mathrm{mg} \\
\text { tablets titration pack }\end{array}$ & dnl.. & TIAGABINE \\
\hline dnj3. & GABAPENTIN 400mg capsules & dnl2. & TIAGABINE 10mg tablets \\
\hline dnjy. & GABAPENTIN 600mg tablets & dnl3. & TIAGABINE $15 \mathrm{mg}$ tablets \\
\hline dnjz. & GABAPENTIN $800 \mathrm{mg}$ tablets & dnl1. & TIAGABINE 5mg tablets \\
\hline dnl5. & GABITRIL 10mg tablets & $\operatorname{dn} 3 \mathrm{~F}$. & TIMONIL RETARD 200mg $\mathrm{m} / \mathrm{r}$ tablets \\
\hline dnl6. & GABITRIL 15mg tablets & dn3G. & TIMONIL RETARD 400mg $\mathrm{m} / \mathrm{r}$ tablets \\
\hline dnl4. & GABITRIL 5mg tablets & dnk5. & TOPAMAX 100mg tablets \\
\hline \multirow[t]{2}{*}{ do31. } & HEMINEVRIN $\quad$ [CNS] & $\operatorname{dnk} 6$. & TOPAMAX 200mg tablets \\
\hline & intravenous infusion & & \\
\hline dnr1. & INOVELON 100mg tablets & $\operatorname{dnk} 8$ & TOPAMAX 25mg tablets \\
\hline dnr2. & INOVELON 200mg tablets & dnk4. & TOPAMAX 50mg tablets \\
\hline dnr3. & INOVELON 400mg tablets & dnkB. & TOPAMAX SPRINKLE 15mg capsules \\
\hline dno5. & KEPPRA $100 \mathrm{mg} / \mathrm{mL} \mathrm{s} / \mathrm{f}$ oral solution & dnkC. & TOPAMAX SPRINKLE 25mg capsules \\
\hline dno3. & KEPPRA $1 \mathrm{~g}$ tablets & dnkE. & TOPAMAX SPRINKLE 50mg capsules \\
\hline dno1. & KEPPRA $250 \mathrm{mg}$ tablets & dnk.. & TOPIRAMATE \\
\hline dno2. & KEPPRA 500mg tablets & $\operatorname{dnk} 2$. & TOPIRAMATE 100mg tablets \\
\hline dno6. & $\begin{array}{l}\text { KEPPRA } 500 \mathrm{mg} / 5 \mathrm{~mL} \text { solution for } \\
\text { injection }\end{array}$ & dnk9. & TOPIRAMATE $15 \mathrm{mg}$ beads in capsules \\
\hline dno4. & KEPPRA 750mg tablets & $\operatorname{dnk} 3$. & TOPIRAMATE 200mg tablets \\
\hline dnt.. & LACOSAMIDE & dnkA. & TOPIRAMATE $25 \mathrm{mg}$ beads in capsules \\
\hline $\operatorname{dnt} A$. & LACOSAMIDE $100 \mathrm{mg}$ tablets & dnk7. & TOPIRAMATE 25mg tablets \\
\hline dntB. & LACOSAMIDE $150 \mathrm{mg}$ tablets & dnkD. & TOPIRAMATE $50 \mathrm{mg}$ beads in capsules \\
\hline $\operatorname{dnt} 8$. & $\begin{array}{l}\text { LACOSAMIDE } 15 \mathrm{mg} / 1 \mathrm{~mL} \text { sugar free } \\
\text { liquid }\end{array}$ & dnk1. & TOPIRAMATE 50mg tablets \\
\hline dntC. & LACOSAMIDE 200mg tablets & dnm1. & TRILEPTAL 150 tablets \\
\hline dnt7. & $\begin{array}{l}\text { LACOSAMIDE } 200 \mathrm{mg} / 20 \mathrm{~mL} \text { solution } \\
\text { for injection }\end{array}$ & dnm2. & TRILEPTAL 300 tablets \\
\hline dnt9. & LACOSAMIDE 50mg tablets & dnm3. & TRILEPTAL 600 tablets \\
\hline dnf9. & LAMICTAL 100mg dispersible tablets & dnm4. & $\begin{array}{l}\text { TRILEPTAL } 60 \mathrm{mg} / \mathrm{mL} \text { sugar free oral } \\
\text { suspension }\end{array}$ \\
\hline dnf4. & LAMICTAL 100mg tablets & dnv2. & TROBALT 100mg tablets \\
\hline dnfD. & LAMICTAL 200mg tablets & dnv3. & TROBALT 200mg tablets \\
\hline dnf8. & LAMICTAL $25 \mathrm{mg}$ dispersible tablets & dnv4. & TROBALT 300mg tablets \\
\hline $\operatorname{dnf} 6$. & LAMICTAL 25mg tablets & dnv5. & TROBALT 400mg tablets \\
\hline dnfJ. & LAMICTAL 2mg dispersible tablets & dnv1. & TROBALT 50mg tablets \\
\hline dnf3. & LAMICTAL 50mg tablets & dnv6. & TROBALT tablets initiation pack \\
\hline dnf7. & LAMICTAL 5mg dispersible tablets & do16. & VALIUM [EP] $10 \mathrm{mg} / 2 \mathrm{~mL}$ injection \\
\hline
\end{tabular}


Table 6.2 AED Read codes - Continued ...

\begin{tabular}{|c|c|c|c|}
\hline Code & Description & Code & Description \\
\hline $\operatorname{dnfF}$. & $\begin{array}{l}\text { LAMICTAL MONOTHERAPY } 25 \mathrm{mg} \\
\text { starter pack }\end{array}$ & do17. & VALIUM [EP] $20 \mathrm{mg} / 4 \mathrm{~mL}$ injection \\
\hline dnfH. & $\begin{array}{l}\text { LAMICTAL NON-VALPROATE } \\
\text { ADD-ON 50mg starter pack }\end{array}$ & dnh.. & VALPROIC ACID \\
\hline dnfG. & $\begin{array}{l}\text { LAMICTAL VALPROATE ADD-ON } \\
25 \mathrm{mg} \text { starter pack }\end{array}$ & $\operatorname{dnh} 4$. & VALPROIC ACID 150mg e/c capsules \\
\hline dnf.. & LAMOTRIGINE & dnhz. & VALPROIC ACID $250 \mathrm{mg}$ e/c tablets \\
\hline dnfC. & $\begin{array}{l}\text { LAMOTRIGINE } 100 \mathrm{mg} \text { dispersible } \\
\text { tablets }\end{array}$ & $\operatorname{dnh} 5$. & VALPROIC ACID 300mg e/c capsules \\
\hline dnf2. & LAMOTRIGINE 100mg tablets & $\operatorname{dnh} 6$. & VALPROIC ACID 500mg e/c capsules \\
\hline dnfE. & LAMOTRIGINE $200 \mathrm{mg}$ tablets & dnhy. & VALPROIC ACID 500mg e/c tablets \\
\hline dnfB. & $\begin{array}{l}\text { LAMOTRIGINE } 25 \mathrm{mg} \text { dispersible } \\
\text { tablets }\end{array}$ & dne.. & VIGABATRIN \\
\hline dnf5. & LAMOTRIGINE 25mg tablets & dne3. & VIGABATRIN 500mg powder sachets \\
\hline dnfz. & $\begin{array}{l}\text { LAMOTRIGINE } 2 \mathrm{mg} \text { dispersible } \\
\text { tablets }\end{array}$ & dne1. & VIGABATRIN 500mg tablets \\
\hline dnf1. & LAMOTRIGINE 50mg tablets & dnt4. & VIMPAT 100mg tablets \\
\hline dnfA. & $\begin{array}{l}\text { LAMOTRIGINE } 5 \mathrm{mg} \text { dispersible } \\
\text { tablets }\end{array}$ & dnt5. & VIMPAT 150mg tablets \\
\hline dno.. & LEVETIRACETAM & dnt2. & VIMPAT $15 \mathrm{mg} / 1 \mathrm{~mL}$ sugar free liquid \\
\hline dnov. & $\begin{array}{l}\text { LEVETIRACETAM } 100 \mathrm{mg} / \mathrm{mL} \text { s/f oral } \\
\text { solution }\end{array}$ & dnt6. & VIMPAT 200mg tablets \\
\hline dnox. & LEVETIRACETAM 1g tablets & dnt1. & $\begin{array}{l}\text { VIMPAT } 200 \mathrm{mg} / 20 \mathrm{~mL} \text { solution for } \\
\text { injection }\end{array}$ \\
\hline dnoz. & LEVETIRACETAM 250mg tablets & dnt3. & VIMPAT 50mg tablets \\
\hline dnoy. & LEVETIRACETAM 500mg tablets & $\operatorname{dn} 56$. & ZARONTIN $250 \mathrm{mg} / 5 \mathrm{~mL}$ syrup \\
\hline dnou. & $\begin{array}{l}\text { LEVETIRACETAM } \\
\text { solution for injection }\end{array}$ & dnu1. & ZEBINIX 800mg tablets \\
\hline dnow. & LEVETIRACETAM 750mg tablets & dnq6. & ZONEGRAN 100mg capsules \\
\hline do4.. & LORAZEPAM [EPILEPSY] & dnq4. & ZONEGRAN 25mg capsules \\
\hline dnp4. & LYRICA 100mg capsules & dnq5. & ZONEGRAN 50mg capsules \\
\hline dnp5. & LYRICA 150mg capsules & dnq.. & ZONISAMIDE \\
\hline dnp6. & LYRICA 200mg capsules & dnq3. & ZONISAMIDE 100mg capsules \\
\hline dnp8. & LYRICA $225 \mathrm{mg}$ capsules & dnq1. & ZONISAMIDE 25mg capsules \\
\hline dnp1. & LYRICA $25 \mathrm{mg}$ capsules & dnq2. & ZONISAMIDE 50mg capsule \\
\hline
\end{tabular}




\section{Bibliography}

[1] W Owen Pickrell, Arron S Lacey, Rhys H Thomas, Ronan A Lyons, Phil EM Smith, and Mark I Rees. Trends in the first antiepileptic drug prescribed for epilepsy between 2000 and 2010. Seizure-European Journal of Epilepsy, 23(1): $77-80,2014$.

[2] Erik F Tjong Kim Sang and Sabine Buchholz. Introduction to the conll-2000 shared task: Chunking. In Proceedings of the 2nd workshop on Learning language in logic and the 4 th conference on Computational natural language learning-Volume 7, pages 127-132. Association for Computational Linguistics, 2000.

[3] Arron S Lacey, William Owen Pickrell, Rhys H Thomas, Mike P Kerr, Cathy P White, and Mark I Rees. Educational attainment of children born to mothers with epilepsy. J Neurol Neurosurg Psychiatry, pages jnnp-2017, 2018.

[4] Owen Pickrell, Arron Lacey, Owen Bodger, Joanne Demmler, Rhys Thomas, Ronan Lyons, Phil Smith, Mark Rees, and Mike Kerr. Epilepsy prevalence, incidence and socioeconomic deprivation. Accepted into Epilepsia, 2014.

[5] Beata Fonferko-Shadrach, Arron S Lacey, Catharine P White, HW Rob Powell, Inder MS Sawhney, Ronan A Lyons, Phil EM Smith, Mike P Kerr, Mark I Rees, and W Owen Pickrell. Validating epilepsy diagnoses in routinely collected data. Seizure-European Journal of Epilepsy, 52:195-198, 2017.

[6] Anthony K Ngugi, Christian Bottomley, Immo Kleinschmidt, Josemir W Sander, and Charles R Newton. Estimation of the burden of active and life-time epilepsy: a meta-analytic approach. Epilepsia, 51(5):883-890, 2010.

[7] JW Sander and SD Shorvon. Epidemiology of the epilepsies. Journal of neurology, neurosurgery, and psychiatry, 61(5):433, 1996.

[8] Matilde Leonardi and T Bedirhan Ustun. The global burden of epilepsy. Epilepsia, 43(s6):21-25, 2002. 
[9] Robert S Fisher, Carlos Acevedo, Alexis Arzimanoglou, Alicia Bogacz, J Helen Cross, Christian E Elger, Jerome Engel, Lars Forsgren, Jacqueline A French, Mike Glynn, et al. Ilae official report: a practical clinical definition of epilepsy. Epilepsia, 55(4):475-482, 2014.

[10] Anne T Berg, Samuel F Berkovic, Martin J Brodie, Jeffrey Buchhalter, J Helen Cross, Walter van Emde Boas, Jerome Engel, Jacqueline French, Tracy A Glauser, Gary W Mathern, et al. Revised terminology and concepts for organization of seizures and epilepsies: report of the ilae commission on classification and terminology, 2005-2009. Epilepsia, 51(4):676-685, 2010.

[11] Jerome Engel Jr. Ilae classification of epilepsy syndromes. Epilepsy research, 70:5-10, 2006.

[12] Anna L Peljto, Christie Barker-Cummings, Vincent M Vasoli, Cynthia L Leibson, W Allen Hauser, Jeffrey R Buchhalter, and Ruth Ottman. Familial risk of epilepsy: a population-based study. Brain, 137(3):795-805, 2014.

[13] D Janz, G Beck-Mannagetta, and T Sander. Do idiopathic generalized epilepsies share a common susceptibility gene? Neurology, 42(4 Suppl 5):48-55, 1992.

[14] Samuel F Berkovic, R Anne Howell, David A Hay, and John L Hopper. Epilepsies in twins: genetics of the major epilepsy syndromes. Annals of neurology, 43(4): 435-445, 1998.

[15] G Beck-Mannagetta and D Janz. Syndrome-related genetics in generalized epilepsy. Epilepsy research. Supplement, 4:105, 1991.

[16] Patrick Cossette, Lidong Liu, Katéri Brisebois, Haiheng Dong, Anne Lortie, Michel Vanasse, Jean-Marc Saint-Hilaire, Lionel Carmant, Andrei Verner, Wei-Yang Lu, et al. Mutation of gabra1 in an autosomal dominant form of juvenile myoclonic epilepsy. Nature genetics, 31(2):184-189, 2002.

[17] Deb K Pal and David A Greenberg. Major susceptibility genes for common idiopathic epilepsies: Elp4 in rolandic epilepsy and brd2 in juvenile myoclonic epilepsy. 2012.

[18] John C Mulley, Ingrid E Scheffer, Steven Petrou, Leanne M Dibbens, Samuel F Berkovic, and Louise A Harkin. Scn1a mutations and epilepsy. Human mutation, 25(6):535-542, 2005.

[19] Louise A Harkin, Jacinta M McMahon, Xenia Iona, Leanne Dibbens, James T Pelekanos, Sameer M Zuberi, Lynette G Sadleir, Eva Andermann, Deepak 
Gill, Kevin Farrell, et al. The spectrum of scn1a-related infantile epileptic encephalopathies. Brain, 130(3):843-852, 2007.

[20] Samuel Steer, William O Pickrell, Michael P Kerr, and Rhys H Thomas. Epilepsy prevalence and socioeconomic deprivation in england. Epilepsia, 55 (10):1634-1641, 2014.

[21] Charles R Newton and Hector H Garcia. Epilepsy in poor regions of the world. The Lancet, 380(9848):1193-1201, 2012.

[22] Elias Olafsson and W Allen Hauser. Prevalence of epilepsy in rural iceland: A population-based study. Epilepsia, 40(11):1529-1534, 1999.

[23] MICHAEL J McLEAN and ROBERT L Macdonald. Sodium valproate, but not ethosuximide, produces use-and voltage-dependent limitation of high frequency repetitive firing of action potentials of mouse central neurons in cell culture. Journal of Pharmacology and Experimental therapeutics, 237(3):1001-1011, 1986.

[24] Cynthia L Harden. New antiepileptic drugs. Neurology, 44(5):787-787, 1994.

[25] Marc A Dichter and Martin J Brodie. New antiepileptic drugs. New England Journal of Medicine, 334(24):1583-1590, 1996.

[26] Anthony G Marson, Asya M Al-Kharusi, Muna Alwaidh, Richard Appleton, Gus A Baker, David W Chadwick, Celia Cramp, Oliver C Cockerell, Paul N Cooper, Julie Doughty, et al. The sanad study of effectiveness of valproate, lamotrigine, or topiramate for generalised and unclassifiable epilepsy: an unblinded randomised controlled trial. The Lancet, 369(9566):1016-1026, 2007.

[27] Kimford J Meador, Gus A Baker, Nancy Browning, Jill Clayton-Smith, Deborah T Combs-Cantrell, Morris Cohen, Laura A Kalayjian, Andres Kanner, Joyce D Liporace, Page B Pennell, et al. Cognitive function at 3 years of age after fetal exposure to antiepileptic drugs. New England Journal of Medicine, 360(16):1597-1605, 2009.

[28] Carol S Camfield, Sheila Chaplin, Anna-Beth Doyle, Stanley H Shapiro, Carl Cummings, and Peter R Camfield. Side effects of phenobarbital in toddlers; behavioral and cognitive aspects. The Journal of pediatrics, 95(3):361-365, 1979.

[29] TA Ketter, RM Post, and WH Theodore. Positive and negative psychiatric effects of antiepileptic drugs in patients with seizure disorders. Neurology, 53(5 Suppl 2):S53-67, 1999. 
[30] Hanne Dinesen, Lennart Gram, Teis Andersen, and Mogens Dam. Weight gain during treatment with valproate. Acta neurologica scandinavica, 70(2):65-69, 1984.

[31] William Owen Pickrell, Arron S Lacey, Rhys H Thomas, Philip EM Smith, and Mark I Rees. Weight change associated with antiepileptic drugs. J Neurol Neurosurg Psychiatry, 84(7):796-799, 2013.

[32] Rebekah Shallcross, Rebecca L Bromley, B Irwin, LJ Bonnett, J Morrow, and GA Baker. Child development following in utero exposure levetiracetam vs sodium valproate. Neurology, 76(4):383-389, 2011.

[33] Lars Skou Elkjær, Bodil Hammer Bech, Yuelian Sun, Thomas Munk Laursen, and Jakob Christensen. Association between prenatal valproate exposure and performance on standardized language and mathematics tests in school-aged children. JAMA neurology, 2018.

[34] Christopher JL Murray, Theo Vos, Rafael Lozano, Mohsen Naghavi, Abraham D Flaxman, Catherine Michaud, Majid Ezzati, Kenji Shibuya, Joshua A Salomon, Safa Abdalla, et al. Disability-adjusted life years (dalys) for 291 diseases and injuries in 21 regions, 1990-2010: a systematic analysis for the global burden of disease study 2010. The lancet, 380(9859):2197-2223, 2012.

[35] Hanneke M De Boer. "out of the shadows": a global campaign against epilepsy. Epilepsia, 43(s6):7-8, 2002.

[36] SD Lhatoo and JWAS Sander. The epidemiology of epilepsy and learning disability. Epilepsia, 42(s1):6-9, 2001.

[37] Gregory Stores. School-children with epilepsy at risk for learning and behaviour problems. Developmental Medicine $\&$ Child Neurology, 20(4):502-508, 1978.

[38] Joan K Austin, David W Dunn, Cynthia S Johnson, and Susan M Perkins. Behavioral issues involving children and adolescents with epilepsy and the impact of their families: recent research data. Epilepsy $\&$ Behavior, 5:33-41, 2004.

[39] Lorie D Hamiwka, G Yu Cara, Lorraine A Hamiwka, Elisabeth MS Sherman, Blaire Anderson, and Elaine Wirrell. Are children with epilepsy at greater risk for bullying than their peers? Epilepsy \& Behavior, 15(4):500-505, 2009.

[40] Irene M Elliott, Lucyna Lach, and Mary Lou Smith. I just want to be normal: a qualitative study exploring how children and adolescents view the impact of 
intractable epilepsy on their quality of life. Epilepsy $\&$ behavior, 7(4):664-678, 2005.

[41] Michael Seidenberg, Niels Beck, Michael Geisser, Bruno Giordani, J Chris Sackellares, Stanley Berent, FE Dreifuss, and Thomas J Boll. Academic achievement of children with epilepsy. Epilepsia, 27(6):753-759, 1986.

[42] R Schulz, Hans Otto Lüders, S Noachtar, T May, A Sakamoto, H Holthausen, and P Wolf. Amnesia of the epileptic aura. Neurology, 45(2):231-235, 1995.

[43] H Meinardi, RA Scott, R Reis, JWAS Sander, ILAE Commission on the Developing World, et al. The treatment gap in epilepsy: the current situation and ways forward. Epilepsia, 42(1):136-149, 2001.

[44] Rajendra Kale. Bringing epilepsy out of the shadows. BMJ: British Medical Journal, 315(7099):2, 1997.

[45] World Health Organization et al. Atlas: epilepsy care in the world. 2005.

[46] Joan Petersilia. Invisible victims-violence against persons with developmental disabilities. Hum. Rts., 27:9, 2000.

[47] Kate Jacoby and Ann Jacoby. Epilepsy and insurance in the uk: an exploratory survey of the experiences of people with epilepsy. Epilepsy $\&$ Behavior, 5(6): 884-893, 2004.

[48] Ann Jacoby, Joanne Gorry, and Gus A Baker. Employers' attitudes to employment of people with epilepsy: still the same old story? Epilepsia, 46(12):1978-1987, 2005.

[49] Dale C Hesdorffer, Hong Tian, Kishlay Anand, W Allen Hauser, Petur Ludvigsson, Elias Olafsson, and Olafur Kjartansson. Socioeconomic status is a risk factor for epilepsy in icelandic adults but not in children. Epilepsia, 46 (8):1297-1303, 2005.

[50] Christopher LI Morgan, Zahir Ahmed, and Michael P Kerr. Social deprivation and prevalence of epilepsy and associated health usage. Journal of Neurology, Neurosurgery \& Psychiatry, 69(1):13-17, 2000.

[51] Dominic C Heaney, Bridget K MacDonald, Alex Everitt, Simon Stevenson, Giovanni S Leonardi, Paul Wilkinson, and Josemir W Sander. Socioeconomic variation in incidence of epilepsy: prospective community based study in south east england. Bmj, 325(7371):1013-1016, 2002.

[52] Debbie A Lawlor, George Davey Smith, Rita Patel, and Shah Ebrahim. 
Life-course socioeconomic position, area deprivation, and coronary heart disease: findings from the british women's heart and health study. American journal of public health, 95(1):91-97, 2005.

[53] Chien-Chang Liao, Huai-Chia Chang, Chun-Chieh Yeh, Yi-Chun Chou, Wen-Ta Chiu, and Ta-Liang Chen. Socioeconomic deprivation and associated risk factors of traumatic brain injury in children. Journal of trauma and acute care surgery, 73(5):1327-1331, 2012.

[54] L Anne Jeffreys, Andrew L Clark, and Marek Koperski. Practice nurses' workload and consultation patterns. Br J Gen Pract, 45(397):415-418, 1995.

[55] New clinical classification system will streamline computerised medical records. 1990.

[56] World Health Organization et al. History of the development of the icd. World Health Organization, 2006.

[57] World Health Organization. International statistical classification of diseases and related health problems. 1, 2004.

[58] Stephen J Gillam, A Niroshan Siriwardena, and Nicholas Steel. Pay-for-performance in the united kingdom: impact of the quality and outcomes framework - a systematic review. The Annals of Family Medicine, 10(5):461-468, 2012.

[59] Melanie Calvert, Aparna Shankar, Richard J McManus, Helen Lester, and Nick Freemantle. Effect of the quality and outcomes framework on diabetes care in the united kingdom: retrospective cohort study. Bmj, 338:b1870, 2009.

[60] Congenital Anomaly Register. Information service for wales (caris). CARIS review 2012, 1998.

[61] Ronan A Lyons, Kerina H Jones, Gareth John, Caroline J Brooks, Jean-Philippe Verplancke, David V Ford, Ginevra Brown, and Ken Leake. The sail databank: linking multiple health and social care datasets. BMC Medical Informatics and Decision Making, 9(1):3, 2009.

[62] David V Ford, Kerina H Jones, Jean-Philippe Verplancke, Ronan A Lyons, Gareth John, Ginevra Brown, Caroline J Brooks, Simon Thompson, Owen Bodger, Tony Couch, et al. The sail databank: building a national architecture for e-health research and evaluation. BMC health services research, 9(1):157, 2009. 
[63] M Hyatt, SE Rodgers, S Paranjothy, D Fone, and RA Lyons. The wales electronic cohort for children (wecc) study. Archives of Disease in Childhood-Fetal and Neonatal Edition, 96(Suppl 1):Fa18-Fa18, 2011.

[64] Shantini Paranjothy, Frank Dunstan, William J Watkins, Melanie Hyatt, Joanne C Demmler, Ronan A Lyons, and David Fone. Gestational age, birth weight, and risk of respiratory hospital admission in childhood. Pediatrics, 132 (6):e1562-e1569, 2013.

[65] Belinda J Gabbe, Caroline Brooks, Joanne C Demmler, Steven Macey, Melanie A Hyatt, and Ronan A Lyons. The association between hospitalisation for childhood head injury and academic performance: evidence from a population e-cohort study. J Epidemiol Community Health, pages jech-2013, 2014.

[66] Shantini Paranjothy, Annette Evans, Amrita Bandyopadhyay, David Fone, Behnaz Schofield, Ann John, Mark A Bellis, Ronan A Lyons, Daniel Farewell, and Sara Jayne Long. Risk of emergency hospital admission in children associated with mental disorders and alcohol misuse in the household: an electronic birth cohort study. The Lancet Public Health, 2018.

[67] Narayan P Iyer, David F Tucker, Selwyn H Roberts, Marsham Moselhi, Margery Morgan, and Jean W Matthes. Outcome of fetuses with turner syndrome: a 10-year congenital anomaly register based study. The Journal of Maternal-Fetal \& Neonatal Medicine, 25(1):68-73, 2012.

[68] Hayley A Hutchings, Annette Evans, Peter Barnes, Joanne Demmler, Martin Heaven, Melanie A Hyatt, Michelle James-Ellison, Ronan A Lyons, Alison Maddocks, Shantini Paranjothy, et al. Do children who move home and school frequently have poorer educational outcomes in their early years at school? an anonymised cohort study. PloS one, 8(8):e70601, 2013.

[69] Ann John, Joanne McGregor, David Fone, Frank Dunstan, Rosie Cornish, Ronan A Lyons, and Keith R Lloyd. Case-finding for common mental disorders of anxiety and depression in primary care: an external validation of routinely collected data. BMC medical informatics and decision making, 16(1):35, 2016.

[70] A John, AL Marchant, DL Fone, JI McGregor, MS Dennis, JOA Tan, and K Lloyd. Recent trends in primary-care antidepressant prescribing to children and young people: an e-cohort study. Psychological medicine, 46(16):3315-3327, 2016.

[71] Ann John, Michael Dennis, L Kosnes, David Gunnell, J Scourfield, DV Ford, and K Lloyd. Suicide information database-cymru: a protocol for a population-based, 
routinely collected data linkage study to explore risks and patterns of healthcare contact prior to suicide to identify opportunities for intervention. BMJ open, 4 (11):e006780, 2014.

[72] Bethan Bowden, Ann John, Laszlo Trefan, Jennifer Morgan, Daniel Farewell, and David Fone. Risk of suicide following an alcohol-related emergency hospital admission: An electronic cohort study of 2.8 million people. PloS one, 13(4): e0194772, 2018.

[73] David V Ford, Kerina H Jones, Rod M Middleton, Hazel Lockhart-Jones, Inocencio DC Maramba, Gareth J Noble, Lisa A Osborne, and Ronan A Lyons. The feasibility of collecting information from people with multiple sclerosis for the uk ms register via a web portal: characterising a cohort of people with ms. BMC medical informatics and decision making, 12(1):73, 2012.

[74] Kerina H Jones, David V Ford, Philip A Jones, Ann John, Rodden M Middleton, Hazel Lockhart-Jones, Lisa A Osborne, and J Gareth Noble. A large-scale study of anxiety and depression in people with multiple sclerosis: a survey via the web portal of the uk ms register. PLoS One, 7(7):e41910, 2012.

[75] Kerina H Jones, Philip A Jones, Rodden M Middleton, David V Ford, Katie Tuite-Dalton, Hazel Lockhart-Jones, Jeffrey Peng, Ronan A Lyons, Ann John, and J Gareth Noble. Physical disability, anxiety and depression in people with ms: an internet-based survey via the uk ms register. PLoS One, 9(8):e104604, 2014.

[76] Kerina H Jones, David V Ford, Philip A Jones, Ann John, Rodden M Middleton, Hazel Lockhart-Jones, Jeffrey Peng, Lisa A Osborne, and J Gareth Noble. How people with multiple sclerosis rate their quality of life: an eq-5d survey via the uk ms register. PLoS One, 8(6):e65640, 2013.

[77] Kelly Morgan, Mohammed Rahman, Mark Atkinson, Shang-Ming Zhou, Rebecca Hill, Ashrafunnesa Khanom, Shantini Paranjothy, and Sinead Brophy. Association of diabetes in pregnancy with child weight at birth, age 12 months and 5 years-a population-based electronic cohort study. PloS one, 8(11):e79803, 2013.

[78] Adrian Sayers, Daniel Thayer, John N Harvey, Stephen Luzio, Mark D Atkinson, Robert French, Justin T Warner, Colin M Dayan, Susan F Wong, and John W Gregory. Evidence for a persistent, major excess in all cause admissions to hospital in children with type-1 diabetes: results from a large welsh national matched community cohort study. BMJ open, 5(4):e005644, 2015. 
[79] R Robson, AS Lacey, SD Luzio, Hugo Van Woerden, ML Heaven, M Wani, JPJ Halcox, L Castilla-Guerra, J Dawson, and Jonathan Hewitt. Hba1c measurement and relationship to incident stroke. Diabetic Medicine, 33(4):459-462, 2016.

[80] Kymberley Thorne, John G Williams, Ashley Akbari, and Stephen E Roberts. The impact of social deprivation on mortality following acute myocardial infarction, stroke or subarachnoid haemorrhage: A record linkage study. $B M C$ cardiovascular disorders, 15(1):71, 2015.

[81] Majd B Protty, Arron Lacey, Jamie Hayes, and Phillip Freeman. Statins for secondary prevention: clinical use in patients with acute coronary syndrome in wales. Future cardiology, 13(2):137-141, 2017.

[82] Majd Protty, Phillip Freeman, Omar Aldalati, Arron Lacey, William King, Richard Anderson, and Dave Smith. Severe symptomatic aortic stenosis: Medical treatment versus transcatheter aortic valve implantation: A real world analysis of admission profiles, cost, and mortality using the secure anonymised information linkage (sail) databank. Journal of the American College of Cardiology, 65(10 Supplement):A1867, 2015.

[83] William King, Arron Lacey, James White, Daniel Farewell, Frank Dunstan, and David Fone. Equity in healthcare for coronary heart disease, wales (uk) 2004-2010: A population-based electronic cohort study. PloS one, 12(3): e0172618, 2017.

[84] William Owen Pickrell, Arron S Lacey, Rhys H Thomas, Philip EM Smith, and Mark I Rees. Weight change associated with antiepileptic drugs. Journal of Neurology, Neurosurgery $\&$ Psychiatry, pages jnnp-2012, 2012.

[85] Hayley C Gorton, Roger T Webb, Matthew J Carr, Marcos DelPozo-Banos, Ann John, and Darren M Ashcroft. Risk of unnatural mortality in people with epilepsy. JAMA neurology, 2018.

[86] Procedures as a representation of data in a computer program for understanding natural language.

[87] Wendy G Lehnert. The process of question answering. Technical report, DTIC Document, 1977.

[88] Richard Cullingford. Sam. In Readings in natural language processing, pages 627-649. Morgan Kaufmann Publishers Inc., 1986.

[89] Lalit R Bahl, Peter F Brown, Peter V de Souza, and Robert L Mercer. A 
tree-based statistical language model for natural language speech recognition. In Readings in Speech Recognition, pages 507-514. Elsevier, 1990.

[90] Henry Kučera and Winthrop Nelson Francis. Computational analysis of present-day American English. Dartmouth Publishing Group, 1967.

[91] John M Sinclair. Looking up: An account of the COBUILD project in lexical computing and the development of the Collins COBUILD English language dictionary. Collins Elt, 1987.

[92] Barbara B Greene and Gerald M Rubin. Automated grammatical tagging of english. 1971.

[93] Stig Johansson. The lob corpus of british english texts: presentation and comments. ALLC journal, 1(1):25-36, 1980.

[94] Kenneth Ward Church. A stochastic parts program and noun phrase parser for unrestricted text. In Proceedings of the second conference on Applied natural language processing, pages 136-143. Association for Computational Linguistics, 1988.

[95] Steven J DeRose. Grammatical category disambiguation by statistical optimization. Computational linguistics, 14(1):31-39, 1988.

[96] Julian Kupiec. Robust part-of-speech tagging using a hidden markov model. Computer Speech \& Language, 6(3):225-242, 1992.

[97] Eric Brill. A simple rule-based part of speech tagger. In Proceedings of the third conference on Applied natural language processing, pages 152-155. Association for Computational Linguistics, 1992.

[98] Mitchell P Marcus, Mary Ann Marcinkiewicz, and Beatrice Santorini. Building a large annotated corpus of english: The penn treebank. Computational linguistics, 19(2):313-330, 1993.

[99] Stefano Federici, Simonetta Montemagni, and Vito Pirrelli. Shallow parsing and text chunking: a view on underspecification in syntax. Cognitive science research paper-university of Sussex CSRP, pages 35-44, 1996.

[100] Lance A Ramshaw and Mitchell P Marcus. Text chunking using transformation-based learning. In Natural language processing using very large corpora, pages 157-176. Springer, 1999.

[101] Walter Daelemans, Sabine Buchholz, and Jorn Veenstra. Memory-based shallow parsing. arXiv preprint cs/9906005, 1999. 
[102] Tong Zhang, Fred Damerau, and David Johnson. Text chunking using regularized winnow. In Proceedings of the 39th Annual Meeting on Association for Computational Linguistics, pages 539-546. Association for Computational Linguistics, 2001.

[103] Dayne Freitag and Andrew McCallum. Information extraction with hmm structures learned by stochastic optimization. AAAI/IAAI, 2000:584-589, 2000.

[104] Daniel M Bikel, Richard Schwartz, and Ralph M Weischedel. An algorithm that learns what's in a name. Machine learning, 34(1-3):211-231, 1999.

[105] John Lafferty, Andrew McCallum, and Fernando CN Pereira. Conditional random fields: Probabilistic models for segmenting and labeling sequence data. 2001.

[106] Alan R Aronson. Effective mapping of biomedical text to the umls metathesaurus: the metamap program. In Proceedings of the AMIA Symposium, page 17. American Medical Informatics Association, 2001.

[107] Qinghua Zou, Wesley W Chu, Craig Morioka, Gregory H Leazer, and Hooshang Kangarloo. Indexfinder: a method of extracting key concepts from clinical texts for indexing. In AMIA Annual Symposium Proceedings, volume 2003, page 763. American Medical Informatics Association, 2003.

[108] Ralph Grishman and Beth Sundheim. Message understanding conference-6: A brief history. In COLING 1996 Volume 1: The 16th International Conference on Computational Linguistics, volume 1, 1996.

[109] Mark A Przybocki, Jonathan G Fiscus, John S Garofolo, and David S Pallett. 1998 hub-4 information extraction evaluation. In Proc. DARPA Broadcast News Workshop,(Herndon, Va, USA), pages 13-18, 1999.

[110] Satoshi Sekine, Kiyoshi Sudo, and Chikashi Nobata. Extended named entity hierarchy. In $L R E C, 2002$.

[111] George R Doddington, Alexis Mitchell, Mark A Przybocki, Lance A Ramshaw, Stephanie Strassel, and Ralph M Weischedel. The automatic content extraction (ace) program-tasks, data, and evaluation. In $L R E C$, volume 2, page 1, 2004.

[112] Christopher Manning, Mihai Surdeanu, John Bauer, Jenny Finkel, Steven Bethard, and David McClosky. The stanford corenlp natural language processing toolkit. In Proceedings of 52nd annual meeting of the association for computational linguistics: system demonstrations, pages 55-60, 2014. 
[113] Steven Bird and Edward Loper. Nltk: the natural language toolkit. In Proceedings of the ACL 2004 on Interactive poster and demonstration sessions, page 31. Association for Computational Linguistics, 2004.

[114] David Ferrucci and Adam Lally. Uima: an architectural approach to unstructured information processing in the corporate research environment. Natural Language Engineering, 10(3-4):327-348, 2004.

[115] Apache OpenNLP. Apache software foundation. URL http://opennlp. apache. org, 2011.

[116] Guergana K Savova, James J Masanz, Philip V Ogren, Jiaping Zheng, Sunghwan Sohn, Karin C Kipper-Schuler, and Christopher G Chute. Mayo clinical text analysis and knowledge extraction system (ctakes): architecture, component evaluation and applications. Journal of the American Medical Informatics Association, 17(5):507-513, 2010.

[117] Hamish Cunningham. Gate, a general architecture for text engineering. Computers and the Humanities, 36(2):223-254, 2002.

[118] Wei-Dong Jackie Zhu, Bob Foyle, Daniel Gagné, Vijay Gupta, Josemina Magdalen, Amarjeet S Mundi, Tetsuya Nasukawa, Mark Paulis, Jane Singer, Martin Triska, et al. IBM Watson Content Analytics: Discovering Actionable Insight from Your Content. IBM Redbooks, 2014.

[119] Matthew Honnibal and Mark Johnson. An improved non-monotonic transition system for dependency parsing. In Proceedings of the 2015 Conference on Empirical Methods in Natural Language Processing, pages 1373-1378, Lisbon, Portugal, September 2015. Association for Computational Linguistics. URL https://aclweb.org/anthology/D/D15/D15-1162.

[120] I Feinerer and K Hornik. opennlp: opennlp interface. $R$ package version 0.0-8, 2010.

[121] David Meyer, Kurt Hornik, and Ingo Feinerer. Text mining infrastructure in r. Journal of statistical software, 25(5):1-54, 2008.

[122] Peter Kluegl, Martin Toepfer, Philip-Daniel Beck, Georg Fette, and Frank Puppe. Uima ruta: Rapid development of rule-based information extraction applications. Natural Language Engineering, 22(1):1-40, 2016.

[123] R Rehurek and P Sojka. Gensim-python framework for vector space modelling. NLP Centre, Faculty of Informatics, Masaryk University, Brno, Czech Republic, $3(2), 2011$. 
[124] Tomas Mikolov, Ilya Sutskever, Kai Chen, Greg S Corrado, and Jeff Dean. Distributed representations of words and phrases and their compositionality. In Advances in neural information processing systems, pages 3111-3119, 2013.

[125] Jeffrey Pennington, Richard Socher, and Christopher Manning. Glove: Global vectors for word representation. In Proceedings of the 2014 conference on empirical methods in natural language processing (EMNLP), pages 1532-1543, 2014.

[126] James W Perry, Allen Kent, and Madeline M Berry. Machine literature searching x. machine language; factors underlying its design and development. Journal of the Association for Information Science and Technology, 6(4):242-254, 1955.

[127] Jacob Cohen. A coefficient of agreement for nominal scales. Educational and psychological measurement, 20(1):37-46, 1960.

[128] Pontus Stenetorp, Sampo Pyysalo, Goran Topić, Tomoko Ohta, Sophia Ananiadou, and Jun'ichi Tsujii. Brat: a web-based tool for nlp-assisted text annotation. In Proceedings of the Demonstrations at the 13th Conference of the European Chapter of the Association for Computational Linguistics, pages 102-107. Association for Computational Linguistics, 2012.

[129] Brett R South, Shuying Shen, Jianwei Leng, Tyler B Forbush, Scott L DuVall, and Wendy W Chapman. A prototype tool set to support machine-assisted annotation. In Proceedings of the 2012 Workshop on Biomedical Natural Language Processing, pages 130-139. Association for Computational Linguistics, 2012.

[130] Naomi Sager, Carol Friedman, and Margaret S Lyman. Medical language processing: computer management of narrative data. Addison-Wesley Longman Publishing Co., Inc., 1987.

[131] Naomi Sager, Margaret Lyman, Ngo Thanh Nhan, and Leo J Tick. Medical language processing: applications to patient data representation and automatic encoding. Methods of information in medicine, 34(1-2):140-146, 1995.

[132] Lynette Hirschman, Naomi Sager, and Margaret Lyman. Automatic application of health care criteria to narrative patient records. In Proceedings of the Annual Symposium on Computer Application in Medical Care, page 105. American Medical Informatics Association, 1979.

[133] Carol Friedman, Philip O Alderson, John HM Austin, James J Cimino, and Stephen B Johnson. A general natural-language text processor for clinical 
radiology. Journal of the American Medical Informatics Association, 1(2): 161-174, 1994.

[134] Carol Friedman, George Hripcsak, William DuMouchel, Stephen B Johnson, and Paul D Clayton. Natural language processing in an operational clinical information system. Natural Language Engineering, 1(1):83-108, 1995.

[135] Nilesh L Jain and Carol Friedman. Identification of findings suspicious for breast cancer based on natural language processing of mammogram reports. In Proceedings of the AMIA Annual Fall Symposium, page 829. American Medical Informatics Association, 1997.

[136] Carol Friedman, Pauline Kra, Hong Yu, Michael Krauthammer, and Andrey Rzhetsky. Genies: a natural-language processing system for the extraction of molecular pathways from journal articles. In ISMB (supplement of bioinformatics), pages 74-82, 2001.

[137] Marcelo Fiszman, Wendy W Chapman, Dominik Aronsky, R Scott Evans, and Peter J Haug. Automatic detection of acute bacterial pneumonia from chest x-ray reports. Journal of the American Medical Informatics Association, 7(6): 593-604, 2000.

[138] Peter Haug, Spence Koehler, Lee Min Lau, Ping Wang, Roberto Rocha, and Stan Huff. A natural language understanding system combining syntactic and semantic techniques. In Proceedings of the Annual Symposium on Computer Application in Medical Care, page 247. American Medical Informatics Association, 1994.

[139] Katherine P Liao, Tianxi Cai, Guergana K Savova, Shawn N Murphy, Elizabeth W Karlson, Ashwin N Ananthakrishnan, Vivian S Gainer, Stanley Y Shaw, Zongqi Xia, Peter Szolovits, et al. Development of phenotype algorithms using electronic medical records and incorporating natural language processing. bmj, 350:h1885, 2015.

[140] Vincent Damotte and Pierre-Antoine Gourraud. Electronic medical records in multiple sclerosis research. Clinical and Experimental Neuroimmunology, 9(1): $13-18,2018$.

[141] Robert Bill, Serguei Pakhomov, Elizabeth S Chen, Tamara J Winden, Elizabeth W Carter, and Genevieve B Melton. Automated extraction of family history information from clinical notes. In AMIA Annual Symposium Proceedings, volume 2014, page 1709. American Medical Informatics Association, 2014. 
[142] Lifeng Chen and Carol Friedman. Extracting phenotypic information from the literature via natural language processing. In Medinfo, pages 758-762, 2004.

[143] Licong Cui, Alireza Bozorgi, Samden D Lhatoo, Guo-Qiang Zhang, and Satya S Sahoo. Epidea: extracting structured epilepsy and seizure information from patient discharge summaries for cohort identification. In AMIA Annual Symposium Proceedings, volume 2012, page 1191. American Medical Informatics Association, 2012.

[144] Licong Cui, Satya S Sahoo, Samden D Lhatoo, Gaurav Garg, Prashant Rai, Alireza Bozorgi, and Guo-Qiang Zhang. Complex epilepsy phenotype extraction from narrative clinical discharge summaries. Journal of biomedical informatics, 51:272-279, 2014.

[145] Ryan Sullivan, Robert Yao, Randa Jarrar, Jeffrey Buchhalter, and Graciela Gonzalez. Text classification towards detecting misdiagnosis of an epilepsy syndrome in a pediatric population. In AMIA Annual Symposium Proceedings, volume 2014, page 1082. American Medical Informatics Association, 2014.

[146] George Karystianis, Therese Sheppard, William G Dixon, and Goran Nenadic. Modelling and extraction of variability in free-text medication prescriptions from an anonymised primary care electronic medical record research database. BMC medical informatics and decision making, 16(1):18, 2015.

[147] Stuart McTaggart, Clifford Nangle, Jacqueline Caldwell, Samantha Alvarez-Madrazo, Helen Colhoun, and Marion Bennie. Use of text-mining methods to improve efficiency in the calculation of drug exposure to support pharmacoepidemiology studies. International journal of epidemiology, 47(2): 617-624, 2018.

[148] Peter L Elkin, James J Cimino, Henry J Lowe, David B Aronow, Tom H Payne, Pierre S Pincetl, and G Octo Barnett. Mapping to mesh: The art of trapping mesh equivalence from within narrative text. In Proceedings of the annual symposium on computer application in medical care, page 185. American Medical Informatics Association, 1988.

[149] Adam Wilcox, George Hripcsak, and Carol Friedman. Using knowledge sources to improve classification of medical text reports. In Proceedings of Workshop on Text Mining KDD-2000, 2000.

[150] Randolph A Miller, Filip M Gieszczykiewicz, John K Vries, and Gregory F Cooper. Chartline: providing bibliographic references relevant to patient charts using the umls metathesaurus knowledge sources. In Proceedings of the Annual 
Symposium on Computer Application in Medical Care, page 86. American Medical Informatics Association, 1992.

[151] William R Hersh, David H Hickam, R Brian Haynes, and K Ann McKibbon. A performance and failure analysis of saphire with a medline test collection. Journal of the American Medical Informatics Association, 1(1):51-60, 1994.

[152] Prakash Nadkarni, Roland Chen, and Cynthia Brandt. Umls concept indexing for production databases: a feasibility study. Journal of the American Medical Informatics Association, 8(1):80-91, 2001.

[153] Michele Cargill, David Altshuler, James Ireland, Pamela Sklar, Kristin Ardlie, Nila Patil, Charles R Lane, Esther P Lim, Nilesh Kalyanaraman, James Nemesh, et al. Characterization of single-nucleotide polymorphisms in coding regions of human genes. Nature genetics, 22(3):231, 1999.

[154] Francis S Collins, Lisa D Brooks, and Aravinda Chakravarti. A dna polymorphism discovery resource for research on human genetic variation. Genome research, 8(12):1229-1231, 1998.

[155] Wen-Hsiung Li and Lori A Sadler. Low nucleotide diversity in man. Genetics, 129(2):513-523, 1991.

[156] 1000 Genomes Project Consortium et al. A map of human genome variation from population-scale sequencing. Nature, 467(7319):1061-1073, 2010.

[157] Kelly A Frazer, Sarah S Murray, Nicholas J Schork, and Eric J Topol. Human genetic variation and its contribution to complex traits. Nature Reviews Genetics, 10(4):241-251, 2009.

[158] Gabor Marth, Raymond Yeh, Matthew Minton, Rachel Donaldson, Qun Li, Shenghui Duan, Ruth Davenport, Raymond D Miller, and Pui-Yan Kwok. Single-nucleotide polymorphisms in the public domain: how useful are they? Nature genetics, 27(4):371, 2001.

[159] Elizabeth Pennisi. Encode project writes eulogy for junk dna, 2012.

[160] Michael N Weedon, Inês Cebola, Ann-Marie Patch, Sarah E Flanagan, Elisa De Franco, Richard Caswell, Santiago A Rodríguez-Seguí, Charles Shaw-Smith, Candy HH Cho, Hana Lango Allen, et al. Recessive mutations in a distal ptfla enhancer cause isolated pancreatic agenesis. Nature genetics, 46(1):61, 2014.

[161] Kaiyu Jiang, Lisha Zhu, Michael J Buck, Yanmin Chen, Bradley Carrier, Tao Liu, and James N Jarvis. Disease-associated single-nucleotide polymorphisms 
from noncoding regions in juvenile idiopathic arthritis are located within or adjacent to functional genomic elements of human neutrophils and $\mathrm{cd} 4+\mathrm{t}$ cells. Arthritis \&3 Rheumatology, 67(7):1966-1977, 2015.

[162] Kyle Kai-How Farh, Alexander Marson, Jiang Zhu, Markus Kleinewietfeld, William J Housley, Samantha Beik, Noam Shoresh, Holly Whitton, Russell JH Ryan, Alexander A Shishkin, et al. Genetic and epigenetic fine mapping of causal autoimmune disease variants. Nature, 518(7539):337, 2015.

[163] Michael Krawczak, Edward V Ball, Iain Fenton, Peter D Stenson, Shaun Abeysinghe, Nick Thomas, and David N Cooper. Human gene mutation database-a biomedical information and research resource. Human mutation, 15 (1):45, 2000 .

[164] C Sue Richards, Sherri Bale, Daniel B Bellissimo, Soma Das, Wayne W Grody, Madhuri R Hegde, Elaine Lyon, and Brian E Ward. Acmg recommendations for standards for interpretation and reporting of sequence variations: Revisions 2007. Genetics in Medicine, 10(4):294, 2008.

[165] Callum J Bell, Darrell L Dinwiddie, Neil A Miller, Shannon L Hateley, Elena E Ganusova, Joann Mudge, Ray J Langley, Lu Zhang, Clarence C Lee, Faye D Schilkey, et al. Carrier testing for severe childhood recessive diseases by next-generation sequencing. Science translational medicine, 3(65):65ra4-65ra4, 2011.

[166] DG MacArthur, TA Manolio, DP Dimmock, HL Rehm, J Shendure, GR Abecasis, DR Adams, RB Altman, SE Antonarakis, EA Ashley, et al. Guidelines for investigating causality of sequence variants in human disease. Nature, 508(7497):469, 2014.

[167] Bat-sheva Kerem, Johanna M Rommens, Janet A Buchanan, Danuta Markiewicz, Tara K Cox, Aravinda Chakravarti, Manuel Buchwald, and Lap-Chee Tsui. Identification of the cystic fibrosis gene: genetic analysis. Science, 245(4922):1073-1080, 1989.

[168] James F Gusella, Nancy S Wexler, P Michael Conneally, Susan L Naylor, Mary Anne Anderson, Rudolph E Tanzi, Paul C Watkins, Kathleen Ottina, Margaret R Wallace, Alan Y Sakaguchi, et al. A polymorphic dna marker genetically linked to huntington's disease. Nature, 306(5940):234, 1983.

[169] Anna Sillén, Jorge Andrade, Lena Lilius, Charlotte Forsell, Karin Axelman, Jacob Odeberg, Bengt Winblad, and Caroline Graff. Expanded high-resolution 
genetic study of 109 swedish families with alzheimer's disease. European Journal of Human Genetics, 16(2):202, 2008.

[170] Sue Richards, Nazneen Aziz, Sherri Bale, David Bick, Soma Das, Julie Gastier-Foster, Wayne W Grody, Madhuri Hegde, Elaine Lyon, Elaine Spector, et al. Standards and guidelines for the interpretation of sequence variants: a joint consensus recommendation of the american college of medical genetics and genomics and the association for molecular pathology. Genetics in medicine, 17 (5):405, 2015.

[171] Elizabeth M Smigielski, Karl Sirotkin, Minghong Ward, and Stephen T Sherry. dbsnp: a database of single nucleotide polymorphisms. Nucleic acids research, 28(1):352-355, 2000.

[172] Frederick Sanger, Steven Nicklen, and Alan R Coulson. Dna sequencing with chain-terminating inhibitors. Proceedings of the national academy of sciences, 74(12):5463-5467, 1977.

[173] Robert D Fleischmann, Mark D Adams, Owen White, Rebecca A Clayton, Ewen F Kirkness, Anthony R Kerlavage, Carol J Bult, Jean-Francois Tomb, Brian A Dougherty, Joseph M Merrick, et al. Whole-genome random sequencing and assembly of haemophilus influenzae rd. Science, 269(5223):496-512, 1995.

[174] André Goffeau, Bart G Barrell, Howard Bussey, RW Davis, Bernard Dujon, Heinz Feldmann, Francis Galibert, JD Hoheisel, Cr Jacq, Michael Johnston, et al. Life with 6000 genes. Science, 274(5287):546-567, 1996.

[175] The C. elegans Sequencing Consortium. Genome sequence of the nematode c. elegans: a platform for investigating biology. Science, pages 2012-2018, 1998.

[176] Mark D Adams, Susan E Celniker, Robert A Holt, Cheryl A Evans, Jeannine D Gocayne, Peter G Amanatides, Steven E Scherer, Peter W Li, Roger A Hoskins, Richard F Galle, et al. The genome sequence of drosophila melanogaster. Science, 287(5461):2185-2195, 2000.

[177] J Craig Venter, Mark D Adams, Eugene W Myers, Peter W Li, Richard J Mural, Granger G Sutton, Hamilton O Smith, Mark Yandell, Cheryl A Evans, Robert A Holt, et al. The sequence of the human genome. science, 291(5507): 1304-1351, 2001.

[178] Nayanah Siva. 1000 genomes project, 2008.

[179] Genomics England. The 100,000 genomes project. The, 100:0-2, 2016. 
[180] Cathie Sudlow, John Gallacher, Naomi Allen, Valerie Beral, Paul Burton, John Danesh, Paul Downey, Paul Elliott, Jane Green, Martin Landray, et al. Uk biobank: an open access resource for identifying the causes of a wide range of complex diseases of middle and old age. PLoS medicine, 12(3):e1001779, 2015.

[181] Konrad J Karczewski, Ben Weisburd, Brett Thomas, Matthew Solomonson, Douglas M Ruderfer, David Kavanagh, Tymor Hamamsy, Monkol Lek, Kaitlin E Samocha, Beryl B Cummings, et al. The exac browser: displaying reference data information from over 60000 exomes. Nucleic acids research, 45(D1): D840-D845, 2016.

[182] Elaine R Mardis. The impact of next-generation sequencing technology on genetics. Trends in genetics, 24(3):133-141, 2008.

[183] David A Wheeler, Maithreyan Srinivasan, Michael Egholm, Yufeng Shen, Lei Chen, Amy McGuire, Wen He, Yi-Ju Chen, Vinod Makhijani, G Thomas Roth, et al. The complete genome of an individual by massively parallel dna sequencing. nature, 452(7189):872, 2008.

[184] Shamil Sunyaev, Vasily Ramensky, and Peer Bork. Towards a structural basis of human non-synonymous single nucleotide polymorphisms. Trends in Genetics, 16(5):198-200, 2000.

[185] Carles Ferrer-Costa, Modesto Orozco, and Xavier de la Cruz. Characterization of disease-associated single amino acid polymorphisms in terms of sequence and structure properties1. Journal of molecular biology, 315(4):771-786, 2002.

[186] Pauline C Ng and Steven Henikoff. Predicting deleterious amino acid substitutions. Genome research, 11(5):863-874, 2001.

[187] Pauline C Ng and Steven Henikoff. Sift: Predicting amino acid changes that affect protein function. Nucleic acids research, 31(13):3812-3814, 2003.

[188] James Ulrich Bowie and Robert T Sauer. Identifying determinants of folding and activity for a protein of unknown structure. Proceedings of the National Academy of Sciences, 86(7):2152-2156, 1989.

[189] Alyssa Bentley, Bridget MacLennan, Jonathan Calvo, and Charles R Dearolf. Targeted recovery of mutations in drosophila. Genetics, 156(3):1169-1173, 2000.

[190] Yijing Chen, Della Yee, Katherine Dains, Aurobindo Chatterjee, James Cavalcoli, Elizabeth Schneider, Jinsop Om, Richard P Woychik, and Terry Magnuson. Genotype-based screen for enu-induced mutations in mouse embryonic stem cells. Nature genetics, 24(3):314, 2000. 
[191] Peter Markiewicz, Lynn G Kleina, Christina Cruz, Susannah Ehret, and Jeffrey H Miller. Genetic studies of the lac repressor. xiv. analysis of 4000 altered escherichia coli lac repressors reveals essential and non-essential residues, as well as" spacers" which do not require a specific sequence. Journal of molecular biology, 240(5):421-433, 1994.

[192] Jörg Suckow, Peter Markiewicz, Lynn G Kleina, Jeffrey Miller, Brigitte Kisters-Woike, and Benno Müller-Hill. Genetic studies of the lac repressor xv: 4000 single amino acid substitutions and analysis of the resulting phenotypes on the basis of the protein structure. Journal of molecular biology, 261(4):509-523, 1996.

[193] Daniel D Loeb, Ronald Swanstrom, Lorraine Everitt, Marianne Manchester, Susan E Stamper, and Clyde A Hutchison III. Complete mutagenesis of the hiv-1 protease. Nature, 340(6232):397, 1989.

[194] Dale Rennell, Suzanne E Bouvier, Larry W Hardy, and Anthony R Poteete. Systematic mutation of bacteriophage t4 lysozyme. Journal of molecular biology, 222(1):67-88, 1991.

[195] Stephen F Altschul, Thomas L Madden, Alejandro A Schäffer, Jinghui Zhang, Zheng Zhang, Webb Miller, and David J Lipman. Gapped blast and psi-blast: a new generation of protein database search programs. Nucleic acids research, 25(17):3389-3402, 1997.

[196] Ivan A Adzhubei, Steffen Schmidt, Leonid Peshkin, Vasily E Ramensky, Anna Gerasimova, Peer Bork, Alexey S Kondrashov, and Shamil R Sunyaev. A method and server for predicting damaging missense mutations. Nature methods, 7(4): $248,2010$.

[197] Shamil R Sunyaev, Frank Eisenhaber, Igor V Rodchenkov, Birgit Eisenhaber, Vladimir G Tumanyan, and Eugene N Kuznetsov. Psic: profile extraction from sequence alignments with position-specific counts of independent observations. Protein engineering, 12(5):387-394, 1999.

[198] Anders Krogh, BjoÈrn Larsson, Gunnar Von Heijne, and Erik LL Sonnhammer. Predicting transmembrane protein topology with a hidden markov model: application to complete genomes1. Journal of molecular biology, 305(3):567-580, 2001.

[199] Andrei Lupas, Marc Van Dyke, and Jeff Stock. Predicting coiled coils from protein sequences. Science, pages 1162-1164, 1991. 
[200] Henrik Nielsen, Jacob Engelbrecht, Søren Brunak, and Gunnar von Heijne. Identification of prokaryotic and eukaryotic signal peptides and prediction of their cleavage sites. Protein engineering, 10(1):1-6, 1997.

[201] Wolfgang Kabsch and Christian Sander. Dictionary of protein secondary structure: pattern recognition of hydrogen-bonded and geometrical features. Biopolymers, 22(12):2577-2637, 1983.

[202] Helen M Berman, Tammy Battistuz, Talapady N Bhat, Wolfgang F Bluhm, Philip E Bourne, Kyle Burkhardt, Zukang Feng, Gary L Gilliland, Lisa Iype, Shri Jain, et al. The protein data bank. Acta Crystallographica Section D: Biological Crystallography, 58(6):899-907, 2002.

[203] Vasily Ramensky, Peer Bork, and Shamil Sunyaev. Human non-synonymous snps: server and survey. Nucleic acids research, 30(17):3894-3900, 2002.

[204] Daniel Quang, Yifei Chen, and Xiaohui Xie. Dann: a deep learning approach for annotating the pathogenicity of genetic variants. Bioinformatics, 31(5): 761-763, 2014.

[205] Chengliang Dong, Peng Wei, Xueqiu Jian, Richard Gibbs, Eric Boerwinkle, Kai Wang, and Xiaoming Liu. Comparison and integration of deleteriousness prediction methods for nonsynonymous snvs in whole exome sequencing studies. Human molecular genetics, 24(8):2125-2137, 2014.

[206] Boris Reva, Yevgeniy Antipin, and Chris Sander. Predicting the functional impact of protein mutations: application to cancer genomics. Nucleic acids research, 39(17):e118-e118, 2011.

[207] Nilah M Ioannidis, Joseph H Rothstein, Vikas Pejaver, Sumit Middha, Shannon K McDonnell, Saurabh Baheti, Anthony Musolf, Qing Li, Emily Holzinger, Danielle Karyadi, et al. Revel: an ensemble method for predicting the pathogenicity of rare missense variants. The American Journal of Human Genetics, 99(4):877-885, 2016.

[208] Hashem A Shihab, Julian Gough, David N Cooper, Peter D Stenson, Gary LA Barker, Keith J Edwards, Ian NM Day, and Tom R Gaunt. Predicting the functional, molecular, and phenotypic consequences of amino acid substitutions using hidden markov models. Human mutation, 34(1):57-65, 2013.

[209] Martin Kircher, Daniela M Witten, Preti Jain, Brian J O'roak, Gregory M Cooper, and Jay Shendure. A general framework for estimating the relative pathogenicity of human genetic variants. Nature genetics, 46(3):310, 2014. 
[210] Hannah Carter, Christopher Douville, Peter D Stenson, David N Cooper, and Rachel Karchin. Identifying mendelian disease genes with the variant effect scoring tool. BMC genomics, 14(3):S3, 2013.

[211] Emidio Capriotti and Piero Fariselli. Phd-snpg: a webserver and lightweight tool for scoring single nucleotide variants. Nucleic acids research, 45(W1): W247-W252, 2017.

[212] Melissa J Landrum, Jennifer M Lee, George R Riley, Wonhee Jang, Wendy S Rubinstein, Deanna M Church, and Donna R Maglott. Clinvar: public archive of relationships among sequence variation and human phenotype. Nucleic acids research, 42(D1):D980-D985, 2013.

[213] D Fredman, Marianne Siegfried, Yan P. Yuan, Peer Bork, Heikki Lehväslaiho, and Anthony J Brookes. Hgvbase: a human sequence variation database emphasizing data quality and a broad spectrum of data sources. Nucleic acids research, 30(1):387-391, 2002.

[214] Ada Hamosh, Alan F Scott, Joanna S Amberger, Carol A Bocchini, and Victor A McKusick. Online mendelian inheritance in man (omim), a knowledgebase of human genes and genetic disorders. Nucleic acids research, 33(suppl 1): D514-D517, 2005.

[215] Kevin G Becker, Kathleen C Barnes, Tiffani J Bright, and S Alex Wang. The genetic association database. Nature genetics, 36(5):431-432, 2004.

[216] David N Cooper, Edward V Ball, and Michael Krawczak. The human gene mutation database. Nucleic acids research, 26(1):285-287, 1998.

[217] Stephen F Altschul, Warren Gish, Webb Miller, Eugene W Myers, and David J Lipman. Basic local alignment search tool. Journal of molecular biology, 215 (3):403-410, 1990.

[218] Robert C Edgar. Muscle: multiple sequence alignment with high accuracy and high throughput. Nucleic acids research, 32(5):1792-1797, 2004.

[219] Julie D Thompson, Desmond G Higgins, and Toby J Gibson. Clustal w: improving the sensitivity of progressive multiple sequence alignment through sequence weighting, position-specific gap penalties and weight matrix choice. Nucleic acids research, 22(22):4673-4680, 1994.

[220] Robert C Edgar. Search and clustering orders of magnitude faster than blast. Bioinformatics, 26(19):2460-2461, 2010. 
[221] Robert D Finn, Jody Clements, and Sean R Eddy. Hmmer web server: interactive sequence similarity searching. Nucleic acids research, 39(suppl_2): W29-W37, 2011.

[222] Katherine S Pollard, Melissa J Hubisz, Kate R Rosenbloom, and Adam Siepel. Detection of nonneutral substitution rates on mammalian phylogenies. Genome research, 20(1):110-121, 2010.

[223] Adam Siepel, Gill Bejerano, Jakob S Pedersen, Angie S Hinrichs, Minmei Hou, Kate Rosenbloom, Hiram Clawson, John Spieth, LaDeana W Hillier, Stephen Richards, et al. Evolutionarily conserved elements in vertebrate, insect, worm, and yeast genomes. Genome research, 15(8):1034-1050, 2005.

[224] Manuel Garber, Mitchell Guttman, Michele Clamp, Michael C Zody, Nir Friedman, and Xiaohui Xie. Identifying novel constrained elements by exploiting biased substitution patterns. Bioinformatics, 25(12):i54-i62, 2009.

[225] Liam J McGuffin, Kevin Bryson, and David T Jones. The psipred protein structure prediction server. Bioinformatics, 16(4):404-405, 2000.

[226] Shide Liang, Chi Zhang, Song Liu, and Yaoqi Zhou. Protein binding site prediction using an empirical scoring function. Nucleic acids research, 34(13): 3698-3707, 2006.

[227] Alexei Vazquez, Alessandro Flammini, Amos Maritan, and Alessandro Vespignani. Global protein function prediction from protein-protein interaction networks. Nature biotechnology, 21(6):697, 2003.

[228] Xiaoming Liu, Xueqiu Jian, and Eric Boerwinkle. dbnsfp: a lightweight database of human nonsynonymous snps and their functional predictions. Human mutation, 32(8):894-899, 2011.

[229] William McLaren, Laurent Gil, Sarah E Hunt, Harpreet Singh Riat, Graham RS Ritchie, Anja Thormann, Paul Flicek, and Fiona Cunningham. The ensembl variant effect predictor. Genome biology, 17(1):122, 2016.

[230] Pablo Cingolani, Adrian Platts, Le Lily Wang, Melissa Coon, Tung Nguyen, Luan Wang, Susan J Land, Xiangyi Lu, and Douglas M Ruden. A program for annotating and predicting the effects of single nucleotide polymorphisms, snpeff: Snps in the genome of drosophila melanogaster strain w1118; iso-2; iso-3. Fly, 6(2):80-92, 2012.

[231] Kai Wang, Mingyao Li, and Hakon Hakonarson. Annovar: functional annotation 
of genetic variants from high-throughput sequencing data. Nucleic acids research, 38(16):e164-e164, 2010.

[232] Henk Harkema, John N Dowling, Tyler Thornblade, and Wendy W Chapman. Context: an algorithm for determining negation, experiencer, and temporal status from clinical reports. Journal of biomedical informatics, 42(5):839-851, 2009.

[233] John Moult, Jan T Pedersen, Richard Judson, and Krzysztof Fidelis. A large-scale experiment to assess protein structure prediction methods. Proteins: Structure, Function, and Bioinformatics, 23(3), 1995.

[234] David T Jones. Protein secondary structure prediction based on position-specific scoring matrices. Journal of molecular biology, 292(2):195-202, 1999.

[235] William J Youden. Index for rating diagnostic tests. Cancer, 3(1):32-35, 1950.

[236] Michael Tan, Ian Wilson, Vanessa Braganza, Sophia Ignatiadis, Ray Boston, Vijaya Sundararajan, Mark J Cook, and Wendyl J D'Souza. Development and validation of an epidemiologic case definition of epilepsy for use with routinely collected australian health data. Epilepsy \& Behavior, 51:65-72, 2015.

[237] C Franchi, G Giussani, P Messina, M Montesano, S Romi, A Nobili, I Fortino, A Bortolotti, L Merlino, E Beghi, et al. Validation of healthcare administrative data for the diagnosis of epilepsy. J Epidemiol Community Health, pages jech-2013, 2013.

[238] E Wayne Holden, Elizabeth Grossman, Hoang Thanh Nguyen, Margaret J Gunter, Becky Grebosky, Ann Von Worley, Leila Nelson, Scott Robinson, and David J Thurman. Developing a computer algorithm to identify epilepsy cases in managed care organizations. Disease Management, 8(1):1-14, 2005.

[239] Wilhelmine Hadler Meeraus, Irene Petersen, Richard Frank Chin, Felicity Knott, and Ruth Gilbert. Childhood epilepsy recorded in primary care in the uk. Archives of disease in childhood, 98(3):195-202, 2013.

[240] Vanessa Delgado Nunes, Laura Sawyer, Julie Neilson, Grammati Sarri, and J Helen Cross. Diagnosis and management of the epilepsies in adults and children: summary of updated nice guidance. Bmj, 344:e281, 2012.

[241] Rebecca Louise Bromley, George E Mawer, Maria Briggs, Christopher Cheyne, Jill Clayton-Smith, Marta García-Fiñana, Rachel Kneen, Sam B Lucas, Rebekah Shallcross, Gus A Baker, et al. The prevalence of neurodevelopmental disorders 
in children prenatally exposed to antiepileptic drugs. J Neurol Neurosurg Psychiatry, 84(6):637-643, 2013.

[242] Karl Titze, Sabine Koch, Hans Helge, Ulrike Lehmkuhl, Hellgard Rauh, and Hans-Christoph Steinhausen. Prenatal and family risks of children born to mothers with epilepsy: effects on cognitive development. Developmental Medicine 6 Child Neurology, 50(2):117-122, 2008.

[243] Kimford J Meador, Gus A Baker, Nancy Browning, Morris J Cohen, Rebecca L Bromley, Jill Clayton-Smith, Laura A Kalayjian, Andres Kanner, Joyce D Liporace, Page B Pennell, et al. Fetal antiepileptic drug exposure and cognitive outcomes at age 6 years (nead study): a prospective observational study. The Lancet Neurology, 12(3):244-252, 2013.

[244] Giouliana Kadra, Robert Stewart, Hitesh Shetty, Richard G Jackson, Mark A Greenwood, Angus Roberts, Chin-Kuo Chang, James H MacCabe, and Richard D Hayes. Extracting antipsychotic polypharmacy data from electronic health records: developing and evaluating a novel process. BMC psychiatry, 15 (1):166, 2015.

[245] Brad Gulko, Ilan Gronau, Melissa J Hubisz, and Adam Siepel. Probabilities of fitness consequences for point mutations across the human genome. bioRxiv, page 006825, 2014.

[246] Qiongshi Lu, Yiming Hu, Jiehuan Sun, Yuwei Cheng, Kei-Hoi Cheung, and Hongyu Zhao. A statistical framework to predict functional non-coding regions in the human genome through integrated analysis of annotation data. Scientific reports, 5:10576, 2015.

[247] Iuliana Ionita-Laza, Kenneth McCallum, Bin Xu, and Joseph D Buxbaum. A spectral approach integrating functional genomic annotations for coding and noncoding variants. Nature genetics, 48(2):214, 2016.

[248] 1000 Genomes Project Consortium et al. An integrated map of genetic variation from 1,092 human genomes. Nature, 491(7422):56, 2012.

[249] Yongwook Choi and Agnes P Chan. Provean web server: a tool to predict the functional effect of amino acid substitutions and indels. Bioinformatics, 31(16): 2745-2747, 2015.

[250] Inna Dubchak, Ilya Muchnik, Christopher Mayor, Igor Dralyuk, and Sung-Hou Kim. Recognition of a protein fold in the context of the scop classification. Proteins: Structure, Function, and Bioinformatics, 35(4):401-407, 1999. 
[251] Jenny Eachus, Mark Williams, Philip Chan, George Davey Smith, Matthew Grainge, Jenny Donovan, and Stephen Frankel. Deprivation and cause specific morbidity: evidence from the somerset and avon survey of health. Bmj, 312 (7026):287-292, 1996.

[252] Walter F Stewart, Richard B Lipton, David D Celentano, and Michael L Reed. Prevalence of migraine headache in the united states: relation to age, income, race, and other sociodemographic factors. Jama, 267(1):64-69, 1992.

[253] Anthony K Ngugi, SM Kariuki, C Bottomley, I Kleinschmidt, JW Sander, and CR Newton. Incidence of epilepsy a systematic review and meta-analysis. Neurology, 77(10):1005-1012, 2011.

[254] Mohammad Reza Mohammadi, Ahmad Ghanizadeh, Haratoun Davidian, Mohammad Mohammadi, and Maryam Norouzian. Prevalence of epilepsy and comorbidity of psychiatric disorders in iran. Seizure-European Journal of Epilepsy, 15(7):476-482, 2006.

[255] Morris J Cohen, Kimford J Meador, Nancy Browning, Gus A Baker, Jill Clayton-Smith, Laura A Kalayjian, Andres Kanner, Joyce D Liporace, Page B Pennell, Michael Privitera, et al. Fetal antiepileptic drug exposure: motor, adaptive, and emotional/behavioral functioning at age 3 years. Epilepsy $\mathscr{6}$ Behavior, 22(2):240-246, 2011.

[256] Rebecca L Bromley, George Mawer, Jenna Love, James Kelly, Laura Purdy, Lauren McEwan, Maria Briggs, Jill Clayton Smith, Xin Sin, and Gus A Baker. Early cognitive development in children born to women with epilepsy: a prospective report. Epilepsia, 51(10):2058-2065, 2010.

[257] Kimford J Meador, Gus A Baker, Nancy Browning, Morris J Cohen, Jill Clayton-Smith, Laura A Kalayjian, Andres Kanner, Joyce D Liporace, Page B Pennell, Michael Privitera, et al. Foetal antiepileptic drug exposure and verbal versus non-verbal abilities at three years of age. Brain, 134(2):396-404, 2011.

[258] Rebecca L Bromley, Rebecca Calderbank, Christopher P Cheyne, Claire Rooney, Penny Trayner, Jill Clayton-Smith, Marta García-Fiñana, Beth Irwin, James Irvine Morrow, Rebekah Shallcross, et al. Cognition in school-age children exposed to levetiracetam, topiramate, or sodium valproate. Neurology, 87(18): 1943-1953, 2016.

[259] Eija Gaily, Elisa Kantola-Sorsa, Vilho Hiilesmaa, M Isoaho, Riitta Matila, Mervi Kotila, T Nylund, A Bardy, E Kaaja, and M-L Granström. Normal 
intelligence in children with prenatal exposure to carbamazepine. Neurology, 62 (1):28-32, 2004.

[260] Yanshan Wang, Liwei Wang, Majid Rastegar-Mojarad, Sungrim Moon, Feichen Shen, Naveed Afzal, Sijia Liu, Yuqun Zeng, Saeed Mehrabi, Sunghwan Sohn, et al. Clinical information extraction applications: A literature review. Journal of biomedical informatics, 2017.

[261] Honghan Wu, Giulia Toti, Katherine I Morley, Zina Ibrahim, Amos Folarin, Ismail Kartoglu, Richard Jackson, Asha Agrawal, Clive Stringer, Darren Gale, et al. Semehr: surfacing semantic data from clinical notes in electronic health records for tailored care, trial recruitment, and clinical research. The Lancet, 390:S97, 2017.

[262] Ray R Larson. Introduction to information retrieval. Journal of the American Society for Information Science and Technology, 61(4):852-853, 2010.

[263] Deepak Ravichandran, Patrick Pantel, and Eduard Hovy. Randomized algorithms and nlp: using locality sensitive hash function for high speed noun clustering. In Proceedings of the 43rd annual meeting on association for computational linguistics, pages 622-629. Association for Computational Linguistics, 2005.

[264] Lauren C Walters-Sen, Sayaka Hashimoto, Devon Lamb Thrush, Shalini Reshmi, Julie M Gastier-Foster, Caroline Astbury, and Robert E Pyatt. Variability in pathogenicity prediction programs: impact on clinical diagnostics. Molecular genetics $\&$ genomic medicine, 3(2):99-110, 2015.

[265] Lisa A Miosge, Matthew A Field, Yovina Sontani, Vicky Cho, Simon Johnson, Anna Palkova, Bhavani Balakishnan, Rong Liang, Yafei Zhang, Stephen Lyon, et al. Comparison of predicted and actual consequences of missense mutations. Proceedings of the National Academy of Sciences, 112(37):E5189-E5198, 2015.

[266] Janita Thusberg, Ayodeji Olatubosun, and Mauno Vihinen. Performance of mutation pathogenicity prediction methods on missense variants. Human mutation, 32(4):358-368, 2011.

[267] Emidio Capriotti and Russ B Altman. Improving the prediction of disease-related variants using protein three-dimensional structure. $B M C$ bioinformatics, 12(Suppl 4):S3, 2011.

[268] Christoph Lossin. A catalog of scn1a variants. Brain and Development, 31(2): 114-130, 2009. 
[269] Margarida C Lopes, Chris Joyce, Graham RS Ritchie, Sally L John, Fiona Cunningham, Jennifer Asimit, and Eleftheria Zeggini. A combined functional annotation score for non-synonymous variants. Human heredity, 73(1):47-51, 2012. 